\title{
CHEMICAL, PHYSICAL, AND RADIOLOGICAL QUALITY OF SELECTED PUBLIC WATER SUPPLIES IN FLORIDA, NOVEMBER 1977-FEBRUARY 1978
}

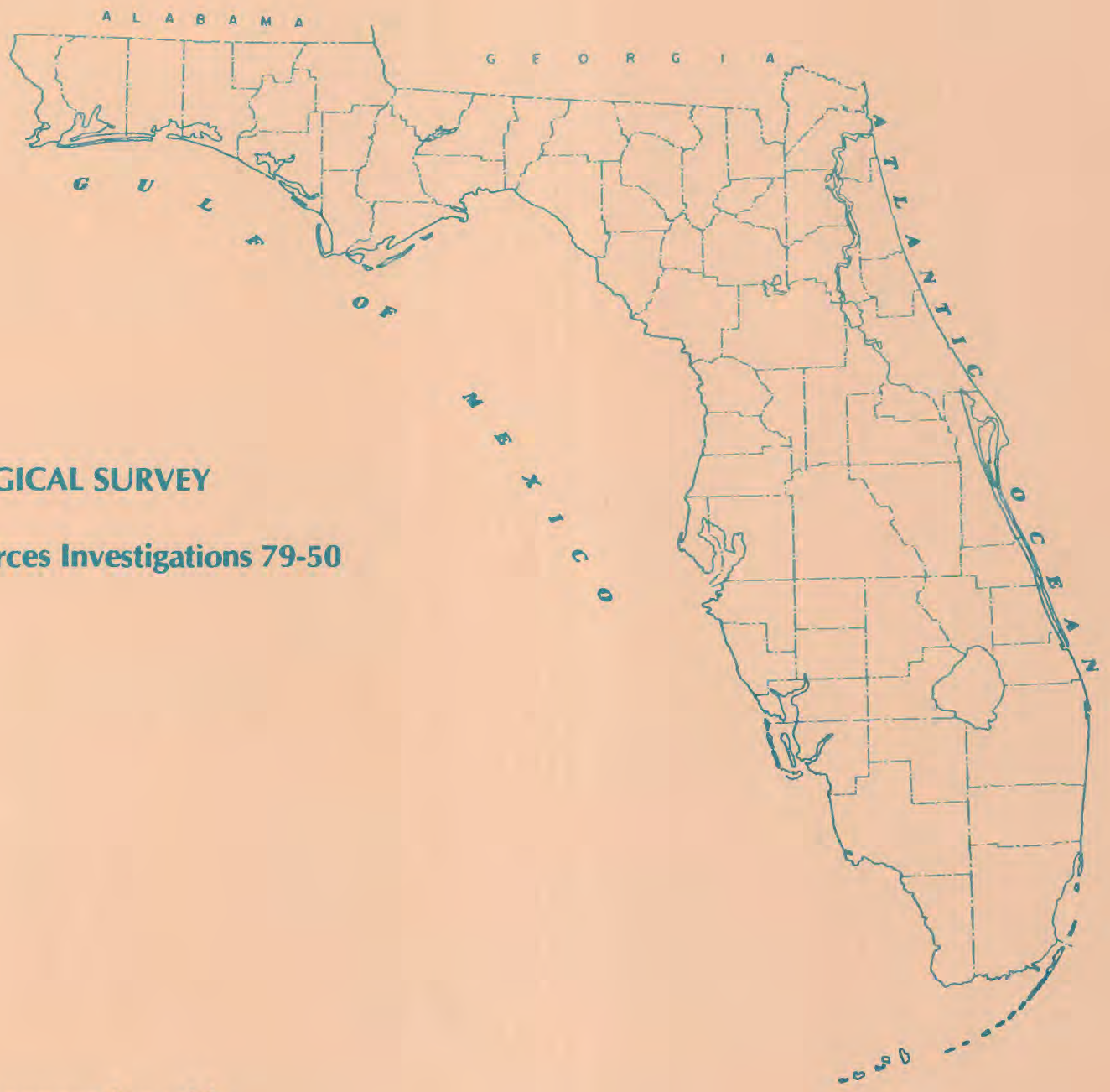

Prepared in cooperation with FLORIDA DEPARTMENT OF ENVIRONMENTAL REGULATION BUREAU OF DRINKING WATER AND SPECIAL PROGRAMS 


\begin{tabular}{|c|c|}
\hline $\begin{array}{l}\text { BIBLIOGRAPHIC DATA } \\
\text { SHEET }\end{array}$ & 3. Recipient's Accession No. \\
\hline \multirow{2}{*}{$\begin{array}{l}\text { 4. Title and Subtitle } \\
\text { CHEMICAL, PHYSICAL, AND RADIOLOGICAL QUALITY OF SELECTED PUBLIC } \\
\text { WATER SUPPLIES IN FLORIDA, NOVEMBER 1977-FEBRUARY } 1978\end{array}$} & $\begin{array}{l}\text { 5. Report Date } \\
\text { April } 1979\end{array}$ \\
\hline & 6. \\
\hline $\begin{array}{l}\text { 7. Author(s) } \\
\text { George A. Irwin and Robert W. Hull }\end{array}$ & $\begin{array}{l}\text { 8. Performing Organization Rept. } \\
{ }^{N} \text { USGS/WRI 79-50 }\end{array}$ \\
\hline 9. Performing Organization Name and Address & 10. Project/Task/Work Unit No. \\
\hline $\begin{array}{l}\text { U.S. Geological Survey, Water Resources Division } \\
325 \text { John Knox Road } \\
\text { Suite F-240 } \\
\text { Tallahassee, Florida } 32303\end{array}$ & 11. Contract/Grant No. \\
\hline $\begin{array}{l}\text { 12. Sponsoring Organization Name and Address } \\
\text { U.S. Geological Survey, Water Resources Division } \\
325 \text { John Knox Road }\end{array}$ & $\begin{array}{l}\text { 13. Type of Report \& Period } \\
\text { Covered }\end{array}$ \\
\hline $\begin{array}{l}\text { Suite F-240 } \\
\text { Tallahassee, Florida } 32303\end{array}$ & 14. \\
\hline
\end{tabular}

15. Supplementary Notes

Prepared in cooperation with Florida Department of Environmental Regulation, Bureau of Drinking Water and Special Programs.

16. Abstracts

Virtually all treated public water supplies sampled in Florida meet the National Inter Primary and Proposed Secondary Drinking Water Regulations. These findings are based o a water-quality reconnaissance of 129 treated public supplies throughout the State during the period November 1977 through February 1978. While primary drinking water regulation exceedences were infrequent, lead, selenium, and gross alpha radioactivity in a very few water supplies were above established maximum contaminant levels. Additionally, the secondary drinking water regulation parameters--dissolved solids, chloride, sulfate, iron, color, and pH--were occasionally detected in excess of the proposed Federal regulations. The secondary regulations, however, pertain nainly to t aesthetic quality of drinking water and not directly to public health aspects.

17. Key Words and Document Analysis. 17a. Descriptors

Potable (drinking) water, water quality, desalination, water treatment, public utilities, water supply, surface water, ground water

17b. Identifiers/Open-Ended Terms

Florida, Floridan aquifer, Biscayne aquifer, sand and gravel aquifer, shallow sand aquifer

17c. COSATI Field/Group

18. Availability Statement

No restriction on distribution.

\begin{tabular}{|l|c|}
\hline $\begin{array}{l}\text { 19. Security Class (This } \\
\text { Report) } \\
\text { UNCLASSIFIED }\end{array}$ & $\begin{array}{c}\text { 21. No. of Pages } \\
174\end{array}$ \\
\hline $\begin{array}{l}\text { 20. Security Class (This } \\
\text { Page } \\
\text { UNCLASSIFIED }\end{array}$ & 22. Price \\
\hline
\end{tabular}


CHEMICAL, PHYSICAL, AND RADIOLOGICAL QUALITY OF SELECTED

PUBLIC WATER SUPPLIES IN FLORIDA, NOVEMBER 1977-FEBRUARY 1978

By G. A. Irwin and R. W. Hu11

U.S. GEOLOGICAL SURVEY

Water-Resources Investigations 79-50

Prepared in cooperation with

FLORIDA DEPARTMENT OF ENVIRONMENTAL REGULATION BUREAU OF DRINKING WATER AND SPECIAL PROGRAMS 
UNITED STATES DEPARTMENT OF THE INTERIOR

CECIL D. ANDRUS, Secretary

GEOLOGICAL SURVEY

H. William Menard, Director

For additional information write to:

U.S. Geological Survey

325 John Knox Road

Suite $F-240$

Tallahassee, Florida 32303 


\section{ILLUSTRATIONS}

Page

Figure 1. Map showing location of public water supply sampling sites in northwestern Florida- 13

2. Map showing location of public water supply sampling sites in north and northeastern Florida--_--- 14

3. Map showing location of public water supply sampling sites in central and central-coastal Florida------ 15

4. Map showing location of public water supply sampling

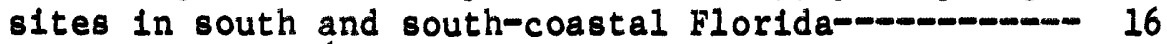

5. Graphs of distribution of concentration ranges of Nat1onal Interim Primary Drinking Water Regulation parameters for selected treated public water supplles---- 30

6. Graphs of distribution of concentration ranges of Proposed National Secondary Drinking Water Regulation parameters for selected treated public water supplies--

\section{TABLES}

Table 1. Summary of procedures for water-sample treatment and methods of analysis for selected chemical constituents and physical properties-- 6

2. Location of public water supplies sampled from November 1977 through February 1978-

3. Summary of chemical and physical analyses of National Interim Primary Drinking Water Regulation parameters for selected treated surface-water supplies--------

4. Summary of chemical and physical analyses of National Interim Primary Drinking Water Regulation parameters for selected untreated surface-water supplies-------

5. Summary of chemical and physical analyses of Proposed National Secondary Drinking Water Regulation parameters for selected treated surface-water supplies--

6. Summary of chemical and physical analyses of Proposed National Secondary Drinking Water Regulation parameters for selected untreated surface-water supplies

7. Summary of chemical and physical analyses of National Interim Primary Drinking Water Regulation parameters for selected treated public ground-water supplies---

8. Summary of chemical and physical analyses of Proposed National Secondary Drinking Water Regulation parameters for selected treated public ground-water supplies---_- 
CHEMICAL, PHYSICAL, AND RADIOLOGICAL QUALITY OF SELECTED

PUBLIC WATER SUPPLIES IN FLORIDA, NOVEMBER 1977-FEBRUARY 1978

By G. A. Irwin and R. W. Hul1

ABSTRACT

Virtually all treated public water supplies sampled in Florida meet the National Interim Primary and Proposed Secondary Drinking Water Regulations. These findings are based on a water-quality reconnalssance of 129 treated public supplies throughout the State during the perlod November 1977 through February 1978.

While primary drinking water regulation exceedences were infrequent, lead, selenium, and gross alpha radioactivity in a very few water supplies were above established maximum contaminant levels. Add1tionally, the secondary drinking water regulation parameters-dissolved solids, chloride, sulfate, 1ron, color, and pH-were occasionally detected in excess of the proposed Federal regulations. The secondary regulations, however, pertain mainly to the aesthetic quality of drinking water and not directly to public health aspects.

\section{INTRODUCTION}

On December 16, 1974, Publ1c Law 93-523, commonly referred to as the "Safe Drinking Water Act" was adopted by the U.S. Congress and was signed into law by President Gerald R. Ford. As a result of this legislation, the U.S. Environmental Protection Agency on March 14, 1975, released in the Federal Register, the "National Interim Primary Drinking Water Regulations." A revision of the 1nterim regulations was published in the Federal Register on December 24, 1975. Promulgation of the interim regulations was for 18 months subsequent to publication in December 1975. On June 24, 1977, the Nationa1 Interim Primary Drinking Water Regulations went into effect. These regulations supersede, but closely parallel, the drinking water standards as set forth in 1962 by the U.S. Public Health Service.

A supplementary Federal Register issuance to the primary standards was released on July 9, 1976. These regulations added maximum contaminant levels for selected natural and man-made radioactivity. The effective date for the radioactivity regulations, June 24, 1977, was set to coincide with the initial primary regulations.

On March 31, 1977, the Environmental Protect1on Agency released in the Federal Register the proposed National Secondary Drinking Water Regulations. The secondary regulations were set forth to complement the primary regulations. They are, however, not Federally enforceable, 
but are intended as guidelines for state policy. The secondary regulations mainly pertain to aesthetic qualities of drinking water.

The overall objective of the Safe Drinking Water Act is to encourage public water-supply agencies to provide water of high quality to users throughout the United States by establishing and malntaining national standards. This goal is to be achieved primarily by expanding the scope and level of water utility service, thus improving the quality and dependability of the nation's drinking water.

Public Law 93-523 is a Federal Act; however, primacy under the Act becomes the responsibility of individual states upon their request to, and approval by, the U.S. Environmental Protection Agency. The State of Florida, having assumed primacy, designated the Department of Environmental Regulation, Bureau of Drinking Water and Special Programs, as the agency responsible for coordinating all public drinking water supply activities in the State. In July 1976, the Department and the Geological Survey entered into a cooperative hydrologic investigation to evaluate the water quality of selected public supplies in Florida. As an inftial endeavor during August-September 1976, both the untreated (raw) and treated (after processing by treatment plant) waters from 127 public supplies were sampled throughout the State. The sampling emphasis was on the chemical variables designated in the National Interim Primary Drinking Water Regulations. The reconnaissance had two objectives. The first objective was to determine the quality of the treated water distributed to users by selected public supply agencies. The second objective was to evaluate the quality of untreated or source waters used by those agencies. The concentration and distribution of some of the chemical constituents determined during this reconnaissance were virtually unknown in many parts of Florida. One hundred and twenty seven treated and untreated public water supplies were sampled during the 1976 reconnaissance and the results are presented in Irwin and Healy, 1978.

During November 1977-February 1978 a second sampling reconnaissance was conducted. The scope of the reconnalssance for 1977-78 was essentially the same as for the 1976 sampling, except for a significant increase in the water quality parameter coverage. Additional analytical coverage for 1977-78 included secondary drinking water regulation parameters at all sites and gross radioactivity at selected sites.

\section{DESCRIPTION OF THE SAMPLING PROGRAM}

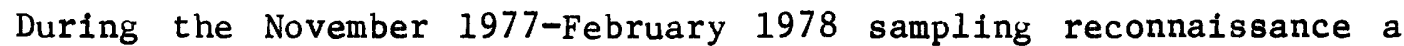
total of 129 public water supplies were sampled. Specifically, 116 public water supplies were sampled for primary and secondary drinking water regulation parameters, some excluding gross radioactivity. Gross radioactivity was determined for 90 public water supplies. A few supplies which were sampled in 1976 were sampled just for radioactivity 
in 1977-78. Additional chemical analyses, such as major inorganic constituents and physical properties were performed on most of the supplies. A complete presentation of the 1977-78 analytical results are given beginning on page 41 .

The chemical constituents of principal interest to the objectives of this study were those that have elther established (primary regulations) or proposed (secondary regulations) maximum contaminant levels (MCL). A list of constituents and their respective maximum contaminant levels are as follows:

\section{National Primary Drinking Water Regulations}

\begin{tabular}{cc}
\hline Constituent & $\begin{array}{c}\text { Maximum contaminant } \\
\text { level in milligrams } \\
\text { per 1iter } 19\end{array}$ \\
\hline
\end{tabular}

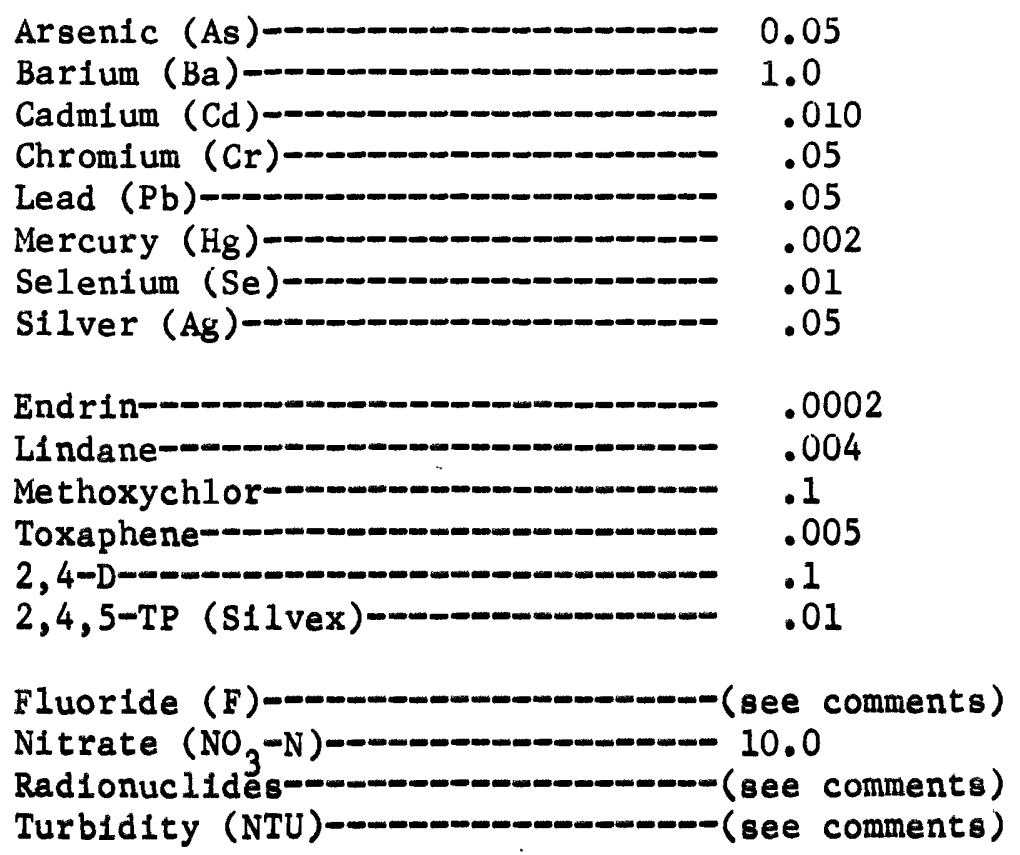

Fluoride: When the annual average daily air temperatures for the location in which the water supply system is located is the following, the maximum contaminant level for fluoride is:

1/ Milligrams per liter (mg/L) may be converted to microyrams per liter (ug/L) by multiplying by 1,000 . 


\begin{tabular}{ccc}
$\begin{array}{c}\text { Temperature, } \\
\text { in degrees } \\
\text { Fahrenheit }\end{array}$ & $\begin{array}{c}\text { Temperature, } \\
\text { in degrees } \\
\text { Celsius }\end{array}$ & $\begin{array}{c}\text { Maximum contaminant } \\
\text { level in milligrams } \\
\text { per liter }\end{array}$ \\
\hline 53.7 and below & 12.0 and below & 2.4 \\
53.8 to 58.3 & 12.1 to 14.6 & 2.2 \\
58.4 to 63.8 & 14.7 to 17.6 & 2.0 \\
63.9 to 70.6 & 17.7 to 21.4 & 1.8 \\
70.7 to 79.2 & 21.5 to 26.2 & 1.6 \\
79.3 to 90.5 & 26.3 to 32.5 & 1.4 \\
\hline
\end{tabular}

When adjusting fluoride concentrations with the addition of fluoridecontaining compounds, State Health Department recommendations should be followed.

Radionuclides: The following are the maximum contaminant levels for radium-226, radium-228, gross alpha particle radioactivity, beta particle, and photon radioactivity:

a. Combined radium-226 and radium-228; $5 \mathrm{pCl} / \mathrm{L}$ (picocuries per liter).

b. Gross alpha particle activity (including radium-226, but excluding radon and uranium); $15 \mathrm{pCi} / \mathrm{L}$.

c. The average annual concentration of beta particle and photon radioactivity from man-made radionuclides in drinking water shall not produce an annual dose equivalent to the total body or any internal organ greater than 4 millirem/year (rem, roentgen equivalent man).

Turbidity: The maximum contaminant level is a monthly average of 1 nephelometric turbidity unit (NTU) or 5 turbidity units (NTU) with State approval, provided it does not interfere with disinfection, maintenance of chlorine residual, or bacteriological testing.

Proposed Secondary Drinking Water Regulations

\begin{tabular}{|c|c|}
\hline Constituent & $\begin{array}{l}\text { Maximum contaminant } \\
\text { level in milligrams } \\
\text { per liter, except as } \\
\text { indicated }\end{array}$ \\
\hline Chloride (C & $---\infty$ \\
\hline Color (Pt-C & $-\infty-\infty$ \\
\hline Copper $(\mathrm{Cu})$ & 1.0 \\
\hline Corrosivity & -- Non-corrosive \\
\hline Foaming age & $-\infty$ \\
\hline Hydrogen su. & ---- \\
\hline Iron $(\mathrm{Fe})-$ & $-\infty-\infty$ \\
\hline Manganese ( & $-\infty-\infty$ \\
\hline $\begin{array}{l}\text { Odor- } \\
\text { pH (units)- }\end{array}$ & $\begin{array}{ll}\text { threshold odor number } \\
6.5-8.5\end{array}$ \\
\hline Sulfate (SC & $-\quad 250$ \\
\hline Total diss & 500 \\
\hline Zinc $(Z n)-$ & 5.0 \\
\hline
\end{tabular}


The methods used in analyzing water for the parameters cited in this report, except as footnoted, are described in Brown, Skougstad, and Fishman (1970) and Goerlitz and Brown (1972) and Fishman and Brown (1976). Equivalency between the U.S. Geological Survey and the U.S. Environmenta1 Protection Agency methods for Public Law 92-500 is documented in section $304(\mathrm{~g})$ amended, Pub1ic Law 92-500, Federal Register, December 1, 1976. A summary of analytical methods and procedures for sample treatment for each chemical constituent and physical property is given in table 1. Specifically regarding Public Law 93-523, the Geological Survey also considers these methods to be equivalent to the methods prescribed by the Environmental Protection Agency for determining compliance with the maximum contaminant levels of the "Safe Drinking Water Act."

\section{Procedures for Sample Treatment and Analytical Services}

When required, preanalysis treatment of the water sample was performed in the field at the time of collection. As soon as possible after the samples were collected and treated they were transported either to the U.S. Geological Survey Central Water Quality Laboratory in Doraville, Ga., or to the U.S. Geological Survey Radiochemical Laboratory in Arvada, Colo., for subsequent analysis.

Specific conductance was generally determined both in the field and in the laboratory. The specific conductance value determined in the field was reported preferentially to that measured in the laboratory. Water temperature and $\mathrm{pH}$ measurements were made in the field at the time of sampling.

\section{LOCATION OF SELECTED PUBLIC WATER SUPPLY SAMPLING SITES}

The location of the public water supplies sampled during November 1977 through February 1978 are given in table 2. In addition to site location by county and city (usua1ly), table 2 also gives a figure number reference by which each sampling site may be located on one of the four maps presented in this section (figs. 1-4).

\section{RESULTS \\ Public Surface-Water Supplies}

During the November 1977 to February 1978 reconnaissance most of the principal public surface-water supplies in the State (table 2 and figures 1-4) were analyzed for those parameters included in the primary and secondary drinking water regulations. Analyses were also made for additional selected major inorganic chemical and physical parameters. A minimum of one sample (treated water) was collected and analyzed for each of the supplies and for most supplies an analysis was performed on both the raw and treated water. A list of the surface-water public supplies are given in table 2. A complete data presentation for each public supply is given beginning on page 41 . 


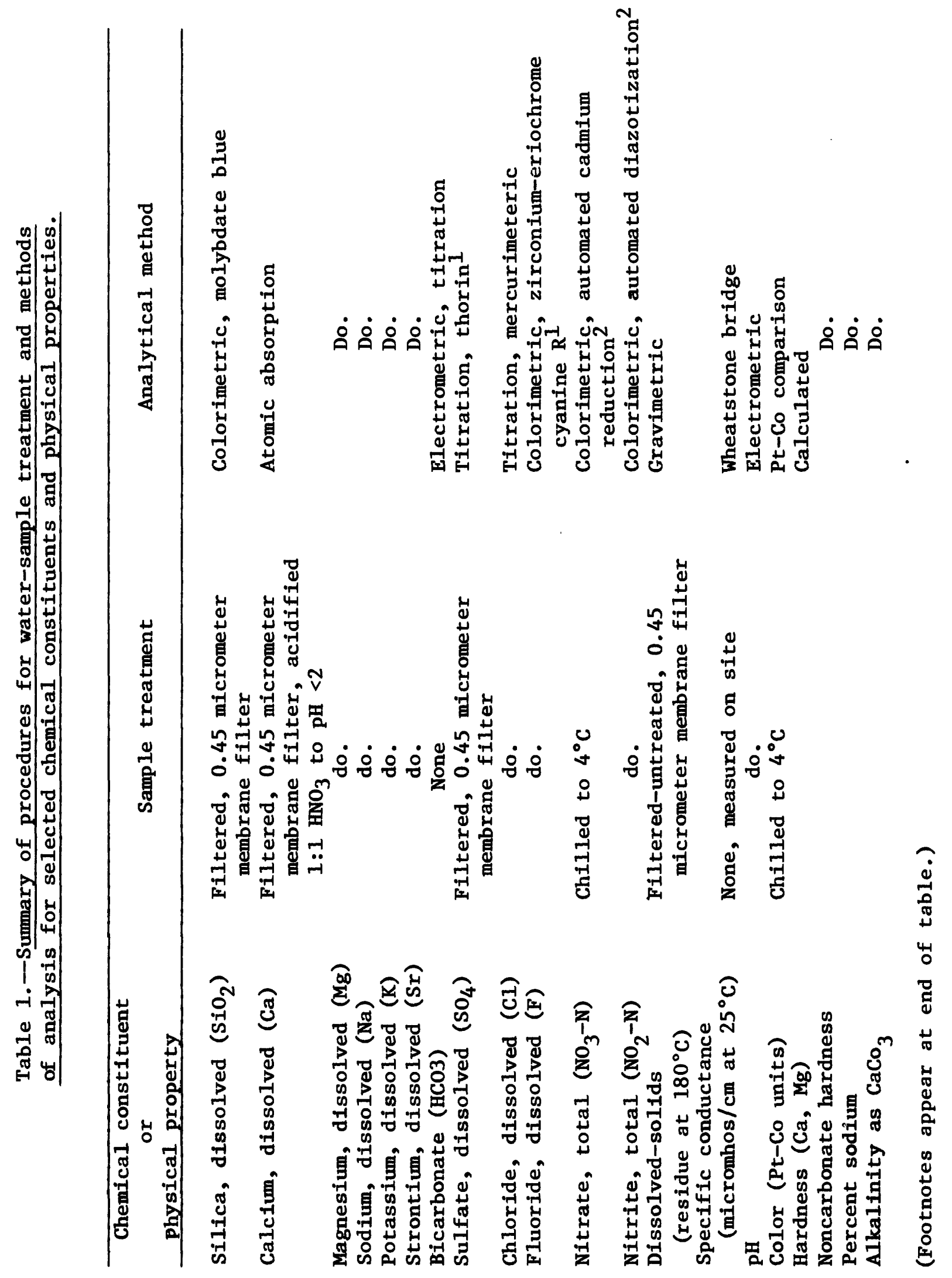




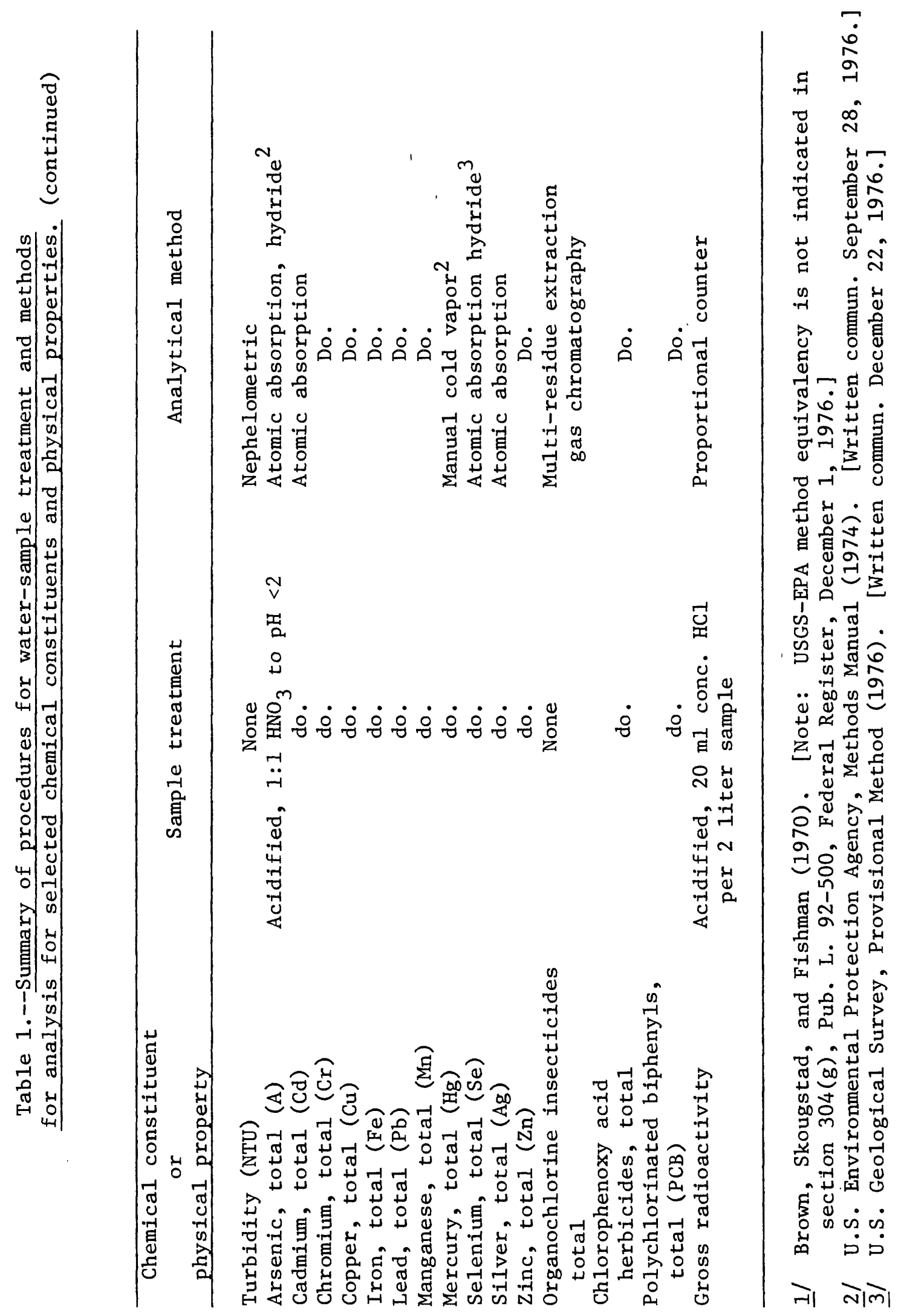


Table 2.--Location of Public Water Supplies sampled from November 1977 through February 1978.

County

Site

Figure

number

Bay

Bay County Water System (surface water) 1

Lynn Haven

Mexico Beach

1

1

Brevard

Melbourne (surface water)

3

Mims

3

Broward

Coll1er Estates

Davie

Pembroke Pines

Sunrise

Tamarac

4

4

4

4

4

Charlotte

Port Charlotte (surface water)

Punta Gorda (surface water)

Rotonda West

4

4

4

Citrus

Crystal River

3

Clay

Orange Park (Meadowbrook)

2

Collier

Immokalee

Marco Island (surface water)

4

4

Columbia

Lake C1ty

2

Dade

Florida C1ty

North Miami

Rex Utilities (Florida City)

South Miami Heights

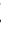

Arcadia

G. Pierce Wood Memorial Hospital

De Soto

Atlantic Beach

2

Jacksonville

Jacksonville Beach

Jacksonville Suburban Utility

Neptune Beach

2

2

2

Flagler

Bunne11

3

Flagler Beach

3

Palm Coast 
Table 2.--Location of Public Water Supplies sampled from November 1977 through February 1978. (continued)

County

Franklin

Gadsden

Gulf

Hamilton

Hardee

Hendry

Hernando

Highlands

Hi11sborough

Indian River

Jackson

Lake

Lee

Leon
Site

Apalachicola

Chattahoochee (Florida State Hospital)

(surface water)

Havana

Quincy (surface water)

Port St. Joe (surface water)

Wewahitchka

Jennings

White Springs

Zolfo Springs

Clewiston (surface water)

Figure

number

2

2

4

4

Ridge Manor

3

Weeki Wachee

3

Avon Park

4

Carrolwood

3

Ruskin

Tampa (surface water)

3

Temple Terrace

3

3

Gifford

3

Graceville

Sneads

1

1

Clermont

3

Eustis

3

Groveland

3

Mt. Dora

3

Umatilla

3

Lee County Water System (surface water) 4

Woodville 
Table 2.--Location of Public Water Supplies sampled from November 1977 through February 1978. (continued)

County

Levy

Madison

Manatee

Marion

Martin

Nassau

Okaloosa

Okeechobee

Orange

Osceola

Palm Beach

Pasco
Chiefland

Williston

Site

Figure

number

Greenville $\quad 2$

Bradenton (surface water)

4

Manatee County Water System

(surface water)

4

Belleview

Hobe Sound

Indiantown

4

4

Callahan

Fernandina Beach

Hilliard

2

2

2

Auburn 1

Baker

1

Okeechobee (surface water) 4

Conway (First Florida Utilities) 3

Walt Disney World 3

St. Cloud 3

Belle Glade (surface water) 4

Century Village Utilities 4

First Florida Utilities 4

Manalapan

Pahokee (surface water)

Palm Beach -Villa Del Ray

West Palm Beach (surface water) 4

Colonial Hills 3

Delmar Corporation 3

Forest Hills 3

Holiday Lake Estates 3

Hudson Community Water Works 3

Port Richey 3

San Antonio 3

Zephyrhills 3 
Table 2.--Location of Public Water Supplies sampled from November 1977 through February 1978. (contInued)

County

Site

F1gure number

Pinellas

Bellair

Polk

Fort Meade

Lake Alfred

Mulberry

Wahneta

Ridge Utilities

Eaton Park

Garden Grove

Imperial Lakes

Jan Phyl Village

Frostproof

Putnam

Crescent City

2

Interlachen

St. Johns

Anastasia Sanitary District

2

Sawgrass

St. Augustine (surface water)

St. Lucle

North Port St. Lucie

Santa Rosa

Navarre Beach

1

Pace

1

Sarasota

Englewood

North Port Charlotte (surface water)

Sarasota

Venice

Ventce Gardens

\section{4}

4

4

4

4

Seminole

Indian Hills

3

Longwood

Sumter

Wildwood

3

Suwannee

Branford

2

Taylor

Keaton Beach

2

Union

Union Correctional Institute 
Table 2.--Location of Public Water Supplies sampled from November 1977 through February 1978. (continued)

County

Volusia

Waku1la

Walton

Washington
Site

Holly Hill

Deltona

New Smyrna Beach

Panacea

St. Marks

South Walton County Utilities

Paxton

Vernon
Figure

number

3

3

3

2

2

1

1

1 


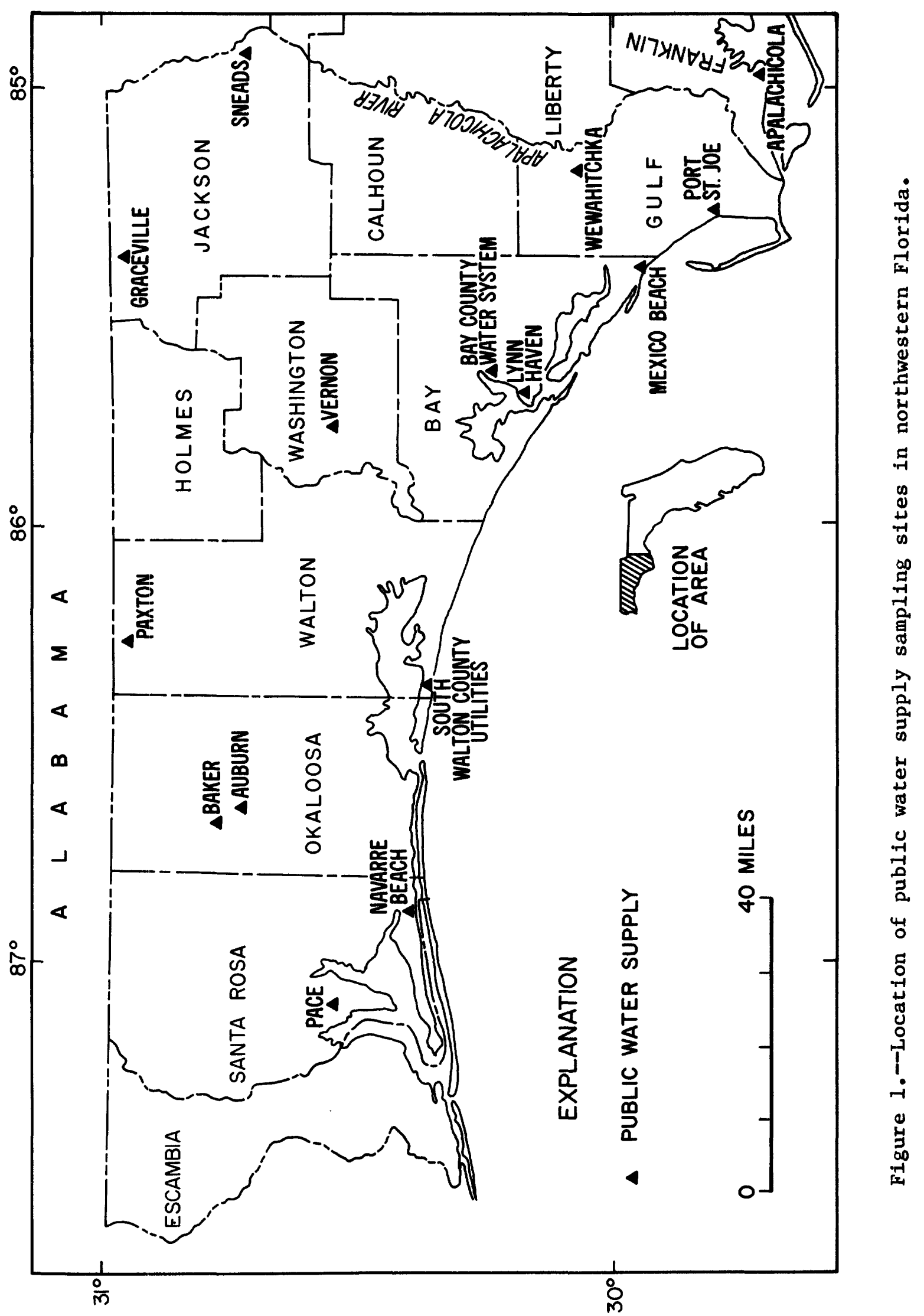




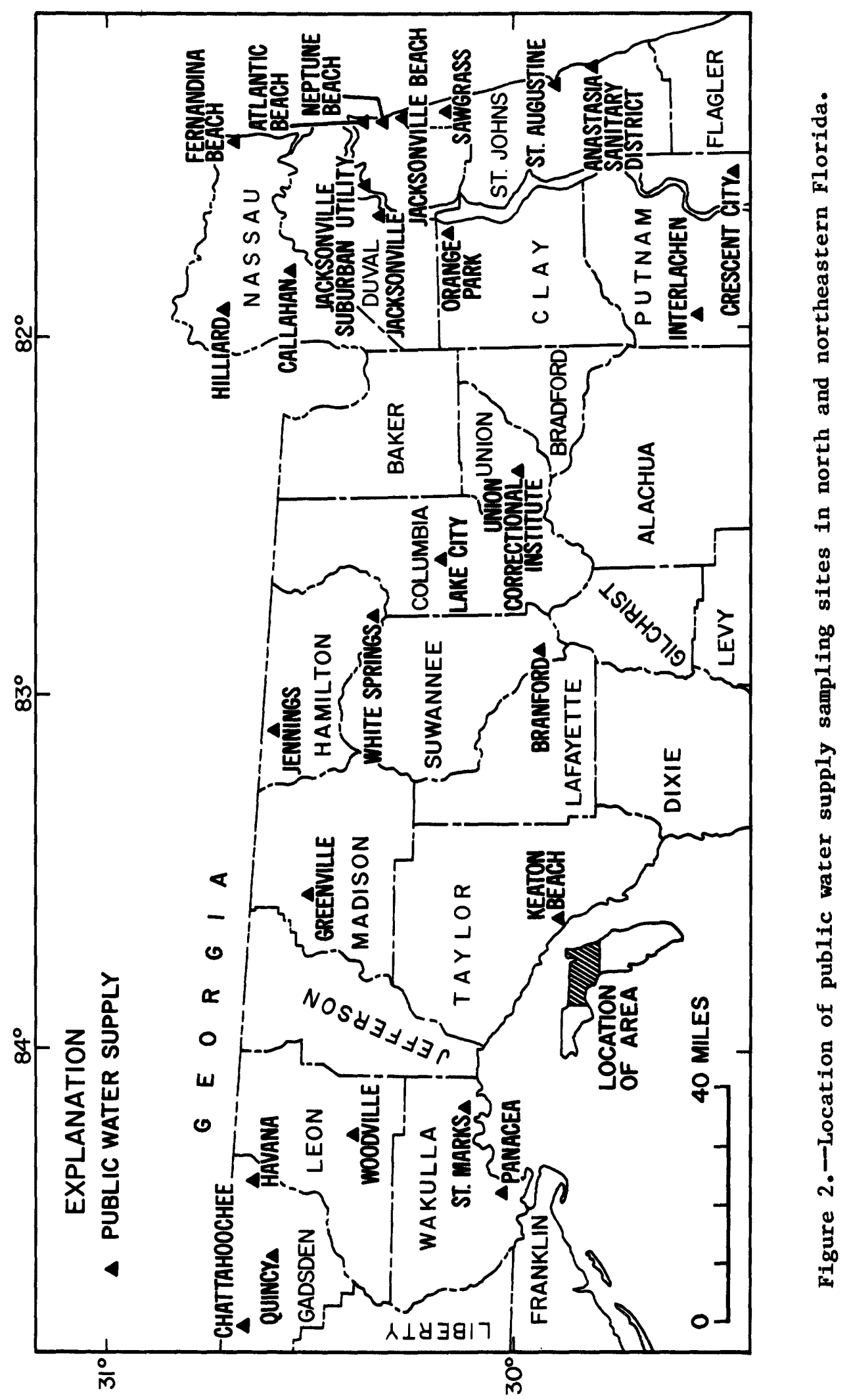




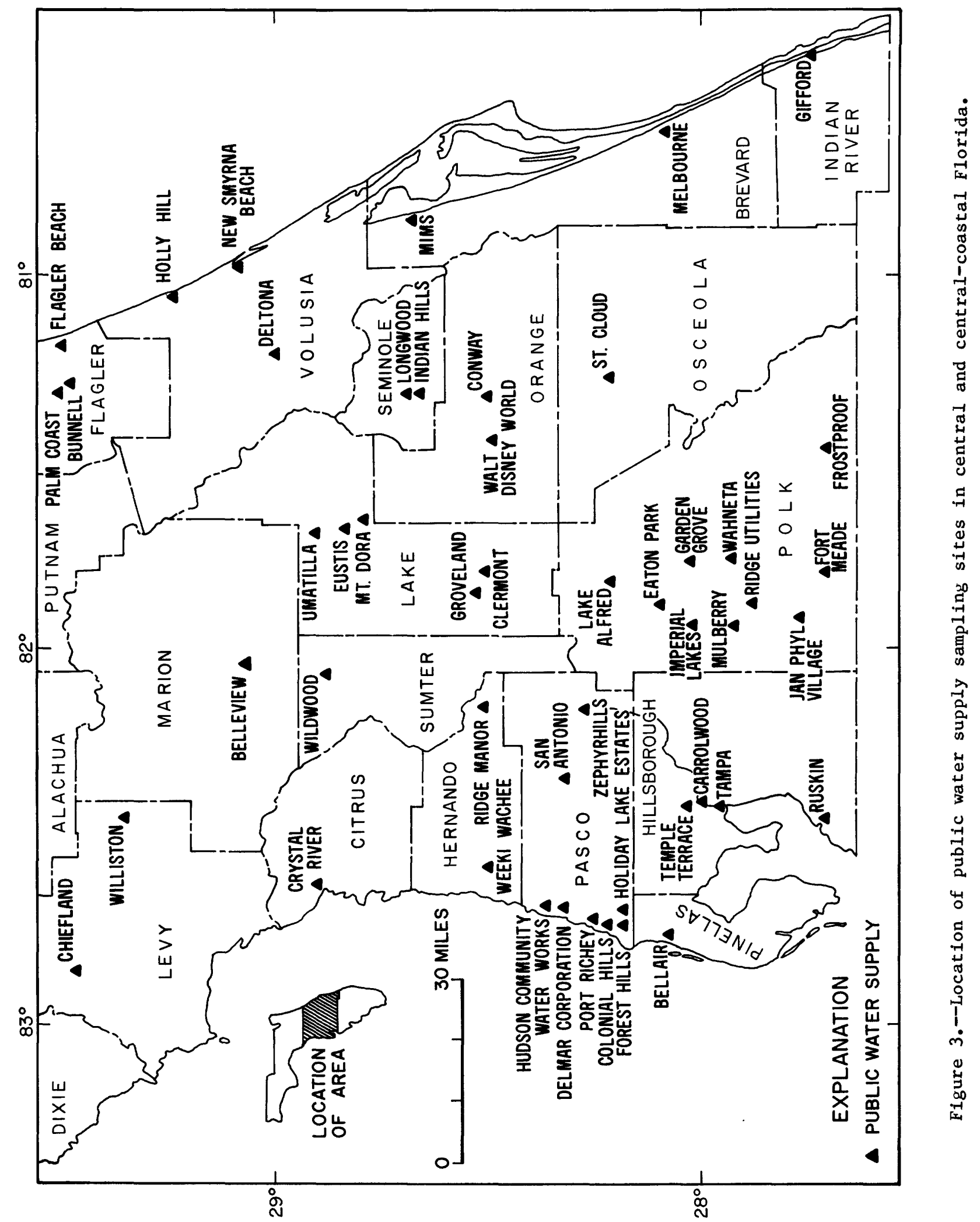




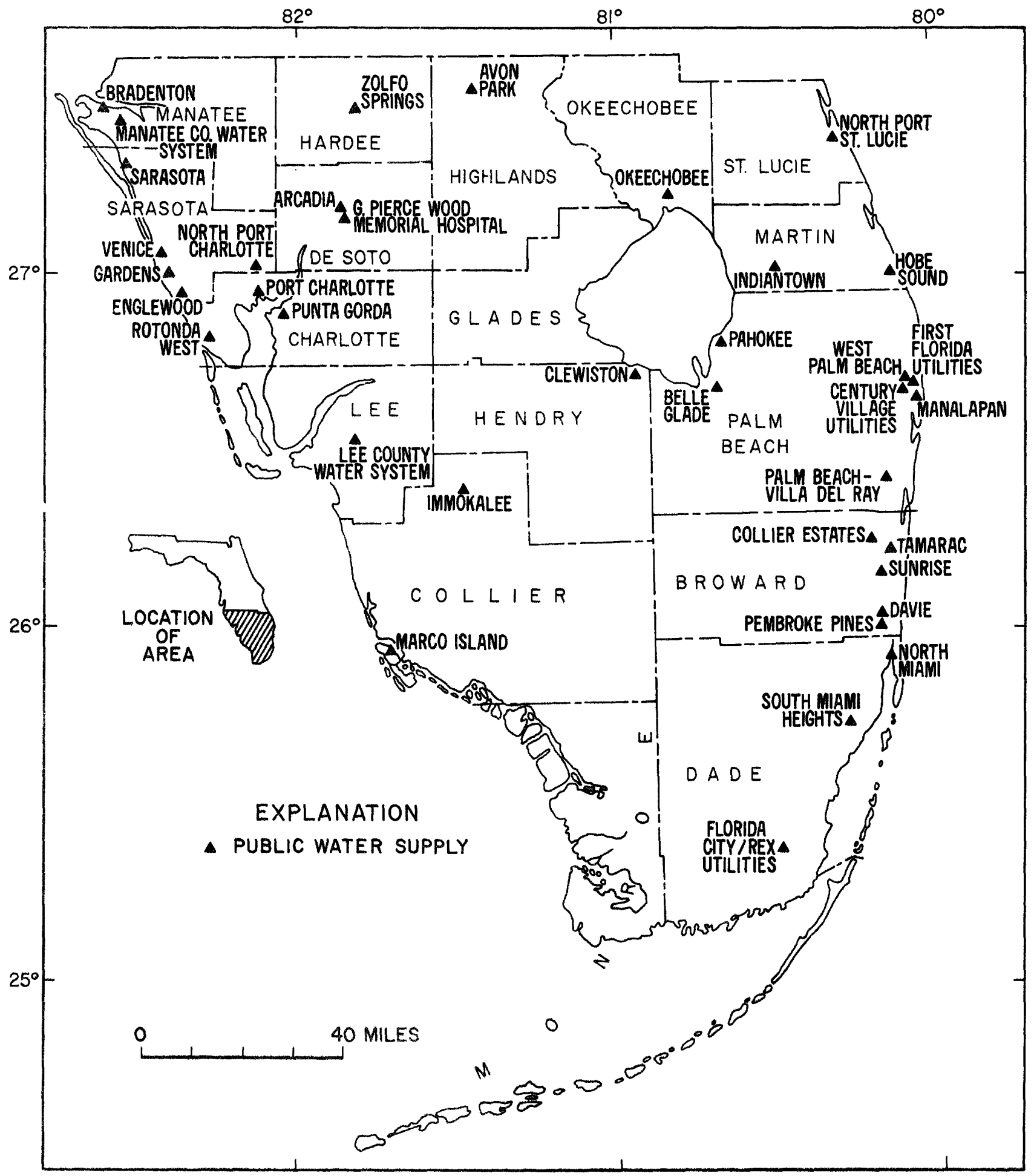

Figure 4.--Location of public water supply sampling sites in south and south-coastal Florida. 
On the average the major inorganic chemical composition of the raw surface water is cation-anion mixed. Calcium is dominant comprising about 49 percent of the cations with bicarbonate and chloride comprising 42 and 36 percent, respectively, of the anions. The concentration of calcium averaged $47 \mathrm{mg} / \mathrm{L}$ and ranged from $4.0 \mathrm{mg} / \mathrm{L}$ in Quincy Creek, Gadsden County, to $170 \mathrm{mg} / \mathrm{L}$ in the surface water from the infiltration gallery near St. Augustine in St. Johns County. Bicarbonate averaged $122 \mathrm{mg} / \mathrm{L}$ and chloride averaged $61 \mathrm{mg} / \mathrm{L}$. The minimum bicarbonate and chloride concentrations were $19 \mathrm{mg} / \mathrm{L}$ and $5.1 \mathrm{mg} / \mathrm{L}$ occurring in Quincy Creek. The maximum bicarbonate was $390 \mathrm{mg} / \mathrm{L}$ in the St. Augustine water supply and the maximum chloride was $150 \mathrm{mg} / \mathrm{L}$ occurring in the surfacewater supplies for West Palm Beach (Clear Lake) and Belle Glade (Lake Okeechobee) both in Palm Beach County.

The average dissolved solids concentration of the raw surface water supplies was $306 \mathrm{mg} / \mathrm{L}$; however, a rather large variance existed as the standard deviation was $181 \mathrm{mg} / \mathrm{L}$. The dissolved solids range was $36 \mathrm{mg} / \mathrm{L}$ (Quincy Creek) to $632 \mathrm{mg} / \mathrm{L}$ (Belle Glade). The water hardness averaged $160 \mathrm{mg} / \mathrm{L}$ with a standard deviation of $110 \mathrm{mg} / \mathrm{L}$ and ranged from $16 \mathrm{mg} / \mathrm{L}$ (Quincy Creek) to $440 \mathrm{mg} / \mathrm{L}$ (St. Augustine).

The treated surface-water was also a mixed cation-anion composition with calcium-sodium and chloride-sulfate dominant. The dissolved solids concentration averaged $329 \mathrm{mg} / \mathrm{L}$ with a standard deviation of $197 \mathrm{mg} / \mathrm{L}$ and ranged from $57 \mathrm{mg} / \mathrm{L}$ (Quincy Creek) to $758 \mathrm{mg} / \mathrm{L}$ (Marco Island). The water hardness averaged $144 \mathrm{mg} / \mathrm{L}$ with a standard deviation of $68 \mathrm{mg} / \mathrm{L}$ and ranged from $33 \mathrm{mg} / \mathrm{L}$ (Quincy Creek) to $250 \mathrm{mg} / \mathrm{L}$ (St. Augustine).

The major inorganic chemical parameter concentrations between the raw and treated surface-water supplies were statistically tested for difference. Only the sulfate concentrations indicated a high level of significant difference. The sulfate concentrations indicated a significant difference at the 90 percent probability level with the treated water having the higher mean concentration. The explanation for the difference in sulfate concentration is not apparent, but it may simply be due to sampling chance. That is, the difference is significant only at the 90 percent probability level, thus there is a 10 percent probability that the concentrations are not significantly different, but the result of chance sampling or circumstance.

The Quality of Public Surface-Water Supplies with Reference to the National Interim Primary Drinking Water Regulations

In this report the quality of the treated water as it relates to the primary and secondary drinking water regulations is highlighted. It must be emphasized that while a single sample gives an approximation of parameter values, there does exist a certain probability of error or sample non-representativeness. Thus, single-sample interpretation does 
have obvious inherent limitations, and data are used mainly to identify situations where additional information is necessary.

of the trace elements covered by primary drinking water regulations only chromium and lead had concentrations near or exceeding the estab1ished MCL (maximum contaminant leve1) (table 3). The chromium concentration in the treated surface-water from the Manatee County Water System was $40 \mathrm{ug} / \mathrm{L}$ (micrograms per 1iter) or 80 percent of the $50 \mathrm{ug} / \mathrm{L}$ MCL. The treated supply for the city of Port St. Joe had a lead concentration of $61 \mathrm{ug} / \mathrm{L}$ which is 22 percent in excess of the established $50 \mathrm{ug} / \mathrm{L}$ MCL. The treated supplies for the city of Quincy and the Bay County Water System had lead concentrations of 49 and $44 \mathrm{ug} / \mathrm{L}$. The treated surface-water for Marco Island had a lead concentration of 30 ug/L with the remaining surface supplies having lead concentrations well below the established MCL.

Pesticide compounds for which drinking water regulations have been established were detected in two treated surface supplies, but the concentrations were well below the Federal MCL. A 2,4-D concentration of $0.09 \mathrm{ug} / \mathrm{L}$ was detected in the water supply for he city of Melbourne in Brevard County and $0.16 \mathrm{ug} / \mathrm{L}$ of silvex was detected in the North Port Charlotte water supply.

The concentrations of both fluoride and nitrate in the treated surface water supplies were well below their respective MCL. Turbidity levels averaged 1 NTU in the surface supplies with a maximum of 4 NTU for the city of Okeechobee in Okeecobee County. Of the 17 treated public surface-water supplies sampled only two had no detectable turbidity and 15 supplies had levels ranging between 1 and 4 NTU.

The results of the radioactivity analysis indicated that 16 of the 17 supplies sampled had gross alpha activity levels less than about 8 pCi/L (picocuries per liter) which is well below the $15 \mathrm{pCi} / \mathrm{L}$ Federal MCL. However, the water supply for Marco Island had a gross alpha activity of $19 \mathrm{pCi} / \mathrm{L}$.

Results of analyses for the primary drinking water regulation parameters in the untreated public surface-water supplies are summarized in table 4. The regulations do not directly pertain to the untreated water but the results are presented for comparison with the treated water (table 3 ). To evaluate in a very general way if water treatment has a significant effect on the concentrations a series of statistical tests were made between the mean concentrations for raw and treated water. Test results indicated that no significant difference in concentrations existed between the raw and treated water. Chromium, mercury, and gross radioactivity levels were not tested because many concentrations were less than the analytical detection limit. 
Table 3.--Summary of chemical and physical analyses of National Interim Primary Drinking Water Regulation parameters for selected treated public surface-water supplies.

[Total concentrations in micrograms per liter, except as indicated]

\begin{tabular}{|c|c|c|c|c|c|}
\hline Parameter & $\begin{array}{c}\text { Number of } \\
\text { samples }\end{array}$ & Mean & $\begin{array}{l}\text { Standard } \\
\text { deviation }\end{array}$ & Range & Median \\
\hline $\begin{array}{l}\text { Arsenic (As) } \\
\text { Barium (Ba) } \\
\text { Cadmium (Cd) } \\
\text { Chromium (Cr) } \\
\text { Lead (Pb) } \\
\text { Mercury (Hg) } \\
\text { Selenium (Se) } \\
\text { Silver (Ag) }\end{array}$ & $\begin{array}{r}16 \\
7 \\
17 \\
17 \\
17 \\
16 \\
16 \\
17\end{array}$ & $\begin{array}{l}1 \\
0 \\
0 \\
(1) \\
14 \\
(1) \\
0 \\
0\end{array}$ & $\begin{array}{r}1 \\
0 \\
1 \\
- \\
20 \\
0 \\
1\end{array}$ & $\begin{array}{c}0-3 \\
0 \\
0-2 \\
<10-40 \\
0-61 \\
<0.5 \\
0 \\
0-2\end{array}$ & $\begin{array}{c}0 \\
0 \\
0 \\
10 \\
3 \\
<.5 \\
0 \\
0\end{array}$ \\
\hline $\begin{array}{l}\text { Endrin } \\
\text { Lindane } \\
\text { Methoxychlor } \\
\text { Toxaphene } \\
2,4-D \\
\text { Silvex }\end{array}$ & $\begin{array}{l}17 \\
17 \\
17 \\
17 \\
16 \\
16\end{array}$ & $\begin{array}{l}.00 \\
.00 \\
.00 \\
0 \begin{array}{l}.01 \\
.01\end{array}\end{array}$ & $\begin{array}{l}.00 \\
.00 \\
.00 \\
0 \\
.02 \\
.04\end{array}$ & $\begin{array}{c}.00 \\
.00 \\
.00 \\
0 \\
.00-0.09 \\
.00-0.16\end{array}$ & $\begin{array}{r}.00 \\
.00 \\
.00 \\
0 \\
.00 \\
.00\end{array}$ \\
\hline $\begin{array}{l}\text { Fluoride (F) (mg/L) } \\
\left.\text { Nitrate ( } \mathrm{NO}_{3}-\mathrm{N}\right)(\mathrm{mg} / \mathrm{L}) \\
\text { Turbidity (NTU) }\end{array}$ & $\begin{array}{l}17 \\
17 \\
17\end{array}$ &.$_{1}^{.2}$ &.$_{1}^{.2}$ & $\begin{array}{l}.0-0.6 \\
.00-0.38 \\
0-4\end{array}$ &.$^{.13}$ \\
\hline Alpha, gross (pCi/L) & 17 & $\left({ }^{1}\right)$ & - & $<0.5-19$ & $<3.1$ \\
\hline
\end{tabular}

(3) Some concentrations were below the analytical detection limit. 
Table 4.--Summary of chemical and physical analyses of National Interim Primary Drinking Water Regulation parameters for selected untreated surface-water supplies.

[Total concentrations in micrograms per liter, except as indicated]

\begin{tabular}{|c|c|c|c|c|c|}
\hline Parameter & $\begin{array}{c}\text { Number of } \\
\text { samples }\end{array}$ & Mean & $\begin{array}{l}\text { Standard } \\
\text { deviation }\end{array}$ & Range & Median \\
\hline Arsenic (As) & 18 & 1 & 1 & $0-3$ & 1 \\
\hline Barium (Ba) & 8 & 0 & 100 & $0-100$ & 0 \\
\hline Cadmium (Cd) & 18 & 0 & 1 & $0-2$ & 0 \\
\hline Chromium (Cr) & 18 & $\left({ }^{1}\right)$ & - & $<10-20$ & 6 \\
\hline Lead $(\mathrm{Pb})$ & 18 & 14 & 16 & $0-62$ & 8 \\
\hline Mercury (Hg) & 18 & $\left({ }^{1}\right)$ & - & $<0.5$ & $<.5$ \\
\hline Selenium (Se) & 18 & 0 & 0 & 0 & 0 \\
\hline Silver (Ag) & 18 & 0 & 1 & $0-2$ & 0 \\
\hline Endrin & 17 & .00 & .00 & .00 & .00 \\
\hline Lindane & 17 & .00 & .00 & .00 & .00 \\
\hline Methoxychlor & 17 & .00 & .00 & .00 & .00 \\
\hline Toxaphene & 17 & 0 & 0 & 0 & 0 \\
\hline $2,4-D$ & 15 & .02 & .04 & $.00-0.16$ & .00 \\
\hline Silvex & 15 & .00 & .01 & $.00-0.05$ & .00 \\
\hline Fluoride $(\mathrm{F})(\mathrm{mg} / \mathrm{L})$ & 18 & .2 & .2 & $.0-0.6$ & .2 \\
\hline Nitrate $\left(\mathrm{NO}_{2}-\mathrm{N}\right)(\mathrm{mg} / \mathrm{L})$ & 16 & .13 & .11 & $.00-0.38$ & .13 \\
\hline Turbidity (सेT) & 16 & 2 & 1 & $1-5$ & 1 \\
\hline Alpha, gross (pCi/L) & 15 & $\left({ }^{1}\right)$ & -- & $<0.8-<8.2$ & $<3.2$ \\
\hline
\end{tabular}

( $\left.{ }^{1}\right)$ Some concentrations were below the analytical detection limit. 
The Quality of Public Surface-Water Supplies with Reference to the Proposed National Secondary Drinking Water Regulations

A summary of the secondary drinking water regulations parameters for the treated surface-water supplies is given in table 5 .

Several minor exceedences were found in the treated surface-water supplies with regard to the proposed secondary drinking water regulations. Some levels of chloride, color, dissolved solids, iron, and $\mathrm{pH}$ exceeded the recommended secondary regulations.

The maximum chloride concentration was $330 \mathrm{mg} / \mathrm{L}$ in the Marco Island water supply and exceeded the proposed $250 \mathrm{mg} / \mathrm{L}$ secondary 1 imit by 32 percent. The water supply for the city of Okeechobee had a color of 22 Pt-Co units; the recommended limit is 15 Pt-Co untts. Three public supplies exceeded the recommended $500 \mathrm{mg} / \mathrm{L}$ dissolved solids concentrations. The water supplies for West $\mathrm{Palm} B \mathrm{meach}$ and St. Augustine slightly exceeded the recommended limit having respective dissolved solid concentrations of $501 \mathrm{mg} / \mathrm{L}$ and $564 \mathrm{mg} / \mathrm{L}$, and the water supply for Marco Island had a dissolved solids concentration of $758 \mathrm{mg} / \mathrm{L}$. The Marco Island supply also had an iron concentration of $700 \mathrm{ug} / \mathrm{L}$ which is over twice the recommended limit of $300 \mathrm{ug} / \mathrm{L}$. The $\mathrm{pH}$ values of 8 of 17 treated public supplies were in excess of the 8.5 recommended limit with 6 ranging between 9.1 and 9.7 .

The analytical results for the secondary drinking water regulation parameter levels in the untreated surface-water supplies are summarized in table 6. The mean concentrations of the secondary drinking water parameters in raw and treated water were statistically tested to determine if significant concentration differences existed. Only color and iron were found to be significantly different at the 95 percent probability level. Both the level of color and iron were lower in the treated water than in the raw water. Most of the surface-water supply facilities use coagulation and filtration treatment which is the major cause of the significant reduction in color. Treatment plants also commonly use aeration and $\mathrm{pH}$ control along with coagulation, which undoubtedly are the controlling mechanisms reducing the concentration of iron in the treated water supplies.

\section{Public Ground-Water Supplies}

During the November 1977 to February 1978 reconnaissance, about 100 treated ground water public supplies were sampled throughout the State. Most of the supplies that were sampled were analyzed for the complete suite of primary and secondary regulation parameters are summarized in tables 7 and 8 . Some supplies were analyzed only for gross radioactivity. A list of the public supplies are given in table 2, and a complete data presentation for each public supply is given beginning on page 41 . 
Table 5.-- Summary of chemical and physical analyses of proposed National Secondary Drinking Water Regulation parameters for selected treated public surface-water supplies.

[Total concentrations in milligrams per liter, except as 1ndicated]

\begin{tabular}{|c|c|c|c|c|c|}
\hline Parameter & $\begin{array}{c}\text { Number of } \\
\text { samples }\end{array}$ & Mean & $\begin{array}{l}\text { St andard } \\
\text { deviation }\end{array}$ & Range & Median \\
\hline Chloride ( $\mathrm{Cl}$ ) & 17 & 88 & 85 & $\begin{array}{l}10-330 \\
0-2 ?\end{array}$ & 72 \\
\hline $\begin{array}{l}\text { Color (Pt-Co units) } \\
\text { Copper (Cu) } \\
\text { Dissolved solids }\end{array}$ & $\begin{array}{l}17 \\
17\end{array}$ & ${ }^{5} .012$ & ${ }^{6} .019$ & $\begin{array}{c}0-22 \\
0.001-0.069\end{array}$ & $9^{4} .004$ \\
\hline (residue at $180^{\circ} \mathrm{C}$ ) & 17 & 329 & 197 & $57-758$ & 354 \\
\hline Iron (Fe) & 17 & .100 & .160 & $.020-0.700$ & .050 \\
\hline Manganese (Mn) & 17 & .010 & .010 & $.00-0.020$ & .010 \\
\hline $\mathrm{pH}$ (units) & 17 & - & - & $7.1-9.7$ & 8.2 \\
\hline Sulfate $\left(\mathrm{SO}_{4}\right)$ & 17 & 73 & 42 & $6.4-160$ & 79 \\
\hline
\end{tabular}


Table 6.--Summary of chemical and physical analyses of proposed National Secondary Drinking Water Regulation parameters for selected untreated surface-water supplies.

[Total concentrations in milligrams per 11ter, except as indicated]

\begin{tabular}{|c|c|c|c|c|c|}
\hline Parameter & $\begin{array}{c}\text { Number of } \\
\text { samples }\end{array}$ & Mean & $\begin{array}{l}\text { Standard } \\
\text { deviation }\end{array}$ & Range & Median \\
\hline Chloride (C1) & 18 & 61 & 49 & $5.1-150$ & 50 \\
\hline Color (Pt-Co units) & 18 & 84 & 82 & $0-240$ & 50 \\
\hline Copper $(\mathrm{Cu})$ & 18 & .010 & .009 & $.002-0.026$ & .006 \\
\hline Dissolved solids & & & & & \\
\hline (residue at $180^{\circ} \mathrm{C}$ ) & 18 & 306 & 181 & $36-632$ & 310 \\
\hline Iron $(\mathrm{Fe})$ & 18 & .520 & .640 & $.020-2.7$ & .300 \\
\hline Manganese (Mn) & 18 & .010 & .010 & $.000-0.040$ & .010 \\
\hline $\mathrm{pH}$ (units) & 18 & -- & - & $6.0-9.5$ & 7.1 \\
\hline Sulfate $\left(\mathrm{SO}_{4}\right)$ & 18 & 48 & 29 & $.6-94$ & 45 \\
\hline Zinc $(2 n)$ & 18 & .030 & .040 & $.000-0.140$ & .010 \\
\hline
\end{tabular}


General Inorganic Chemical Composition

The average chemical composition of the treated ground water public supplies is calcium ( 54 percent) bicarbonate ( 54 percent) of the cations and anions, respectively. On the average, sodium and magneslum comprised 25 and 20 percent of the cations while chloride and sulfate comprised 28 and 18 percent of the anions. Calcium averaged $51 \mathrm{mg} / \mathrm{L}$ and ranged from $1.9 \mathrm{mg} / \mathrm{L}$ (South Walton County Utilities) to $120 \mathrm{mg} / \mathrm{L}$ (North Miam1, Dade County and Venice Gardens, Sarasota County). Bicarbonate averaged $151 \mathrm{mg} / \mathrm{L}$ ranging from $0.0 \mathrm{mg} / \mathrm{L}$ (South Walton County Ut1lities) to $350 \mathrm{mg} / \mathrm{L}$ (Apalachicola, Franklin County).

The average dissolved solids concentration of the treated ground water public supplies was $280 \mathrm{mg} / \mathrm{L}$. However, a large concentration variance did exlst as shown by the $180 \mathrm{mg} / \mathrm{L}$ standard deviation. The dissolved solids concentration ranged from $42 \mathrm{mg} / \mathrm{L}$ (Pace, Santa Rosa County) to $999 \mathrm{mg} / \mathrm{L}$ (Gifford, Indian River County). Hardness averaged $176 \mathrm{mg} / \mathrm{L}$ and ranged from $13 \mathrm{mg} / \mathrm{L}$ (South Walton County Ut1lities) to 480 $\mathrm{mg} / \mathrm{L}$ (Venice Gardens, Sarasota County).

The Quality of Public Ground-Water Supplies with Reference to the National Interim Primary Drinking Water Regulations

In a smal1 number of public ground water supplies lead and selentum were found in concentrations which exceeded the MCL's established in the primary drinking water regulations (table 7). Four supplies had lead concentrations exceeding the $50 \mathrm{ug} / \mathrm{L}$ MCL: $52 \mathrm{ug} / \mathrm{L}$ Navarre Beach, Santa Rosa County; $68 \mathrm{ug} / \mathrm{L}$ South Miami Helghts, Dade County; 77 ug/L Woodville, Leon County; $80 \mathrm{ug} / \mathrm{L}$ Pace, Santa Rosa County. Selenfum was detected in only 8 of the 92 ground water supplies with 1 Federal MCL exceedence. The treated supply for the city of Wildwood, Sumter County, had a selenfum concentration of $15 \mathrm{ug} / \mathrm{L}$ exceeding the established selenium MCL of $10 \mathrm{ug} / \mathrm{L}$. In addition, while the water supplies for Colonial Hills and Forest Hills in Pasco County did not exceed the selenium MCL, they did contain concentrations of 6 and $4 \mathrm{ug} / \mathrm{L}$ respectively.

Cadmium, chromium, and mercury were not found in concentrations exceeding the primary drinking water MCL's, but several supplies had concentrations which approached the allowable levels. The water supplies for South Miamt Heights in Dade County and Woodville in Leon County had respective cadmium concentrations of 8 and $5 \mathrm{ug} / \mathrm{L}$. The established cadmium MCL is $10 \mathrm{ug} / \mathrm{L}$. A chromium concentration of $40 \mathrm{ug} / \mathrm{L}$ which is 80 percent of the $50 \mathrm{ug} / \mathrm{L}$ MCL was detected in the water supply for the city of Panacea, Wakulla County. The water supply samples collected for Eustis, Lake County, and Belleview in Marion County had mercury concentrations of 1.7 and $1.8 \mathrm{ug} / \mathrm{L}$ respectively. The MCL for mercury is $2.0 \mathrm{ug} / \mathrm{L}$. 
Table 7.--Summary of chemical and physical analyses of National Interim Primary Drinking Water Regulation parameters for selected treated public ground-water supplies.

[Total concentrations in micrograms per 1iter, except as indicated]

\begin{tabular}{|c|c|c|c|c|c|}
\hline Parameter & $\begin{array}{c}\text { Number of } \\
\text { samples }\end{array}$ & Mean & $\begin{array}{l}\text { Standard } \\
\text { deviation }\end{array}$ & Range & Median \\
\hline $\begin{array}{l}\text { Arsenfic (As) } \\
\text { Barlum (Ba) } \\
\text { Cadmium (Cd) } \\
\text { Chromfum (Cr) } \\
\text { Lead (Pb) } \\
\text { Mercury ( } \mathrm{Hg}) \\
\text { Selenium ( } \mathrm{Se}) \\
\text { Silver (Ag) }\end{array}$ & $\begin{array}{l}97 \\
43 \\
99 \\
99 \\
99 \\
98 \\
96 \\
98\end{array}$ & $\begin{array}{l}1 \\
0 \\
1 \\
(1) \\
14 \\
(1) \\
0 \\
0\end{array}$ & $\begin{array}{r}1 \\
0 \\
1 \\
-- \\
15 \\
-- \\
2 \\
1\end{array}$ & $\begin{array}{c}0-10 \\
0-100 \\
0-8 \\
<10-40 \\
0-80 \\
<0.5-1.8 \\
0-1.8 \\
0-2\end{array}$ & $\begin{array}{c}1 \\
0 \\
0 \\
10 \\
8 \\
<.5 \\
0 \\
0\end{array}$ \\
\hline $\begin{array}{l}\text { Endrin } \\
\text { Lindane } \\
\text { Methoxychlor } \\
\text { Toxaphene } \\
2,4-D \\
\text { Silvex }\end{array}$ & $\begin{array}{l}97 \\
96 \\
96 \\
96 \\
97 \\
97\end{array}$ & $\begin{array}{l}.00 \\
.00 \\
.00 \\
0 \\
.00 \\
.02\end{array}$ & $\begin{array}{l}.00 \\
.00 \\
.00 \\
0 \\
.01 \\
.1 .5\end{array}$ & $\begin{array}{l}.00 \\
.00 \\
.00 \\
0 \\
.00-0.04 \\
.00-1.4\end{array}$ & $\begin{array}{l}.00 \\
.00 \\
.00 \\
0 \\
.00 \\
.00\end{array}$ \\
\hline $\begin{array}{l}\text { Fluoride }(\mathrm{F})(\mathrm{mg} / \mathrm{L}) \\
\text { Nitrate }\left(\mathrm{NO}_{3}-\mathrm{N}\right)(\mathrm{mg} / \mathrm{L}) \\
\text { Turbidity (NTU) }\end{array}$ & $\begin{array}{l}97 \\
97 \\
95\end{array}$ &.$_{1}^{.38}$ &.$_{1}^{.4}$ & $\begin{array}{l}.0-3.3 \\
.00-5.8 \\
0-6\end{array}$ & $1^{.04}$ \\
\hline Alpha, gross (pCi/L) & 74 & $\left({ }^{1}\right)$ & - & $<0.9-41$ & 3.6 \\
\hline
\end{tabular}

( ${ }^{1}$ Some concentrations were below the analytical detection limit. 
Pesticide compounds, 2,4-D and silvex, were detected in trace quantities in a few public supplies, but none approximated the National Interim Primary Drinking Water Regulation MCL's. The maximum concentration of 2,4-D was $0.04 \mathrm{ug} / \mathrm{L}$ detected in the treated water supply for Indiantown, Martin County. Four additional supply waters had a detectable 2,4-D concentration of $0.02 \mathrm{ug} / \mathrm{L}$ : Villa Del Ray, Palm Beach County; First Florida Utilities, Palm Beach County; Venice Gardens, Sarasota County; and Port St. Lucie, St. Lucie County. The maximum silvex concentration was $1.4 \mathrm{ug} / \mathrm{L}$ in the treated water for Sunrise, Broward County. Other public supplies which had detectable levels of silvex were Collier Estates, Broward County, 0.14 ug/L; Tamarac, Broward County, $0.06 \mathrm{ug} / \mathrm{L}$; and Port St. Lucle, St. Lucle County, $0.02 \mathrm{ug} / \mathrm{L}$.

A concentration of 1.4 to $1.6 \mathrm{mg} / \mathrm{L}$ is the MCL for fluoride in areas having an annual average maximum daily alr temperature ranging from $70.7 \mathrm{~F}$ to $90.5 \mathrm{~F}$. Of the 97 public supplies analyzed during the reconnaissance only one exceeded the recommended limit. The treated supply for the city of Mexico Beach, Bay County, had a concentration of 3.3 $\mathrm{mg} / \mathrm{L}$. Two supplies, Navarre Beach (Santa Rosa County) and Zolfo Springs (Hardee County) contained fluoride concentration of $1.0 \mathrm{mg} / \mathrm{L}$, and the remalning supplies had concentrations of less than $1.0 \mathrm{mg} / \mathrm{L}$.

None of the treated public ground water supplies had nitrate nitrogen concentrations exceeding about 60 percent of the established $10 \mathrm{mg} / \mathrm{L}$ MCL. The water supplies for Forest Hills and Colonial Hills in Pasco County contained respective nitrate nitrogen concentrations of 5.8 and $5.7 \mathrm{mg} / \mathrm{L}$. Ninety-two of the 97 supplies had nitrate nitrogen concentrations ranging from 0.0 to $1.7 \mathrm{mg} / \mathrm{L}$ with 3 ranging from 2.3 to 2.9 $\mathrm{mg} / \mathrm{L}$.

The turbidity level in all treated waters was 3 NTU or less except for South Walton County Utilities with 6 NTU and Navarre Beach in Santa Rosa County with 4 NTU. Seventy-six supplies had turbidities between 1 and 3 with 17 having no detectable turbidity.

Five of the 74 treated water supplies analyzed for gross alpha radioactivity had levels greater than the Federally established $15 \mathrm{pCi} / \mathrm{L}$ MCL. The supplies were: $41 \mathrm{pCi} / \mathrm{L}$ Colonial Hills (Pasco County); 33 $\mathrm{pCl} / \mathrm{L}$ Venice Gardens (Sarasota County); $20 \mathrm{pCi} / \mathrm{L}$ Zolfo Springs (Hardee County); $19 \mathrm{pCi} / \mathrm{L}$ Apalachicola (Franklin County); and $17 \mathrm{pC1} / \mathrm{L}$ Gifford (Indian River County). Additionally, 4 supplies had gross alpha activities ranging from 10 to $14 \mathrm{pCi} / \mathrm{L}$.

The Quality of Public Ground-Water Supplies with Reference to the Proposed National Secondary Drinking Water Regulations

The analytical results of selected treated ground-water supplies for the secondary regulation parameters are summarized in table 8. Exceedences occurred occasionally for all secondary parameters except 
Table 8.--Summary of chemical and physical analyses of proposed National Secondary Drinking Water Regulation parameters for selected treated public ground-water supplies.

[Total concentrations in milligrams per liter, except as indicated]

\begin{tabular}{|c|c|c|c|c|c|}
\hline Parameter & $\begin{array}{c}\text { Number of } \\
\text { samples }\end{array}$ & Mean & $\begin{array}{l}\text { Standard } \\
\text { deviation }\end{array}$ & Range & Median \\
\hline Chloride (C1) & 98 & 46 & 57 & $2.5-310$ & 21 \\
\hline Color (Pt-Co units) & 98 & 4 & 6 & $0-42$ & 2 \\
\hline Copper (Cu) & 99 & .019 & .032 & $0.001-0.160$ & .007 \\
\hline Dissolved solids & & & & & \\
\hline (residue at $180^{\circ} \mathrm{C}$ ) & 98 & 280 & 180 & 42-999 & 225 \\
\hline Iron $(\mathrm{Fe})$ & 99 & .115 & .257 & $.000-2.40$ & .050 \\
\hline Manganese (Mn) & 99 & .010 & .010 & $.000-0.050$ & .000 \\
\hline $\mathrm{pH}$ (units) & 104 & -- & - & $5.5-9.3$ & 7.5 \\
\hline Sulfate $\left(\mathrm{SO}_{4}\right)$ & 98 & 39 & 65 & $.1-300$ & 11 \\
\hline Zinc $(Z n)$ & 98 & .070 & .140 & $.000-0.830$ & .020 \\
\hline
\end{tabular}


copper, manganese, and zinc. The maximum copper concentration was 0.16 $\mathrm{mg} / \mathrm{L}$ in the water supply for the city of Weeki Wachee in Hernando County and the maximum zinc concentration was $0.83 \mathrm{mg} / \mathrm{L}$ in the supply for Holly Hill in Volusia County. The proposed MCL for copper and zinc are 1.0 and $5.0 \mathrm{mg} / \mathrm{L}$ respectively.

Only one chloride concentration exceeded the proposed Federal limit. The chloride concentration in the supply for North Miami, Dade County, was $310 \mathrm{mg} / \mathrm{L}$. which is 24 percent greater than the $250 \mathrm{mg} / \mathrm{L}$ 1imit. Three additional supplies, Rotonda West (Charlotte County), Anastasia (St. Johns County), and Gifford (Indian River County) had concentrations exceeding $200 \mathrm{mg} / \mathrm{L}$. The sulfate concentrations in two public supplies were above the proposed $250 \mathrm{mg} / \mathrm{L}$ level. The supply for the G. Pierce Memorial Hospital (De Soto County) had a sulfate concentration of $260 \mathrm{mg} / \mathrm{L}$ and the city of Ruskin (Hillsborough County) had a concentration of $300 \mathrm{mg} / \mathrm{L}$. Ten public water supplies had dissolved solid concentrations exceeding the proposed $500 \mathrm{mg} / \mathrm{L}$ Federal limit. The maximum dissolved solids concentration in the treated ground water was $999 \mathrm{mg} / \mathrm{L}$ and was detected in the supply for the city of Gifford (Indian River County). Other public supplies exceeding the $500 \mathrm{mg} / \mathrm{L}$ dissolved solids MCL were: North Miami (Dade County), $924 \mathrm{mg} / \mathrm{L}$; Colonial Hills (Pasco County); $524 \mathrm{mg} / \mathrm{L} ;$ G. Pierce Memorial Hospital (De Soto County), $544 \mathrm{mg} / \mathrm{L}$; Mexico Beach (Bay County), $610 \mathrm{mg} / \mathrm{L}$; Ruskin (Hillsborough County), $629 \mathrm{mg} / \mathrm{L}$; Apalachicola (Frank1in County), $693 \mathrm{mg} / \mathrm{L}$; Anastasia (St. Johns County), $750 \mathrm{mg} / \mathrm{L}$; Venice Gardens (Sarasota County), 800 $\mathrm{mg} / \mathrm{L}$; and Branford (Suwannee County), $545 \mathrm{mg} / \mathrm{L}$.

Color exceeded the proposed secondary drinking water 11mit of 15 Pt-Co units in 6 treated public ground-water supplies. The maximum exceedence was $42 \mathrm{Pt}-\mathrm{Co}$ units in water from the First Florida Utilities system near $\mathrm{Palm}$ Beach ( $\mathrm{Palm}$ Beach County). Other exceedences were: 22 Pt-Co units, Davie (Broward County); 20 Pt-Co units, Fort Myers (Lee County); 27 Pt-Co units, North Miami (Dade County); 18 Pt-Co units, Port St. Lucie (St. Lucie County); 15 Pt-Co units, Tamarac (Broward County).

Five water supplies had iron concentrations which exceeded the 0.3 $\mathrm{mg} / \mathrm{L}$ limit. South Walton County Utilities supply water contained 2.4 $\mathrm{mg} / \mathrm{L}$ of iron, Tamarac in Broward County had $0.64 \mathrm{mg} / \mathrm{L}$, San Antonio in Pasco County had $0.55 \mathrm{mg} / \mathrm{L}$, Apalachicola in Franklin County had 0.33 $\mathrm{mg} / \mathrm{L}$, and Manalapan in Palm Beach County had $0.47 \mathrm{mg} / \mathrm{L}$.

The $\mathrm{pH}$ in 8 treated public ground-water supplies was outside the proposed range of 6.5 to 8.5 . Seven supplies had values above 8.5 with 1 below 6.5. The water supplies ranging between 8.6 and 9.3 were North Miami, Fort Myers, Englewood, G. Pierce Wood Memorlal Hospital, Holly H111, Flagler Beach, and Anastasia. The water from the South Walton County Utilities had a $\mathrm{pH}$ of 5.5 . 


\section{Frequency Distribution of the National Interim Primary Drinking Water Regulation Parameters}

A serles of histograms giving concentration ranges for selected parameters as a function of the number of treated public water supplies are given in figure 5. Both the treated surface and ground-water supplies are included in this summary to depict general conditions statewide.

Statewide, the concentrations of arsenic, barium, cadmium, mercury, and silver in Florida's treated drinking water meet the Federal regulations with a large percentage of the supplies having no detectable levels of these parameters. For example, no arsenic was detected in 46 of the 113 supplies analyzed, no barium was detected in 45 of the 50 supplies analyzed, no cadmium was detected in 79 of the 116 supplies analyzed, and no silver was detected in 88 of the 115 supplies analyzed. The concentrations of chromium ranged from $<10$ to $10 \mathrm{ug} / \mathrm{L}$ in 81 percent (94) of the supply waters. Eighty-nine percent (102) of the supplies had a mercury concentration of $<0.5 \mathrm{ug} / \mathrm{L}$.

Lead and selenium levels in some supplies were above Federal MCL's; although, this was not a frequent occurrence. Of the selenium analyses, 93 percent (104) of the supplies were $0 \mathrm{ug} / \mathrm{L}$; however, one public supply had a MCL exceedence of $15 \mathrm{ug} / \mathrm{L}$. Sixty-three percent (73) of the supplies had lead concentrations ranging from 6 to $49 \mathrm{ug} / \mathrm{L}$ with 14 percent (16) of the supplies having a concentration of $0 \mathrm{ug} / \mathrm{L}$, and 4 percent (5) of the supplies having a concentration exceeding the $50 \mathrm{ug} / \mathrm{L}$ MCL.

Traces of pesticlde compounds were detected in 11 percent (12) of the public supplies, but the distribution was highly skewed with 89 percent (102) of the water supplies having no detectable pesticides. The herbiclde compounds, 2,4-D or silvex, were found in 10 of the supplies. The insecticide compounds, dieldrin and DDT, each were detected in one public supply.

Virtually all the nitrate and fluoride concentrations in the treated water supplies were below the Federal MCL's. Seventy-one percent (81) of the water supplies had fluoride concentrations ranging from 0.1 to $0.4 \mathrm{mg} / \mathrm{L}$. The distribution of nitrate was somewhat more unimodal than fluoride with about 52 percent (59) of the supplies having a concentration range between 0.01 and $0.1 \mathrm{mg} / \mathrm{L}$.

Turbidity levels in 111 of the 112 treated supply waters were below the 5 NTU Federal MCL. Seventy percent (78) of the supplies had a turbidity of 1 NTU, 17 percent (19) of the supplies had 0 NTU, and 4 percent (5) of the supplies had turbidities ranging between 3 and 6 NTU. 


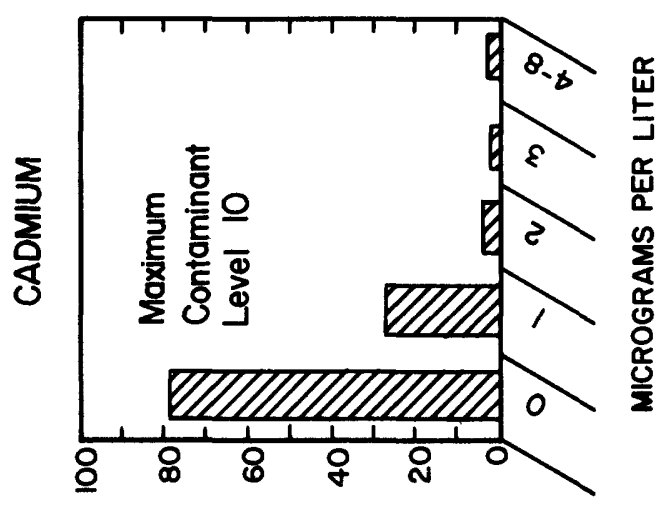

S $317 d d$ ก गา

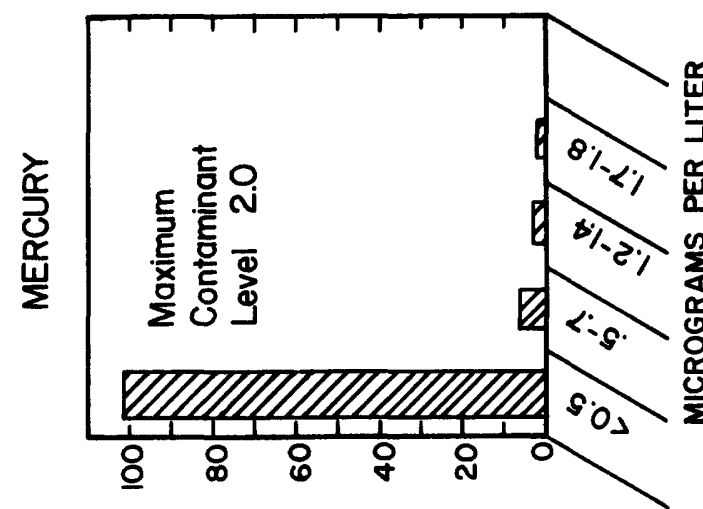

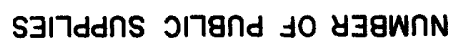
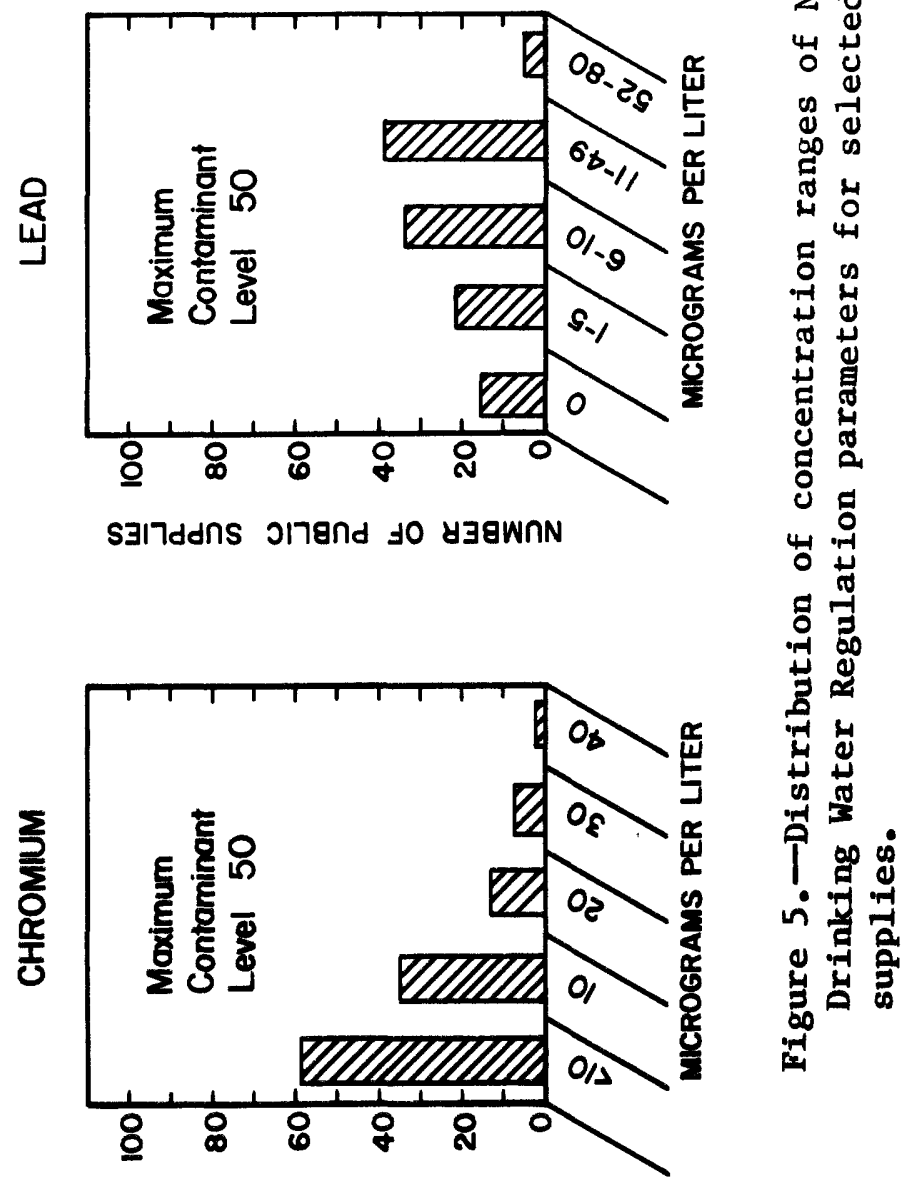

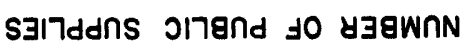

S3I7ddns गารกd to y3EWnN

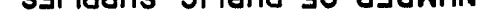

s3iาddกs गาgกd so y3ewnN

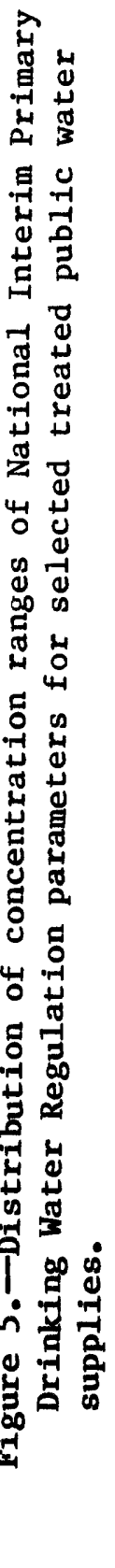

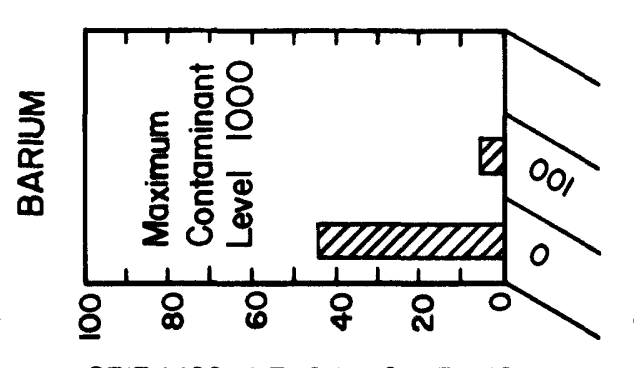

s3iาddns गาend to y3ewnN

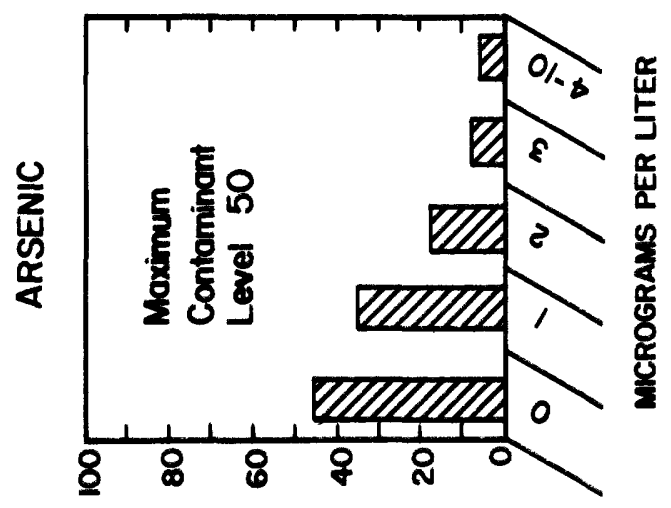

salnddns गาend to y3gwnN

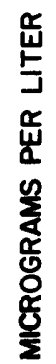



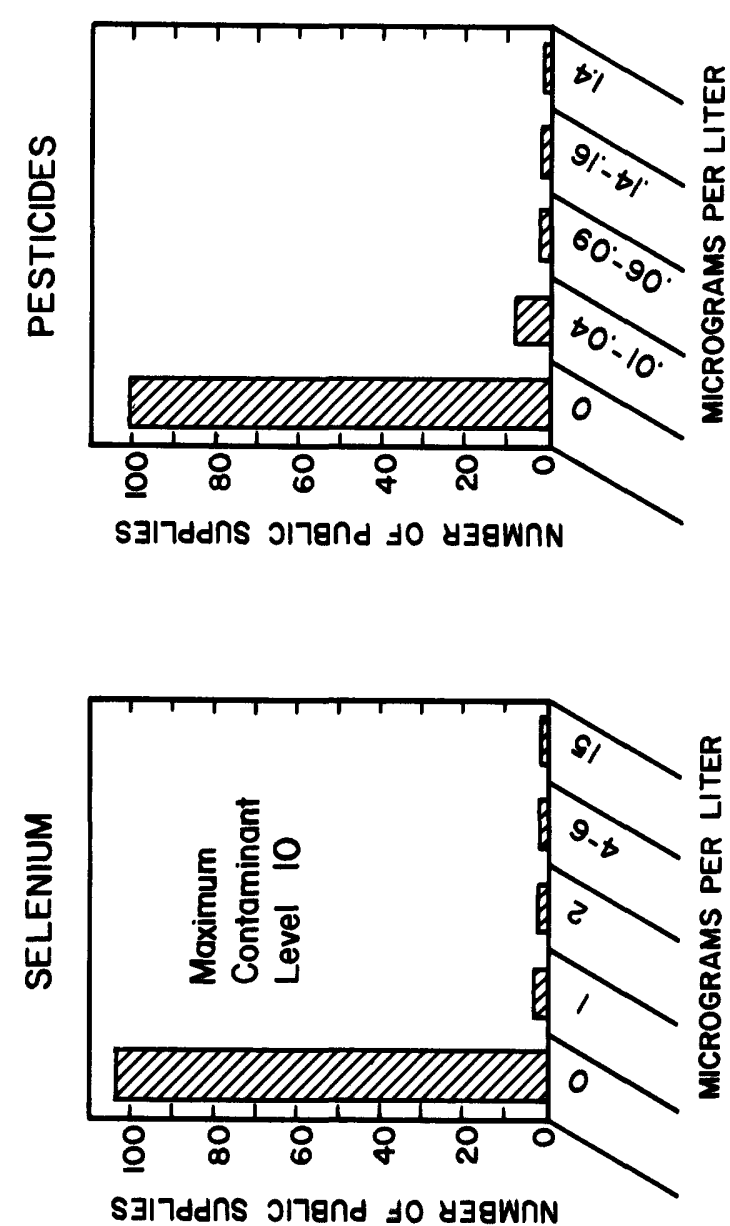

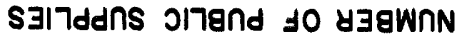

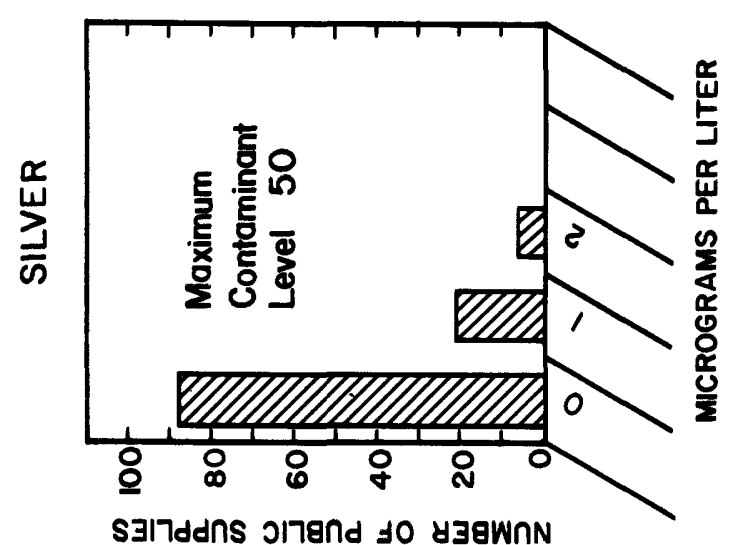

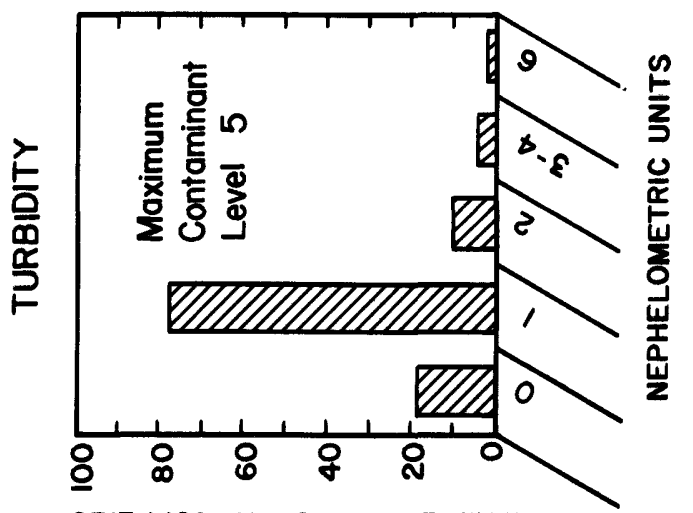

杯

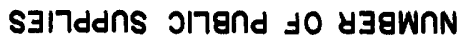

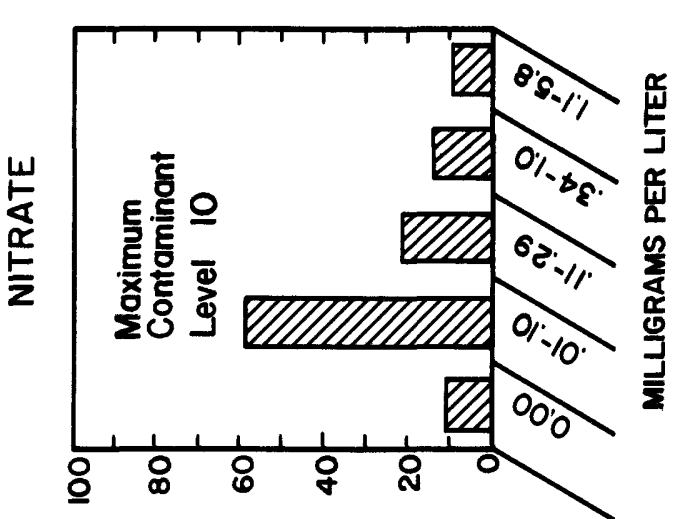

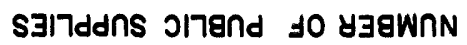

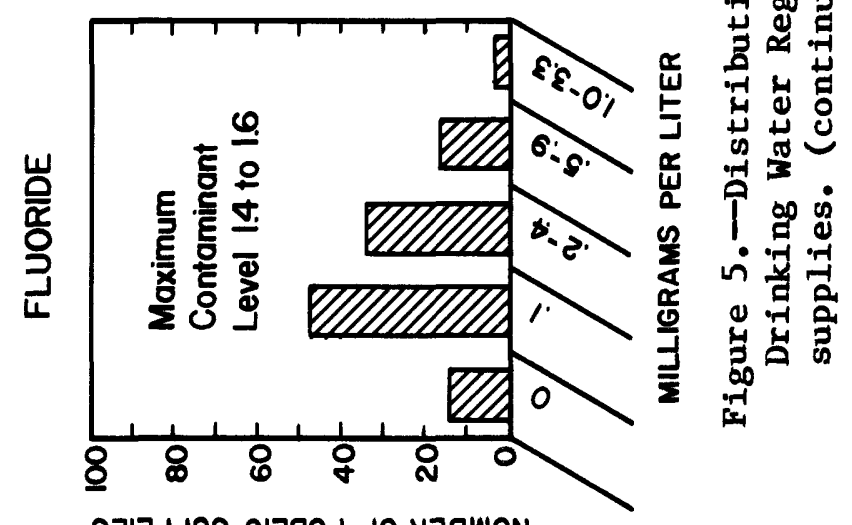

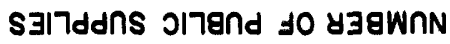




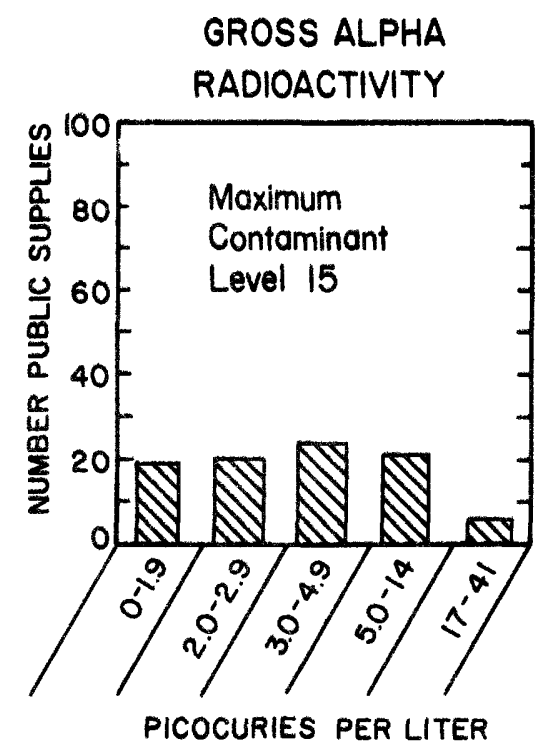

Figure 5.--Distribution of concentration ranges of National Interim Primary Drinking Water Regulation parameters for selected treated public water supplies. (continued) 
Seven percent $(6)$ of the treated water supplies sampled had gross alpha activities in excess of the $15 \mathrm{pC1} / \mathrm{L}$ Federal MCL and approximately 23 percent (21) of the supplies had activities ranging from 5 to 14 $\mathrm{pC}$ //L. It should be noted that 5 of the 21 supplies in the 5 to 14 $\mathrm{pCi} / \mathrm{L}$ range had alpha activities reported as "less than" because of analytical constraints and thus these 5 supplies may actually have activities below $5 \mathrm{pCl} / \mathrm{L}$. Seventy percent (63) of the water supplies were well within the gross alpha MCL.

\section{Frequency Distribution of the Proposed National Secondary Drinking Water Regulation Parameters}

The frequency distributions for the secondary drinking water regulation parameters are given in figure 6. As with the previous section, both the treated surface and ground-water supplies are included in this summary.

The chloride concentration in 96 (83 percent) of the 115 water supplies was less than $100 \mathrm{mg} / \mathrm{L}$. In only two water supplies was the chloride concentration in excess of the proposed $11 \mathrm{mit}$ of $250 \mathrm{mg} / \mathrm{L}$. Sulfate in the water supplies had a wide range in concentration, but only two exceeded the $250 \mathrm{mg} / \mathrm{L}$ proposed 1 imit.

Fifty-seven percent (65) of the 114 supplies analyzed had dissolved solid concentrations ranging from 42 to $251 \mathrm{mg} / \mathrm{L}$. However, the $500 \mathrm{mg} / \mathrm{L}$ proposed limit was exceeded in 12 of the supplies with concentrations ranging from 501 to $924 \mathrm{mg} / \mathrm{L}$.

The concentrations of copper, manganese, and zinc in the treated public water supplies did not exceed their recommended limits. Iron, however, exceeded the proposed limit of $300 \mathrm{ug} / \mathrm{L}$ in 6 of the supplies sampled.

Ninety-six percent (111) of the copper concentrations were below 84 $\mathrm{ug} / \mathrm{L}$ and 88 percent (101) of the zinc concentrations were $100 \mathrm{ug} / \mathrm{L}$ or less. The concentration of manganese in one water supply was $50 \mathrm{ug} / \mathrm{L}$, which is the proposed limit, but the concentrations in 97 percent (112) of the water supplies ranged from 0 to $20 \mathrm{ug} / \mathrm{L}$.

Color equaled or exceeded the $15 \mathrm{Pt}-\mathrm{Co}$ unit proposed 1 imit in 7 of the 115 public supplies. However, 77 percent (88) of the supplies had color levels between 0 and 5 Pt-Co units.

The values of $\mathrm{pH}$ malnly fell between 7.0 and 7.5 with 77 percent (93) of the supplies having $a \mathrm{pH}$ between 7.0 and 8.0 . But 15 supplies had $\mathrm{pH}$ values between 8.6 and $9.7 ; 8.6$ is the proposed upper 1 imit for drinking water. One supply had a pH value of 5.5 with 6.5 being the proposed lower limit. 

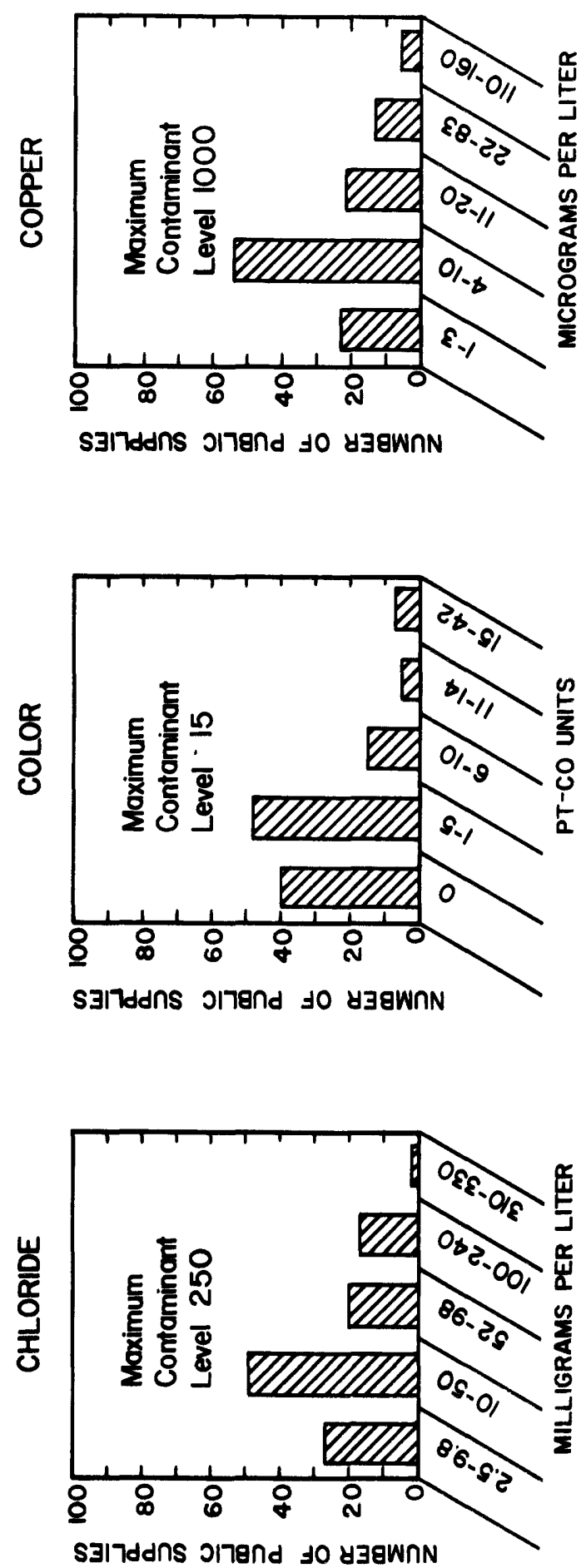
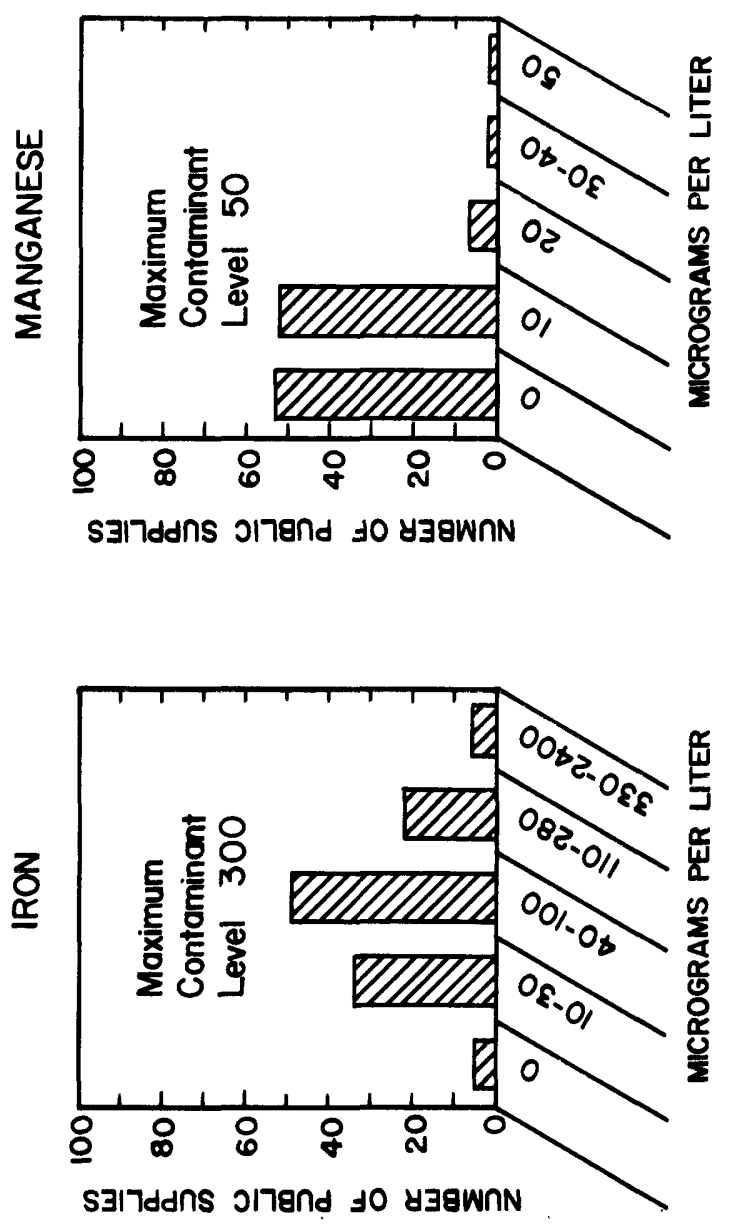

मु

ర్d

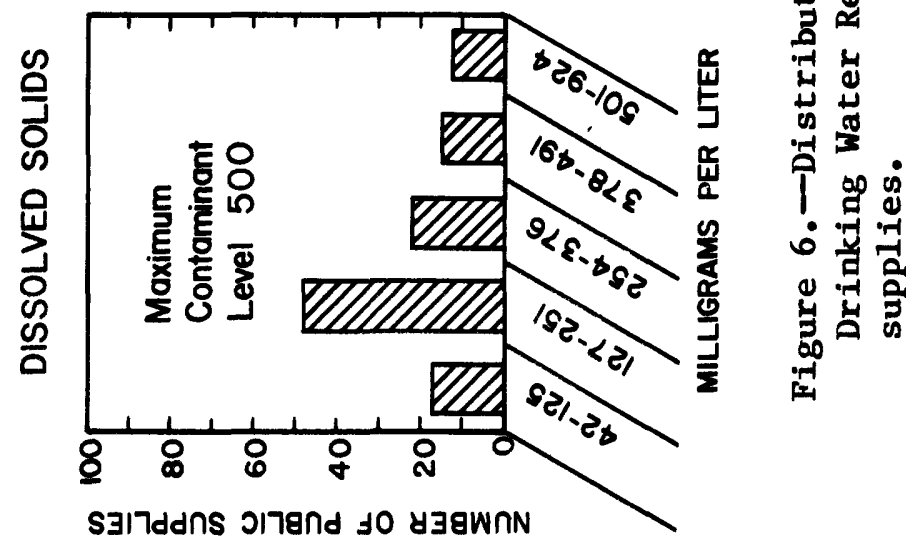



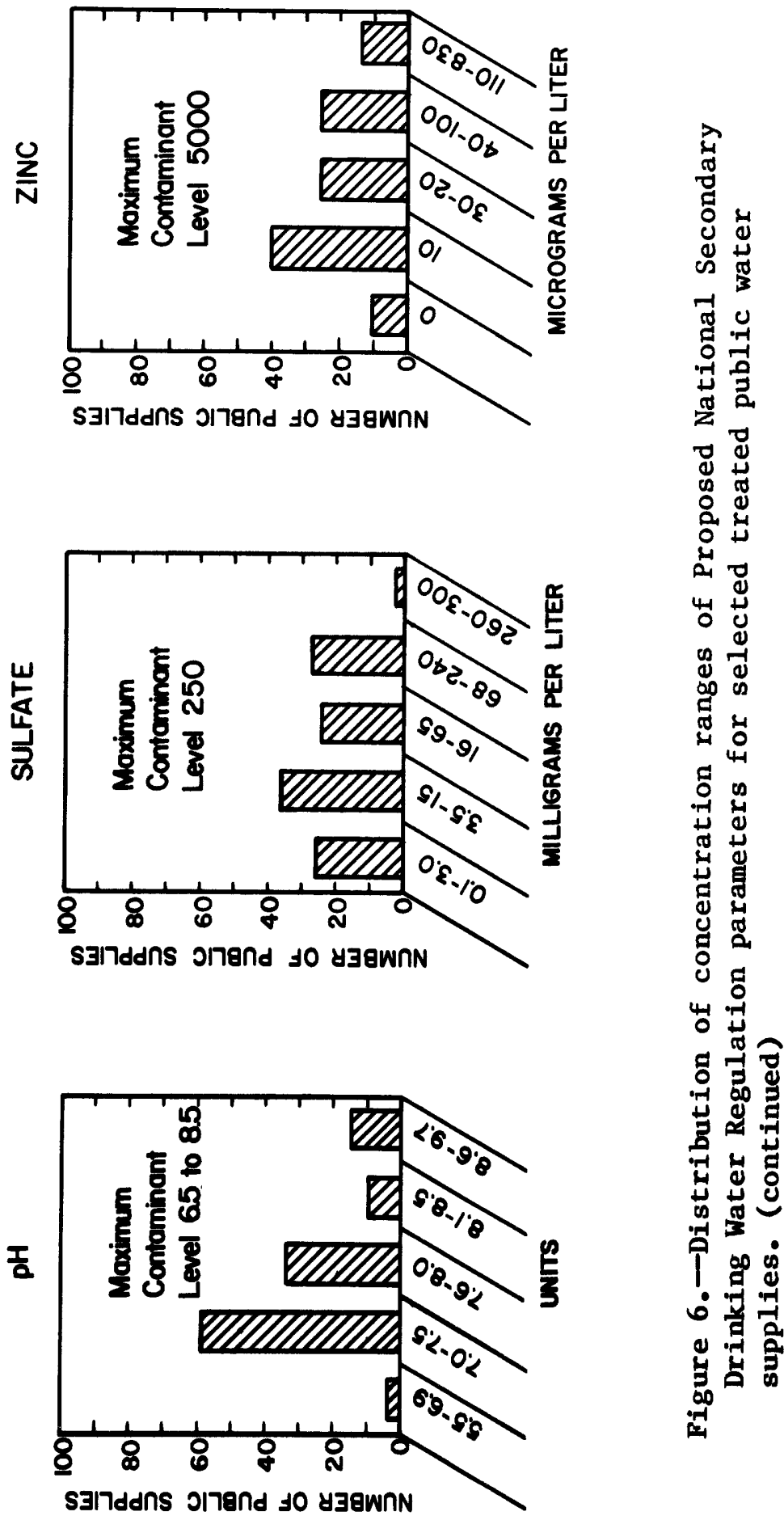


\section{SUMMARY}

The data included in this report, along with those reported by Irwin and Healy (1978), document the quality of drinking water in over 260 public supplies throughout Florida. These data reveal that virtually all the State's drinking water meets Federal requirements.

Statewide, the chemical composition of the treated public supply water is predominantly calcium bicarbonate. The average dissolved solids concentration is about $300 \mathrm{mg} / \mathrm{L}$ with an average water hardness of $160 \mathrm{mg} / \mathrm{L}$.

Specifically addressing the $1977-78$ water-quality sampling reconnaissance, a small number of primary drinking water regulation exceedences did occur at a few locations. For example, five public supplies had lead concentrations exceeding the MCL of $50 \mathrm{ug} / \mathrm{L}$, and 1 supply had a selenium concentration of $15 \mathrm{ug} / \mathrm{L}$ which is $5 \mathrm{ug} / \mathrm{L}$ over the MCL.

Trace concentrations of certain pesticide compounds were detected in 11 percent of the water supplies sampled. However, none of the 12 supplies had concentrations even near the Federal pesticide MCL. The herbicides, 2,4-D, and silvex, were the most frequently detected compounds.

The nitrate, fluoride, and turbidity levels in virtually all the public-supply waters were below the Federal MCL's. However, the water from 1 supply had a fluoride concentration of $3.3 \mathrm{mg} / \mathrm{L}$ and 1 supply had a turbidity of 6 NTU both of which are slightly above their respective Federal MCL.

Six treated public supplies had gross alpha radioactivity above the Federal MCL of $15 \mathrm{pCl} / \mathrm{L}$. Also between 16 and 21 supplies had alpha activity levels ranging between 5 and $14 \mathrm{pCi} / \mathrm{L}$.

In addition to the primary drinking water regulation parameters, some of the water supplies had excessive levels for some parameters identified in the Proposed National Secondary Drinking Water Regulations. For example, 12 supplies had dissolved solid concentrations exceeding the proposed limit of $500 \mathrm{mg} / \mathrm{L}$. Two supplies had chloride concentrations in excess of the recommended $250 \mathrm{mg} / \mathrm{L}$, and two supplies had sulfate levels above the recommended $250 \mathrm{mg} / \mathrm{L}$ 1imit. Iron concentrations exceeded the $300 \mathrm{ug} / \mathrm{L}$ proposed limit in 6 supplies with a maximum concentration of $2,400 \mathrm{ug} / \mathrm{L}$ occurring in an acidic water supply in northwestern Florida.

Color levels in 7 of the 115 public supplies equaled or exceeded the 15 Pt-Co unit proposed limit. Seventeen public supplies had pH levels outside the recommended limit of 6.5 to 8.5 . One supply had an acidic $\mathrm{pH}$ of 5.5 with $15^{\circ}$ supplies having $\mathrm{pH}$ values ranging from 8.6 to 9.7. 


\section{REFERENCES CITED}

Brown, Eugene, Skougstad, M. W., and Fishman, M. J., 1970, Methods for collection and analysis of water samples for dissolved minerals and gases: U.S. Geological Survey Techniques of Water-Resources Investigations, book 3, chapter Al, $160 \mathrm{p}$.

Fishman, M. J., and Brown, Eugene, 1976, Selected methods of the U.S. Geological Survey for the analysis of waste water: U.S. Geological Survey Open-file Report 76-177, 87 p.

Goerlitz, D. F., and Brown, Eugene, 1972, Methods for analysis of organic substances in water: U.S. Geological Survey Techniques of Water-Resources Investigations, book 5, chapter A3, $40 \mathrm{p}$.

Irwin, G. A., and Healy, H. G., 1978, Chemical and physical quality of selected public water supplies in Florida, August-September 1976: U.S. Geological Survey Water-Resources Investigations 78-21, $200 \mathrm{p}$.

U.S. Congress, 1974, Public Law 93-523, Safe Drinking Water Act: Washington D. C.

U.S. Environmental Protection Agency, 1974, Methods for chemical analysis of water and waste: National Environmental Research Center, Cincinnati, Ohio, 298 p. 1975, National interim primary drinking water regulations:

Federal Register, v. 40, no. 51, March 14, p. 11990-11998. 1975, National interim primary drinking water regulations:

Federal Register, v. 40, no. 248, Wednesday, December 24, 1975, Part IV, p. 59566-59587.

1976, Interim primary drinking water regulations - promulgation of regulations on radionuclides: Federal Register, v. 41, no. 133, Friday, July 9, 1976, Part II, p. 28402-29409.

1976, Guidelines establishing test procedures for the analysis of pollutants: Federal Register, v. 41, no. 232, December 1, p. 52780-52786.

1977, National secondary drinking water regulations: Federal Register, v. 42, no. 62, Thursday, March 31, 1977, Part I, p. 17143-17147.

U.S. Public Health Service, 1962, Drinking water regulations, 1962: Publication 956, 61 p. 
The following data compilation is presented as a user-reference document for specific sampling sites. A brief statement explaining specific item headings is given below:

Public Water Supply.--Identifies the public water supply and community served.

County.--The county in which the public water supply is located.

Collection Date. - The date the water sample was collected.

Sampling Point.--Identifies the location of sample collection. The 15 digit number is the latitude and longitude. Al 1 samples are identified in the U.S. Geological Survey Water Resources Division (WRD) data file using the specific source parameter code, 72005: The raw water-public water supply (untreated) samples are identified by a value of 46; the treated water--public water supply (treated) samples are identified by a value of 44 . 
Anastasia Sanitary District-Apalachicola---_-----_-_---Arcadia-_-_-_Atlantic Beach------_------

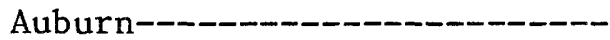
Avon Park-_-_-_-_-_-_-Baker---_Bay County Water System-----Bellair--

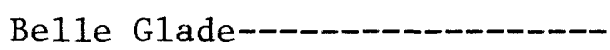

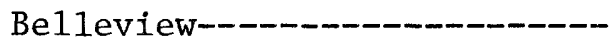
Bradenton--_-_-_-_-_-_-_-Branford-_Bunne11 Ca1lahan---_Carrolwood--_-_-_-_-_-Century Village Utilities---Chat tahoochee (Florida State Hospita1) -

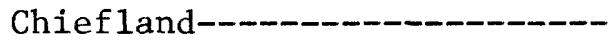

Cle rmont-_-_-_-_-_-_-_-_

Clewiston----_--_---_------

Collier Estates----

Colonial Hills------------

Conway (First Florida Utilities)-_-

Crescent City--_---_---

Crystal River---_---_------

Davie-----------------------

Delmar Corporation-----------

Deltona---_-

Eaton Park-_-

Englewood-----_----------

Eustis--_-_-

Fernandina Beach-_-_-_-_-_-

First Florida Utilities-----

Flagler Beach---_--_-------

F1orida City-_-_-_-_-_--_---

Forest Hills-------------

Fort Meade-_-_-_-_-_-_-_-_

Frostproof-- -

G. Pierce Wood Memorial

Hospita1-_-

Garden Grove---_--_-----

Gifford-- - -

Graceville---_-_-_-_----

Greenville--_-_-_-_-_-_-_-_

Groveland-
41
Havana----_-_-_-_-

Hilliard-_-_-_-_-_-_-_-

Hobe Sound--_-_-_-_-_---

Holiday Lakes Estates--_-----

Holly Hi11-_-

Hudson Community Water Works-

Immokalee-_-

Imperial Lakes-------_-------

Indian Hills-_-_-_-_-_-_-

Indiantown--_-

Interlachen-_-_-_-_-

Jacksonville---_-_--_-_-----

Jacksonville Beach--_-_-_-_-

Jacksonville Suburban Utility

Jan Phy1 Village-_-_-_-_-_-

Jennings--_-_..-_-_-_-_--_

Keaton Beach-_-_-_-_-_-

Lake Alfred--_-_-_-_-_-_-_-_-_

Lake City--_-_-_-_-_-_--

Lee County Water System------

Longwood--_-_-_-_-_-_---_-

Lynn Haven-_-

Manalapan---_-_-_-_-_-_-_----

Manatee County Water System--

Marco Island-_-_-_-_-_---

Melbourne--_-_-_-_-_-_---

Mexico Beach--_-_-_-_-_--

Mims---_-_-_-_-_-_-_-_-_-

Mt. Dora--

Mulberry-_-_-_-_-_-_-_-_-_-_-_

Navarre Beach-_-_-_-_-_-_-_-

Neptune Beach--_-_-_-_-_---_

New Smyrna Beach-_-_-_-_-_-

North Miami-_-_-_-_-_-_-_-_-_

North Port Charlotte---_-----

North Port St. Lucie--_-_-_-

Okeechobee-----_----_------

Orange Park (Meadowbrook) - - -

Pace--

Pahokee---------------------

Palm Beach - Villa Del Ray---

Palm Coast-_-_-_-_-

86

87

88

89

90

91

92

93

94

95

96

97

98

99

100

101

102

103

104

105

106

107

108

109

110

111

112

113

114

115

116

117

118

119

120

121

122

123

124

125

126

127

128

129

130

131

Port Richey-_-_-_-_-_.- 132

Port St. Joe-_-_-_-_-_-_ 133 
Punta Gorda-_-_-_-_-_-_ 134 Quincy---D-D-D-D 135

Rex Utilities (Florida City) - 136 Ridge Manor---_-_-_--- 137

Ridge Utilities-_-_-_-_--.- 138

Rotonda West--_-_-_-_-- 139

Ruskin---_--_-_-_----- 140

St. Augustine-_- 141

St. Cloud-_-_-_-_-_-_ 142

St. Marks---- 143

San Antonio-_-_-_-_-_-_-_--- 144

Sarasota-_-_-_-_-_-_-_--- 145

Sawgrass-_-_-_-_-_-_-_-_-_- 146

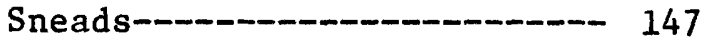

South Miami Heights--------- 148

South Walton County Utilities 149

Sunrise--_-_-_-_-_-_-_ 150

Tamarac--_-_-_-_-_-_--- 151
Tampa---- 152

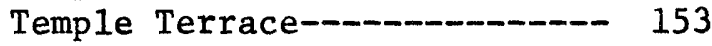

Umatilla-_-_-_-_-_-_-_ 154

Union Correctional Institute- 155

Venice---_-_-_-_-_-_-_-------- 156

Venice Gardens-_-_-_-_-_-_-_- 157

Vernon-_-_-__-_-_-_-_-_-_-_-_ 158

Wahneta--_-_-_-_-_-_-_-_-_ 159

Walt Disney World-_-_-_-_-_-- 160

Weeki Wachee---_-_-_------ 161

West Palm Beach-_-_-_-_-_-_--- 162

Wewahitchka-_-_-__-_-_-_-_ 163

White Springs-_-_-_- 164

Wildwood-_-_-_-_-_- 165

Williston-_-_____-_-_-_ 166

Woodville-_-_-_-_-_-_-- 167

Zephyrhills-_-_-_-_-_-_- 168

Zolfo Springs-_-_-_-_-_-_-_ 169 
PUBLIC WATER SUPPLY: Anastasia Sanitary District

COUNTY: St. Johns

COLLECTION DATE: 12-12-77

SAMPLING POINT (1) Treated water -295132081161001 , tap in rear of water treatment plant office.

(2) Raw water-

ANALYSIS OF MAJOR CHEMICAL CONSTITUENTS AND PHYSICAL PROPERTIES (Dissolved concentrations in milligrams per liter, except as indicated)

(1)

$\begin{array}{lc}\text { Silica (SiO2) } & 19 \\ \text { Calcium (Ca) } & 80 \\ \text { Magnesium (Mg) } & 37 \\ \text { Sodium (Na) } & 120 \\ \text { Potassium (K) } & 4.3 \\ \text { Bicarbonate (HCO3) } & 68 \\ \text { Carbonate (CO3) } & 0 \\ \text { Sulfate (SO4) } & 240 \\ \text { Chloride (C1) } & 220 \\ \text { Fluoride (F) } & .6 \\ \text { Nitrate (NO3-N), } & \\ \quad \text { total } & .12 \\ \text { Nitrite (NO2-N), } & \\ \quad \text { total } & .00\end{array}$

(2)

$$
\text { Dissolved solids }
$$$$
\text { (residue at } 180^{\circ} \mathrm{C} \text { ) } \quad 750
$$$$
\text { Hardness as } \mathrm{CaCO} 3
$$$$
(\mathrm{Ca}, \mathrm{Mg}) \quad 360
$$

Noncarbonate hardness as $\mathrm{CaCO} 3$

300

Percent sodium 42

Alkalinity as $\mathrm{CaCO} 3 \quad 56$

Specific conductance

(umhos $/ \mathrm{cm}$ at $25^{\circ} \mathrm{C}$ ) 1300

$\mathrm{pH}$ (units)

Temperature $\left({ }^{\circ} \mathrm{C}\right)$

Color (Pt-Co units)

Turbidity (NTU)
(1)

ANALYSIS OF SELECTED TRACE ELEMENTS

(Total concentrations in micrograms per liter, except as indicated)

$\begin{array}{lrlr}\text { Arsenic (As) } & 2 & \text { Manganese (Mn) } & 10 \\ \text { Barium (Ba) } & -- & \text { Mercury (Hg) } & <.5 \\ \text { Cadmium (Cd) } & 0 & \text { Selenium (Se) } & 0 \\ \text { Chromium (Cr) } & <10 & \text { Silver (Ag) } & 1 \\ \text { Copper (Cu) } & 11 & \text { Strontium, dissolved ( } \mathrm{Sr} \text { ) } 3000 \\ \text { Iron (Fe) } & 50 & \text { Zinc ( } \mathrm{nn}) & 50 \\ \text { Lead (Pb) } & 5 & & \end{array}$

ANALYSIS OF SELECTED PESTICIDE AND INDUSTRIAL COMPOUNDS

(Total concentrations in micrograms per liter)

$\begin{array}{llll}\text { Aldrin } & .00 & \text { Lindane } & .00 \\ \text { Chlordane } & .0 & \text { Methoxychlor } & .00 \\ \text { DDD } & .00 & \text { Mirex } & .00 \\ \text { DDE } & .00 & \text { PCB } & .0 \\ \text { DDT } & .00 & \text { PCN } & .00 \\ \text { Dieldrin } & .00 & \text { Silvex } & .00 \\ \text { Endrin } & .00 & \text { Toxaphene } & 0 \\ \text { Heptachlor } & .00 & 2,4-D & .00 \\ \text { Heptachlor epoxide } & .00 & 2,4,5-T & .00\end{array}$

ANALYSIS OF SELECTED GROSS RADIOACTIVITY

(Total activity concentrations in picocuries per liter)

Gross Alpha, as uranium natural $<6.3$

Gross Beta, as

cesium-137
Gross Beta, as

strontium-90
8.6

19.2

0

1 
PUBLIC WATER SUPPLY: Apalachicola COUNTY: Franklin COLLECTION DATE: 11-30-77

SAMPLING POINT (1) Treated water-294339084591990, tap outside on southeast wall of library, Gorrie Square, Apalachicola.

(2) Raw water-

ANALYSIS OF MAJOR CHEMICAL CONSTITUENTS AND PHYSICAL PROPERTIES (Dissolved concentrations in milligrams per liter, except as indicated)

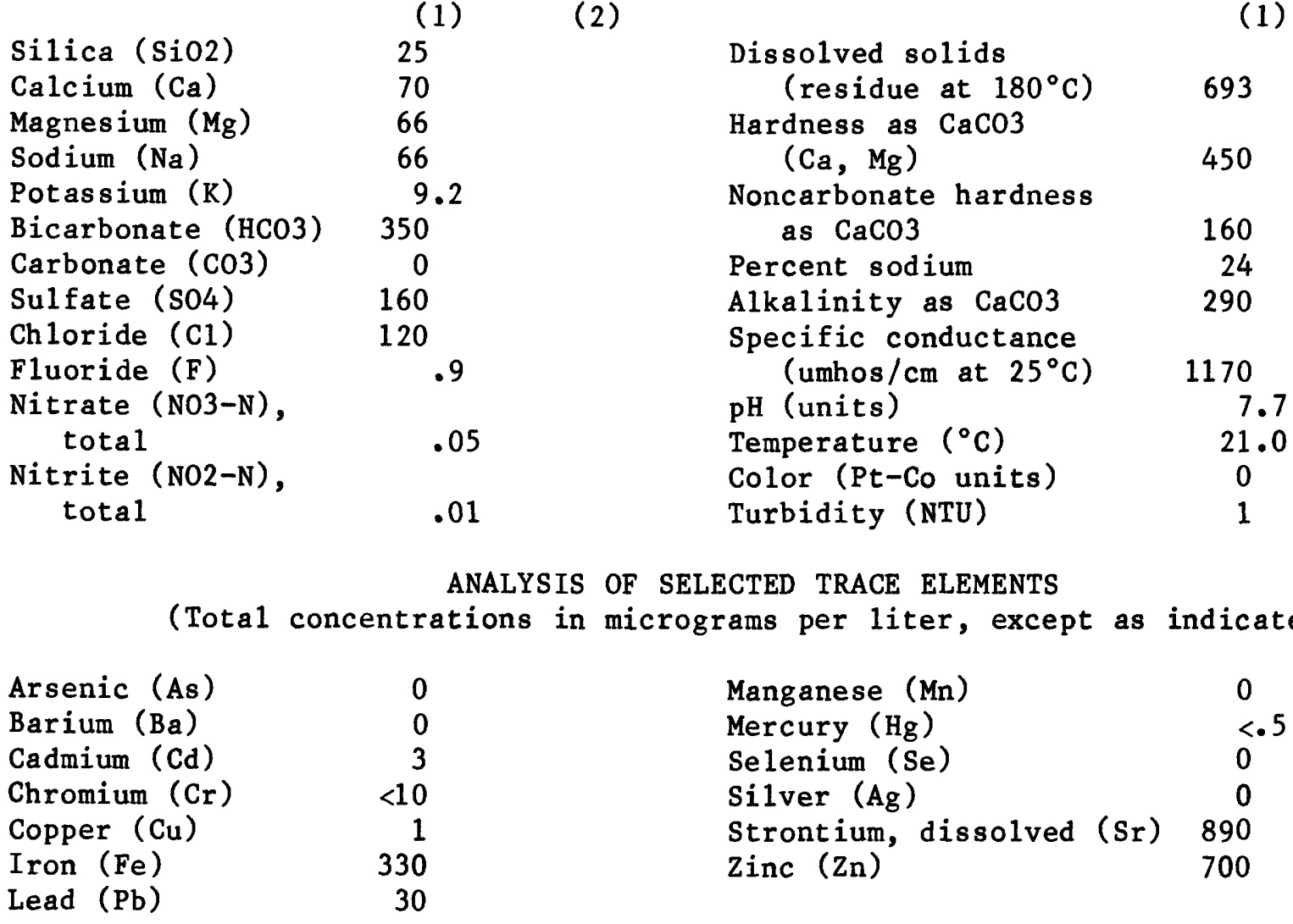

ANALYSIS OF SELECTED PESTICIDE AND INDUSTRIAL COMPOUNDS (Total concentrations in micrograms per liter)

$\begin{array}{llll}\text { Aldrin } & .00 & \text { Lindane } & .00 \\ \text { Chlordane } & .0 & \text { Methoxych1or } & .00 \\ \text { DDD } & .00 & \text { Mirex } & .00 \\ \text { DDE } & .00 & \text { PCB } & .0 \\ \text { DDT } & .00 & \text { PCN } & .00 \\ \text { Dieldrin } & .00 & \text { Silvex } & .00 \\ \text { Endrin } & .00 & \text { Toxaphene } & 0 \\ \text { Heptachlor } & .00 & 2,4-D & .00 \\ \text { Heptachlor epoxide } & .00 & 2,4,5-T & .00\end{array}$

ANALYSIS OF SELECTED GROSS RADIOACTIVITY

(Total activity concentrations in picocuries per liter)

Gross Alpha, as uranium natural 19

Gross Beta, as cesium-137
Gross Beta, as

strontium-90 10 
PUBLIC WATER SUPPLY: Arcadia

COUNTY: DeSoto

COLLECTION DATE: 12-21-77

SAMPLING POINT (1) Treated water-271253081502909, 5 well composite, tap inside water treatment plant.

(2) Raw water-

ANALYSIS OF MAJOR CHEMICAL CONSTITUENTS AND PHYSICAL PROPERTIES

(Dissolved concentrations in milligrams per liter, except as indicated)

Silica ( $\mathrm{SiO2)}$

(1)

(2)
Dissolved solids

(residue at $180^{\circ} \mathrm{C}$ )

Hardness as $\mathrm{CaCO} 3$

$(\mathrm{Ca}, \mathrm{Mg})$

Noncarbonate hardness

as $\mathrm{CaCO} 3$

Percent sodium

Alkalinity as $\mathrm{CaCO} 3$

Specific conductance

(umhos $/ \mathrm{cm}$ at $25^{\circ} \mathrm{C}$ )

$\mathrm{pH}$ (units)

Temperature $\left({ }^{\circ} \mathrm{C}\right)$

Color (Pt-Co units)

Turbidity (NTU)

(1)

ANALYSIS OF SELECTED TRACE ELEMENTS

(Total concentrations in micrograms per liter, except as indicated)

Arsenic (As)

Barium ( $\mathrm{Ba}$ )

Cadmium (Cd)

Chromium ( $\mathrm{Cr}$ )

Copper (Cu)

Iron ( $\mathrm{Fe}$ )

Lead $(\mathrm{Pb})$
Manganese (Mn)

Mercury ( $\mathrm{Hg}$ )

Selenium ( $\mathrm{Se}$ )

Silver ( $\mathrm{Ag})$

Strontium, dissolved (Sr)

Zinc $(\mathrm{Zn})$

ANALYSIS OF SELECTED PESTICIDE AND INDUSTRIAL COMPOUNDS (Total concentrations in micrograms per 1iter)

$\begin{array}{ll}\text { Aldrin } & \text { Lindane } \\ \text { Ch lordane } & \text { Methoxychlor } \\ \text { DDD } & \text { Mirex } \\ \text { DDE } & \text { PCB } \\ \text { DDT } & \text { PCN } \\ \text { Dieldrin } & \text { Silvex } \\ \text { Endrin } & \text { Toxaphene } \\ \text { Heptachlor } & 2,4-D \\ \text { Heptachlor epoxide } & 2,4,5-\mathrm{T}\end{array}$

ANALYSIS OF SELECTED GROSS RADIOACTIVITY

(Total activity concentrations in picocuries per liter)

Gross Alpha, as uranium natural

Gross Beta, as

cesium-137
8.2

Gross Beta, as

strontium -90 
PUBLIC WATER SUPPLY: Atlantic Beach

COUNTY: Duva1

COLLECTION DATE: $12-13-77$

SAMPLING POINT (1) Treated water -302007081242101 , tap on corner of 11 th Street water treatment plant.

(2) Raw water-

ANALYSIS OF MAJOR CHEMICAL CONSTITUENTS AND PHYSICAL PROPERTIES

(Dissolved concentrations in milligrams per liter, except as indicated)

Silica (SiO2)

Calcium ( $\mathrm{Ca}$ )

Magnesium ( $\mathrm{Mg})$

Sodium $(\mathrm{Na})$

Potassium ( $\mathrm{K}$ )

Bicarbonate ( $\mathrm{HCO3}$ )

Carbonate ( $\mathrm{CO} 3)$

Sulfate (S04)

Chloride (C1)

Fluoride (F)

Nitrate $(\mathrm{NO} 3-\mathrm{N})$, total

Nitrite (NO2-N), total
(2)

(1)

$$
\begin{aligned}
& \text { Dissolved solids } \\
& \text { (residue at } 180^{\circ} \mathrm{C} \text { ) } \\
& \text { Hardness as CaCO3 } \\
& \text { (Ca, Mg) } \\
& \text { Noncarbonate hardness } \\
& \text { as CaC03 } \\
& \text { Percent sodium } \\
& \text { Alkalinity as CaC03 } \\
& \text { Specific conductance } \\
& \quad \text { (umhos/cm at } 25^{\circ} \mathrm{C} \text { ) } \\
& \text { pH (units) } \\
& \text { Temperature ( }{ }^{\circ} \mathrm{C} \text { ) } \\
& \text { Color (Pt-Co units) } \\
& \text { Turbidity (NTU) }
\end{aligned}
$$

ANALYSIS OF SELECTED TRACE ELEMENTS

(Total concentrations in micrograms per liter, except as indicated)

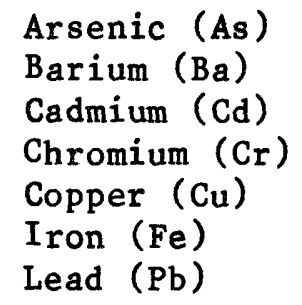

Manganese (Mn)

Mercury ( $\mathrm{Hg}$ )

Selenium ( $\mathrm{Se}$ )

Silver $(\mathrm{Ag})$

Strontium, dissolved ( $\mathrm{Sr}$ )

Zinc $(\mathrm{Zn})$

ANALYSIS OF SELECTED PESTICIDE AND INDUSTRIAL COMPOUNDS

(Total concentrations in micrograms per liter)

Aldrin
Chlordane
DDD
DDE
DDT
Dieldrin
Endrin
Heptachlor
Heptachlor epoxide

Aldrin

Chlordane

DDD

DDE

Dieldrin

Heptachlor

Heptachlor epoxide
Lind ane

Methoxych 1or

Mirex

PCB

PCN

Silvex

Toxaphene

2,4-D

$2,4,5-\mathrm{T}$
720

7.2

24.5

(1) 
PUBLIC WATER SUPPLY: Auburn

COUNTY: Okaloosa

COLLECTION DATE: 01-04-78

SAMPLING POINT (1) Treated water-304800086320090, tap on west side of Auburn Water System Inc. building.

(2) Raw water-

ANALYSIS OF MAJOR CHEMICAL CONSTITUENTS AND PHYSICAL PROPERTIES (Dissolved concentrations in milligrams per liter, except as indicated)

(1)

$\begin{array}{lc}\text { Silica (SiO2) } & 11 \\ \text { Calcium (Ca) } & 26 \\ \text { Magnesium (Mg) } & 12 \\ \text { Sodium (Na) } & 4.6 \\ \text { Potassium (K) } & 1.0 \\ \text { Bicarbonate (HCO3) } & 120 \\ \text { Carbonate (CO3) } & 0 \\ \text { Sulfate (SO4) } & 7.9 \\ \text { Chloride (C1) } & 5.9 \\ \text { Fluoride (F) } & .1 \\ \text { Nitrate (NO3-N), } & \\ \quad \text { total (NO2-N), } & .00 \\ \text { Nitrite (NO2-N), } & \\ \quad \text { total } & .00\end{array}$

(2)

$\begin{array}{lc}\text { Dissolved solids } & (1) \\ \quad \text { (residue at } 180^{\circ} \mathrm{C} \text { ) } & 132 \\ \text { Hardness as CaCO3 } & \\ \quad \text { (Ca, Mg) } & 110 \\ \text { Noncarbonate hardness } & \\ \quad \text { as CaCO3 } & 16 \\ \text { Percent sodium } & 8 \\ \text { Alkalinity as CaCO3 } & 98 \\ \text { Specific conductance } & \\ \quad \text { (umhos/cm at } 25^{\circ} \mathrm{C} \text { ) } & 200 \\ \text { pH (units) } & 7.9 \\ \text { Temperature ( }{ }^{\circ} \mathrm{C} \text { ) } & 10.0 \\ \text { Color (Pt-Co units) } & 4 \\ \text { Turbidity (NTU) } & 1\end{array}$

ANALYSIS OF SELECTED TRACE ELEMENTS

(Total concentrations in micrograms per liter, except as indicated)

$\begin{array}{lrlc}\text { Arsenic (As) } & 10 & \text { Manganese (Mn) } & 0 \\ \text { Barium (Ba) } & - & \text { Mercury (Hg) } & .6 \\ \text { Cadmium (Cd) } & 0 & \text { Selenium (Se) } & 0 \\ \text { Chromium (Cr) } & <10 & \text { Silver (Ag) } & 0 \\ \text { Copper (Cu) } & 43 & \text { Strontium, dissolved ( Sr) } & 60 \\ \text { Iron (Fe) } & 90 & \text { Zinc (Zn) } & 10 \\ \text { Lead (Pb) } & 27 & & \end{array}$

ANALYSIS OF SELECTED PESTICIDE AND INDUSTRIAL COMPOUNDS (Total concentrations in micrograms per liter)

$\begin{array}{llll}\text { Aldrin } & .00 & \text { Lindane } & .00 \\ \text { Chlordane } & .0 & \text { Methoxychlor } & .00 \\ \text { DDD } & .00 & \text { Mirex } & .00 \\ \text { DDE } & .00 & \text { PCB } & .0 \\ \text { DDT } & .00 & \text { PCN } & .00 \\ \text { Dieldrin } & .00 & \text { Silvex } & .00 \\ \text { Endrin } & .00 & \text { Toxaphene } & 0 \\ \text { Heptachlor } & .00 & 2,4-D & .00 \\ \text { Heptachlor epoxide } & .00 & 2,4,5-T & .00\end{array}$

ANALYSIS OF SELECTED GROSS RADIOACTIVITY

(Total activity concentrations in picocuries per liter)

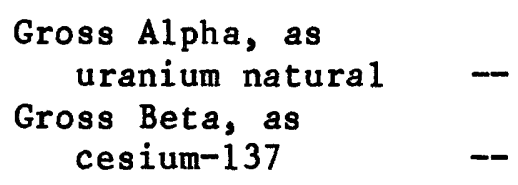

Gross Beta, as

strontium-90 
PUBLIC WATER SUPPLY: Avon Park

COUNTY: Highlands

COLLECTION DATE: 11-10-77

SAMPLING POINT (1) Treated water -273527081310801 , tap near Be11 Street well.

(2) Raw water-

ANALYSIS OF MAJOR CHEMICAL CONSTITUENTS AND PHYSICAL PROPERTIES (Dissolved concentrations in milligrams per liter, except as indicated)

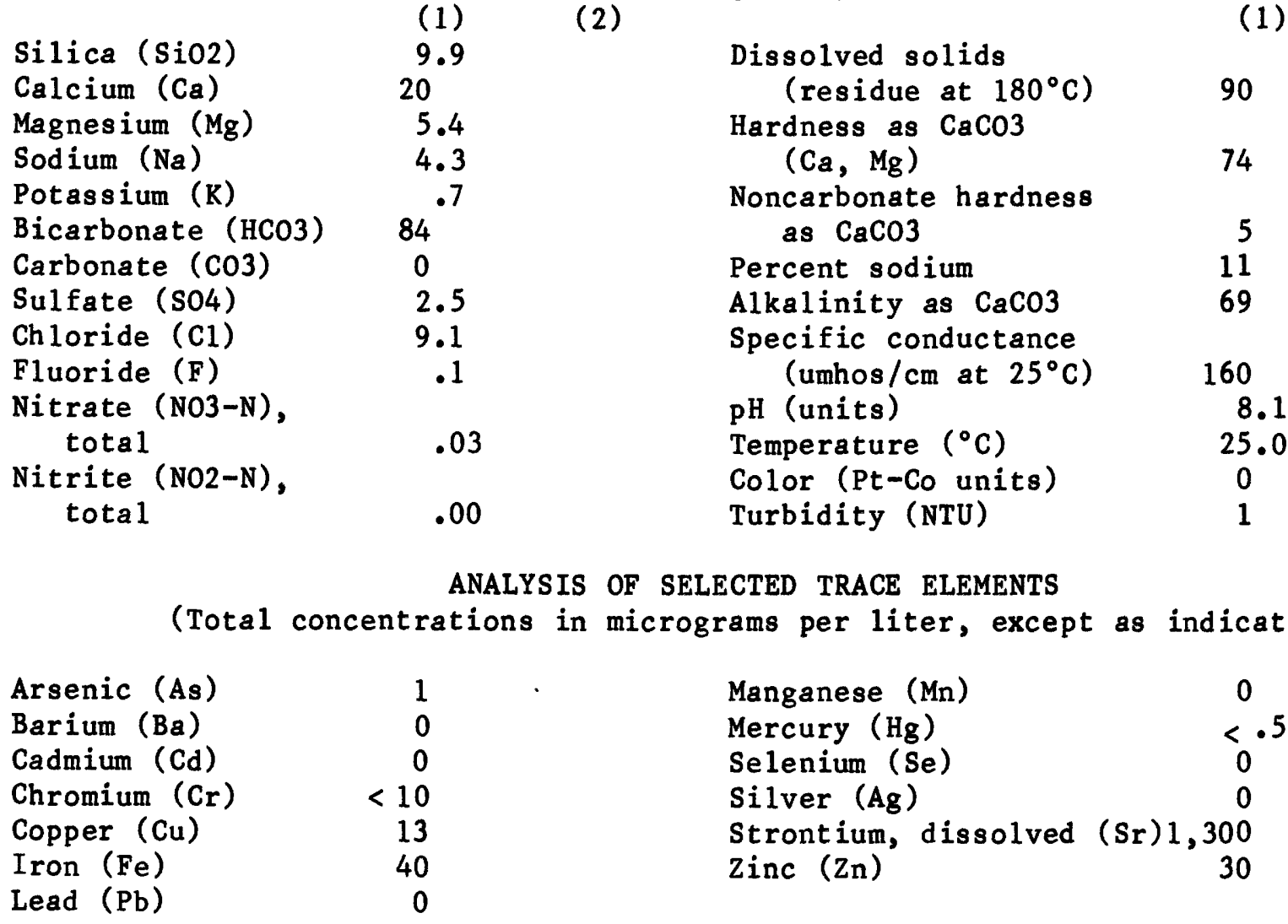

ANALYSIS OF SELECTED PESTICIDE AND INDUSTRIAL COMPOUNDS

(Total concentrations in micrograms per liter)

$\begin{array}{lllr}\text { Aldrin } & .00 & \text { Lindane } & .00 \\ \text { Chlordane } & .0 & \text { Methoxych lor } & .00 \\ \text { DDD } & .00 & \text { Mirex } & -- \\ \text { DDE } & .00 & \text { PCB } & .0 \\ \text { DDT } & .00 & \text { PCN } & .00 \\ \text { Dieldrin } & .00 & \text { Silvex } & .00 \\ \text { Endrin } & .00 & \text { Toxaphene } & 0 \\ \text { Heptachlor } & .00 & 2,4-D & .00 \\ \text { Heptachlor epoxide } & .00 & 2,4,5-T & .00\end{array}$

ANALYSIS OF SELECTED GROSS RADIOACTIVITY

(Total activity concentrations in picocuries per liter)

Gross Alpha, as

uranium natural $\quad 5.6$

Gross Beta, as

cesium-137
Gross Beta, as

strontium -90

$<.4$ 
PUBLIC WATER SUPPLY: Baker COUNTY: Okaloosa COLLECTION DATE: 01-04-78

SAMPLING POINT (1) Treated water-304801086403190, tap under holding tank in rear of water treatment building (east side).

(2) Raw water-

ANALYSIS OF MAJOR CHEMICAL CONSTITUENTS AND PHYSICAL PROPERTIES (Dissolved concentrations in milligrams per liter, except as indicated)
(1)
(2)
(1)

$\begin{array}{lc}\text { Silica (SiO2) } & 21 \\ \text { Calcium ( } \mathrm{Ca}) & 10 \\ \text { Magnesium (Mg) } & 6.3 \\ \text { Sodium (Na) } & 60 \\ \text { Potassium (K) } & 6.0 \\ \text { Bicarbonate (HCO3) } & 170 \\ \text { Carbonate (CO3) } & 0 \\ \text { Sulfate (SO4) } & 6.3 \\ \text { Chloride (C1) } & 20 \\ \text { Fluoride (F) } & .6 \\ \text { Nitrate (NO3-N), } & \\ \quad \text { total (NO2-N), } \\ \text { Nitrite } \\ \quad \text { total }\end{array}$

Dissolved solids

(residue at $180^{\circ} \mathrm{C}$ )

215

Hardness as $\mathrm{CaCO} 3$

$(\mathrm{Ca}, \mathrm{Mg})$

51

Noncarbonate hardness as $\mathrm{CaCO} 3$

Percent sodium

Alkalinity as $\mathrm{CaCO} 3$

Specific conductance (umhos $/ \mathrm{cm}$ at $25^{\circ} \mathrm{C}$ )

$\mathrm{pH}$ (units)

Temperature $\left({ }^{\circ} \mathrm{C}\right)$

Color (Pt-Co units)

Turbidity (NTU)

ANALYSIS OF SELECTED TRACE ELEMENTS

(Total concentrations in micrograms per liter, except as indicated)

$\begin{array}{lrlr}\text { Arsenic (As) } & 1 & \text { Manganese (Mn) } & 0 \\ \text { Barium (Ba) } & - & \text { Mercury (Hg) } & <.5 \\ \text { Cadmium ( } \mathrm{Cd}) & 0 & \text { Selenium (Se) } & 0 \\ \text { Chromium (Cr) } & <10 & \text { Silver (Ag) } & 0 \\ \text { Copper (Cu) } & 5 & \text { Strontium, dissolved (Sr) } & 360 \\ \text { Iron ( } \mathrm{Fe}) & 40 & \text { Zinc (Zn) } & 10 \\ \text { Lead ( } \mathrm{Pb}) & 6 & & \end{array}$

ANALYSIS OF SELECTED PESTICIDE AND INDUSTRIAL COMPOUNDS ( Total concentrations in micrograms per liter)

$\begin{array}{llll}\text { Aldrin } & .00 & \text { Lindane } & .00 \\ \text { Chlordane } & .0 & \text { Methoxychlor } & .00 \\ \text { DDD } & .00 & \text { Mirex } & .00 \\ \text { DDE } & .00 & \text { PCB } & .0 \\ \text { DDT } & .00 & \text { PCN } & .00 \\ \text { Dieldrin } & .00 & \text { Silvex } & .00 \\ \text { Endrin } & .00 & \text { Toxaphene } & 0 \\ \text { Heptachlor } & .00 & 2,4-D & .00 \\ \text { Heptachlor epoxide } & .00 & 2,4,5-T & .00\end{array}$

ANALYSIS OF SELECTED GROSS RADIOACTIVITY

(Total activity concentrations in picocuries per liter)

Gross Alpha, as uranium natural 1.8 Gross Beta, as cesium-137
Gross Beta, as

strontium-90

5.8 
PUBLIC WATER SUPPLY: Bay County Water System

COUNTY: Bay

COLLECTION DATE: 12-22-77

SAMPLING POINT (1) Treated water-301215085370590, Tyndal1 Air Force Base, Wherry II bousing area, 2445 Lincoln Drive, outside tap.

(2) Raw water-301215085370500; (Deer Doint lake) tap in second floor water treatment laboratory.

ANALYSIS OF MAJOR CHEMICAL CONSTITUENTS AND PHYSICAL PROPERTIES

(Dissolved concentrations in milligrams per liter, except as indicated)

Silica (Si02)

Calcium ( $\mathrm{Ca}$ )

Magnesium $(\mathrm{Mg})$

Sodium ( $\mathrm{Na})$

Potassium ( $\mathrm{K}$ )

Bicarbonate ( $\mathrm{HCO} 3$ )

Carbonate ( $\mathrm{CO} 3)$

Sulfate (s04)

Chloride (C1)

Fluoride (F)

Nitrate (NO3-N),

total

Nitrite $(\mathrm{NO2}-\mathrm{N})$,

total
(1)

\section{0}

25

2.0

4.3

0

11

14

.6

$.01 \quad .00$

$.00 \quad .00$
$40^{.5}$
(2)

\section{7}

10

1.8

4.4

.5

33

0

3.0

9.5

.0
Dissolved solids

(residue at $180^{\circ} \mathrm{C}$ )

Hardness as $\mathrm{CaCO}$.

$(\mathrm{Ca}, \mathrm{Mg}$ )

Noncarbonate hardness as $\mathrm{CaCO} 3$

Percent sodium

Alkalinity as $\mathrm{CaCO} 3$

Specific conductance

(umhos $/ \mathrm{cm}$ at $25^{\circ} \mathrm{C}$ )

$\mathrm{pH}$ (units)

Temperature $\left({ }^{\circ} \mathrm{C}\right)$

Color (Pt-Co units)

Turbidity (NTU)
(1)

(2)

93

54

58

33

25

14

33

22

ANALYSIS OF SELECTED TRACE ELEMENTS

(Total concentrations in micrograms per liter, except as indicated)
Arsenic (As)

Barium ( $\mathrm{Ba}$ )

Cadmium ( $C d)$

Chromium ( $\mathrm{Cr}$ )

Copper $(\mathrm{Cu})$

Iron ( $\mathrm{Fe}$ )

Lead $(\mathrm{Pb})$

$\begin{array}{rr}3 & 0 \\ -- & - \\ 0 & 0 \\ <10 & 10 \\ 18 & 2 \\ 200 & 170 \\ 44 & 21\end{array}$

ANALYSIS OF SELECTED PESTICIDE AND INDUSTRIAL COMPOUNDS

(Total concentrations in micrograms per liter)

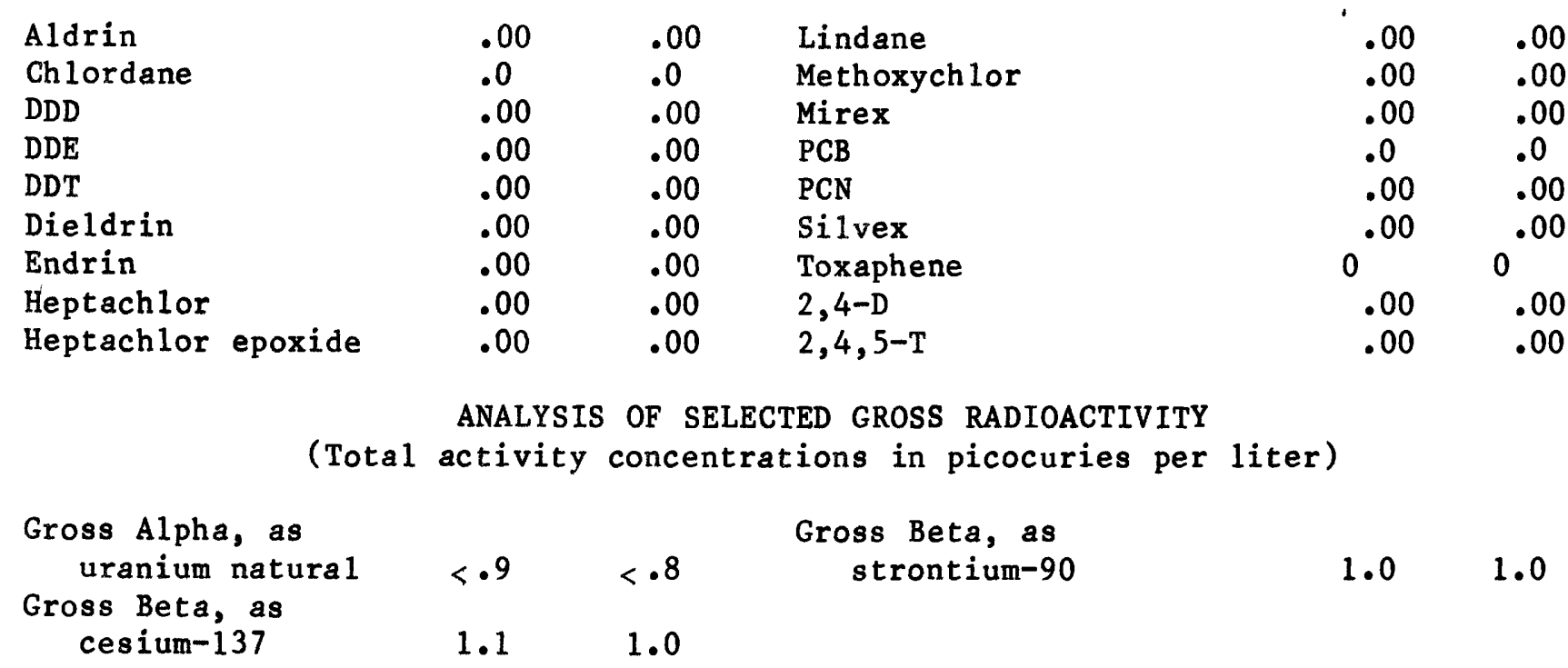


PUBLIC WATER SUPPLY: Bellair

COUNTY: Pinellas

COLLECTION DATE: 12-20-77

SAMPLING POINT (1) Treated water-275603082480409, tap inside pumphouse at northwest end of building.

(2) Raw water-

ANALYSIS OF MAJOR CHEMICAL CONSTITUENTS AND PHYSICAL PROPERTIES (Dissolved concentrations in milligrams per liter, except as indicated)

(1) (2)

\begin{tabular}{lc} 
Silica (SiO2) & 26 \\
Calcium (Ca) & 56 \\
Magnesium (Mg) & 7.4 \\
Sodium (Na) & 16 \\
Potassium (K) & 1.1 \\
Bicarbonate (HCO3) & 160 \\
Carbonate (CO3) & 0 \\
Sulfate (SO4) & 6.7 \\
Chloride (C1) & \multicolumn{2}{c}{45} \\
Fluoride (F) & .2 \\
Nitrate (NO3-N), & \\
$\quad$ total (NO2-N), & .02 \\
Nitrite (NO2-N) \\
$\quad$ total
\end{tabular}

(1)

Dissolved solids

(residue at $180^{\circ} \mathrm{C}$ ) 241

Hardness as $\mathrm{CaCO} 3$ $(\mathrm{Ca}, \mathrm{Mg})$

170

Noncarbonate hardness as $\mathrm{CaCO} 3$ 39

Percent sodium 17

Alkalinity as $\mathrm{CaCO} 3 \quad 130$

Specific conductance

(umhos $/ \mathrm{cm}$ at $25^{\circ} \mathrm{C}$ )

$\mathrm{pH}$ (units)

Temperature $\left({ }^{\circ} \mathrm{C}\right)$

Color (Pt-Co units)

Turbidity (NTU)

ANALYSIS OF SELECTED TRACE ELEMENTS

(Total concentrations in micrograms per liter, except as indicated)

$\begin{array}{lrlc}\text { Arsenic (As) } & 3 & \text { Manganese (Mn) } & 10 \\ \text { Barium (Ba) } & - & \text { Mercury (Hg) } & <.5 \\ \text { Cadmium (Cd) } & 0 & \text { Selenium ( } \mathrm{Se}) & 0 \\ \text { Chromium (Cr) } & <10 & \text { Silver (Ag) } & 2 \\ \text { Copper (Cu) } & 3 & \text { Strontium, dissolved ( } \mathrm{Sr} \text { ) } & 120 \\ \text { Iron ( } \mathrm{Fe}) & 40 & \text { Zinc ( } \mathrm{n} \text { ) } & 30 \\ \text { Lead (Pb) } & 1 & & \end{array}$

ANALYSIS OF SELECTED PESTICIDE AND INDUSTRIAL COMPOUNDS

(Total concentrations in micrograms per liter)

$\begin{array}{llll}\text { Aldrin } & .00 & \text { Lindane } & .00 \\ \text { Chlordane } & .0 & \text { Methoxychlor } & .00 \\ \text { DDD } & .00 & \text { Mirex } & .00 \\ \text { DDE } & .00 & \text { PCB } & .0 \\ \text { DDT } & .00 & \text { PCN } & .00 \\ \text { Dieldrin } & .00 & \text { Silvex } & .00 \\ \text { Endrin } & .00 & \text { Toxaphene } & 0 \\ \text { Heptachlor } & .00 & 2,4-D & .00 \\ \text { Heptachlor epoxide } & .00 & 2,4,5-T & .00\end{array}$

ANALYSIS OF SELECTED GROSS RADIOACTIVITY

(Total activity concentrations in picocuries per liter)

Gross Alpha, as uranium natural 2.0

Gross Beta, as

cesium-137
Gross Beta, as

strontium-90

1.0 
PUBLIC WATER SUPPLY: Belle Glade COUNTY: Palm Beach

COLLECTION DATE: 12-06-77

SAMPLING POINT (1) Treated water-264125080404302, Belle Glade water treatment plant 1aboratory.

(2) Raw water-264125080404300, Belle Glade water treatment plant intake, tap in front yard, northwest corner.

ANALYSIS OF MAJOR CHEMICAL CONSTITUENTS AND PHYSICAL PROPERTIES

(Dissolved concentrations in milligrams per liter, except as indicated)

(1) (2) (1)

\begin{tabular}{|c|c|c|c|c|c|}
\hline Silica $\left(\mathrm{SiO}_{2}\right)$ & 9.0 & 16 & Dissolved solids & & \\
\hline Calcium $(\mathrm{Ca})^{-}$ & 59 & 73 & (residue at $180^{\circ} \mathrm{C}$ ) & 489 & 632 \\
\hline Magnesium (Mg) & 7.3 & 32 & Hardness as $\mathrm{CaCO}_{3}$ & & \\
\hline Sodium ( $\mathrm{Na})$ & 80 & 95 & $(\mathrm{Ca}, \mathrm{Mg})$ & 180 & 320 \\
\hline Potassium (K) & 5.8 & 5.8 & Noncarbonate hardness & & \\
\hline Bicarbonate $\left(\mathrm{HCO}_{3}\right)$ & 52 & 276 & as $\mathrm{CaCO}_{3}$ & 120 & 89 \\
\hline Carbonate $\left(\mathrm{CO}_{3}\right)$ & 10 & 0 & Percent sodium & 49 & 39 \\
\hline Sulfate $\left(\mathrm{SO}_{4}\right)$ & 120 & 94 & Alkalinity as $\mathrm{CaCO}_{3}$ & 59 & 320 \\
\hline Chloride (C1) & 140 & 150 & Specific conductance & & \\
\hline Fluoride (F) & .4 & .5 & (umhos $/ \mathrm{cm}$ at $25^{\circ} \mathrm{C}$ ) & 750 & 950 \\
\hline Nitrate $\left(\mathrm{NO}_{3}-\mathrm{N}\right)$, & & & $\mathrm{pH}$ (units) & 9.7 & 7.9 \\
\hline total & .21 & .16 & Temperature $\left({ }^{\circ} \mathrm{C}\right)$ & 25.0 & 24.5 \\
\hline Nitrite $\left(\mathrm{NO}_{2}-\mathrm{N}\right)$, & & & Color (Pt-Co units) & 0 & 70 \\
\hline total & .00 & .01 & Turbidity (NTJ) & 1 & 1 \\
\hline (Total c & $\begin{aligned} & \text { AN } \\
& \text { atic }\end{aligned}$ & $\begin{array}{l}\text { YSIS OF } \\
\text { s in } \mathrm{mi}\end{array}$ & $\begin{array}{l}\text { ECTED TRACE ELEMENTS } \\
\text { rams per liter, except }\end{array}$ & indica & \\
\hline Arsenic (As) & 1 & 3 & Manganese $(M n)$ & 0 & 10 \\
\hline Barium ( $\mathrm{Ba})$ & 0 & 100 & Mercury (Hg) & $<.5$ & $<.5$ \\
\hline Cadmium (Cd) & 0 & 1 & Selenium (Se) & 0 & 0 \\
\hline Chromium (Cr) & 10 & $<10$ & Silver $(\mathrm{Ag})$ & 0 & 0 \\
\hline Copper ( $\mathrm{Cu})$ & 3 & 3 & Strontium, dissolved $(\mathrm{Sr})$ & 1000 & 1500 \\
\hline Iron $(\mathrm{Fe})$ & 30 & 20 & Zinc $(Z n)$ & 0 & 20 \\
\hline Lead $(\mathrm{Pb})$ & 6 & 13 & & & \\
\hline
\end{tabular}

ANALYSIS OF SELECTED PESTICIDE AND INDUSTRIAL COMPOUNDS (Total concentrations in micrograms per liter)

$\begin{array}{llllll}\text { Aldrin } & .00 & .00 & \text { Lindane } & .00 & .00 \\ \text { Chlordane } & .0 & .0 & \text { Methoxych1or } & .00 & .00 \\ \text { DDD } & .00 & .00 & \text { Mirex } & .00 & .00 \\ \text { DDE } & .00 & .00 & \text { PCB } & .0 & .0 \\ \text { DDT } & .00 & .00 & \text { PCN } & .00 & .00 \\ \text { Dieldrin } & .00 & .00 & \text { Silvex } & .00 & .00 \\ \text { Endrin } & .00 & .00 & \text { Toxaphene } & 0 & 0 \\ \text { Heptachlor } & .00 & .00 & 2,4-D & .00 & .04 \\ \text { Heptachlor epoxide } & .00 & .00 & 2,4,5-T & .00 & .00\end{array}$

ANALYSIS OF SELECTED GROSS RADIOACTIVITY

(Total activity concentrations in picocuries per liter)

Gross Alpha, as

uranium natural $<3.7<8.2$

Gross Beta, as

cesium-137
Gross Beta, as

strontium-90
$7.3 \quad 8.5$ 
PUBLIC WATER SUPPLY: Belleview

COUNTY: Marion

COLLECTION DATE: 11-08-77

SAMPLING POINT (1) Treated water-290339082032001, tap outside pumphouse building.

(2) Raw water-

ANALYSIS OF MAJOR CHEMICAL CONSTITUENTS AND PHYSICAL PROPERTIES

(Dissolved concentrations in milligrams per liter, except as indicated)
(1)
(2)
(1)

$\begin{array}{lc}\text { Silica ( } & 12 \\ \text { Calcium (Ca) } & 63 \\ \text { Magnesium (Mg) } & 12 \\ \text { Sodium (Na) } & 8.7 \\ \text { Potassium (K) } & 1.0 \\ \text { Bicarbonate (HCO3) } & 170 \\ \text { Carbonate (CO3) } & 0 \\ \text { Sulfate (SO4) } & 70 \\ \text { Chloride (C1) } & 14 \\ \text { Fluoride (F) } & .2 \\ \text { Nitrate (NO3-N), } & \\ \quad \text { total (NO2-N), } & .36 \\ \text { Nitrite (NOtal } & \\ \quad \text { total }\end{array}$

Dissolved solids

(residue at $180^{\circ} \mathrm{C}$ ) 278

Hardness as $\mathrm{CaCO} 3$

$(\mathrm{Ca}, \mathrm{Mg}) \quad 210$

Noncarbonate hardness

as $\mathrm{CaCO} 3 \quad 68$

Percent sodium

Alkalinity as $\mathrm{CaCO} 3$

Specific conductance

(umhos $/ \mathrm{cm}$ at $25^{\circ} \mathrm{C}$ )

$\mathrm{pH}$ (units)

Temperature $\left({ }^{\circ} \mathrm{C}\right)$

Color (Pt-Co units)

Turbidity (NTU)

ANALYSIS OF SELECTED TRACE ELEMENTS

(Total concentrations in micrograms per liter, except as indicated)

$\begin{array}{lrlr}\text { Arsenic (As) } & 4 & \text { Manganese (Mn) } & 10 \\ \text { Barium (Ba) } & 0 & \text { Mercury (Hg) } & 1.8 \\ \text { Cadmium ( } \mathrm{Cd}) & 1 & \text { Selenium ( Se) } & 0 \\ \text { Chromium ( } \mathrm{Cr}) & 10 & \text { Silver (Ag) } & 0 \\ \text { Copper (Cu) } & 4 & \text { Strontium, dissolved ( Sr) } & 700 \\ \text { Iron (Fe) } & 30 & \text { Zinc (Zn) } & 20 \\ \text { Lead (Pb) } & 6 & & \end{array}$

\section{ANALYSIS OF SELECTED PESTICIDE AND INDUSTRIAL COMPOUNDS}

(Total concentrations in micrograms per 1iter)

$\begin{array}{llll}\text { Aldrin } & .00 & \text { Lindane } & .00 \\ \text { Chlordane } & .0 & \text { Methoxychlor } & .00 \\ \text { DDD } & .00 & \text { Mirex } & .00 \\ \text { DDE } & .00 & \text { PCB } & .0 \\ \text { DDT } & .00 & \text { PCN } & .00 \\ \text { Dieldrin } & .00 & \text { Silvex } & .00 \\ \text { Endrin } & .00 & \text { Toxaphene } & 0 \\ \text { Heptachlor } & .00 & 2,4-D & .00 \\ \text { Heptachlor epoxide } & .00 & 2,4,5-T & .00\end{array}$

ANALYSIS OF SELECTED GROSS RADIOACTIVITY

(Total activity concentrations in picocuries per liter)

Gross Alpha, as uranium natural $\quad 3.4$

Gross Beta, as

Cesium-137
Gross Beta, as

strontium -90

9.1 
PUBLIC WATER SUPPLY: Bradenton

\section{COUNTY: Manatee}

COLLECTION DATE: 12-21-77

SAMPLING POINT (1) Treated water-272935082335009, tap outside, behind water treatment plant.

(2) Raw water-272935082335000, tap inside water treatment plant.

ANALYSIS OF MAJOR CHEMICAL CONSTITUENTS AND PHYSICAL PROPERTIES

(Dissolved concentrations in milligrams per liter, except as indicated)
(1)
(2)
(1)

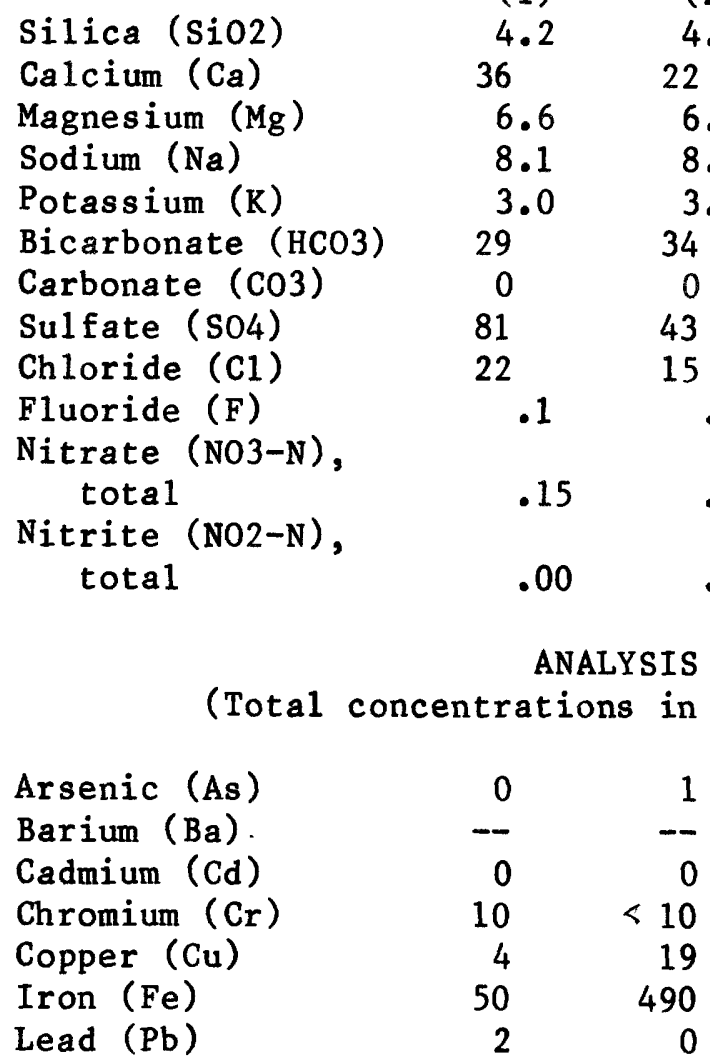

Dissolved solids

$22 \quad$ (residue at $180^{\circ} \mathrm{C}$ ) $178 \quad 153$

6.2 Hardness as $\mathrm{CaCO} 3$

$8.7 \quad(\mathrm{Ca}, \mathrm{Mg}) \quad 120$

3.1 Noncarbonate hardness

34 as $\mathrm{CaCO} 3$

Percent sodium

Alkalinity as $\mathrm{CaCO} 3$

Specific conductance

(umhos $/ \mathrm{cm}$ at $25^{\circ} \mathrm{C}$ )

$\mathrm{pH}$ (units)

.16 Temperature $\left({ }^{\circ} \mathrm{C}\right)$

Color (Pt-Co units)

.00 Turbidity (NTU)

$\begin{array}{ll}95 & 54 \\ 13 & 18 \\ 24 & 28\end{array}$

.1

.00

$\begin{array}{cc}-8.2 & -- \\ -- & -- \\ 2 & 160 \\ 1 & 1\end{array}$

ANALYSIS OF SELECTED PESTICIDE AND INDUSTRIAL COMPOUNDS

(Total concentrations in micrograms per 1 iter)

$\begin{array}{llllrr}\text { Aldrin } & .00 & .00 & \text { Lindane } & .00 & .00 \\ \text { Chlordane } & .0 & .0 & \text { Methoxych 1or } & .00 & .00 \\ \text { DDD } & .00 & .00 & \text { Mirex } & .00 & .00 \\ \text { DDE } & .00 & .00 & \text { PCB } & .0 & .0 \\ \text { DDT } & .00 & .00 & \text { PCN } & .00 & .00 \\ \text { Dieldrin } & .00 & .00 & \text { Silvex } & .00 & -- \\ \text { Endrin } & .00 & .00 & \text { Toxaphene } & 0 & 0 \\ \text { Heptachlor } & .00 & .00 & 2,4-D & .0 & -- \\ \text { Heptachlor epoxide } & .00 & .00 & 2,4,5-\mathrm{T} & .0 & --\end{array}$

ANALYSIS OF SELECTED GROSS RADIOACTIVITY

(Total activity concentrations in picocuries per liter)

Gross Alpha, as

uranium natural

Gross Beta, as

cesium-137
$2.0 \quad 1.9$

6.28 .9
Gross Beta, as

strontium-90

$5.7 \quad 7.9$ 
PUBLIC WATER SUPPLY: Branford COUNTY: Suwannee COLLECTION DATE: 11-09-77

SAMPLING POINT (1) Treated water-295734082554390, tap outside on east end of fire department/water treatment plant.

(2) Raw water-

ANALYSIS OF MAJOR CHEMICAL CONSTITUENTS AND PHYSICAL PROPERTIES

(Dissolved concentrations in milligrams per liter, except as indicated)

(1) (2) (1)

Silica (SiO2)

Calcium ( $\mathrm{Ca}$ )

Magnesium ( $\mathrm{Mg})$

Sodium ( $\mathrm{Na})$

Potassium (K)

Bicarbonate ( $\mathrm{HCO} 3$ )

Carbonate ( $\mathrm{CO} 3)$

Sulfate (s04)

Chloride (C1)

Fluoride (F)

Nitrate (NO3-N),

total

Nitrite (NO2-N),

total

\section{0}

96

39

17

3.0

260

0

200

12

.2

1.7

.00
Dissolved solids

(residue at $180^{\circ} \mathrm{C}$ )

Hardness as $\mathrm{CaCO} 3$

$(\mathrm{Ca}, \mathrm{Mg})$

545

400

Noncarbonate hardness as $\mathrm{CaCO} 3$

Percent sodium

Alkalinity as $\mathrm{CaCO} 3$

Specific conductance

(umhos $/ \mathrm{cm}$ at $25^{\circ} \mathrm{C}$ )

$\mathrm{pH}$ (units)

Temperature $\left({ }^{\circ} \mathrm{C}\right)$

Color (Pt-Co units)

Turbidity (NTU)
190

8

210

810

7.1

22.0

1

2

ANALYSIS OF SELECTED TRACE ELEMENTS

(Total concentrations in micrograms per liter, except as indicated)

$\begin{array}{lr}\text { Arsenic (As) } & 1 \\ \text { Barium (Ba) } & 0 \\ \text { Cadmium (Cd) } & 0 \\ \text { Chromium (Cr) } & 10 \\ \text { Copper (Cu) } & 6 \\ \text { Iron (Fe) } & 30 \\ \text { Lead (Pb) } & 6\end{array}$

Manganese (Mn)

Mercury ( $\mathrm{Hg}$ )

Selenium ( $\mathrm{Se}$ )

Silver ( $\mathrm{Ag})$

Zinc $(\mathrm{Zn})$

30

6 Strontium, dissolved ( $\mathrm{Sr}) 1200$

10 $<.5$

0

0

ANALYSIS OF SELECTED PESTICIDE AND INDUSTRIAL COMPOUNDS

(Total concentrations in micrograms per liter)

$\begin{array}{llll}\text { Aldrin } & .00 & \text { Lindane } & .00 \\ \text { Chlordane } & .0 & \text { Methoxych1or } & .00 \\ \text { DDD } & .00 & \text { Mirex } & .00 \\ \text { DDE } & .00 & \text { PCB } & .0 \\ \text { DDT } & .00 & \text { PCN } & .00 \\ \text { Dieldrin } & .00 & \text { Silvex } & .00 \\ \text { Endrin } & .00 & \text { Toxaphene } & 0 \\ \text { Heptachlor } & .00 & 2,4-D & .00 \\ \text { Heptachlor epoxide } & .00 & 2,4,5-\mathrm{T} & .00\end{array}$

ANALYSIS OF SELECTED GROSS RADIOACTIVITY

(Total activity concentrations in picocuries per liter)

Gross Alpha, as

uranium natural 14

Gross Beta, as

cesium-137
Gross Beta, as

strontium -90

4.1 
PUBLIC WATER SUPPLY: Bunne 1

COUNTY: Flagler

COLLECTION DATE: 11-03-77

SAMPLING POINT (1) Treated water-292826081145801, tap in water treatment plant office on Dean Road.

(2) Raw water-

ANALYSIS OF MAJOR CHEMICAL CONSTITUENTS AND PHYSICAL PROPERTIES

(Dissolved concentrations in milligrams per liter, except as indicated)

Silica (SiO2)

Calcium ( $\mathrm{Ca}$ )

Magnesium ( $\mathrm{Mg})$

Sodium (Na)

Potassium ( $K$ )

Bicarbonate ( $\mathrm{HCO} 3)$

Carbonate ( $\mathrm{CO} 3$ )

Sulfate (S04)

Chloride (C1)

Fluoride (F)

Nitrate $($ NO3-N),

total

Nitrite (NO2-N),

total
(2)

$$
\begin{aligned}
& \text { Dissolved solids } \\
& \quad \text { (residue at } 180^{\circ} \mathrm{C} \text { ) } \\
& \text { Hardness as } \mathrm{CaCO} \\
& \quad \text { (Ca, Mg) } \\
& \text { Noncarbonate hardness } \\
& \text { as CaCO3 } \\
& \text { Percent sodium } \\
& \text { Alkalinity as CaCO } 3 \\
& \text { Specific conductance } \\
& \quad \text { (umhos/cm at } 25^{\circ} \mathrm{C} \text { ) } \\
& \text { pH (units) } \\
& \text { Temperature ( }{ }^{\circ} \mathrm{C} \text { ) } \\
& \text { Color (Pt-Co units) } \\
& \text { Turbidity (NTU) }
\end{aligned}
$$

ANALYSIS OF SELECTED TRACE ELEMENTS

(Total concentrations in micrograms per liter, except as indicated)

Arsenic (As)

Barium ( $\mathrm{Ba}$ )

Cadmium ( $C d$ )

Chromium (Cr)

Copper $(\mathrm{Cu})$

Iron ( $\mathrm{Fe})$

Lead $(\mathrm{Pb})$
Manganese $\left(\mathrm{Mn}_{\mathrm{n}}\right)$

Mercury ( $\mathrm{Hg})$

Selenium ( $\mathrm{Se}$ )

Silver ( $\mathrm{Ag})$

Strontium, dissolved (Sr)

Zinc $(\mathrm{Zn})$

ANALYSIS OF SELECTED PESTICIDE AND INDUSTRIAL COMPOUNDS

(Total concentrations in micrograms per liter)
Aldrin
Chlordane
DDD
DDE
DDT
Dieldrin
Endrin
Heptachlor
Heptachlor epoxide

Lindane
Methoxych lor
Mirex
PCB
PCN
Silvex
Toxaphene
2,4-D
2,4,5-T

Lindane

Methoxychlor

Mirex

PCB

PCN

Toxaphene

$2,4,5-T$

ANALYSIS OF SELECTED GROSS RADIOACTIVITY

(Total activity concentrations in picocuries per liter)

Gross Alpha, as

uranium natural $<4.3$

Gross Beta, as

cesium- 137
Gross Beta, as

strontium-90
(1) 
PUBLIC WATER SUPPLY: Callahan

COUNTY: Nassau

COLLECTION DATE: 12-14-77

SAMPLING POINT (1) Treated water-303347081494301, tap in restroom of City Hall on Kings Street.

(2) Raw water-

ANALYSIS OF MAJOR CHEMICAL CONSTITUENTS AND PHYSICAL PROPERTIES

(Dissolved concentrations in milligrams per liter, except as indicated)

(1)

36

66

30

18

2.0

160

0

120

42

.6

.01

.00
(2)

Dissolved solids

(residue at $180^{\circ} \mathrm{C}$ ) $\quad 400$

Hardness as $\mathrm{CaCO} 3$

( $\mathrm{Ca}, \mathrm{Mg}$ )

290

Noncarbonate hardness as $\mathrm{CaCO} 3$

160

Percent sodium

Alkalinity as $\mathrm{CaCO} 3$

Specific conductance

(umhos $/ \mathrm{cm}$ at $25^{\circ} \mathrm{C}$ )

$\mathrm{pH}$ (units)

Temperature $\left({ }^{\circ} \mathrm{C}\right)$

Color (Pt-Co units)

Turbidity (NTU)
(1)
Nitrate (NO3-N), total

total
$(\mathrm{NO2}-\mathrm{N})$,

(2)

\section{ANALYSIS OF SELECTED TRACE ELEMENTS}

(Total concentrations in micrograms per liter, except as indicated)

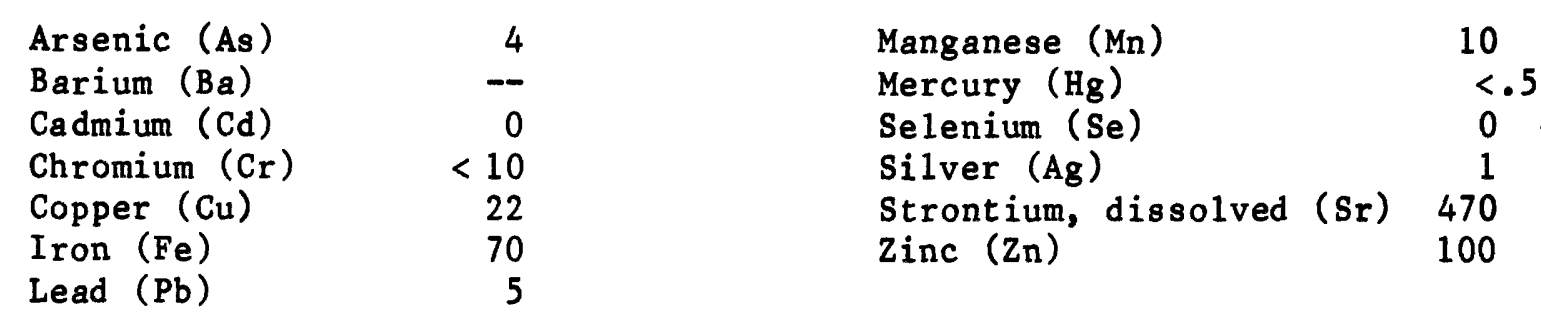

ANALYSIS OF SELECTED PESTICIDE AND INDUSTRIAL COMPOUNDS

(Total concentrations in micrograms per liter)

$\begin{array}{llll}\text { Aldrin } & .00 & \text { Lindane } & .00 \\ \text { Chlordane } & .0 & \text { Methoxych 1or } & .00 \\ \text { DDD } & .00 & \text { Mirex } & .00 \\ \text { DDE } & .00 & \text { PCB } & .0 \\ \text { DDT } & .00 & \text { PCN } & .00 \\ \text { Dieldrin } & .00 & \text { Silvex } & .00 \\ \text { Endrin } & .00 & \text { Toxaphene } & 0 \\ \text { Heptachlor } & .00 & 2,4-D & .00 \\ \text { Heptachlor epoxide } & .00 & 2,4,5-\mathrm{T} & .00\end{array}$

ANALYSIS OF SELECTED GROSS RADIOACTIVITY

(Total activity concentrations in picocuries per liter)

Gross Alpha, as

uranium natural $\quad 5.6$

Gross Beta, as

cesium- 137
Gross Beta, as

strontium-90 
PUBLIC WATER SUPPLY: Carrolwood

COUNTY: Hillsborough

COLLECTION DATE: 12-27-77

SAMPLING POINT (1) Treated water-280232082300809, tap outside pumphouse building (we11 number 4) on west side.

(2) Raw water-

ANALYSIS OF MAJOR CHEMICAL CONSTITUENTS AND PHYSICAL PROPERTIES (Dissolved concentrations in milligrams per liter, except as indicated)

$\begin{array}{lc}\text { Silica (SiO2) } & 13 \\ \text { Calcium (Ca) } & 74 \\ \text { Magnesium (Mg) } & 4.7 \\ \text { Sodium (Na) } & 5.2 \\ \text { Potassium (K) } & 5.1 \\ \text { Bicarbonate (HCO3) } & 230 \\ \text { Carbonate (CO3) } & 0 \\ \text { Sulfate (SO4) } & 1.7 \\ \text { Chloride (C1) } & 16 \\ \text { Fluoride (F) } & .1 \\ \text { Nitrate (NO3-N), } & \\ \quad \text { total (NO2-N), } & .00 \\ \text { Nitrite (NO2-N) } \\ \quad \text { total }\end{array}$

(2)

(1)

4.7

5.2

1.7

.1

.00

.00
Dissolved solids (residue at $180^{\circ} \mathrm{C}$ ) 228

Hardness as $\mathrm{CaCO} 3$

$(\mathrm{Ca}, \mathrm{Mg})$

Noncarbonate hardness as $\mathrm{CaCO} 3$

Percent sodium

Alkalinity as $\mathrm{CaCO} 3$

Specific conductance

(umhos $/ \mathrm{cm}$ at $25^{\circ} \mathrm{C}$ )

$\mathrm{pH}$ (units)

Temperature $\left({ }^{\circ} \mathrm{C}\right)$

Color (Pt-Co units)

Turbidity (NTU)
(1)

ANALYSIS OF SELECTED TRACE ELEMENTS

(Total concentrations in micrograms per liter, except as indicated)

$\begin{array}{lrlc}\text { Arsenic (As) } & 3 & \text { Manganese (Mn) } & 10 \\ \text { Barium (Ba) } & -- & \text { Mercury (Hg) } & <.5 \\ \text { Cadmium (Cd) } & 0 & \text { Selenium (Se) } & 0 \\ \text { Chromium (Cr) } & <10 & \text { Silver (Ag) } & 0 \\ \text { Copper (Cu) } & 4 & \text { Strontium, dissolved ( } \mathrm{Sr} \text { ) } & 110 \\ \text { Iron (Fe) } & 40 & \text { Zinc ( } \mathrm{nn} \text { ) } & 10 \\ \text { Lead (Pb) } & 0 & & \end{array}$

ANALYSIS OF SELECTED PESTICIDE AND INDUSTRIAL COMPOUNDS

(Total concentrations in micrograms per liter)

$\begin{array}{llll}\text { Aldrin } & .00 & \text { Lindane } & .00 \\ \text { Chlordane } & .0 & \text { Methoxychlor } & .00 \\ \text { DDD } & .00 & \text { Mirex } & .00 \\ \text { DDE } & .00 & \text { PCB } & .0 \\ \text { DDT } & .00 & \text { PCN } & .00 \\ \text { Dieldrin } & .00 & \text { Silvex } & .00 \\ \text { Endrin } & .00 & \text { Toxaphene } & 0 \\ \text { Heptachlor } & .00 & 2,4-D & .00 \\ \text { Heptachlor epoxide } & .00 & 2,4,5-\mathrm{T} & .00\end{array}$

ANALYSIS OF SELECTED GROSS RADIOACTIVITY

(Total activity concentrations in picocuries per liter)

Gross Alpha, as uranium natural $\quad 4.2$

Gross Beta, as

cesium-137
Gross Beta, as

strontium-90

1.9 
PUBLIC WATER SUPPLY: Century Village Utilities

COUNTY: Palm Beach

COLLECTION DATE: 11-30-77

SAMPLING POINT (1) Treated water -264228080073802 , tap in laboratory at water treatment plant.

(2) Raw water-

ANALYSIS OF MAJOR CHEMICAL CONSTITUENTS AND PHYSICAL PROPERTIES (Dissolved concentrations in milligrams per liter, except as indicated)

(1)

$\begin{array}{lc}\text { Silica ( } & 12 \\ \text { Calcium (Ca) } & 33 \\ \text { Magnesium (Mg) } & 4.5 \\ \text { Sodium (Na) } & 38 \\ \text { Potassium (K) } & 1.5 \\ \text { Bicarbonate (HCO3) } & 56 \\ \text { Carbonate (CO3) } & 0 \\ \text { Sulfate (SO4) } & 13 \\ \text { Chloride (C1) } & 83 \\ \text { Fluoride (F) } & .2 \\ \text { Nitrate (NO3-N), } & \\ \quad \text { total (NO2-N), } & .02 \\ \text { Nitrite (NO2-N), } & \\ \quad \text { total } & .01\end{array}$

(2)

(1)

Dissolved solids

(residue at $180^{\circ} \mathrm{C}$ ) 260

Hardness as $\mathrm{CaCO} 3$

$(\mathrm{Ca}, \mathrm{Mg})$

100

Noncarbonate hardness as $\mathrm{CaCO}_{3}$

54

Percent sodium 45

Alkalinity as $\mathrm{CaCO} 3 \quad 46$

Specific conductance

(umhos $/ \mathrm{cm}$ at $25^{\circ} \mathrm{C}$ )

385

$\mathrm{pH}$ (units)

8.1

Temperature $\left({ }^{\circ} \mathrm{C}\right)$

Color (Pt-Co units)

Turbidity (NTU)

ANALYSIS OF SELECTED TRACE ELEMENTS

(Total concentrations in micrograms per liter, except as indicated)

$\begin{array}{lr}\text { Arsenic (As) } & 0 \\ \text { Barium (Ba) } & 0 \\ \text { Cadmium (Cd) } & 1 \\ \text { Chromium (Cr) } & 10 \\ \text { Copper (Cu) } & 3 \\ \text { Iron (Fe) } & 10 \\ \text { Lead (Pb) } & 27\end{array}$

Manganese (Mn)

Mercury ( $\mathrm{Hg})$

Selenium ( $\mathrm{Se}$ )

Silver $(\mathrm{Ag})$

Strontium, dissolved ( $\mathrm{Sr}$ ) 760

Zinc $(\mathrm{Zn})$

ANALYSIS OF SELECTED PESTICIDE AND INDUSTRIAL COMPOUNDS

(Total concentrations in micrograms per liter)

$\begin{array}{llll}\text { Aldrin } & .00 & \text { Lindane } & .00 \\ \text { Chlordane } & .0 & \text { Methoxychlor } & .00 \\ \text { DDD } & .00 & \text { Mirex } & .00 \\ \text { DDE } & .00 & \text { PCB } & .0 \\ \text { DDT } & .00 & \text { PCN } & .00 \\ \text { Dieldrin } & .00 & \text { Silvex } & .00 \\ \text { Endrin } & .00 & \text { Toxaphene } & 0 \\ \text { Heptachlor } & .00 & 2,4-\mathrm{D} & .00 \\ \text { Heptachlor epoxide } & .00 & 2,4,5-\mathrm{T} & .00\end{array}$

ANALYSIS OF SELECTED GROSS RADIOACTIVITY

(Total activity concentrations in picocuries per liter)

Gross Alpha, as uranium natural

Gross Beta, as

cesium-137
Gross Beta, as

strontium-90 
PUBLIC WATER SUPPLY: Chattahoochee COUNTY: Gadsden COLLECTION DATE: 12-02-77

SAMPLING POINT (1) Treated water-304217084493790, tap on west side of unit 3 building at the Florida State Hospital (Mosquito Creek).

(2) Raw water-

ANALYSIS OF MAJOR CHEMICAL CONSTITUENTS AND PHYSICAL PROPERTIES (Dissolved concentrations in milligrams per liter, except as indicated)

(1) (2)

Silica (SiO2)

Calcium $(\mathrm{Ca})$

Magnesium ( $\mathrm{Mg}$ )

Sodium $(\mathrm{Na})$

Potassium ( $\mathrm{K}$ )

Bicarbonate ( $\mathrm{HCO})$

Carbonate ( $\mathrm{CO} 3$ )

Sulfate ( 504 )

Chloride (C1)

Fluoride (F)

Nitrate (NO3-N), total

Nitrite (NO2-N),

total
6.1

17

1.4

2.5

.6

30

1

12

11

.0

.14

.00
Dissolved solids

(residue at $180^{\circ} \mathrm{C}$ ) $\quad 79$

Hardness as $\mathrm{CaCO} 3$

$(\mathrm{Ca}, \mathrm{Mg}) \quad 48$

Noncarbonate hardness as $\mathrm{CaCO} 3$

22

Percent sodium 10

Alkalinity as $\mathrm{CaCO} 3 \quad 26$

Specific conductance

(umhos $/ \mathrm{cm}$ at $25^{\circ} \mathrm{C}$ )

$\mathrm{pH}$ (units)

95

Temperature $\left({ }^{\circ} \mathrm{C}\right)$

Color (Pt-Co units)

Turbidity (NTU)
(1)

ANALYSIS OF SELECTED TRACE ELEMENTS

(Total concentrations in micrograms per liter, except as indicated)

$\begin{array}{lclc}\text { Arsenic (As) } & 0 & \text { Manganese (Mn) } & 10 \\ \text { Barium (Ba) } & 0 & \text { Mercury (Hg) } & <.5 \\ \text { Cadmium (Cd) } & 1 & \text { Selenium (Se) } & 0 \\ \text { Chromium (Cr) } & 10 & \text { Silver (Ag) } & 0 \\ \text { Copper (Cu) } & 50 & \text { Strontium, dissolved ( } \mathrm{Sr} \text { ) } & 60 \\ \text { Iron (Fe) } & 50 & \text { Zinc ( } \mathrm{n} \text { ) } & 10 \\ \text { Lead (Pb) } & 14 & & \end{array}$

ANALYSIS OF SELECTED PESTICIDE AND INDUSTRIAL COMPOUNDS

(Total concentrations in micrograms per liter)

$\begin{array}{llll}\text { Aldrin } & .00 & \text { Lindane } & .00 \\ \text { Chlordane } & .0 & \text { Methoxychlor } & .00 \\ \text { DDD } & .00 & \text { Mirex } & .00 \\ \text { DDE } & .00 & \text { PCB } & .0 \\ \text { DDT } & .00 & \text { PCN } & .00 \\ \text { Dieldrin } & .00 & \text { Silvex } & .00 \\ \text { Endrin } & .00 & \text { Toxaphene } & 0 \\ \text { Heptachlor } & .00 & 2,4-D & .00 \\ \text { Heptachlor epoxide } & .00 & 2,4,5-T & .00\end{array}$

ANALYSIS OF. SELECTED GROSS RADIOACTIVITY

(Total activity concentrations in picocuries per liter)

Gross Alpha, as

uranium natural $<1.0$

Gross Beta, as

cesium-137
Gross Beta, as

strontium -90

0.9 
PUBLIC WATER SUPPLY: Chiefland COUNTY: LevY COLLECTION DATE: 11-08-77

SAMPLING POINT (1) Treated water-292844082513301, tap outside pump house.

(2) Raw water-

ANALYSIS OF MAJOR CHEMICAL CONSTITUENTS AND PHYSICAL PROPERTIES (Dissolved concentrations in milligrams per 1iter, except as indicated)
(1)
(2)
(1)

Silica ( $\mathrm{SiO2)}$

Calcium ( $\mathrm{Ca}$ )

Magnesium ( $\mathrm{Mg})$

Sodium ( Na)

Potassium (K)

Bicarbonate ( $\mathrm{HCO} 3)$--

Carbonate ( $\mathrm{CO} 3)$

Sulfate (s04)

Chloride (C1)

Fluoride (F)

Nitrate (NO3-N), total

Nitrite (NO2-N),

total
5.3

76

3.1

4.3

.8

0

24

8.6

.1

2.3

.00
Dissolved solids

(residue at $180^{\circ} \mathrm{C}$ ) 226

Hardness as $\mathrm{CaCO} 3$

$(\mathrm{Ca}, \mathrm{Mg}) \quad 200$

Noncarbonate hardness as $\mathrm{CaCO} 3$

Percent sodium

Alkalinity as $\mathrm{CaCO} 3$

Specific conductance

(umhos $/ \mathrm{cm}$ at $25^{\circ} \mathrm{C}$ )

$\mathrm{pH}$ (units)

Temperature $\left({ }^{\circ} \mathrm{C}\right)$

Color (Pt-Co units)

Turbidity (NTU)
39

4

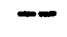

406

7.4

24.0

1

0

ANALYSIS OF SELECTED TRACE ELEMENTS

(Total concentrations in micrograms per liter, except as indicated)

Arsenic (As)

Barium ( $B a$ )

Cadmium (Cd)

Chromium (Cr)

Copper ( $\mathrm{Cu}$ )

Iron ( $\mathrm{Fe}$ )

Lead $(\mathrm{Pb})$

1
0
0
$<10$
7
40
0

Manganese (Mn)

Mercury ( $\mathrm{Hg}$ )

Selenium (Se)

Silver (Ag)

Strontium, dissolved (Sr) 160

Zinc $(\mathrm{Zn})$
3

$<.5$

0

0

10

ANALYSIS OF SELECTED PESTICIDE AND INDUSTRIAL COMPOUNDS

(Total concentrations in micrograms per liter)

Aldrin

Chlordane

DDD

DDE

DDT

Dieldrin

Endrin

Heptachlor

Heptachlor epoxide

.00
.0
.00
.00
.00
.00
.00
.00
.00

Lindane

Methoxych lor

Mirex

PCB

PCN

Silvex

Toxaphene

2,4-D

$2,4,5-T$

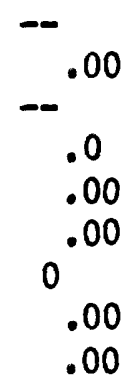

ANALYSIS OF SELECTED GROSS RADIOACTIVITY

(Total activity concentrations in picocuries per liter)

Gross Alpha, as

uranium natural $<2.6$
Gross Beta, as

strontium-90 
PUBLIC WATER SUPPLY: Clermont

COUNTY: Lake

COLLECTION DATE: 11-03-77

SAMPLING POINT (1) Treated water-283301081445901, tap inside 1aboratory water treatment plant.

(2) Raw water-

ANALYSIS OF MAJOR CHEMICAL CONSTITUENTS AND PHYSICAL PROPERTIES

(Dissolved concentrations in milligrams per liter, except as indicated)

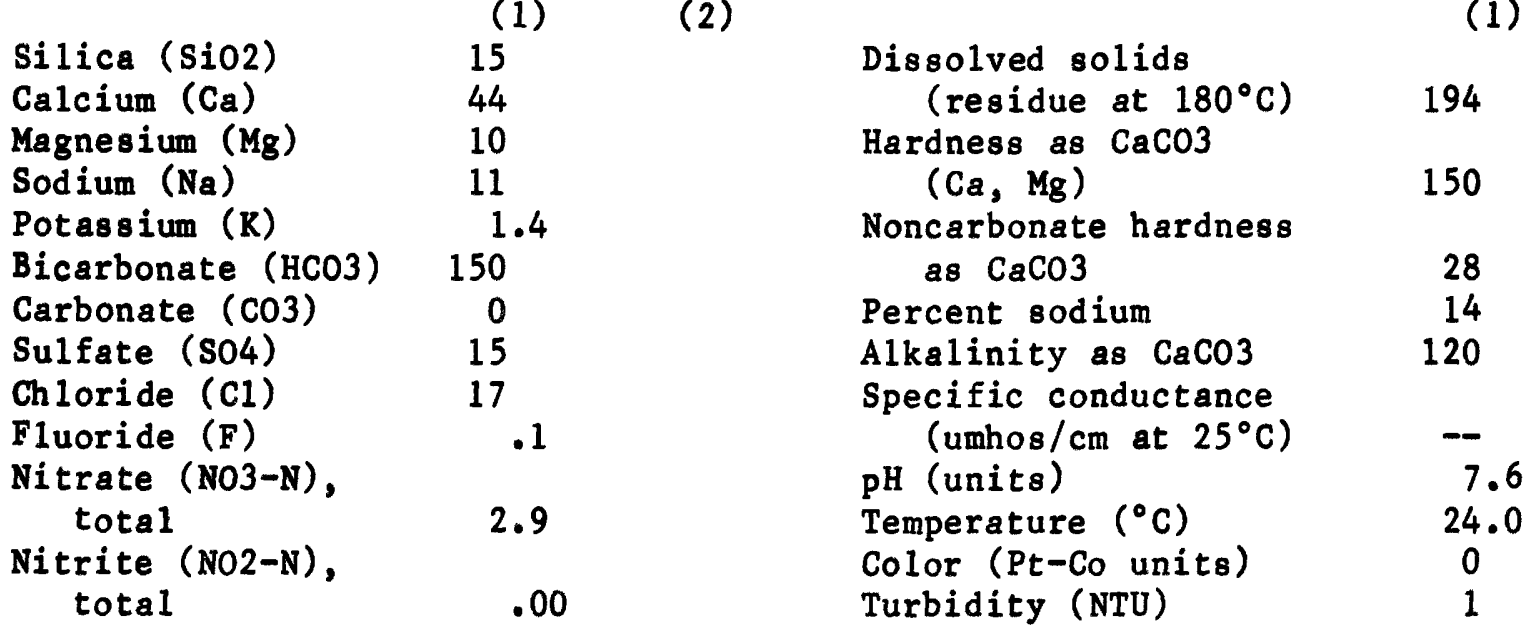

ANALYSIS OF SELECTED TRACE ELEMENTS

(Total concentrations in micrograms per liter, except as indicated)

$\begin{array}{lrlr}\text { Arsenic (As) } & 0 & \text { Manganese (Mn) } & 0 \\ \text { Barium (Ba) } & 100 & \text { Mercury (Hg) } & <.5 \\ \text { Cadmium (Cd) } & 0 & \text { Selenium (Se) } & 0 \\ \text { Chromium (Cr) } & 10 & \text { Silver (Ag) } & 0 \\ \text { Copper (Cu) } & 1 & \text { Strontium, dissolved ( } \mathrm{Sr}) & 100 \\ \text { Iron ( } \mathrm{Fe}) & 40 & \text { Zinc }(\mathrm{Zn}) & 40 \\ \text { Lead (Pb) } & 7 & & \end{array}$

ANALYSIS OF SELECTED PESTICIDE AND INDUSTRIAL COMPOUNDS

(Total concentrations in micrograms per liter)

$\begin{array}{llll}\text { Aldrin } & - & \text { Lindane } & - \\ \text { Chlordane } & - & \text { Methoxychlor } & - \\ \text { DDD } & - & \text { Mirex } & - \\ \text { DDE } & -- & \text { PCB } & - \\ \text { DDT } & - & \text { PCN } & . \\ \text { Dieldrin } & - & \text { Silvex } & .00 \\ \text { Endrin } & -- & \text { Toxaphene } & .00 \\ \text { Heptachlor } & -- & 2,4-D & .00 \\ \text { Heptachlor epoxide } & - & 2,4,5-\mathrm{T} & \end{array}$

ANALYSIS OF SELECTED GROSS RADIOACTIVITY

(Total activity concentrations in picocuries per liter)

Gross Alpha, as

uranium natural -

Gross Beta, as

cesium-137
Gross Beta, as

strontium -90 
PUBLIC WATER SUPPLY: Clewiston

COUNTY: Hendry

COLLECTION DATE: 02-02-78

SAMPLING POINT (1) Treated water-264417080560802, tap at Benbow Village, Moorehaven

(Glades County).

(2) Raw water-264417080560701, (U.S. Sugar Corporation) Clewiston water treatment plant intake from canal at Lake Okeechobee. ANALYSIS OF MAJOR CHEMICAL CONSTITUENTS AND PHYSICAL PROPERTIES

(Dissolved concentrations in milligrams per liter, except as indicated)

(1) (2)

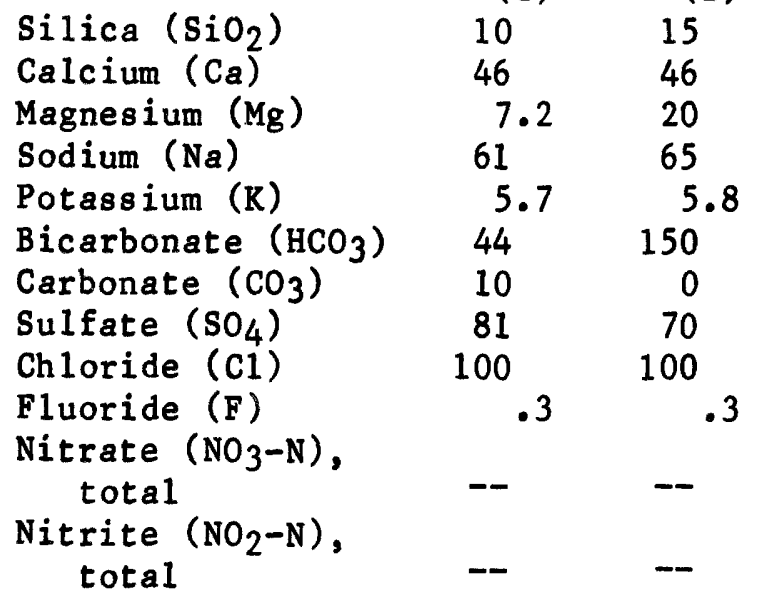

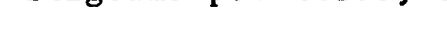

Dissolved solids (residue at $180^{\circ} \mathrm{C}$ )

Hardness as $\mathrm{CaCO}_{3}$

$(\mathrm{C} a, \mathrm{Mg})$

Noncarbonate hardness

(1)

$\begin{array}{lcc}\quad \text { as } \mathrm{CaCO}_{3} & 93 & 76 \\ \text { Percent sodium } & 47 & 41 \\ \text { Alkalinity as } \mathrm{CaCO}_{3} & 53 & 120 \\ \text { Specific conductance } & & \\ \quad \text { (umhos/cm at } 25^{\circ} \mathrm{C} \text { ) } & 616 & -- \\ \text { pH (units) } & 10.0 & 8.2 \\ \text { Temperature ( }{ }^{\circ} \mathrm{C} \text { ) } & 20.5 & 17.5 \\ \text { Color (Pt-Co units) } & 10 & 150 \\ \text { Turbidity (NTU) } & -- & --\end{array}$

ANALYSIS OF SELECTED TRACE ELEMENTS

(Total concentrations in micrograms per liter, except as indicated)

$\begin{array}{lrrlrr}\text { Arsenic (As) } & 0 & 1 & \text { Manganese (Mn) } & 10 & 30 \\ \text { Barium (Ba) } & 0 & 100 & \text { Mercury (Hg) } & <.5 & <.5 \\ \text { Cadmium (Cd) } & 1 & 1 & \text { Selenium (Se) } & 0 & 0 \\ \text { Chromium (Cr) } & 20 & 20 & \text { Silver (Ag) } & 0 & 0 \\ \text { Copper (Cu) } & 5 & 21 & \text { Strontium, dissolved (Sr) } & 740 & 1200 \\ \text { Iron (Fe) } & 40 & 1000 & \text { Zinc (Zn) } & 10 & 10 \\ \text { Lead (Pb) } & 5 & 19 & & & \end{array}$

ANALYSIS OF SELECTED PESTICIDE AND INDUSTRIAL COMPOUNDS

(Total concentrations in micrograms per liter)

$\begin{array}{llllll}\text { Aldrin } & .00 & .00 & \text { Lindane } & .00 & .00 \\ \text { Chlordane } & .0 & .0 & \text { Methoxychlor } & .00 & .00 \\ \text { DDD } & .00 & .00 & \text { Mirex } & .00 & .00 \\ \text { DDE } & .00 & .00 & \text { PCB } & .0 & .0 \\ \text { DDT } & .00 & .00 & \text { PCN } & .00 & .00 \\ \text { Dieldrin } & .00 & .00 & \text { Silvex } & .00 & .00 \\ \text { Endrin } & .00 & .00 & \text { Toxaphene } & 0 & 0 \\ \text { Heptachlor } & .00 & .00 & 2,4-D & .00 & .00 \\ \text { Heptachlor epoxide } & .00 & .00 & 2,4,5-\mathrm{T} & .00 & .00\end{array}$

ANALYSIS OF SELECTED GROSS RADIOACTIVITY

(Total activity concentrations in picocuries per liter)

Gross Alpha, as uranium natural

Gross Beta, as

cesium-137
$<4.3$

13
Gross Beta, as

strontium-90

$-$

12 
PUBLIC WATER SUPPLY: Collier Estates

COUNTY: Broward

COLLECTION DATE: 11-23-77

SAMPLING POINT (1) Treated water-261120080080401, tap at store, Northridge

Shopping Center.

(2) Raw water-

ANALYSIS OF MAJOR CHEMICAL CONSTITUENTS AND PHYSICAL PROPERTIES

(Dissolved concentrations in milligrams per liter, except as indicated)

(1) (2)

$\begin{array}{lc}\text { Silica (SiO2) } & 9.2 \\ \text { Calcium (Ca) } & 30 \\ \text { Magnesium (Mg) } & 7.4 \\ \text { Sodium (Na) } & 41 \\ \text { Potassium (K) } & 2.8 \\ \text { Bicarbonate (HCO3) } & 83 \\ \text { Carbonate (CO3) } & 0 \\ \text { Sulfate (SO4) } & 14 \\ \text { Chloride (C1) } & 84 \\ \text { Fluoride (F) } & .5 \\ \text { Nitrate (NO3-N), } & \\ \quad \text { total (NO2-N), } & .06 \\ \text { Nitrite } \quad \\ \quad \text { total }\end{array}$

(1)
Dissolved solids
(residue at 180 )

Hardness as $\mathrm{CaCO} 3$

$(\mathrm{Ca}, \mathrm{Mg}) \quad 110$

Noncarbonate hardness as $\mathrm{CaCO} 3$

Percent sodium

Alkalinity as $\mathrm{CaCO} 3$

Specific conductance

(umhos $/ \mathrm{cm}$ at $25^{\circ} \mathrm{C}$ )

$\mathrm{pH}$ (units)

Temperature $\left({ }^{\circ} \mathrm{C}\right)$

Color (Pt-Co units)

Turbidity (NTU)
38

251

45

68

440

8.2

26.5

10

1

ANALYSIS OF SELECTED TRACE ELEMENTS

(Total concentrations in micrograms per liter, except as indicated)

$\begin{array}{lrlr}\text { Arsenic (As) } & 1 & \text { Manganese (Mn) } & 10 \\ \text { Barium (Ba) } & - & \text { Mercury (Hg) } & .6 \\ \text { Cadmium (Cd) } & 1 & \text { Selenium (Se) } & 0 \\ \text { Chromium (Cr) } & 10 & \text { Silver (Ag) } & 0 \\ \text { Copper ( Cu) } & 9 & \text { Strontium, dissolved (Sr) } & 330 \\ \text { Iron (Fe) } & 190 & \text { Zinc (Zn) } & 110 \\ \text { Lead ( } \mathrm{Pb}) & 18 & & \end{array}$

ANALYSIS OF SELECTED PESTICIDE AND INDUSTRIAL COMPOUNDS

( Total concentrations in micrograms per 1iter)

$\begin{array}{llll}\text { Aldrin } & .00 & \text { Lindane } & .00 \\ \text { Chlordane } & .0 & \text { Methoxychlor } & .00 \\ \text { DDD } & .00 & \text { Mirex } & .00 \\ \text { DDE } & .00 & \text { PCB } & .0 \\ \text { DDT } & .00 & \text { PCN } & .00 \\ \text { Dieldrin } & .00 & \text { Silvex } & .14 \\ \text { Endrin } & .00 & \text { Toxaphene } & 0 \\ \text { Heptachlor } & .00 & 2,4-D & .00 \\ \text { Heptachlor epoxide } & .00 & 2,4,5-T & .00\end{array}$

ANALYSIS OF SELECTED GROSS RADIOACTIVITY

(Total activity concentrations in picocuries per liter)

Gross Alpha, as

uranium natural --

Gross Beta, as

cesium-137
Gross Beta, as

strontium -90 
PUBLIC WATER SUPPLY: Colonial Hills

COUNTY: Pasco

COLLECTION DATE: 12-20-77

SAMPLING POINT (1) Treated water-281252082434609, tap on holding tank near pumphouse (well number 2).

(2) Raw water-

ANALYSIS OF MAJOR CHEMICAL CONSTITUENTS AND PHYSICAL PROPERTIES

(Dissolved concentrations in milligrams per liter, except as indicated)

$\begin{array}{lc}\text { Silica (SiO2) } & 16 \\ \text { Calcium (Ca) } & 95 \\ \text { Magnesium (Mg) } & 15 \\ \text { Sodium (Na) } & 50 \\ \text { Potassium (K) } & 3.3 \\ \text { Bicarbonate (HCO3) } & 160 \\ \text { Carbonate (CO3) } & 0 \\ \text { Sulfate (S04) } & 74 \\ \text { Chloride (C1) } & 130 \\ \text { Fluoride (F) } & .1 \\ \text { Nitrate (NO3-N), } \\ \quad \text { tota1 (NO2-N), } \\ \text { Nitrite } \quad \\ \quad \text { total }\end{array}$

(2)

(1)

(1)

95

15

3.3

160

74

130

.1

\section{7}

.00
Dissolved solids

$$
\text { (residue at } 180^{\circ} \mathrm{C} \text { ) } 524
$$

Hardness as $\mathrm{CaCO} 3$

$$
(\mathrm{Ca}, \mathrm{Mg})
$$

Noncarbonate hardness as $\mathrm{CaCO} 3$

170

Percent sodium 26

Alkalinity as $\mathrm{CaCO} 3 \quad 130$

Specific conductance

(umhos $/ \mathrm{cm}$ at $25^{\circ} \mathrm{C}$ )

$\mathrm{pH}$ (units)

Temperature $\left({ }^{\circ} \mathrm{C}\right)$

Color (Pt-Co units)

Turbidity (NTU)

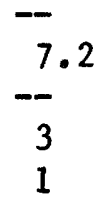

ANALYSIS OF SELECTED TRACE ELEMENTS

(Total concentrations in micrograms per liter, except as indicated)

$\begin{array}{lrlr}\text { Arsenic (As) } & 2 & \text { Manganese (Mn) } & 10 \\ \text { Barium (Ba) } & -- & \text { Mercury (Hg) } & <.5 \\ \text { Cadmium (Cd) } & 0 & \text { Selenium (Se) } & 6 \\ \text { Chromium (Cr) } & 10 & \text { Silver (Ag) } & 2 \\ \text { Copper ( } & 4 \text { Strontium, dissolved (Sr) } & 180 \\ \text { Iron (Fe) } & 4 & \text { Zinc (Zn) } & 10 \\ \text { Lead ( } \mathrm{Pb}) & 20 & & \end{array}$

ANALYSIS OF SELECTED PESTICIDE AND INDUSTRIAL COMPOUNDS

(Total concentrations in micrograms per liter)

$\begin{array}{llll}\text { Aldrin } & .00 & \text { Lindane } & .00 \\ \text { Chlordane } & .0 & \text { Methoxychlor } & .00 \\ \text { DDD } & .00 & \text { Mirex } & .00 \\ \text { DDE } & .00 & \text { PCB } & .0 \\ \text { DDT } & .00 & \text { PCN } & .00 \\ \text { Dieldrin } & .00 & \text { Silvex } & .00 \\ \text { Endrin } & .00 & \text { Toxaphene } & 0 \\ \text { Heptachlor } & .00 & 2,4-D & .00 \\ \text { Heptachlor epoxide } & .00 & 2,4,5-T & .00\end{array}$

ANALYSIS OF SELECTED GROSS RADIOACTIVITY

(Total activity concentrations in picocuries per liter)

Gross Alpha, as uranium natural 41

Gross Beta, as

cesium-137
Gross Beta, as

strontium -90

13 
PUBLIC WATER SUPPLY: Conway

COUNTY: Orange

COLLECTION DATE: $11-14-77$

SAMPLING POINT (1) Treated water-282912081181501, tap at First Florida Utilities water treatment plant at Conway.

(2) Raw water-

ANALYSIS OF MAJOR CHEMICAL CONSTITUENTS AND PHYSICAL PROPERTIES (Dissolved concentrations in milligrams per liter, except as indicated)

(1) (2)

\begin{tabular}{|c|c|}
\hline $\begin{array}{l}\text { Silica ( } \mathrm{SiO} 2) \\
\text { Calcium ( } \mathrm{Ca}) \\
\text { Magnesium (Mg) } \\
\text { Sodium (Na) } \\
\text { Potassium (K) } \\
\text { Bicarbonate (HCO3) } \\
\text { Carbonate ( } \mathrm{CO} 3) \\
\text { Sulfate ( } \mathrm{SO} 4) \\
\text { Chloride ( } 1) \\
\text { Fluoride (F) } \\
\text { Nitrate (NO3-N), } \\
\text { total } \\
\text { Nitrite (NO2-N), } \\
\text { total }\end{array}$ & $\begin{array}{c}11 \\
41 \\
7.0 \\
9.6 \\
1.3 \\
150 \\
0 \\
7.8 \\
20 \\
.2\end{array}$ \\
\hline
\end{tabular}

Dissolved solids (residue at $180^{\circ} \mathrm{C}$ )

Hardness as $\mathrm{CaCO} 3$ $(\mathrm{Ca}, \mathrm{Mg})$

(1)

168

130

Noncarbonate hardness as $\mathrm{CaCO} 3$

Percent sodium

Alkalinity as $\mathrm{CaCO} 3$

Specific conductance

(umhos $/ \mathrm{cm}$ at $25^{\circ} \mathrm{C}$ )

$\mathrm{pH}$ (units)

Temperature $\left({ }^{\circ} \mathrm{C}\right)$

Color (Pt-Co units)

Turbidity (NTU)

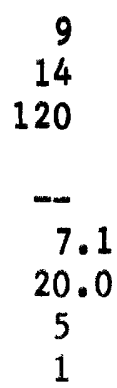

ANALYSIS OF SELECTED TRACE ELEMENTS

(Total concentrations in micrograms per liter, except as indicated)
Manganese (Mn)

Mercury ( $\mathrm{Hg}$ )

Selenium ( $\mathrm{Se}$ )

Silver (Ag)

Strontium, dissolved ( $\mathrm{Sr}$ ) 490

Zinc $(\mathrm{Zn})$
10 $<.5$ 0 0 130

ANALYSIS OF SELECTED PESTICIDE AND INDUSTRIAL COMPOUNDS (Total concentrations in micrograms per 1iter)

$\begin{array}{llll}\text { Aldrin } & .00 & \text { Lindane } & .00 \\ \text { Chlordane } & .0 & \text { Methoxychlor } & .00 \\ \text { DDD } & .00 & \text { Mirex } & .00 \\ \text { DDE } & .00 & \text { PCB } & .0 \\ \text { DDT } & .00 & \text { PCN } & .00 \\ \text { Dieldrin } & .00 & \text { Silvex } & .00 \\ \text { Endrin } & .00 & \text { Toxaphene } & 0 \\ \text { Heptachlor } & .00 & 2,4-D & .00 \\ \text { Heptachlor epoxide } & .00 & 2,4,5-T & .00\end{array}$

ANALYSIS OF SELECTED GROSS RADIOACTIVITY

(Total activity concentrations in picocuries per liter)

Gross Alpha, as

uranium natural $<2.3$

Gross Beta, as

cesium-137
Gross Beta, as

strontium -90
.00

.00

.0

.00

.00

.00 
PUBLIC WATER SUPPLY: Crescent City COUNTY: Putnam COLLECTION DATE: 11-08-77

SAMPLING POINT (1) Treated water-292546081305901, tap outside water treatment plant office.

(2) Raw water-

ANALYSIS OF MAJOR CHEMICAL CONSTITUENTS AND PHYSICAL PROPERTIES (Dissolved concentrations in milligrams per liter, except as indicated)

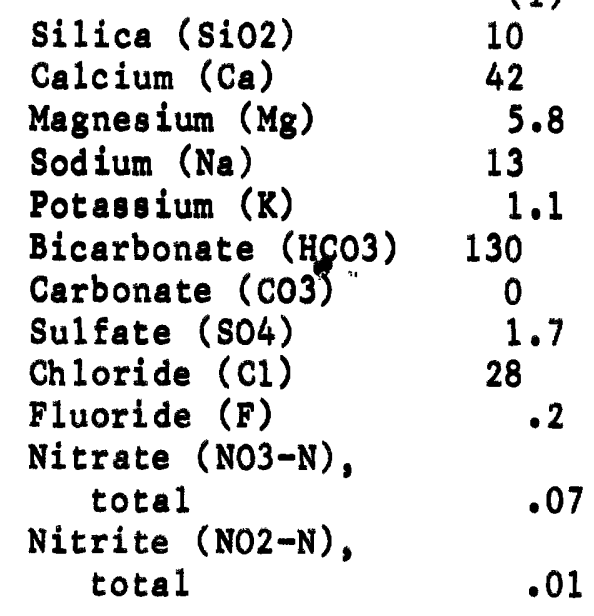
(2)

(1)

Dissolved solids (residue at $180^{\circ} \mathrm{C}$ ) $\quad 187$

Hardness as $\mathrm{CaCO}$ $(\mathrm{Ca}, \mathrm{Mg})$

130

Noncarbonate hardness as $\mathrm{CaCO}_{3}$

22

Percent sodium 18

Alkalinity as $\mathrm{CaCO} 3 \quad 110$

Specific conductance (umhos $/ \mathrm{cm}$ at $25^{\circ} \mathrm{C}$ )

$\mathrm{pH}$ (units)

7.3

Temperature $\left({ }^{\circ} \mathrm{C}\right) \quad 23.5$

Color (Pt-Co units)

Turbidity (NTU)

2

1

ANALYSIS OF SELECTED TRACE ELEMENTS

(Total concentrations in micrograms per liter, except as indicated)

$\begin{array}{lrlc}\text { Arsenic (As) } & 0 & \text { Manganese (Mn) } & 10 \\ \text { Barium (Ba) } & 0 & \text { Mercury ( } \mathrm{Hg}) & <.5 \\ \text { Cadmium (Cd) } & 1 & \text { Selenium ( } \mathrm{Se}) & 0 \\ \text { Chromium (Cr) } & <10 & \text { Silver (Ag) } & 1 \\ \text { Copper (Cu) } & 80 & \text { Strontium, dissolved ( } \mathrm{Sr} \text { ) } & 200 \\ \text { Iron (Fe) } & 260 & \text { Zinc ( } \mathrm{n} \text { ) } & 20 \\ \text { Lead (Pb) } & 5 & & \end{array}$

ANALYSIS OF SELECTED PESTICIDE AND INDUSTRIAL COMPOUNDS (Total concentrations in micrograms per liter)

$\begin{array}{llll}\text { Aldrin } & .00 & \text { Lindane } & .00 \\ \text { Chlordane } & .0 & \text { Methoxychlor } & .00 \\ \text { DDD } & .00 & \text { Mirex } & .00 \\ \text { DDE } & .00 & \text { PCB } & .0 \\ \text { DDT } & .00 & \text { PCN } & .00 \\ \text { Dieldrin } & .00 & \text { Silvex } & .00 \\ \text { Endrin } & .00 & \text { Toxaphene } & 0 \\ \text { Heptachlor } & .00 & 2,4-D & .00 \\ \text { Heptachlor epoxide } & .00 & 2,4,5-T & .00\end{array}$

ANALYSIS OF SELECTED GROSS RADIOACTIVITY

(Total activity concentrations in picocuries per liter)

Gross Alpha, as

uranium natural $<10$

Gross Beta, as

cesium-137
Gross Beta, as

strontium -90
.00

.00

.00

.0

.00

.00

.00 
PUBLIC WATER SUPPLY: Crystal River

COUNTY: Citrus

COLLECTION DATE: $12-19-77$

SAMPLING POINT (1) Treated water-285356082352809, tap on east side of well building (at 123 northwest Highway 19).

(2) Raw water-

ANALYSIS OF MAJOR CHEMICAL CONSTITUENTS AND PHYSICAL PROPERTIES (Dissolved concentrations in milligrams per liter, except as indicated)

(1) (2)

11

27

5.4

2.7

.3

85

0

14

4.9

.1

.15

.00

\section{Dissolved solids}

(residue at $180^{\circ} \mathrm{C}$ )

Hardness as $\mathrm{CaCO} 3$

$(\mathrm{Ca}, \mathrm{Mg})$

(1)

113

$\begin{array}{lc}\text { Carbonate (CO3) } & 0 \\ \text { Sulfate (SO4) } & 14 \\ \text { Chloride (C1) } & 4.9 \\ \text { Fluoride (F) } & .1 \\ \text { Nitrate (NO3-N), } & \\ \quad \text { total } & .15 \\ \text { Nitrite (NO2-N), } & \\ \quad \text { total } & .00\end{array}$

Noncarbonate hardness

as $\mathrm{CaCO} 3$

Percent sodium

Alkalinity as $\mathrm{CaCO} 3$

Specific conductance

(umhos $/ \mathrm{cm}$ at $25^{\circ} \mathrm{C}$ )

(Total concentrations in micrograms per liter, except as indicated)

Arsenic (As)

Barium ( $\mathrm{Ba}$ )

Cadmium (Cd)

Chromium ( $\mathrm{Cr}$ )

Copper $(\mathrm{Cu})$

Iron ( $\mathrm{Fe}$ )

Lead $(\mathrm{Pb})$
$\mathrm{pH}$ (units)

Temperature $\left({ }^{\circ} \mathrm{C}\right)$

Color (Pt-Co units)

Turbidity (NTU)

ANALYSIS OF SELECTED TRACE ELEMENTS

0
-
0
10
4
20
10

Manganese (Mn)

Mercury ( $\mathrm{Hg}$ )

Selenium ( $\mathrm{Se}$ )

Silver ( $\mathrm{Ag})$

Strontium, dissolved ( $\mathrm{Sr}$ ) 110

Zinc $(\mathrm{Zn})$

0

$<.5$

0

0

7.5

3

1

ANALYSIS OF SELECTED PESTICIDE AND INDUSTRIAL COMPOUNDS

(Total concentrations in micrograms per liter)

$\begin{array}{llll}\text { Aldrin } & .00 & \text { Lindane } & .00 \\ \text { Chlordane } & .0 & \text { Methoxychlor } & .00 \\ \text { DDD } & .00 & \text { Mirex } & .00 \\ \text { DDE } & .00 & \text { PCB } & .0 \\ \text { DDT } & .00 & \text { PCN } & .00 \\ \text { Dieldrin } & .00 & \text { Silvex } & .00 \\ \text { Endrin } & .00 & \text { Toxaphene } & 0 \\ \text { Heptachlor } & .00 & 2,4-D & .00 \\ \text { Heptachlor epoxide } & .00 & 2,4,5-\mathrm{T} & .00\end{array}$

ANALYSIS OF SELECTED GROSS RADIOACTIVITY

(Total activity concentrations in picocuries per liter)

Gross Alpha, as

uranium natural $<1.0$

Gross Beta, as

cesium-137
Gross Beta, as

strontium -90
$<.4$ 
PUBLIC WATER SUPPLY: Davie

COUNTY: Broward

COLLECTION DATE: 11-22-77

SAMPLING POINT (1) Treated water-260243080133001, tap inside Davie Water Treatment plant, 68 th Avenue and Sterling Road.

(2) Raw water-

ANALYSIS OF MAJOR CHEMICAL CONSTITUENTS AND PHYSICAL PROPERTIES

(Dissolved concentrations in miliigrams per liter, except as indicated)

(1) (2)

Silica (si02)

Calcium ( $\mathrm{Ca}$ )

Magnesium ( $\mathrm{Mg}$ )

Sodium $(\mathrm{Na})$

Potassium (K)

Bicarbonate ( $\mathrm{HCO} 3$ )

Carbonate ( $\mathrm{CO} 3$ )

Sulfate ( 504 )

Chloride (C1)

Fluoride (F)

Nitrate (NO3-N),

total

Nitrite ( NO2-N),

total
9.6

37

3.2

12

2.0

97

0

11

32

.2

.01

.00
Dissolved solids (residue at $180^{\circ} \mathrm{C}$ )

Hardness as $\mathrm{CaCO} 3$

( $\mathrm{Ca}, \mathrm{Mg}$ )

Noncarbonate hardness as $\mathrm{CaCO} 3$

Percent sodium

Alkalinity as $\mathrm{CaCO} 3$

Specific conductance

(umhos $/ \mathrm{cm}$ at $25^{\circ} \mathrm{C}$ )

$\mathrm{pH}$ (units)

Temperature $\left({ }^{\circ} \mathrm{C}\right)$

Color (Pt-Co units)

Turbidity (NTU)
(1)

177

110

26

19

80

285.

7.9

27.0

22

0

ANALYSIS OF SELECTED TRACE ELEMENTS

(Total concentrations in micrograms per liter, except as indicated)

$\begin{array}{lr}\text { Arsenic (As) } & 0 \\ \text { Barium (Ba) } & - \\ \text { Cadmium (Cd) } & 1 \\ \text { Chromium (Cr) } & 20 \\ \text { Copper (Cu) } & 10 \\ \text { Iron (Fe) } & 100 \\ \text { Lead ( } \mathrm{Pb}) & 19\end{array}$

Manganese (Mn)

Mercury ( $\mathrm{Hg}$ )

Selenium (Se)

Silver ( $\mathrm{Ag})$

Strontium, dissolved ( $\mathrm{Sr}$ ) 290

Zinc $(\mathrm{Zn})$
10

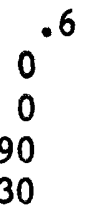

ANALYSIS OF SELECTED PESTICIDE AND INDUSTRIAL COMPOUNDS

( Total concentrations in micrograms per liter)

$\begin{array}{llll}\text { Aldrin } & .00 & \text { Lindane } & .00 \\ \text { Chlordane } & .0 & \text { Methoxych1or } & .00 \\ \text { DDD } & .00 & \text { Mirex } & .00 \\ \text { DDE } & .00 & \text { PCB } & .0 \\ \text { DDT } & .00 & \text { PCN } & .00 \\ \text { Dieldrin } & .00 & \text { Silvex } & .00 \\ \text { Endrin } & .00 & \text { Toxaphene } & 0 \\ \text { Heptachlor } & .00 & 2,4-D & .00 \\ \text { Heptachlor epoxide } & .00 & 2,4,5-T & .00\end{array}$

ANALYSIS OF SELECTED GROSS RADIOACTIVITY

(Total activity concentrations in picocuries per liter)

Gross Alpha, as

uranium natural --

Gross Beta, as

cesium-137
Gross Beta, as

strontium-90 
PUBLIC WATER SUPPLY: Delmar Corporation

COUNTY: Pasco

COLLECTION DATE: 11-22-77

SAMPLING POINT (1) Treated water-282120082391109, tap on holding tank near well number 2.

(2) Raw water-

ANALYSIS OF MAJOR CHEMICAL CONSTITUENTS AND PHYSICAL PROPERTIES

(Dissolved concentrations in milligrams per liter, except as indicated)

$\begin{array}{lc}\text { Silica ( } & 7.1 \\ \text { Calcium (Ca) } & 57 \\ \text { Magnesium (Mg) } & 3.0 \\ \text { Sodium (Na) } & 17 \\ \text { Potassium (K) } & .7 \\ \text { Bicarbonate (HCO3) } & 150 \\ \text { Carbonate (CO3) } & 0 \\ \text { Sulfate (SO4) } & 15 \\ \text { Chloride (C1) } & 34 \\ \text { Fluoride (F) } & .0 \\ \text { Nitrate (NO3-N), } & \\ \quad \text { total (NO2-N), } & .81 \\ \text { Nitrite } \quad & \\ \quad \text { total }\end{array}$
(2)

\section{Dissolved solids}

(residue at $180^{\circ} \mathrm{C}$ )

(1)

\section{9}

Hardness as $\mathrm{CaCO} 3$

( $\mathrm{Ca}, \mathrm{Mg}$ )

160

Noncarbonate hardness

$$
\text { as } \mathrm{CaCO} 3
$$

32

Percent sodium

Alkalinity as $\mathrm{CaCO} 3$

Specific conductance

(umhos $/ \mathrm{cm}$ at $25^{\circ} \mathrm{C}$ )

pH (units)

Temperature $\left({ }^{\circ} \mathrm{C}\right)$

Color (Pt-Co units)

Turbidity (NTU)

ANALYSIS OF SELECTED TRACE ELEMENTS

(Total concentrations in micrograms per liter, except as indicated)

$\begin{array}{lrlr}\text { Arsenic (As) } & 2 & \text { Manganese (Mn) } & 0 \\ \text { Barium (Ba) } & - & \text { Mercury (Hg) } & <.5 \\ \text { Cadmium (Cd) } & 0 & \text { Selenium ( Se) } & 0 \\ \text { Chromium (Cr) } & <10 & \text { Silver ( } \mathrm{Ag}) & 0 \\ \text { Copper (Cu) } & 4 & \text { Strontium, dissolved ( Sr) } & 250 \\ \text { Iron (Fe) } & 20 & \text { Zinc (Zn) } & 10 \\ \text { Lead (Pb) } & 0 & & \end{array}$

ANALYSIS OF SELECTED PESTICIDE AND INDUSTRIAL COMPOUNDS (Total concentrations in micrograms per 1iter)

$\begin{array}{llll}\text { Aldrin } & .00 & \text { Lindane } & .00 \\ \text { Chlordane } & .0 & \text { Methoxychlor } & .00 \\ \text { DDD } & .00 & \text { Mirex } & .00 \\ \text { DDE } & .00 & \text { PCB } & .0 \\ \text { DDT } & .00 & \text { PCN } & .00 \\ \text { Dieldrin } & .00 & \text { Silvex } & .00 \\ \text { Endrin } & .00 & \text { Toxaphene } & 0 \\ \text { Heptachlor } & .00 & 2,4-D & .00 \\ \text { Heptachlor epoxide } & .00 & 2,4,5-T & .00\end{array}$

ANALYSIS OF SELECTED GROSS RADIOACTIVITY

(Total activity concentrations in picocuries per liter)

Gross Alpha, as

uranium natural

Gross Beta, as

cesium-137
Gross Beta, as

strontium-90 
PUBLIC WATER SUPPLY: Deltona

COUNTY: Volusia

COLLECTION DATE: 11-02-77

SAMPLING POINT (1) Treated water -285348081140801 , tap at water treatment plant (we11 number 9).

(2) Raw water-

ANALYSIS OF MAJOR CHEMICAL CONSTITUENTS AND PHYSICAL PROPERTIES (Dissolved concentrations in milligrams per liter, except as indicated)
(1)
(2)
(1)

$\begin{array}{lc}\text { Silica ( } & 18 \\ \text { Calcium (Ca) } & 22 \\ \text { Magnesium (Mg) } & 11 \\ \text { Sodium (Na) } & 60 \\ \text { Potassium (K) } & 2.1 \\ \text { Bicarbonate (HCO3) } & 38 \\ \text { Carbonate (CO3) } & 0 \\ \text { Sulfate (SO4) } & 7.9 \\ \text { Chloride (C1) } & 140 \\ \text { Fluoride (F) } & .1 \\ \text { Nitrate (NO3-N), } & \\ \quad \text { total (NO2-N), } & .00 \\ \text { Nitrite } \quad & \\ \quad \text { total }\end{array}$

Dissolved solids (residue at $180^{\circ} \mathrm{C}$ ) $\quad 304$

Hardness as $\mathrm{CaCO} 3$

$(\mathrm{Ca}, \mathrm{Mg})$

100

Noncarbonate hardness as $\mathrm{CaCO} 3$

69

Percent sodium $\quad 56$

A1kalinity as $\mathrm{CaCO} 3 \quad 31$

Specific conductance

(umhos $/ \mathrm{cm}$ at $25^{\circ} \mathrm{C}$ )

$\mathrm{pH}$ (units)

Temperature $\left({ }^{\circ} \mathrm{C}\right)$

Color (Pt-Co units)

Turbidity (NTU)

304
00
69
56
31
-
7.0
23.5
9
1

ANALYSIS OF SELECTED TRACE ELEMENTS

( Total concentrations in micrograms per liter, except as indicated)

$\begin{array}{lrlr}\text { Arsenic (As) } & 0 & \text { Manganese (Mn) } & 0 \\ \text { Barium (Ba) } & 0 & \text { Mercury (Hg) } & <.5 \\ \text { Cadmium (Cd) } & 0 & \text { Selenium ( Se) } & 0 \\ \text { Chromium (Cr) } & <10 & \text { Silver (Ag) } & 0 \\ \text { Copper ( Cu) } & 1 & \text { Strontium, dissolved (Sr) } 160 \\ \text { Iron (Fe) } & 30 & \text { Zinc (Zn) } & 30 \\ \text { Lead ( } \mathrm{Pb}) & 6 & & \end{array}$

ANALYSIS OF SELECTED PESTICIDE AND INDUSTRIAL COMPOUNDS

(Total concentrations in micrograms per 1iter)

$\begin{array}{llll}\text { Aldrin } & .00 & \text { Lindane } & .00 \\ \text { Chlordane } & .0 & \text { Methoxych 1or } & .00 \\ \text { DDD } & .00 & \text { Mirex } & .00 \\ \text { DDE } & .00 & \text { PCB } & .0 \\ \text { DDT } & .00 & \text { PCN } & .00 \\ \text { Dieldrin } & .00 & \text { Silvex } & .00 \\ \text { Endrin } & .00 & \text { Toxaphene } & 0 \\ \text { Heptachlor } & .00 & 2,4-D & .00 \\ \text { Heptachlor epoxide } & .00 & 2,4,5-\mathrm{T} & .00\end{array}$

ANALYSIS OF SELECTED GROSS RADIOACTIVITY

(Total activity concentrations in picocuries per liter)

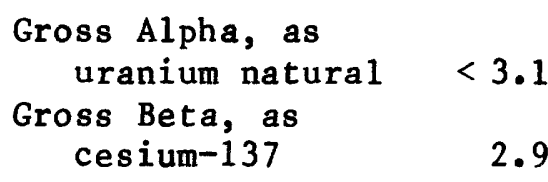

Gross Beta, as

strontium -90

2.6 
PUBLIC WATER SUPPLY: Eaton Park

COUNTY: Polk

COLLECTION DATE: 12-15-77

SAMPLING POINT (1) Treated water-280029081542509, tap outside on south side of pumphouse.

(2) Raw water-

ANALYSIS OF MAJOR CHEMICAL CONSTITUENTS AND PHYSICAL PROPERTIES

(Dissolved concentrations in milligrams per liter, except as indicated)

$\begin{array}{lc}\text { Silica (SiO2) } & (1) \\ \text { Calcium (Ca) } & 21 \\ \text { Magnesium (Mg) } & 36 \\ \text { Sodium (Na) } & 12 \\ \text { Potassium (K) } & 4.5 \\ \text { Bicarbonate (HCO3) } & 160 \\ \text { Carbonate (CO3) } & 0 \\ \text { Sulfate (SO4) } & 3.0 \\ \text { Chloride (C1) } & 9.4 \\ \text { Fluoride (F) } & .2 \\ \text { Nitrate (NO3-N), } & .06 \\ \quad \text { total (NO2-N), } \\ \text { Nitrite } \quad \\ \quad \text { total }\end{array}$

(1) (2)

36

2

.9

0

3.0

9.4

.2

.06

.00
Dissolved solids

(residue at $180^{\circ} \mathrm{C}$ )

(1)

167

Hardness as $\mathrm{CaCO} 3$

( $\mathrm{Ca}, \mathrm{Mg}$ )

140

Noncarbonate hardness as $\mathrm{CaCO} 3$

Percent sodium

Alkalinity as $\mathrm{CaCO} 3$

Specific conductance

(umhos $/ \mathrm{cm}$ at $25^{\circ} \mathrm{C}$ )

$\mathrm{pH}$ (units)

Temperature $\left({ }^{\circ} \mathrm{C}\right)$

Color (Pt-Co units)

Turbidity (NTU)

ANALYSIS OF SELECTED TRACE ELEMENTS

(Total concentrations in micrograms per liter, except as indicated)

$\begin{array}{lrlc}\text { Arsenic (As) } & 1 & \text { Manganese (Mn) } & 0 \\ \text { Barium (Ba) } & -- & \text { Mercury (Hg) } & <.5 \\ \text { Cadmium (Cd) } & 0 & \text { Selenium ( Se) } & 0 \\ \text { Chromium (Cr) } & <10 & \text { Silver (Ag) } & 0 \\ \text { Copper (Cu) } & 8 & \text { Strontium, dissolved (Sr) } & 60 \\ \text { Iron (Fe) } & 240 & \text { Zinc (Zn) } & 10 \\ \text { Lead (Pb) } & 9 & & \end{array}$

ANALYSIS OF SELECTED PESTICIDE AND INDUSTRIAL COMPOUNDS

(Total concentrations in micrograms per 1iter)

$\begin{array}{llll}\text { Aldrin } & .00 & \text { Lindane } & .00 \\ \text { Chlordane } & .0 & \text { Methoxych lor } & .00 \\ \text { DDD } & .00 & \text { Mirex } & .00 \\ \text { DDE } & .00 & \text { PCB } & .0 \\ \text { DDT } & .00 & \text { PCN } & .00 \\ \text { Dieldrin } & .00 & \text { Silvex } & .00 \\ \text { Endrin } & .00 & \text { Toxaphene } & 0 \\ \text { Heptachlor } & .00 & 2,4-D & .00 \\ \text { Heptachlor epoxide } & .00 & 2,4,5-T & .00\end{array}$

ANALYSIS OF SELECTED GROSS RADIOACTIVITY

(Total activity concentrations in picocuries per liter)

Gross Alpha, as uranium natural $\quad 3.6$

Gross Beta, as

cesium-137
Grose Beta, as

strontium -90 
PUBLIC WATER SUPPLY: Englewood

COUNTY: Sarasota

COLLECTION DATE: 12-22-77

SAMPLING POINT (1) Treated water-2657130822-5609, tap outside water treatment plant.

(2) Raw water-

ANALYSIS OF MAJOR CHEMICAL CONSTITUENTS AND PHYSICAL PROPERTIES

(Dissolved concentrations in milligrams per liter, except as indicated)

(1)

Silica ( $\mathrm{SiO} 2)$
Calcium ( $\mathrm{Ca})$
Magnesium (Mg)
Sodium ( Na)
Potassium (K)
Bicarbonate (HCO3)
Carbonate (CO3)
Sulfate ( $\mathrm{SO} 4)$
Chloride (C1)
Fluoride (F)
Nitrate (NO3-N),
total
Nitrite (NO2-N),
total

(2)

$$
\begin{aligned}
& \text { Dissolved solids } \\
& \text { (residue at } 180^{\circ} \mathrm{C} \text { ) } \\
& \text { Hardness as } \mathrm{CaCO} 3 \\
& \text { ( } \mathrm{Ca}, \mathrm{Mg} \text { ) } \\
& \text { Noncarbonate hardness } \\
& \text { as CaCO3 } \\
& \text { Percent sodium } \\
& \text { Alkalinity as CaC03 } \\
& \text { Specific conductance } \\
& \quad \text { (umbos/cm at } 25^{\circ} \mathrm{C} \text { ) } \\
& \text { pH (units) } \\
& \text { Temperature ( }{ }^{\circ} \mathrm{C} \text { ) } \\
& \text { Color (Pt-Co units) } \\
& \text { Turbidity (NTU) }
\end{aligned}
$$

ANALYSIS OF SELECTED TRACE ELEMENTS

(Total concentrations in micrograms per liter, except as indicated)

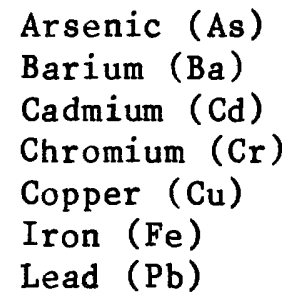

ANALYSIS OF SELECTED PESTICIDE AND INDUSTRIAL COMPOUNDS

(Total concentrations in micrograms per liter)
Aldrin
Chlordane
DDD
DDE
DDT
Dieldrin
Endrin
Heptach lor
Heptachlor epoxide

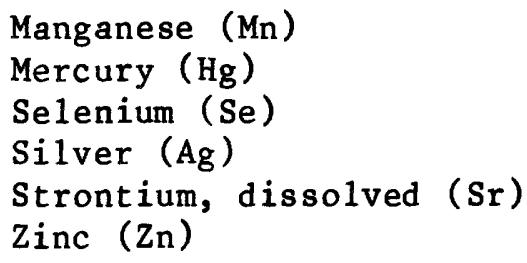

(1)

8.6
Lindane
Methoxychlor
Mirex
PCB
PCN

Silvex

Toxaphene

2, 4-D

$2,4,5-T$

ANALYSIS OF SELECTED GROSS RADIOACTIVITY

(Total activity concentrations in picocuries per liter)

$\begin{array}{ll}\text { Gross Alpha, as } & \\ \text { uranium natural } & <3.9 \\ \text { Gross Beta, as } & <1.4 \\ \text { cesium-137 } & <\end{array}$

Gross Beta, as

strontium -90

cesium-137 $\quad<1.4$ 
PUBLIC WATER SUPPLY: EUstis

COLLECTION DATE: 11-16-77

SAMPLING POINT (1) Treated water-285047081401101, tap inside pumphouse.

(2) Raw water-

ANALYSIS OF MAJOR CHEMICAL CONSTITUENTS AND PHYSICAL PROPERTIES

(Dissolved concentrations in milligrams per liter, except as indicated)

(1) (2)

(1)

$\begin{array}{lc}\text { Silica (SiO2) } & 11 \\ \text { Calcium ( } \mathrm{Ca}) & 24 \\ \text { Magnesium (Mg) } & 8.7 \\ \text { Sodium (Na) } & 5.8 \\ \text { Potassium (K) } & 1.1 \\ \text { Bicarbonate (HCO3) } & 100 \\ \text { Carbonate (CO3) } & 0 \\ \text { Sulfate (SO4) } & 5.5 \\ \text { Chloride (C1) } & 14 \\ \text { Fluoride (F) } & .1 \\ \text { Nitrate (NO3-N), } & \\ \quad \text { total (NO2-N), } \\ \text { Nitrite } \quad \\ \quad \text { total }\end{array}$

Dissolved solids (residue at $180^{\circ} \mathrm{C}$ ) $\quad 114$

Hardness as $\mathrm{CaCO}$

$(\mathrm{Ca}, \mathrm{Mg})$

96

Noncarbonate hardness

$38 \mathrm{CaCO} 3$

14

Percent sodium 12

Alkalinity as $\mathrm{CaCO} 3 \quad 82$

Specific conductance

(umhos $/ \mathrm{cm}$ at $25^{\circ} \mathrm{C}$ )

$\mathrm{pH}$ (units)

Temperature $\left({ }^{\circ} \mathrm{C}\right)$

Color (Pt-Co units)

Turbidity (NTU)

14
96
14
12
82
--
7.2
24.0
2
0

ANALYSIS OF SELECTED TRACE ELEMENTS

(Total concentrations in micrograms per liter, except as indicated)

$\begin{array}{lr}\text { Arsenic ( } \mathrm{As} \text { ) } & 0 \\ \text { Barium ( } \mathrm{Ba}) & 0 \\ \text { Cadmium (Cd) } & 1 \\ \text { Chromium (Cr) } & 10 \\ \text { Copper (Cu) } & 5 \\ \text { Iron (Fe) } & 50 \\ \text { Lead (Pb) } & 5\end{array}$

Manganese $(M n)$

Mercury $(\mathrm{Hg})$

Selenium ( $\mathrm{Se}$ )

10

Silver ( $\mathrm{Ag}$ )

1.7

1

5 Strontium, dissolved ( $\mathrm{Sr}$ ) 140

5

Zinc $(\mathrm{Zn})$

60

ANALYSIS OF SELECTED PESTICIDE AND INDUSTRIAL COMPOUNDS

(Total concentrations in micrograms per 1iter)

$\begin{array}{llll}\text { Aldrin } & .00 & \text { Lindane } & .00 \\ \text { Chlordane } & .0 & \text { Methoxych lor } & .00 \\ \text { DDD } & .00 & \text { Mirex } & .00 \\ \text { DDE } & .00 & \text { PCB } & .0 \\ \text { DDT } & .00 & \text { PCN } & .00 \\ \text { Dieldrin } & .00 & \text { Silvex } & .00 \\ \text { Endrin } & .00 & \text { Toxaphene } & 0 \\ \text { Heptachlor } & .00 & 2,4-D & .00 \\ \text { Heptachlor epoxide } & .00 & 2,4,5-\mathrm{T} & .00\end{array}$

ANALYSIS OF SELECTED GROSS RADIOACTIVITY

(Total activity concentrations in picocuries per liter)

Gross Alpha, as uranium natural

Gross Beta, as

cesium-137
2.4

Gross Beta, as

strontium-90
.00

.00

.00

.0

.00

.00

.00 
PUBLIC WATER SUPPLY: Fernandina Beach

COUNTY: Nassau

COLLECTION DATE: $12-08-77$

SAMPLING POINT (1) Treated water-303701081263101, fire hydrant at the Buccaneer Trail (Route A1A), 1.5 miles south of Sadler Road.

(2) Raw water-

ANALYSIS OF MAJOR CHEMICAL CONSTITUENTS AND PHYSICAL PROPERTIES

(Dissolved concentrations in milligrams per liter, except as indicated)

Silica (SiO2)

(1)

(2)

Calcium ( $\mathrm{Ca}$ )

Magnesium ( $\mathrm{Mg})$

Sodium ( $\mathrm{Na}$ )

Potassium (K)

Bicarbonate (HCO3)

Carbonate ( $\mathrm{CO} 3$ )

Sulfate ( 504 )

Chloride (C1)

Fluoride (F)

Nitrate (NO3-N), total

Nitrite $(\mathrm{NO2}-\mathrm{N})$, total

Dissolved solids

(1)

(residue at $180^{\circ} \mathrm{C}$ )

Hardness as $\mathrm{CaCO}_{3}$

$(\mathrm{Ca}, \mathrm{Mg})$

Noncarbonate hardness

as $\mathrm{CaCO} 3$

Percent sodium

Alkalinity as $\mathrm{CaCO} 3$

Specific conductance

(umhos $/ \mathrm{cm}$ at $25^{\circ} \mathrm{C}$ )

$\mathrm{pH}$ (units)

715

Temperature $\left({ }^{\circ} \mathrm{C}\right)$

7.2

Color (Pt-Co units)

Turbidity (NTU)

ANALYSIS OF SELECTED TRACE ELEMENTS

(Total concentrations in micrograms per liter, except as indicated)

Arsenic (As)

Barium ( $\mathrm{Ba}$ )

Cadmium ( $C d)$

Chromium (Cr)

Copper (Cu)

Iron (Fe)

Lead $(\mathrm{Pb})$
Manganese (Mn)

Mercury $(\mathrm{Hg})$

Selenium ( $\mathrm{Se}$ )

Silver ( $\mathrm{Ag}$ )

Strontium, dissolved $(S r)$

Zinc $(\mathrm{Zn})$

ANALYSIS OF SELECTED PESTICIDE AND INDUSTRIAL COMPOUNDS

(Total concentrations in micrograms per 1iter)
Aldrin
Chlordane
DDD
DDE
DDT
Dieldrin
Endrin
Heptach lor
Heptachlor epoxide

Lindane
Methoxych lor
Mirex
PCB
PCN
Silvex
Toxaphene
$2,4-D$
$2,4,5-T$

ANALYSIS OF SELECTED GROSS RADIOACTIVITY

(Total activity concentrations in picocuries per liter)

Gross Alpha, as

uranium natural $\quad<4.4$

Gross Beta, as

cesium-137
Gross Beta, as

strontium-90

2.2 
PUBLIC WATER SUPPLY: First Florida Utilities

COUNTY: Palm Beach

COLLECTION DATE: 11-30-77

SAMPLING POINT (1) Treated water-264147080084002, tap at Shadow Lake Mobile Home Village at southeast corner (2" pipe, $4^{\prime}$ high).

(2) Raw water-

ANALYSIS OF MAJOR CHEMICAL CONSTITUENTS AND PHYSICAL PROPERTIES (Dissolved concentrations in milligrams per liter, except as indicated)

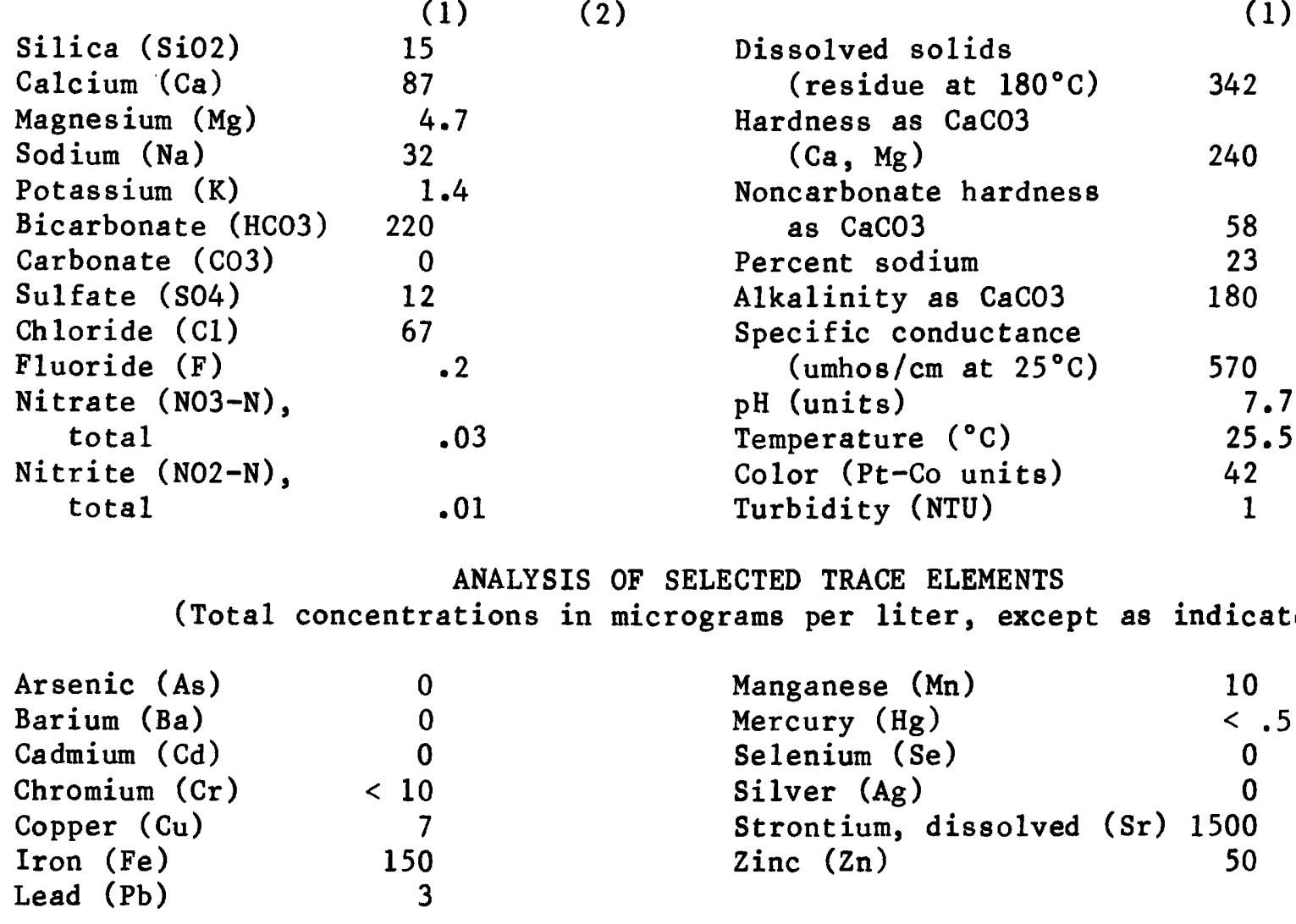

ANALYSIS OF SELECTED PESTICIDE AND INDUSTRIAL COMPOUNDS

(Total concentrations in micrograms per liter)

$\begin{array}{llll}\text { Aldrin } & .00 & \text { Lindane } & .00 \\ \text { Chlordane } & .0 & \text { Methoxych 1or } & .00 \\ \text { DDD } & .00 & \text { Mirex } & .00 \\ \text { DDE } & .00 & \text { PCB } & .0 \\ \text { DDT } & .00 & \text { PCN } & .00 \\ \text { Dieldrin } & .00 & \text { Silvex } & .00 \\ \text { Endrin } & .00 & \text { Toxaphene } & 0 \\ \text { Heptachlor } & .00 & 2,4-D & .02 \\ \text { Heptachlor epoxide } & .00 & 2,4,5-T & .00\end{array}$

ANALYSIS OF SELECTED GROSS RADIOACTIVITY

(Total activity concentrations in picocuries per liter)

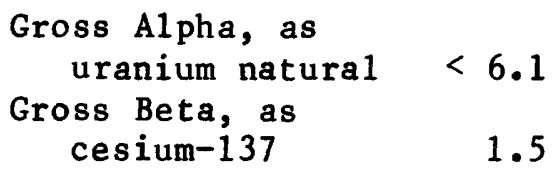

Gross Beta, as

strontium-90

1.3 
PUBLIC WATER SUPPLY: Flagler Beach

COUNTY: Flagler COLLECTION DATE: 11-09-77

SAMPLING POINT (1) Treated water-292830081114601, tap inside water treatment plant.

(2) Raw water-

ANALYSIS OF MAJOR CHEMICAL CONSTITUENTS AND PHYSICAL PROPERTIES (Dissolved concentrations in milligrams per liter, except as indicated)

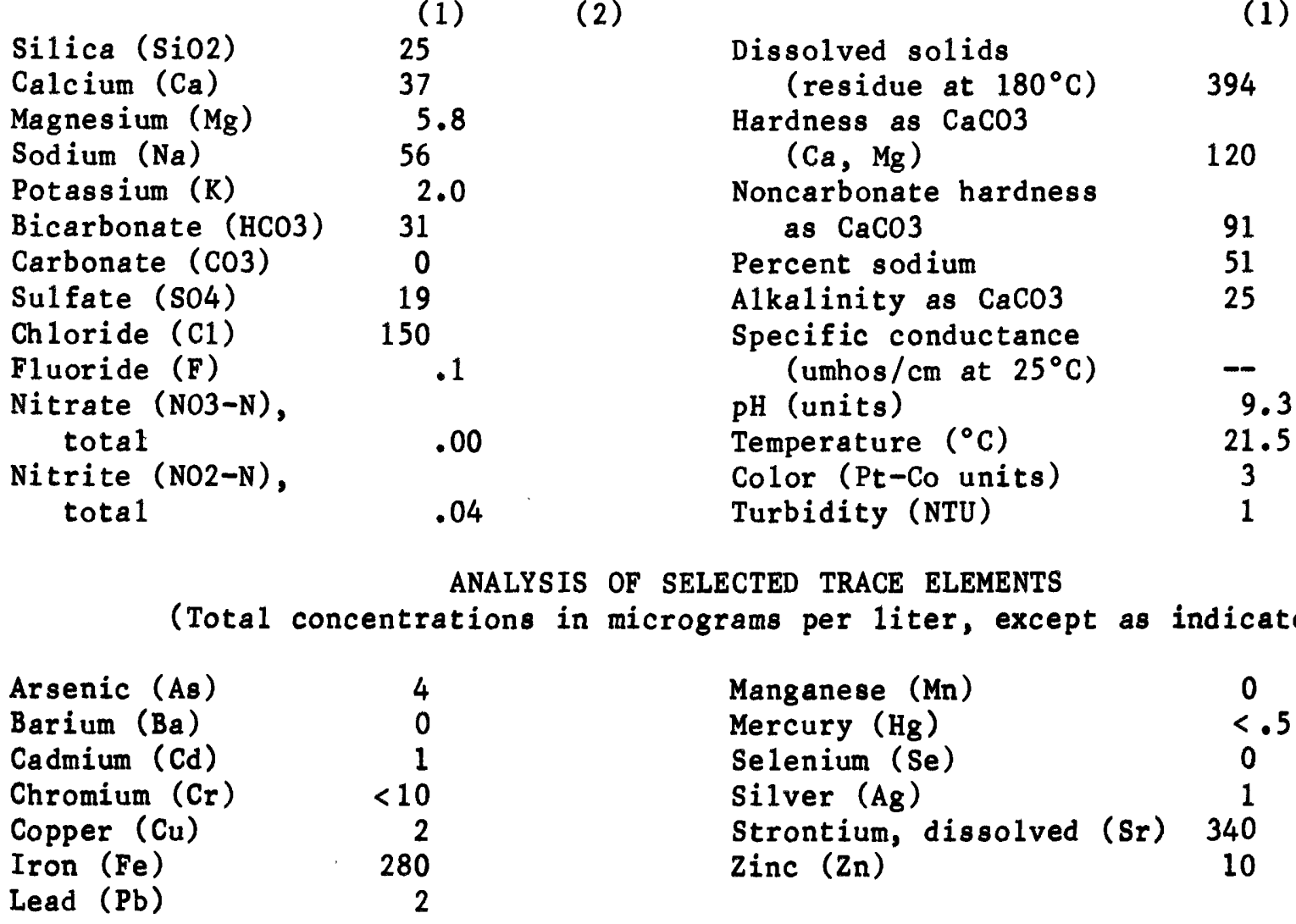

ANALYSIS OF SELECTED PESTICIDE AND INDUSTRIAL COMPOUNDS

(Total concentrations in micrograms per liter)

$\begin{array}{llll}\text { Aldrin } & .00 & \text { Lindane } & .00 \\ \text { Chlordane } & .0 & \text { Methoxychlor } & .00 \\ \text { DDD } & .00 & \text { Mirex } & .00 \\ \text { DDE } & .00 & \text { PCB } & .0 \\ \text { DDT } & .00 & \text { PCN } & .00 \\ \text { Dieldrin } & .00 & \text { Silvex } & .00 \\ \text { Endrin } & .00 & \text { Toxaphene } & 0 \\ \text { Heptachlor } & .00 & 2,4-D & .00 \\ \text { Heptachlor epoxide } & .00 & 2,4,5-T & .00\end{array}$

ANALYSIS OF SELECTED GROSS RADIOACTIVITY

(Total activity concentrations in picocuries per liter)

Gross Alpha, as uranium natural $<2.7$

Gross Beta, as

cesium- 137
Gross Beta, as

strontium -90
(1)

394

20

1

25

9.3

1.5

3 
PUBLIC WATER SUPPLY: F1orida City

COUNTY: Dade

COLLECTION DATE: 12-15-77

SAMPLING POINT (1) Treated water-252632080285501, tap at house, 351 SW 5th Street, Florida City.

(2) Raw water-

ANALYSIS OF MAJOR CHEMICAL CONSTITUENTS AND PHYSICAL PROPERTIES (Dissolved concentrations in milligrams per liter, except as indicated)
(1)
(2)
(1)

$\begin{array}{lc}\text { Silica (SiO2) } & 3.6 \\ \text { Calcium (Ca) } & 88 \\ \text { Magnesium (Mg) } & 3.3 \\ \text { Sodium (Na) } & 27 \\ \text { Potassium (K) } & 4.1 \\ \text { Bicarbonate (HCO3) } & 260 \\ \text { Carbonate (CO3) } & 0 \\ \text { Sulfate (SO4) } & 36 \\ \text { Chloride (C1) } & 31 \\ \text { Fluoride (F) } & .1 \\ \text { Nitrate (NO3-N), } & \\ \quad \text { total (NO2-N), } & .15 \\ \text { Nitrite (NO2-N) } \\ \quad \text { total }\end{array}$

Dissolved solids (residue at $180^{\circ} \mathrm{C}$ )

319

Hardness as $\mathrm{CaCO} 3$

$(\mathrm{Ca}, \mathrm{Mg})$

230

Noncarbonate hardness as $\mathrm{CaCO} 3$

21

Percent sodium

20

Alkalinity as $\mathrm{CaCO} 3 \quad 210$

.1

Specific conductance

(umhos/cm at $25^{\circ} \mathrm{C}$ )

545

$\mathrm{pH}$ (units)

Temperature $\left({ }^{\circ} \mathrm{C}\right)$

Color (Pt-Co units)

25.0

.00

Turbidity (NTU)

ANALYSIS OF SELECTED TRACE ELEMENTS

(Total concentrations in micrograms per liter, except as indicated)

$\begin{array}{lrlr}\text { Arsenic (As) } & 2 & \text { Manganese (Mn) } & 0 \\ \text { Barium (Ba) } & -- & \text { Mercury (Hg) } & .6 \\ \text { Cadmium (Cd) } & 4 & \text { Selenium (Se) } & 0 \\ \text { Chromium (Cr) } & 30 & \text { Silver (Ag) } & 1 \\ \text { Copper (Cu) } & 42 & \text { Strontium, dissolved ( } \mathrm{Sr}) & 830 \\ \text { Iron (Fe) } & 50 & \text { Zinc ( } \mathrm{Cn}) & 10 \\ \text { Lead (Pb) } & 43 & & \end{array}$

ANALYSIS OF SELECTED PESTICIDE AND INDUSTRIAL COMPOUNDS (Total concentrations in micrograms per 1 iter)

$\begin{array}{llll}\text { Aldrin } & .00 & \text { Lindane } & .00 \\ \text { Chlordane } & .0 & \text { Methoxychlor } & .00 \\ \text { DDD } & .00 & \text { Mirex } & .00 \\ \text { DDE } & .00 & \text { PCB } & .0 \\ \text { DDT } & .00 & \text { PCN } & .00 \\ \text { Dieldrin } & .00 & \text { Silvex } & .00 \\ \text { Endrin } & .00 & \text { Toxaphene } & 0 \\ \text { Heptach1or } & .00 & 2,4-D & .00 \\ \text { Heptachlor epoxide } & .00 & 2,4,5-T & .00\end{array}$

ANALYSIS OF SELECTED GROSS RADIOACTIVITY

(Total activity concentrations in picocuries per liter)

Gross Alpha, as

uranium natural $<4.2$

Gross Beta, as

cesium-137
Gross Beta, as

strontium-90

4.3 
PUBLIC WATER SUPPLY: Forest Hills

COUNTY: Pasco

COLLECTION DATE: 12-20-77

SAMPLING POINT (1) Treated water-281041082435309, tap inside pumphouse building (well number 2).

(2) Raw water-

ANALYSIS OF MAJOR CHEMICAL CONSTITUENTS AND PHYSICAL PROPERTIES

(Dissolved concentrations in milligrams per liter, except as indicated)

Silica (SiO2)

Calcium ( $\mathrm{Ca}$ )

Magnesium ( $\mathrm{Mg}$ )

Sodium ( $\mathrm{Na}$ )

Potassium (K)

Bicarbonate ( $\mathrm{HCO} 3)$

Carbonate ( $\mathrm{CO} 3)$

Sulfate (S04)

Chloride (C1)

Fluoride (F)

Nitrate (NO3-N), total

Nitrite (NO2-N), total
(1)

9.0

(2)

68

4.1

46

1.7

120

0

34

96

.0

5.8

.00
Dissolved solids

(residue at $180^{\circ} \mathrm{C}$ ) 321

Hardness as $\mathrm{CaCO} 3$

$(\mathrm{Ca}, \mathrm{Mg}$ )

190

Noncarbonate hardness as $\mathrm{CaCO} 3$

88

Percent sodium

Alkalinity as $\mathrm{CaCO} 3$

Specific conductance

(umhos $/ \mathrm{cm}$ at $25^{\circ} \mathrm{C}$ )

$\mathrm{pH}$ (units)

Temperature $\left({ }^{\circ} \mathrm{C}\right)$

Color ( $\mathrm{Pt}-\mathrm{Co}$ units)

Turbidity (NTU)
(1)

ANALYSIS OF SELECTED TRACE ELEMENTS

(Total concentrations in micrograms per liter, except as indicated)

$\begin{array}{lrlc}\text { Arsenic (As) } & 1 & \text { Manganese (Mn) } & 10 \\ \text { Barium (Ba) } & - & \text { Mercury (Hg) } & <.5 \\ \text { Cadmium (Cd) } & 0 & \text { Selenium (Se) } & 4 \\ \text { Chromium (Cr) } & <10 & \text { Silver (Ag) } & 0 \\ \text { Copper (Cu) } & 3 & \text { Strontium, dissolved (Sr) } & 110 \\ \text { Iron (Fe) } & 70 & \text { Zinc ( } \mathrm{nn} \text { ) } & 20 \\ \text { Lead (Pb) } & 0 & & \end{array}$

ANALYSIS OF SELECTED PESTICIDE AND INDUSTRIAL COMPOUNDS

(Total concentrations in micrograms per liter)

$\begin{array}{llll}\text { Aldrin } & - & \text { Lindane } & - \\ \text { Chlordane } & -- & \text { Methoxychlor } \\ \text { DDD } & -- & \text { Mirex } & - \\ \text { DDE } & -- & \text { PCB } & - \\ \text { DDT } & -- & \text { PCN } & - \\ \text { Dieldrin } & -- & \text { Silvex } & - \\ \text { Endrin } & -- & \text { Toxaphene } & - \\ \text { Heptachlor } & -- & 2,4-D & - \\ \text { Heptachlor epoxide } & - & 2,4,5-T & -\end{array}$

ANALYSIS OF SELECTED GROSS RADIOACTIVITY

(Total activity concentrations in picocuries per liter)

Gross Alpha, as uranium natural

Gross Beta, as

cesium-137
Gross Beta, as

strontium-90

2.8 
PUBLIC WATER SUPPLY: Fort Meade

COUNTY: Polk

COLLECTION DATE: 12-15-77

SAMPLING POINT (1) Treated water-274512082483509, tap in main feeder 1 ine on north side of pump house building.

(2) Raw water-

ANALYSIS OF MAJOR CHEMICAL CONSTITUENTS AND PHYSICAL PROPERTIES (Dissolved concentrations in milligrams per liter, except as indicated)

(1) (2)

$\begin{array}{lc}\text { Silica (SiO2) } & 20 \\ \text { Calcium (Ca) } & 55 \\ \text { Magnesium (Mg) } & 17 \\ \text { Sodium ( } \mathrm{Na}) & 7.3 \\ \text { Potassium (K) } & 1.6 \\ \text { Bicarbonate (HCO3) } & 160 \\ \text { Carbonate (CO3) } & 0 \\ \text { Sulfate (SO4) } & 68 \\ \text { Chloride (C1) } & 12 \\ \text { Fluoride (F) } & .4 \\ \text { Nitrate (NO3-N), } & \\ \quad \text { total (NO2-N), } & .02 \\ \text { Nitrite } \quad & \\ \quad \text { total }\end{array}$

(1)

\section{ANALYSIS OF SELECTED TRACE ELEMENTS}

(Total concentrations in micrograms per liter, except as indicated)

$\begin{array}{lr}\text { Arsenic ( } \mathrm{As}) & - \\ \text { Barium ( } \mathrm{Ba}) & - \\ \text { Cadmium ( } \mathrm{Cd}) & 0 \\ \text { Chromium ( } \mathrm{Cr} \text { ) } & <10 \\ \text { Copper ( } \mathrm{Cu}) & 13 \\ \text { Iron ( } \mathrm{Fe}) & 10 \\ \text { Lead ( } \mathrm{Pb}) & 8\end{array}$

$\begin{array}{lr}\text { Manganese (Mn) } & 1 \\ \text { Mercury ( } \mathrm{Hg}) & <.5 \\ \text { Selenium ( } \mathrm{Se}) & - \\ \text { Silver ( } \mathrm{Ag}) & 0 \\ \text { Strontium, dissolved } & (\mathrm{Sr}) 2,300 \\ \text { Zinc ( } \mathrm{Zn}) & 10\end{array}$

ANALYSIS OF SELECTED PESTICIDE AND INDUSTRIAL COMPOUNDS

(Total concentrations in micrograms per liter)

$\begin{array}{llll}\text { Aldrin } & .00 & \text { Lindane } & .00 \\ \text { Chlordane } & .0 & \text { Methoxych lor } & .00 \\ \text { DDD } & .00 & \text { Mirex } & .00 \\ \text { DDE } & .00 & \text { PCB } & .0 \\ \text { DDT } & .00 & \text { PCN } & .00 \\ \text { Dieldrin } & .00 & \text { Silvex } & .00 \\ \text { Endrin } & .00 & \text { Toxaphene } & 0 \\ \text { Heptachlor } & .00 & 2,4-D & .00 \\ \text { Heptachlor epoxide } & .00 & 2,4,5-T & .00\end{array}$

ANALYSIS OF SELECTED GROSS RADIOACTIVITY

(Total activity concentrations in picocuries per liter)

Gross Alpha, as

uranium natural

Gross Beta, as

Ces 1um-137
Gross Beta, as

strontium-90 
PUBLIC WATER SUPPLY: Frostproof

COUNTY: Polk

COLLECTION DATE: 12-21-77

SAMPLING POINT (1) Treated water-274443081314709, tap on south side of pumphouse building.

(2) Raw water-

ANALYSIS OF MAJOR CHEMICAL CONSTITUENTS AND PHYSICAL PROPERTIES (Dissolved concentrations in milligrams per liter, except as indicated)

(1)

\begin{tabular}{lc} 
Silica ( SiO2) & 24 \\
Calcium (Ca) & 32 \\
Magnesium (Mg) & 16 \\
Sodium (Na) & 8.2 \\
Potassium (K) & .7 \\
Bicarbonate (HCO3) & 160 \\
Carbonate (CO3) & 0 \\
Sulfate (SO4) & \multicolumn{2}{c}{8.2} \\
Chloride (C1) & 15 \\
Fluoride (F) & \multicolumn{2}{c}{.2} \\
Nitrate (NO3-N), & \\
$\quad$ total (NO2-N), & .02 \\
Nitrite (NO2) \\
$\quad$ total
\end{tabular}

(2)

Dissolved solids

$$
\text { (residue at } 180^{\circ} \mathrm{C} \text { ) } \quad 174
$$

Hardness as $\mathrm{CaCO} 3$ (Ca, Mg) 150

Noncarbonate hardness as $\mathrm{CaCO} 3$

(1)

ANALYSIS OF SELECTED TRACE ELEMENTS

(Total concentrations in micrograms per liter, except as indicated)

$\begin{array}{lr}\text { Arsenic (As) } & 0 \\ \text { Barium (Ba) } & - \\ \text { Cadmium (Cd) } & 0 \\ \text { Chromium (Cr) } & 20 \\ \text { Copper (Cu) } & 4 \\ \text { Iron (Fe) } & 20 \\ \text { Lead ( } \mathrm{Pb} \text { ) } & 0\end{array}$

Manganese (Mn)

10

Mercury ( $\mathrm{Hg})$

$<.5$

Selenium ( $\mathrm{Se}$ )

Silver (Ag)

Alkalinity as $\mathrm{CaCO} 3 \quad 130$

Specific conductance

$\mathrm{pH}$ (units)

Temperature $\left({ }^{\circ} \mathrm{C}\right)$

Color (Pt-Co units)

2

1

ANALYSIS OF SELECTED PESTICIDE AND INDUSTRIAL COMPOUNDS

(Total concentrations in micrograms per liter)

$\begin{array}{llll}\text { Aldrin } & .00 & \text { Lindane } & .00 \\ \text { Chlordane } & .0 & \text { Methoxych lor } & .00 \\ \text { DDD } & .00 & \text { Mirex } & .00 \\ \text { DDE } & .00 & \text { PCB } & .0 \\ \text { DDT } & .00 & \text { PCN } & .00 \\ \text { Dieldrin } & .00 & \text { Silvex } & .00 \\ \text { Endrin } & .00 & \text { Toxaphene } & 0 \\ \text { Heptachlor } & .00 & 2,4-D & .00 \\ \text { Heptachlor epoxide } & .00 & 2,4,5-\mathrm{T} & .00\end{array}$

ANALYSIS OF SELECTED GROSS RADIOACTIVITY

(Total activity concentrations in picocuries per liter)

Gross Alpha, as uranium natura1 12

Gross Beta, as cesium-137
Gross Beta, as

strontium-90 
PUBLIS WATER SUPPLY: G. Ho Woot Memorial Hospital

COUNTY: DeSoto COLLECTION DATE: 12-15-77

SANPIING POTNT (1) Treated gater-270825081482109, tap at south side of i.

(2) (ia \%

ANALYSIS OF WJU WEICAL CONSTITUENTS AND PHYSICAL PROPERTIES

(Dissolved concentrations in milligrams per liter, except as indicated)

(1) (2)

$\begin{array}{lc}\text { Silica (SiO2) } & 13 \\ \text { Calcium (Ca) } & 30 \\ \text { Magnesium (Mg) } & 24 \\ \text { Sotiur (Na) } & 110 \\ \text { Potassium (K) } & 3.3 \\ \text { Bicarbonate (HCO3) } & 79 \\ \text { Carbonate (CO3) } & 1 \\ \text { Sulfate (SO4) } & 260 \\ \text { Chloride (C1) } & 74 \\ \text { Fluoride (F) } & .8 \\ \text { Nitrate (NO3-N), } & \\ \quad \text { total. } & .07 \\ \text { Nitrite (NO2-N), } \\ \quad \text { total }\end{array}$

Dissolved solids

(1)

(2)

(residue at $180^{\circ} \mathrm{C}$ ) $\quad 544$

Hardness as $\mathrm{CaCO}$

$(\mathrm{Ca}, \mathrm{Mg})$

190

Noncarbonate hardness

as $\mathrm{CaCO} 3 \quad 120$

Percent sodium 57

Alkalinity as $\mathrm{CaCO} 3 \quad 66$

Specific conductance

(umhos $/ \mathrm{cm}$ at $25^{\circ} \mathrm{C}$ )

$\mathrm{pH}$ (units)

Temperature $\left({ }^{\circ} \mathrm{C}\right)$

Color (Pt-Co units)

Turbidity (NTU)

ANALYSIS OF SELECTED TRACE ELEMENTS

(Total concentrations in micrograms per liter, except as indicated)

$\begin{array}{lrlr}\text { Arsenic (As) } & 0 & \text { Manganese (Mn) } & 0 \\ \text { Barium (Ba) } & - & \text { Mercury (Hg) } & <.5 \\ \text { Cadmium (Cd) } & 0 & \text { Selenium (Se) } & 0 \\ \text { Chromium (Cr) } & <10 & \text { Silver (Ag) } & 0 \\ \text { Copper (Cu) } & 3 & \text { Strontium, dissolved (Sr) 12000 } \\ \text { Iron (Fe) } & 0 & \text { Zinc (Zn) } & 0 \\ \text { Lead (Pb) } & 8 & & \end{array}$

ANALYSIS OF SELECTED PESTICIDE AND INDUSTRIAL COMPOUNDS

(Total concentrations in micrograms per 1iter)

$\begin{array}{llll}\text { Aldrin } & .00 & \text { Lindane } & .00 \\ \text { Chlordane } & .0 & \text { Methoxych1or } & .00 \\ \text { DDD } & .00 & \text { Mirex } & .00 \\ \text { DDE } & .00 & \text { PCB } & .0 \\ \text { DDT } & .00 & \text { PCN } & .00 \\ \text { Dieldrin } & .00 & \text { Silvex } & .00 \\ \text { Endrin } & .00 & \text { Toxaphene } & 0 \\ \text { Heptachlor } & .00 & 2,4-D & .00 \\ \text { Heptachlor epoxide } & .00 & 2,4,5-\mathrm{T} & .00\end{array}$

ANALYSIS OF SELECTED GROSS RADIOACTIVITY

(Total activity concentrations in picocuries per liter)

Gross Alpha, as uranium natural 10

Gross Beta, as

cesium-137
Gross Beta, as

strontium -90
.00

.00

.00

.00

.00

.00

.00 
PUBLIC WATER SUPPLY: Garden Grove

COUNTY: Polk COLLECTION DATE: $12-14-77$

SAMPLING POINT (1) Treated water-280014081414509, tap on holding tank just north of water treatment plant building.

(2) Raw water-

ANALYSIS OF MAJOR CHEMICAL CONSTITUENTS AND PHYSICAL PROPERTIES

(Dissolved concentrations in milligrams per liter, except as indicated)

(1) (2)

$\begin{array}{lc}\text { Silica (SiO2) } & 12 \\ \text { Calcium (Ca) } & 42 \\ \text { Magnesium (Mg) } & 6.0 \\ \text { Sodium (Na) } & 7.2 \\ \text { Potassium (K) } & 1.3 \\ \text { Bicarbonate (HCO3) } & 130 \\ \text { Carbonate (CO3) } & 0 \\ \text { Sulfate (SO4) } & 16 \\ \text { Chloride (C1) } & 15 \\ \text { Fluoride (F) } & .1 \\ \text { Nitrate (NO3-N), } & \\ \quad \text { total (NO2-N), } & .37 \\ \text { Nitrite (Notal } \\ \quad \text { total }\end{array}$

(1)

ANALYSIS OF SELECTED TRACE ELEMENTS

(Total concentrations in micrograms per liter, except as indicated)

$\begin{array}{lrlc}\text { Arsenic (As) } & 3 & \text { Manganese (Mn) } & 0 \\ \text { Barium (Ba) } & - & \text { Mercury (Hg) } & <.5 \\ \text { Cadmium (Cd) } & 0 & \text { Selenium (Se) } & 1 \\ \text { Chromium (Cr) } & 10 & \text { Silver (Ag) } & 0 \\ \text { Copper (Cu) } & 6 & \text { Strontium, dissolved ( } \mathrm{Sr} \text { ) } & 100 \\ \text { Iron (Fe) } & 30 & \text { Zinc ( } \mathrm{Cn} \text { ) } & 10 \\ \text { Lead (Pb) } & 8 & & \end{array}$

ANALYSIS OF SELECTED PESTICIDE AND INDUSTRIAL COMPOUNDS

(Total concentrations in micrograms per liter)

$\begin{array}{llll}\text { Aldrin } & .00 & \text { Lindane } & .00 \\ \text { Chlordane } & .0 & \text { Methoxych1or } & .00 \\ \text { DDD } & .00 & \text { Mirex } & .00 \\ \text { DDE } & .00 & \text { PCB } & .0 \\ \text { DDT } & .00 & \text { PCN } & .00 \\ \text { Dieldrin } & .00 & \text { Silvex } & .00 \\ \text { Endrin } & .00 & \text { Toxaphene } & 0 \\ \text { Heptachlor } & .00 & 2,4-D & .00 \\ \text { Heptachlor epoxide } & .00 & 2,4,5-\mathrm{T} & .00\end{array}$

ANALYSIS OF SELECTED GROSS RADIOACTIVITY

(Total activity concentrations in picocuries per liter)

Gross Alpha, as uranium natural $\quad 6.4$ Gross Beta, as cesium-137
Gross Beta, as strontium -90

4.1 
PUBLIC WATER SUPPLY: Gifford

\section{COUNTY: Indian River}

COLLECTION DATE: 11-09-77

SAMPLING POINT (1) Treated water-274008080274901, tap outside utilities office.

(2) Raw water-

ANALYSIS OF MAJOR CHEMICAL CONSTITUENTS AND PHYSICAL PROPERTIES (Dissolved concentrations in milligrams per liter, except as indicated)

$\begin{array}{lc} & (1) \\ \text { Silica (SiO2) } & 20 \\ \text { Calcium ( Ca) } & 52 \\ \text { Magnesium (Mg) } & 41 \\ \text { Sodium (Na) } & 130 \\ \text { Potassium (K) } & 7.0 \\ \text { Bicarbonate (HCO3) } & 210 \\ \text { Carbonate (CO3) } & 0 \\ \text { Sulfate (SO4) } & 89 \\ \text { Chloride (C1) } & 240 \\ \text { Fluoride (F) } & .8 \\ \text { Nitrate (NO3-N), } & .00 \\ \quad \text { total (NO2-N), } & \\ \text { Nitrite } & \\ \quad \text { total } & \end{array}$
(2)

(1)

Dissolved solids (residue at $180^{\circ} \mathrm{C}$ )

Hardness as $\mathrm{CaCO} 3$ ( $\mathrm{Ca}, \mathrm{Mg}$ )

999

310

Noncarbonate hardness as $\mathrm{CaCO} 3$

Percent sodium

Alkalinity as $\mathrm{CaCO} 3$

Specific conductance (umhos $/ \mathrm{cm}$ at $25^{\circ} \mathrm{C}$ )

$\mathrm{pH}$ (units)

Temperature $\left({ }^{\circ} \mathrm{C}\right)$

Color (Pt-Co units)

Turbidity (TU)
140

48

170

1,260

7.4

24.5

0

1

ANALYSIS OF SELECTED TRACE ELEMENTS

(Total concentrations in micrograms per liter, except as indicated)

$\begin{array}{lrlr}\text { Arsenic (As) } & -- & \text { Manganese (Mn) } & 10 \\ \text { Barium (Ba) } & 0 & \text { Mercury (Hg) } & -- \\ \text { Cadmium ( } \mathrm{Cd}) & 0 & \text { Selenium ( } \mathrm{Se}) & - \\ \text { Chromium (Cr) } & <10 & \text { Silver (Ag) } & 0 \\ \text { Copper (Cu) } & 71 & \text { Strontium, dissolved ( } \mathrm{Sr}) 8,200 \\ \text { Iron (Fe) } & 50 & \text { Zinc (Zn) } & 40 \\ \text { Lead ( } \mathrm{Pb}) & 0 & & \end{array}$

ANALYSIS OF SELECTED PESTICIDE AND INDUSTRIAL COMPOUNDS

(Total concentrations in micrograms per liter)

$\begin{array}{llll}\text { Aldrin } & .00 & \text { Lindane } & .00 \\ \text { Chlordane } & .0 & \text { Methoxychlor } & -- \\ \text { DDD } & .00 & \text { Mirex } & .0 \\ \text { DDE } & .00 & \text { PCB } & .00 \\ \text { DDT } & .00 & \text { PCN } & .00 \\ \text { Dieldrin } & .00 & \text { Silvex } & - \\ \text { Endrin } & .00 & \text { Toxaphene } & .00 \\ \text { Heptachlor } & .00 & 2,4-D & .00 \\ \text { Heptachlor epoxide } & .00 & 2,4,5-T & \end{array}$

ANALYSIS OF SELECTED GROSS RADIOACTIVITY

(Total activity concentrations in picocuries per liter)

Gross Alpha, as uranium natural 17

Gross Beta, as

cesium-137
Gross Beta, as

strontium-90 
PUBLIC WATER SUPPLY: Graceville

COUNTY: Jackson

COLLECTION DATE: $01-04-78$

SAMPLING POINT (1) Treated water -305713085305390 , tap on east side of U.S. Post office building.

(2) Raw water-

ANALYSIS OF MAJOR CHEMICAL CONSTITUENTS AND PHYSICAL PROPERTIES

(Dissolved concentrations in milligrams per liter, except as indicated)

(1)

7.3

42

Silica (SiO2)

Calcium ( $\mathrm{Ca}$ )

Magnesium ( $\mathrm{Mg}$ )

Sodium ( $\mathrm{Na}$ )

Potassium (K)

Bicarbonate ( $\mathrm{HCO} 3) \quad 110$

Carbonate ( $\mathrm{CO} 3$ )

Sulfate (S04)

Chloride (C1)

Fluoride (F)

Nitrate (NO3-N), total

Nitrite ( N02-N), total
(2)

Dissolved solids

(residue at $180^{\circ} \mathrm{C}$ )

Hardness as $\mathrm{CaCO} 3$

$$
(\mathrm{Ca}, \mathrm{Mg})
$$

(1)
.2

0

1.2

4.5

.0

$1 \cdot 3$

.00
Noncarbonate hardness as $\mathrm{CaCO} 3$

Percent sodium

Alkalinity as $\mathrm{CaCO} 3$

Specific conductance

(umhos $/ \mathrm{cm}$ at $25^{\circ} \mathrm{C}$ )

$\mathrm{pH}$ (units)

Temperature $\left({ }^{\circ} \mathrm{C}\right)$

Color (Pt-Co units)

Turbidity (NTU)
124

110

18

4

90

170

7.7

17.0

2

0

ANALYSIS OF SELECTED TRACE ELEMENTS

(Total concentrations in micrograms per liter, except as indicated)

$\begin{array}{lr}\text { Arsenic ( } \mathrm{As} \text { ) } & 2 \\ \text { Barium ( } \mathrm{Ba}) & - \\ \text { Cadmium ( } \mathrm{Cd}) & 0 \\ \text { Chromium ( } \mathrm{Cr} \text { ) } & 10 \\ \text { Copper (Cu) } & 28 \\ \text { Iron (Fe) } & 0 \\ \text { Lead ( } \mathrm{Pb} \text { ) } & 28\end{array}$

$\begin{array}{lc}\text { Manganese (Mn) } & 0 \\ \text { Mercury ( } \mathrm{Hg}) & <.5 \\ \text { Selenium ( } \mathrm{Se}) & 0 \\ \text { Silver ( } \mathrm{Ag}) & 0 \\ \text { Strontium, dissolved (Sr) } & 50 \\ \text { Zinc ( } \mathrm{Zn} \text { ) } & 100\end{array}$

ANALYSIS OF SELECTED PESTICIDE AND INDUSTRIAL COMPOUNDS

(Total concentrations in micrograms per liter)

$\begin{array}{llll}\text { Aldrin } & .00 & \text { Lindane } & .00 \\ \text { Chlordane } & .0 & \text { Methoxych lor } & .00 \\ \text { DDD } & .00 & \text { Mirex } & .00 \\ \text { DDE } & .00 & \text { PCB } & .0 \\ \text { DDT } & .00 & \text { PCN } & .00 \\ \text { Dieldrin } & .00 & \text { Silvex } & .00 \\ \text { Endrin } & .00 & \text { Toxaphene } & 0 \\ \text { Heptachlor } & .00 & 2,4-D & .00 \\ \text { Heptachlor epoxide } & .00 & 2,4,5-T & .00\end{array}$

ANALYSIS OF SELECTED GROSS RADIOACTIVITY

(Total activity concentrations in picocuries per liter)

Gross Alpha, as

uranium natural $<1.1$

Gross Beta, as

cesium-137
Gross Beta, as

strontium-90
.00

.00

0

.00

.00

.00 
PUBLIC WATER SUPPLY: Greenville

COUNTY: Madison

COLLECTION DATE: 12-08-77

SAMPLING POINT (1) Treated water-302906083380390 tap in yard on north side of City Hall/fire station.

(2) Raw water-

ANALYSIS OF MAJOR CHEMICAL CONSTITUENTS AND PHYSICAL PROPERTIES

(Dissolved concentrations in milligrams per liter, except as indicated)

$\begin{array}{lc} & (1) \\ \text { Silica (SiO2) } & 8.4 \\ \text { Calcium (Ca) } & 62 \\ \text { Magnesium (Mg) } & 9.4 \\ \text { Sodium (Na) } & 3.6 \\ \text { Potassium (K) } & .6 \\ \text { Bicarbonate (HCO3) } & 220 \\ \text { Carbonate (CO3) } & 0 \\ \text { Sulfate (SO4) } & 16 \\ \text { Chloride (C1) } & 7.6 \\ \text { Fluoride (F) } & .1 \\ \text { Nitrate (NO3-N), } & .07 \\ \quad \text { total (NO2-N), } \\ \text { Nitrite } \quad \\ \quad \text { total }\end{array}$

(2)
(1)

Dissolved solids

(residue at $180^{\circ} \mathrm{C}$ )

232

Hardness as $\mathrm{CaCO} 3$

$(\mathrm{Ca}, \mathrm{Mg})$

190

Noncarbonate hardness as $\mathrm{CaCO} 3$

Percent sodium

Alkalinity as $\mathrm{CaCO} 3$

Specific conductance

(umhos $/ \mathrm{cm}$ at $25^{\circ} \mathrm{C}$ )

$\mathrm{pH}$ (units)

Temperature $\left({ }^{\circ} \mathrm{C}\right)$

Color (Pt-Co units)

Turbidity (NTU)

ANALYSIS OF SELECTED TRACE ELEMENTS

( Total concentrations in micrograms per liter, except as indicated)

$\begin{array}{lrlc}\text { Arsenic (As) } & 1 & \text { Manganese (Mn) } & 20 \\ \text { Barium (Ba) } & 0 & \text { Mercury (Hg) } & <\cdot 5 \\ \text { Cadmium ( } \mathrm{Cd}) & 1 & \text { Selenium ( Se) } & 0 \\ \text { Chromium ( } \mathrm{Cr}) & <10 & \text { Silver (Ag) } & 0 \\ \text { Copper (Cu) } & 3 & \text { Strontium, dissolved ( Sr) } & 50 \\ \text { Iron (Fe) } & 260 & \text { Zinc (Zn) } & 40 \\ \text { Lead ( } \mathrm{Pb}) & 41 & & \end{array}$

ANALYSIS OF SELECTED PESTICIDE AND INDUSTRIAL COMPOUNDS (Total concentrations in micrograms per 1iter)

$\begin{array}{llll}\text { Aldrin } & .00 & \text { Lindane } & .00 \\ \text { Chlordane } & .0 & \text { Methoxychlor } & .00 \\ \text { DDD } & .00 & \text { Mirex } & .00 \\ \text { DDE } & .00 & \text { PCB } & .0 \\ \text { DDT } & .00 & \text { PCN } & .00 \\ \text { Dieldrin } & .00 & \text { Silvex } & .00 \\ \text { Endrin } & .00 & \text { Toxaphene } & 0 \\ \text { Heptachlor } & .00 & 2,4-D & .00 \\ \text { Heptachlor epoxide } & .00 & 2,4,5-T & .00\end{array}$

ANALYSIS OF SELECTED GROSS RADIOACTIVITY

(Total activity concentrations in picocuries per liter)

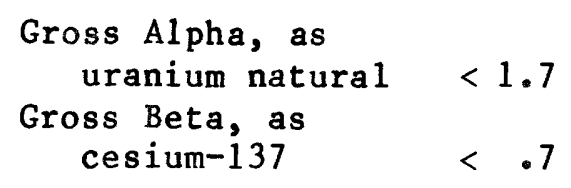

Gross Beta, as

strontium $-90<.6$ 
PUBLIC WATER SUPPLY: Groveland

COUNTY: Lake

COLLECTION DATE: 11-03-77

SAMPLING POINT (1) Treated water-283307081512701, tap outside small building near water treatment plant.

(2) Raw water-

ANALYSIS OF MAJOR CHEMICAL CONSTITUENTS AND PHYSICAL PROPERTIES

(Dissolved concentrations in milligrams per liter, except as indicated)

$\begin{array}{lc}\text { Silica (SiO2) } & 11 \\ \text { Calcium (Ca) } & 43 \\ \text { Magnesium (Mg) } & 4.4 \\ \text { Sodium (Na) } & 3.7 \\ \text { Potassium (K) } & .7 \\ \text { Bicarbonate (HCO3) } & 140 \\ \text { Carbonate (CO3) } & 0 \\ \text { Sulfate (SO4) } & 1.9 \\ \text { Chloride (C1) } & 8.5 \\ \text { Fluoride (F) } & .1 \\ \text { Nitrate (NO3-N), } & .15 \\ \quad \text { total (NO2-N), } \\ \text { Nitrite (NOtal }\end{array}$

(2)

(1)

Dissolved solids

$$
\text { (residue at } 180^{\circ} \mathrm{C} \text { ) } \quad 141
$$

Hardness as $\mathrm{CaCO} 3$

$(\mathrm{Ca}, \mathrm{Mg})$

Noncarbonate hardness

as $\mathrm{CaCO} 3$

Percent sodium

Alkalinity as $\mathrm{CaCO} 3$

Specific conductance

(umhos $/ \mathrm{cm}$ at $25^{\circ} \mathrm{C}$ )

$\mathrm{pH}$ (units)

Temperature $\left({ }^{\circ} \mathrm{C}\right)$

Color (Pt-Co units)

Turbidity (NTU)

130

11

6

110

281

7.2

24.0

0

1

ANALYSIS OF SELECTED TRACE ELEMENTS

(Total concentrations in micrograms per liter, except as indicated)

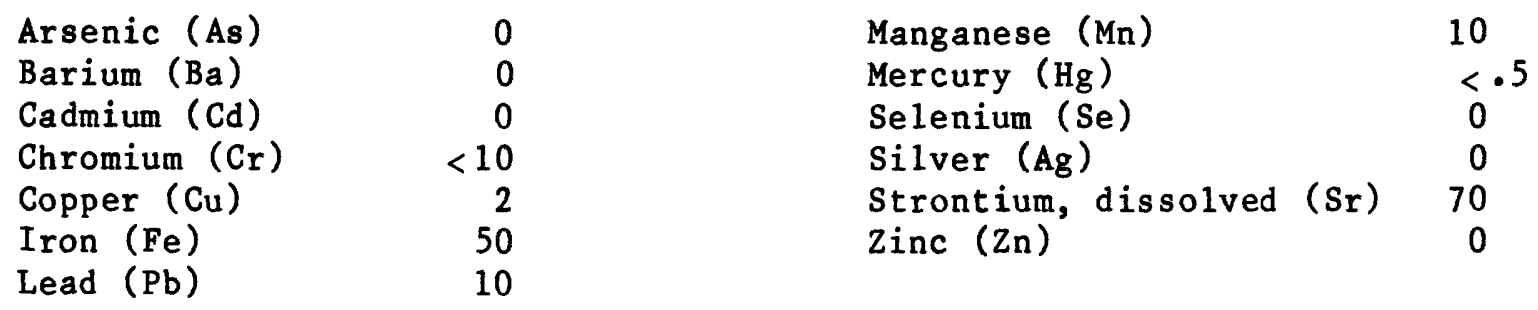

ANALYSIS OF SELECTED PESTICIDE AND INDUSTRIAL COMPOUNDS

( Total concentrations in micrograms per 1iter)

$\begin{array}{lllr}\text { Aldrin } & .00 & \text { Lindane } & .00 \\ \text { Chlordane } & .0 & \text { Methoxychlor } & .00 \\ \text { DDD } & .00 & \text { Mirex } & .0 \\ \text { DDE } & .00 & \text { PCB } & .00 \\ \text { DDT } & .00 & \text { PCN } & .00 \\ \text { Dieldrin } & .00 & \text { Silvex } & 0 \\ \text { Endrin } & .00 & \text { Toxaphene } & .00 \\ \text { Heptachlor } & .00 & 2,4-D & .00 \\ \text { Heptachlor epoxide } & .00 & 2,4,5-\mathrm{T} & \end{array}$

ANALYSIS OF SELECTED GROSS RADIOACTIVITY

(Total activity concentrations in picocuries per liter)

Gross Alpha, as uranium natural

Gross Beta, as

cesium-137
Gross Beta, as

strontium-90 
PUBLIC WATER SUPPLY: Havana

COUNTY: Gadsden

COLLECTION DATE: 11-22-77

SAMPLING POINT (1) Treated water-303739084245190, tap on east side near rear door of U.S. Post Office building.

(2) Raw water-

ANALYSIS OF MAJOR CHEMICAL CONSTITUENTS AND PHYSICAL PROPERTIES

(Dissolved concentrations in milligrams per liter, except as indicated)

(1) (2)

$\begin{array}{lc}\text { Silica (SiO2) } & 26 \\ \text { Calcium (Ca) } & 81 \\ \text { Magnesium (Mg) } & 27 \\ \text { Sodium (Na) } & 22 \\ \text { Potassium (K) } & 2.0 \\ \text { Bicarbonate (HCO3) } & 150 \\ \text { Carbonate (CO3) } & 0 \\ \text { Sulfate (SO4) } & 180 \\ \text { Chloride (C1) } & 31 \\ \text { Fluoride (F) } & .1 \\ \text { Nitrate (NO3-N), } & \\ \quad \text { total (NO2-N), } & .03 \\ \text { Nitrite (NO2-N), } & \\ \quad \text { total } & .00\end{array}$

Dissolved solids

(1)

(residue at $180^{\circ} \mathrm{C}$ ) $\quad 460$

Hardness as $\mathrm{CaCO} 3$

$(\mathrm{Ca}, \mathrm{Mg})$

310

Noncarbonate hardness as $\mathrm{CaCO} 3$

190

Percent sodium 13

Alkalinity as $\mathrm{CaCO} 3 \quad 120$

Specific conductance

(umhos $/ \mathrm{cm}$ at $25^{\circ} \mathrm{C}$ )

$\mathrm{pH}$ (units)

Temperature $\left({ }^{\circ} \mathrm{C}\right)$

Color (Pt-Co units)

Turbidity (NTU)
690

7.8

22.5

0

1

ANALYSIS OF SELECTED TRACE ELEMENTS

(Total concentrations in micrograms per liter, except as indicated)

$\begin{array}{lr}\text { Arsenic (As) } & 4 \\ \text { Barium (Ba) } & 0 \\ \text { Cadmium (Cd) } & 3 \\ \text { Chromium (Cr) } & 10 \\ \text { Copper (Cu) } & 57 \\ \text { Iron (Fe) } & 90 \\ \text { Lead (Pb) } & 23\end{array}$

$\begin{array}{lc}\text { Manganese (Mn) } & 0 \\ \text { Mercury (Hg) } & <.5 \\ \text { Selenium ( Se) } & 0 \\ \text { Silver (Ag) } & 0 \\ \text { Strontium, dissolved ( } \mathrm{Sr}) & 880 \\ \text { Zinc ( } \mathrm{Zn} \text { ) } & 10\end{array}$

ANALYSIS OF SELECTED PESTICIDE AND INDUSTRIAL COMPOUNDS

(Total concentrations in micrograms per liter)

$\begin{array}{llll}\text { Aldrin } & .00 & \text { Lindane } & .00 \\ \text { Chlordane } & .0 & \text { Methoxychlor } & .00 \\ \text { DDD } & .00 & \text { Mirex } & .00 \\ \text { DDE } & .00 & \text { PCB } & .0 \\ \text { DDT } & .00 & \text { PCN } & .00 \\ \text { Dieldrin } & .00 & \text { Silvex } & .00 \\ \text { Endrin } & .00 & \text { Toxaphene } & 0 \\ \text { Heptachlor } & .00 & 2,4-D & .00 \\ \text { Heptachlor epoxide } & .00 & 2,4,5-T & .00\end{array}$

ANALYSIS OF SELECTED GROSS RADIOACTIVITY

(Total activity concentrations in picocuries per liter)

Gross Alpha, as uranium natural

Gross Beta, as

cesium-137
Gross Beta, as

strontium -90 
PUBLIC WATER SUPPLY: Hilliard

COUNTY: Nassau

COLLECTION DATE: 12-14-77

SAMPLING POINT (1) Treated water-304122081550801, tap on front of building 110 at Pecan Street water treatment plant.

(2) Raw water-

ANALYSIS OF MAJOR CHEMICAL CONSTITUENTS AND PHYSICAL PROPERTIES (Dissolved concentrations in milligrams per liter, except as indicated)

$\begin{array}{lc}\text { Silica (SiO2) } & 44 \\ \text { Calcium (Ca) } & 65 \\ \text { Magnesium (Mg) } & 32 \\ \text { Sodium (Na) } & 18 \\ \text { Potassium (K) } & 2.2 \\ \text { Bicarbonate (HCO3) } & 190 \\ \text { Carbonate (CO3) } & 0 \\ \text { Sulfate (SO4) } & 120 \\ \text { Chloride (C1) } & 32 \\ \text { Fluoride (F) } & .5 \\ \text { Nitrate (NO3-N), } & \\ \quad \text { total (NO2-N), } & .04 \\ \text { Nitrite (NO2-N), } \\ \quad \text { total }\end{array}$

(1) (2)
Dissolved solids

(residue at $180^{\circ} \mathrm{C}$ ) $\quad 411$

Hardness as $\mathrm{CaCO} 3$

( Ca, Mg) 290

Noncarbonate hardness as $\mathrm{CaCO} 3$

140

Percent sodium 12

Alkalinity as $\mathrm{CaCO} 3 \quad 160$

Specific conductance

(umhos $/ \mathrm{cm}$ at $25^{\circ} \mathrm{C}$ )

$\mathrm{pH}$ (units)

655

Temperature $\left({ }^{\circ} \mathrm{C}\right)$

Color (Pt-Co units)

Turbidity (NTU)
(1)

ANALYSIS OF SELECTED TRACE ELEMENTS

(Total concentrations in micrograms per liter, except as indicated)

$\begin{array}{lrlr}\text { Arsenic (As) } & 1 & \text { Manganese (Mn) } & 0 \\ \text { Barium (Ba) } & -- & \text { Mercury (Hg) } & <.5 \\ \text { Cadmium ( Cd) } & 0 & \text { Selenium ( Se) } & 0 \\ \text { Chromium (Cr) } & 30 & \text { Silver (Ag) } & 1 \\ \text { Copper (Cu) } & 14 & \text { Strontium, dissolved (Sr) } & 510 \\ \text { Iron (Fe) } & 50 & \text { Zinc (Zn) } & 30 \\ \text { Lead (Pb) } & 6 & & \end{array}$

ANALYSIS OF SELECTED PESTICIDE AND INDUSTRIAL COMPOUNDS

(Total concentrations in micrograms per liter)

$\begin{array}{llll}\text { Aldrin } & .00 & \text { Lindane } & .00 \\ \text { Chlordane } & .0 & \text { Methoxychlor } & .00 \\ \text { DDD } & .00 & \text { Mirex } & .00 \\ \text { DDE } & .00 & \text { PCB } & .0 \\ \text { DDT } & .00 & \text { PCN } & .00 \\ \text { Dieldrin } & .00 & \text { Silvex } & .00 \\ \text { Endrin } & .00 & \text { Toxaphene } & 0 \\ \text { Heptachlor } & .00 & 2,4-D & .00 \\ \text { Heptachlor epoxide } & .00 & 2,4,5-T & .00\end{array}$

ANALYSIS OF SELECTED GROSS RADIOACTIVITY

(Total activity concentrations in picocuries per liter)

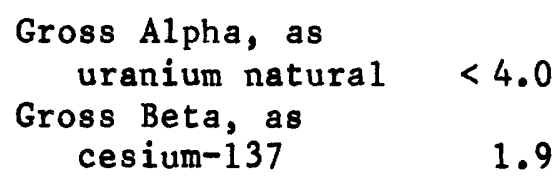

Gross Beta, as

strontium-90 
PUBLIC WATER SUPPLY: Hobe Sound

COUNTY: Martin

COLLECTION DATE: $11-30-77$

SAMPLING POINT (1) Treated water-270306080073002, tap on South Jupiter Is land near " $S$ " curve in state Road 707.

(2) Raw water-

ANALYSIS OF MAJOR CHEMICAL CONSTITUENTS AND PHYSICAL PROPERTIES (Dissolved concentrations in milligrams per liter, except as indicated)

(1) (2)

Silica (SiO2)
Calcium ( $\mathrm{Ca})$
Magnesium (Mg)
Sodium (Na)
Potassium (K)
Bicarbonate (HCO3)
Carbonate (CO3)
Sulfate (SO4)
Chloride (C1)
Fluoride (F)
Nitrate (NO3-N),
$\quad$ tota1
Nitrite (NO2-N),
$\quad$ rotal

(1)

\section{ANALYSIS OF SELECTED TRACE ELEMENTS}

(Total concentrations in micrograms per liter, except as indicated)

$\begin{array}{lr}\text { Arsenic (As) } & 0 \\ \text { Barium (Ba) } & - \\ \text { Cadmium (Cd) } & 2 \\ \text { Chromium (Cr) } & 10 \\ \text { Copper (Cu) } & 11 \\ \text { Iron (Fe) } & 190 \\ \text { Lead ( } \mathrm{Pb}) & 39\end{array}$

Manganese (Mn)

50

Mercury ( $\mathrm{Hg}$ )

Selenium ( $\mathrm{Se}$ )

Silver $(\mathrm{Ag})$

Strontium, dissolved ( $\mathrm{Sr}$ ) 160 Zinc $(\mathrm{Zn})$

ANALYSIS OF SELECTED PESTICIDE AND INDUSTRIAL COMPOUNDS

(Total concentrations in micrograms per liter)

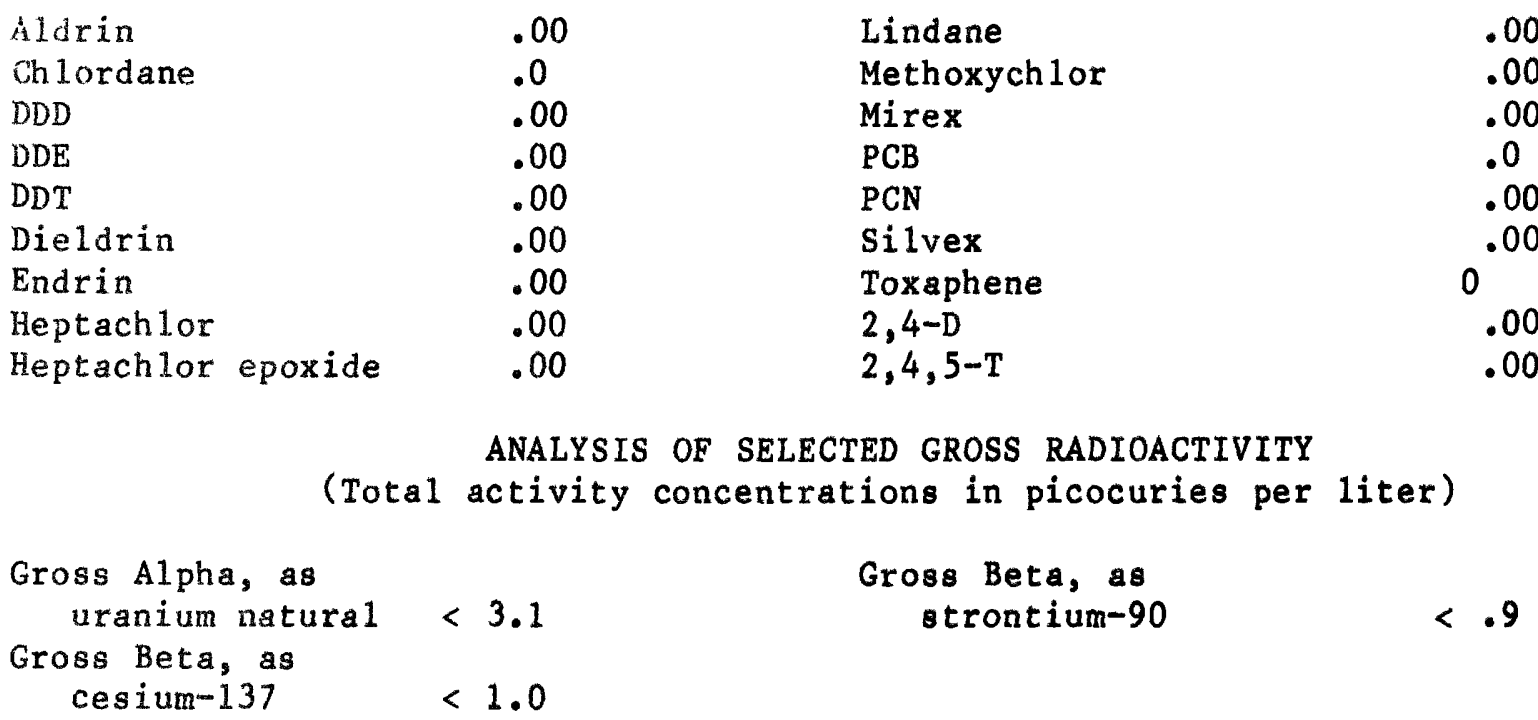


PUBLIC WATER SUPPLY: Holiday Lake Estates

COUNTY: Pasco

COLLECT LON DATE: $\quad 12-21-77$

SAMPLING POINT (1) Treated water-281023082453109, tap on hulding tank

near pumphouse.

(2) Raw water-

ANALYSIS OF MAJOR CHEMICAL CONSTITUENTS AND PHYSICAL PROPERTIES (Dissolved concentrations in milligrams per liter, except as indicated)

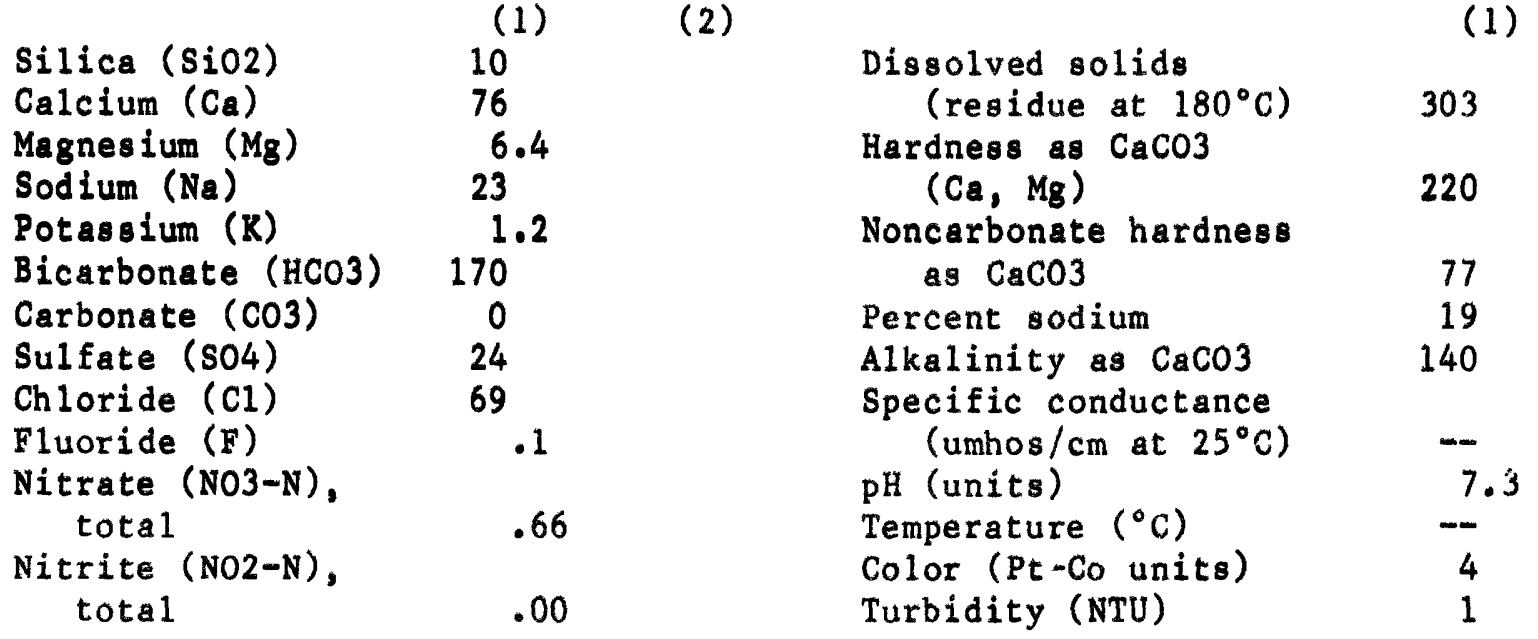

ANALYSIS OF SELECTED TRACE ELEMENTS

(Total concentrations in micrograms per liter, except as indicated)

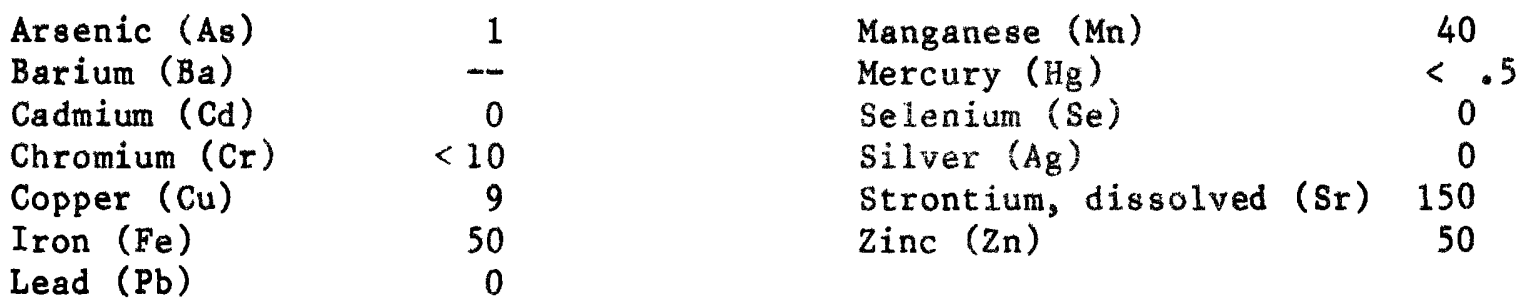

ANALYSIS OF SELECTED PESTICIDE AND INDUSTRIAL COMPOUNDS

(Total concentrations in micrograms per 1iter)

$\begin{array}{llll}\text { Aldrin } & .00 & \text { Lindane } & .00 \\ \text { Chlordane } & .0 & \text { Methoxychlor } & .00 \\ \text { DDD } & .00 & \text { Mirex } & .00 \\ \text { DDE } & .00 & \text { PCB } & .0 \\ \text { DDT } & .00 & \text { PCN } & .00 \\ \text { Dieldrin } & .00 & \text { Silvex } & .00 \\ \text { Endrin } & .00 & \text { Toxaphene } & 0 \\ \text { Heptachlor } & .00 & 2,4-D & .00 \\ \text { Heptachlor epoxide } & .00 & 2,4,5-T & .00\end{array}$

ANALYSIS OF SELECTED GROSS RADIOACTIVITY

(Total activity concentrations in picocuries per liter)

Gross Alpha, as

uranium natural $<1.7$

Gross Beta, as

cesium-137
Gross Beta, as

strontium -90

3.1 
PUBLIC WATER SUPPLY: Holly Hill

COUNTY: Volusia

COLLECTION DATE: 11-02-77

SAMPLING POINT (1) Treated water-291444081022201, tap at water treatment plant.

(2) Raw water-

ANALYSIS OF MAJOR CHEMICAL CONSTITUENTS AND PHYSICAL PROPERTIES

(Dissolved concentrations in milligrams per liter, except as indicated)

$\begin{array}{lc}\text { Silica (SiO2) } & 6.9 \\ \text { Calcium (Ca) } & 49 \\ \text { Magnesium (Mg) } & 11 \\ \text { Sodium (Na) } & 85 \\ \text { Potassium (K) } & 2.6 \\ \text { Bicarbonate (HCO3) } & 120 \\ \text { Carbonate (CO3) } & 0 \\ \text { Sulfate (SO4) } & 20 \\ \text { Chloride (C1) } & 160 \\ \text { Fluoride (F) } & .1 \\ \text { Nitrate (NO3-N), } & \\ \quad \text { total (NO2-N), } & .04 \\ \text { Nitrite } \quad & \\ \quad \text { total } & .00\end{array}$

(2)

(1)

(2)

Dissolved solids

(residue at $180^{\circ} \mathrm{C}$ ) $\quad 400$

Hardness as $\mathrm{CaCO} 3$

$(\mathrm{Ca}, \mathrm{Mg}$ )

170

Noncarbonate hardness

as $\mathrm{CaCO} 3$

70

Percent sodium 52

Alkalinity as $\mathrm{CaCO} 3 \quad 98$

Specific conductance

(umhos $/ \mathrm{cm}$ at $25^{\circ} \mathrm{C}$ )

$\mathrm{pH}$ (units)

Temperature $\left({ }^{\circ} \mathrm{C}\right)$

Color (Pt-Co units)

Turbidity (NTU)

8.6

23.0

12

ANALYSIS OF SELECTED TRACE ELEMENTS

( Total concentrations in micrograms per liter, except as indicated)

$\begin{array}{lrlc}\text { Arsenic (As) } & 0 & \text { Manganese (Mn) } & 0 \\ \text { Barium (Ba) } & 0 & \text { Mercury (Hg) } & <.5 \\ \text { Cadmium (Cd) } & 0 & \text { Selenium (Se) } & 0 \\ \text { Chromium (Cr) } & 10 & \text { Silver (Ag) } & 0 \\ \text { Copper ( Cu) } & 5 & \text { Strontium, dissolved (Sr) } & 920 \\ \text { Iron (Fe) } & 130 & \text { Zinc (Zn) } & 830 \\ \text { Lead (Pb) } & 9 & & \end{array}$

ANALYSIS OF SELECTED PESTICIDE AND INDUSTRIAL COMPOUNDS (Total concentrations in micrograms per 1iter)

$\begin{array}{llll}\text { Aldrin } & .00 & \text { Lindane } & .00 \\ \text { Chlordane } & .0 & \text { Methoxych1or } & .00 \\ \text { DDD } & .00 & \text { Mirex } & .00 \\ \text { DDE } & .00 & \text { PCB } & .0 \\ \text { DDT } & .00 & \text { PCN } & .00 \\ \text { Dieldrin } & .00 & \text { Silvex } & .00 \\ \text { Endrin } & .00 & \text { Toxaphene } & 0 \\ \text { Heptachlor } & .00 & 2,4-D & .00 \\ \text { Heptachlor epoxide } & .00 & 2,4,5-\mathrm{T} & .00\end{array}$

ANALYSIS OF SELECTED GROSS RADIOACTIVITY

(Total activity concentrations in picocuries per liter)

Gross Alpha, as

uranium natural -

Gross Beta, as

cesium-137
Gross Beta, as

strontium -90 
PUBLIC WATER SUPPLY: Hudson Community Water Works

COUNTY: Pasco

COLLECTION DATE: 12-19-77

SAMPLING POINT (1) Treated water-282144082414709, tap at elevated water tank.

(2) Raw water-

ANALYSIS OF MAJOR CHEMICAL CONSTITUENTS AND PHYSICAL PROPERTIES (Dissolved concentrations in milligrams per liter, except as indicated)

(1) (2)

$\begin{array}{lc}\text { Silica (SiO2) } & 10 \\ \text { Calcium (Ca) } & 61 \\ \text { Magnesium (Mg) } & 5.3 \\ \text { Sodium (Na) } & 41 \\ \text { Potassium (K) } & 1.7 \\ \text { Bicarbonate (HCO3) } & 160 \\ \text { Carbonate (CO3) } & 0 \\ \text { Sulfate (SO4) } & 14 \\ \text { Chloride (C1) } & 81 \\ \text { Fluoride (F) } & .1 \\ \text { Nitrate (NO3-N), } & \\ \quad \text { total (NO2-N), } & .27 \\ \text { Nitrite (NOtal } & \\ \quad \text { total }\end{array}$

(1)

\section{ANALYSIS OF SELECTED TRACE ELEMENTS}

( Total concentrations in micrograms per liter, except as indicated)

$\begin{array}{lrlr}\text { Arsenic (As) } & 1 & \text { Manganese (Mn) } & 0 \\ \text { Barium (Ba) } & - & \text { Mercury (Hg) } & <\cdot 5 \\ \text { Cadmium ( } \mathrm{Cd}) & 0 & \text { Selenium (Se) } & 0 \\ \text { Chromium (Cr) } & 10 & \text { Silver (Ag) } & 0 \\ \text { Copper (Cu) } & 7 & \text { Strontium, dissolved (Sr) } & 230 \\ \text { Iron (Fe) } & 10 & \text { Zinc (Zn) } & 20 \\ \text { Lead (Pb) } & 12 & & \end{array}$

ANALYSIS OF SELECTED PESTICIDE AND INDUSTRIAL COMPOUNDS

(Total concentrations in micrograms per liter)

$\begin{array}{llll}\text { Aldrin } & .00 & \text { Lindane } & .00 \\ \text { Chlordane } & .0 & \text { Methoxychlor } & .00 \\ \text { DDD } & .00 & \text { Mirex } & .00 \\ \text { DDE } & .00 & \text { PCB } & .0 \\ \text { DDT } & .00 & \text { PCN } & .00 \\ \text { Dieldrin } & .00 & \text { Silvex } & .00 \\ \text { Endrin } & .00 & \text { Toxaphene } & 0 \\ \text { Heptachlor } & .00 & 2,4-D & .00 \\ \text { Heptachlor epoxide } & .00 & 2,4,5-\mathrm{T} & .00\end{array}$

ANALYSIS OF SELECTED GROSS RADIOACTIVITY

(Total activity concentrations in picocuries per liter)

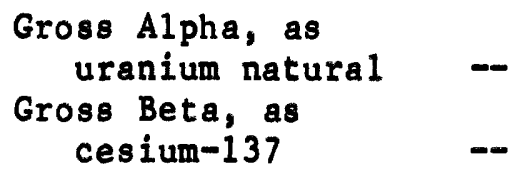

Gross Beta, as

strontium -90 
PUBLIC WATER SUPPLY: Immokalee

COUNTY: Collier

COLLECTION DATE: 11-29-77

SAMPLING POINT (1) Treated water-262436081254101, tap at Farmers Village,

2 miles south of Immokalee Airport on Florida route 29.

(2) Raw water-

ANALYSIS OF MAJOR CHEMICAL CONSTITUENTS AND PHYSICAL PROPERTIES

(Dissolved concentrations in milligrams per liter, except as indicated)
(1)
(2)
(1)

\begin{tabular}{|c|c|}
\hline \multirow{2}{*}{\multicolumn{2}{|c|}{$\begin{array}{ll}\text { Silica (SiO2) } & 31 \\
\text { Calcium (Ca) } & 73\end{array}$}} \\
\hline & \\
\hline \\
\hline \multicolumn{2}{|c|}{$\begin{array}{lc}\text { Calcium (Ca) } & 73 \\
\text { Magnesium (Mg) } & 17 \\
\text { Sodium (Na) } & 18 \\
\text { Potassium (R) } & 2.4\end{array}$} \\
\hline \multirow{2}{*}{\multicolumn{2}{|c|}{$\begin{array}{l}\text { Carbonate (CO3) } \\
\text { Sulfate (s04) }\end{array}$}} \\
\hline Sulfate ( $\mathrm{S04})$ & \\
\hline hloride (Cl) & 24 \\
\hline Fluoride (F) & .3 \\
\hline \multirow{2}{*}{$\begin{array}{l}\text { Nitrate }(\mathrm{NO3}-\mathrm{N}), \\
\text { total } \\
\text { Nitrite }(\text { NO2-N), } \\
\text { total }\end{array}$} & \\
\hline & \\
\hline
\end{tabular}

Dissolved solids (residue at $180^{\circ} \mathrm{C}$ ) 326

Hardness as $\mathrm{CaCO} 3$

$(\mathrm{Ca}, \mathrm{Mg}) \quad 250$

Noncarbonate hardness as $\mathrm{CaCO} 3$

Percent sodium

Alkalinity as $\mathrm{CaCO} 3$

Specific conductance

(umhos $/ \mathrm{cm}$ at $25^{\circ} \mathrm{C}$ )

$\mathrm{pH}$ (units)

Temperature $\left({ }^{\circ} \mathrm{C}\right)$

Color (Pt-Co units)

Turbidity (NTU)

ANALYSIS OF SELECTED TRACE ELEMENTS

(Total concentrations in micrograms per liter, except as indicated)

$\begin{array}{lrlc}\text { Arsenic (As) } & 0 & \text { Manganese (Mn) } & 0 \\ \text { Barium (Ba) } & - & \text { Mercury (Hg) } & <.5 \\ \text { Cadmium (Cd) } & 0 & \text { Selenium (Se) } & 0 \\ \text { Chromium (Cr) } & 20 & \text { Silver (Ag) } & 0 \\ \text { Copper (Cu) } & 8 & \text { Strontium, dissolved (Sr) } & 190 \\ \text { Iron (Fe) } & 150 & \text { Zinc ( } \mathrm{n} \text { ) } & 10 \\ \text { Lead (Pb) } & 17 & & \end{array}$

ANALYSIS OF SELECTED PESTICIDE AND INDUSTRIAL COMPOUNDS

(Total concentrations in micrograms per liter)

$\begin{array}{llll}\text { Aldrin } & .00 & \text { Lindane } & .00 \\ \text { Chlordane } & .0 & \text { Methoxychlor } & .00 \\ \text { DDD } & .00 & \text { Mirex } & .00 \\ \text { DDE } & .00 & \text { PCB } & .0 \\ \text { DDT } & .00 & \text { PCN } & .00 \\ \text { Dieldrin } & .00 & \text { Silvex } & .00 \\ \text { Endrin } & .00 & \text { Toxaphene } & 0 \\ \text { Heptachlor } & .00 & 2,4-D & .00 \\ \text { Heptachlor epoxide } & .00 & 2,4,5-T & .00\end{array}$

ANALYSIS OF SELECTED GROSS RADIOACTIVITY

(Total activity concentrations in picocuries per liter)

Gross Alpha, as

uranium natural --

Gross Beta, as

cesium-137
Gross Beta, as

strontium -90 
PUBLIC WATER SUPPLY: Imperial Lakes

COUNTY: POlK

COLLECTION DATE: 12-15-77

SAMPLING POINT (1) Treated water-275605081583909, tap on holding tank near pumphouse.

(2) Raw water-

ANALYSIS OF MAJOR CHEMICAL CONSTITUENTS AND PHYSICAL PROPERTIES (Dissolved concentrations in milligrams per liter, except as indicated)

(1) (2)

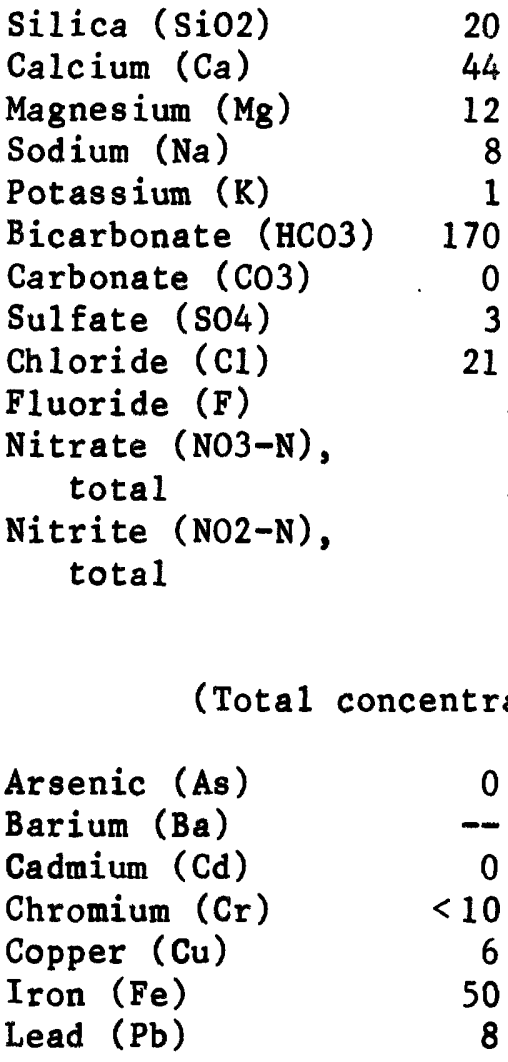

(1)

20

44

12

8.2

1.0

170

0

3.7

21

.3

.02

.00
Dissolved solids

(residue at $180^{\circ} \mathrm{C}$ ) 206

Hardness as $\mathrm{CaCO} 3$

$(\mathrm{Ca}, \mathrm{Mg})$

160

Noncarbonate hardness as $\mathrm{CaCO} 3$

20

Percent sodium

Alkalinity as $\mathrm{CaCO} 3$

Specific conductance

(umhos $/ \mathrm{cm}$ at $25^{\circ} \mathrm{C}$ )

pH (units)

Temperature $\left({ }^{\circ} \mathrm{C}\right)$

Color (Pt-Co units)

Turbidity (NTU)
10

140

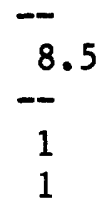

ANALYSIS OF SELECTED TRACE ELEMENTS

ANALYSIS OF SELECTED PESTICIDE AND INDUSTRIAL COMPOUNDS (Total concentrations in micrograms per liter)

$\begin{array}{llll}\text { Aldrin } & .00 & \text { Lindane } & .00 \\ \text { Chlordane } & .0 & \text { Methoxychlor } & .00 \\ \text { DDD } & .00 & \text { Mirex } & .00 \\ \text { DDE } & .00 & \text { PCB } & .0 \\ \text { DDT } & .00 & \text { PCN } & .00 \\ \text { Dieldrin } & .00 & \text { Silvex } & .00 \\ \text { Endrin } & .00 & \text { Toxaphene } & 0 \\ \text { Heptachlor } & .00 & 2,4-D & .00 \\ \text { Heptachlor epoxide } & .00 & 2,4,5-\mathrm{T} & .00\end{array}$

ANALYSIS OF SELECTED GROSS RADIOACTIVITY

(Total activity concentrations in picocuries per liter)

Gross Alpha, as uranium natural $<2.0$

Gross Beta, as

cesium-137
Gross Beta, as

strontium-90

$\begin{array}{lc}\text { Manganese (Mn) } & 0 \\ \text { Mercury ( } \mathrm{Hg}) & <.5 \\ \text { Selenium ( } \mathrm{Se}) & 0 \\ \text { Silver (Ag) } & 0 \\ \text { Strontium, dissolved ( } \mathrm{Sr}) & 290 \\ \text { Zinc ( } \mathrm{Zn}) & 10\end{array}$

Manganese (Mn)

$<.5$

0

10 
PUBLIC WATER SUPPLY: Indian Hills

COUNTY: Seminole COLLECTION DATE: 11-14-77

SAMPLING POINT (1) Treated water-283823081195001, tap outside (south side) on storage tank near aeration tank at water treatment plant.

(2) Raw water-

ANALYSIS OF MAJOR CHEMICAL CONSTITUENTS AND PHYSICAL PROPERTIES (Dissolved concentrations in milligrams per liter, except as indicated)

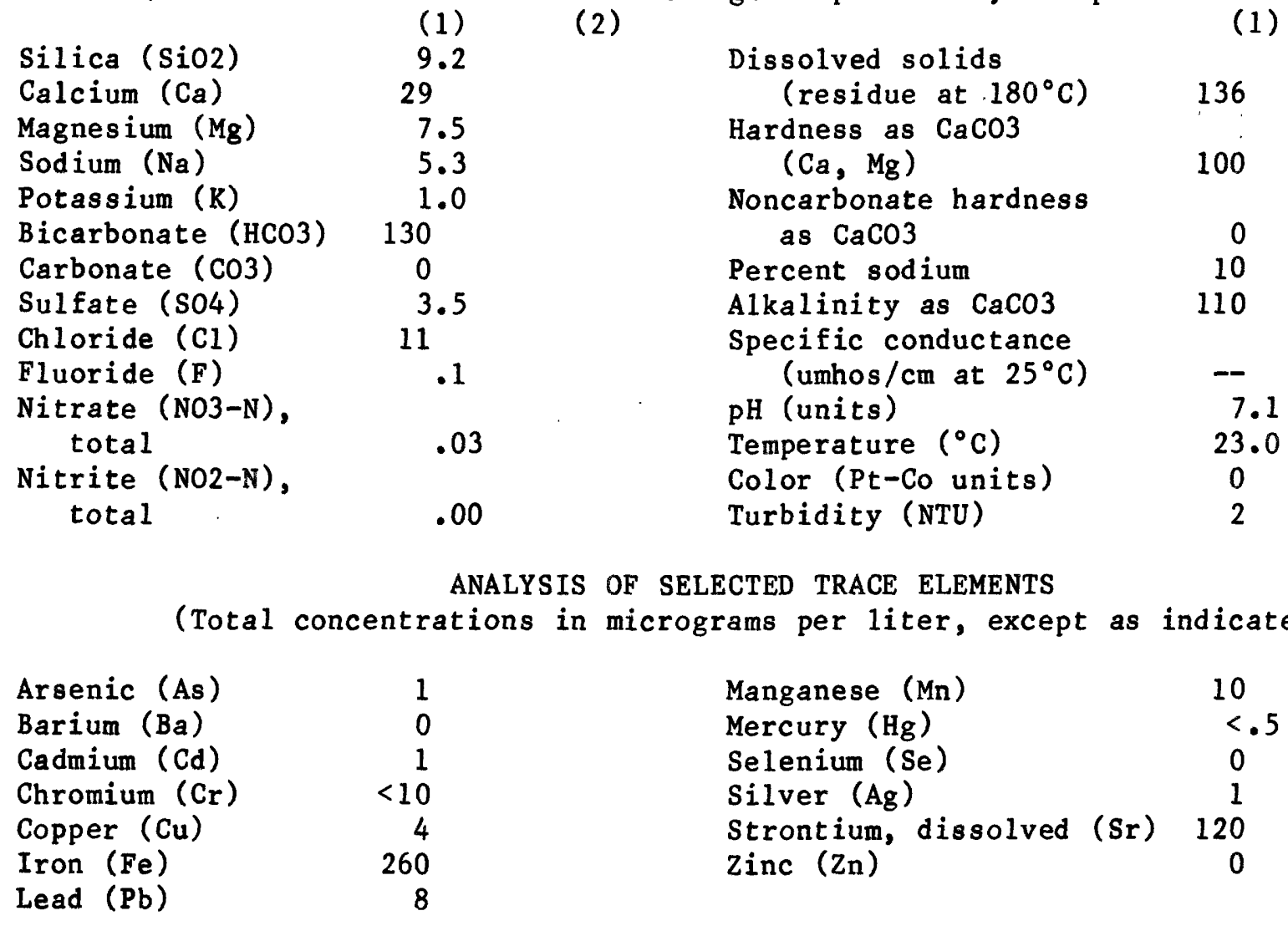

ANALYSIS OF SELECTED PESTICIDE AND INDUSTRIAL COMPOUNDS (Total concentrations in micrograms per liter)

$\begin{array}{llll}\text { Aldrin } & .00 & \text { Lindane } & .00 \\ \text { Chlordane } & .0 & \text { Methoxychlor } & .00 \\ \text { DDD } & .00 & \text { Mirex } & .00 \\ \text { DDE } & .00 & \text { PCB } & .0 \\ \text { DDT } & .00 & \text { PCN } & .00 \\ \text { Dieldrin } & .00 & \text { Silvex } & .00 \\ \text { Endrin } & .00 & \text { Toxaphene } & 0 \\ \text { Heptachlor } & .00 & 2,4-D & .00 \\ \text { Heptachlor epoxide } & .00 & 2,4,5-T & .00\end{array}$

ANALYSIS OF SELECTED GROSS RADIOACTIVITY

(Total activity concentrations in picocuries per liter)

Gross Alpha, as

uranium natural $<1.4$

Gross Beta, as

cesium-137
Gross Beta, as

strontium -90

$<\cdot 8$ 
PUBLIC WATER SUPPLY: Indiantown

COUNTY: Martin

COLLECTION DATE: 12-06-77

SAMPLING POINT (1) Treated water-270109080281002, tap inside water treatment plant (West Brook addition).

(2) Raw water-

ANALYSIS OF MAJOR CHEMICAL CONSTITUENTS AND PHYSICAL PROPERTIES

(Dissolved concentrations in milligrams per liter, except as indicated)

$\begin{array}{lc}\text { Silica (SiO2) } & 17 \\ \text { Calcium (Ca) } & 110 \\ \text { Magnesium (Mg) } & 3,0 \\ \text { Sodium (Na) } & 14 \\ \text { Potassium (K) } & 1.8 \\ \text { Bicarbonate (HCO3) } & 284 \\ \text { Carbonate (CO3) } & 0 \\ \text { Sulfate (SO4) } & 3.0 \\ \text { Chloride (C1) } & 37 \\ \text { Fluoride (F) } & .2 \\ \text { Nitrate (NO3-N), } \\ \quad \text { total } \\ \text { Nitrite (NO2-N), } \\ \quad \text { total }\end{array}$

(2)

$\begin{array}{lc}\text { Dissolved solids } & \\ \quad \text { (residue at } 180^{\circ} \mathrm{C} \text { ) } & 376 \\ \text { Hardness as } \mathrm{CaCO} & \\ \quad \text { (Ca, Mg) } & 290 \\ \text { Noncarbonate hardness } & \\ \quad \text { as CaCO3 } & 57 \\ \text { Percent sodium } & 10 \\ \text { Alkalinity as CaCO3 } & 233 \\ \text { Specific conductance } & \\ \quad \text { (umhos/cm at } 25^{\circ} \mathrm{C} \text { ) } & 550 \\ \text { pH (units) } & 8.0 \\ \text { Temperature ( }{ }^{\circ} \mathrm{C} \text { ) } & 24.5 \\ \text { Color (Pt-Co units) } & 6 \\ \text { Turbidity (NTU) } & 1\end{array}$

ANALYSIS OF SELECTED TRACE ELEMENTS

(Total concentrations in micrograms per liter, except as indicated)

$\begin{array}{lrlr}\text { Arsenic (As) } & 0 & \text { Manganese (Mn) } & 10 \\ \text { Barium (Ba) } & 0 & \text { Mercury (Hg) } & <.5 \\ \text { Cadmium (Cd) } & 1 & \text { Selenium ( Se) } & 0 \\ \text { Chromium (Cr) } & <10 & \text { Silver (Ag) } & 0 \\ \text { Copper (Cu) } & 7 & \text { Strontium, dissolved ( Sr) } & 680 \\ \text { Iron (Fe) } & 50 & \text { Zinc (Zn) } & 40 \\ \text { Lead (Pb) } & 18 & & \end{array}$

ANALYSIS OF SELECTED PESTICIDE AND INDUSTRIAL COMPOUNDS (Total concentrations in micrograms per liter)

$\begin{array}{llll}\text { Aldrin } & .00 & \text { Lindane } & .00 \\ \text { Chlordane } & .0 & \text { Methoxychlor } & .00 \\ \text { DDD } & .00 & \text { Mirex } & .00 \\ \text { DDE } & .00 & \text { PCB } & .0 \\ \text { DDT } & .00 & \text { PCN } & .00 \\ \text { Dieldrin } & .00 & \text { Silvex } & .00 \\ \text { Endrin } & .00 & \text { Toxaphene } & 0 \\ \text { Heptachlor } & .00 & 2,4-D & .04 \\ \text { Heptachlor epoxide } & .00 & 2,4,5-\mathrm{T} & .00\end{array}$

ANALYSIS OF SELECTED GROSS RADIOACTIVITY

(Total activity concentrations in picocuries per liter)

Gross Alpha, as uranium natural

Gross Beta, as

cesium-137
Gross Beta, as

strontium-90 
PUBLIC WATER SUPPLY: Interlachen

COUNTY: Putnam

COLLECTION DATE: 11-03-77

SAMPLING POINT (1) Treated water -293720081534501 , tap at water treatment plant.

(2) Raw water-

ANALYSIS OF MAJOR CHEMICAL CONSTITUENTS AND PHYSICAL PROPERTIES (Dissolved concentrations in milligrams per liter, except as indicated)

$\begin{array}{lc}\text { Silica (SiO2) } & (1) \\ \text { Calcium (Ca) } & 9.8 \\ \text { Magnesium (Mg) } & 24 \\ \text { Sodium (Na) } & 6.3 \\ \text { Potassium (K) } & 3.4 \\ \text { Bicarbonate (HCO3) } & 100 \\ \text { Carbonate (CO3) } & 0 \\ \text { Sulfate (SO4) } & .5 \\ \text { Chloride (C1) } & 5.2 \\ \text { Fluoride (F) } & .0 \\ \text { Nitrate (NO3-N), } & .08 \\ \quad \text { total (NO2-N), } & \\ \text { Nitrite (Notal } & .00 \\ \quad \text { total }\end{array}$

(2)

Dissolved solids

(residue at $180^{\circ} \mathrm{C}$ ) $\quad 102$

Hardness as $\mathrm{CaCO} 3$

( $\mathrm{Ca}, \mathrm{Mg}$ )

Noncarbonate hardness

as $\mathrm{CaCO} 3$

Percent sodium

Alkalinity as $\mathrm{CaCO} 3$

Specific conductance

(umhos $/ \mathrm{cm}$ at $25^{\circ} \mathrm{C}$ )

$\mathrm{pH}$ (units)

Temperature $\left({ }^{\circ} \mathrm{C}\right)$

Color ( $\mathrm{Pt}-\mathrm{Co}$ units)

Turbidity (NTU)
(1)

ANALYSIS OF SELECTED TRACE ELEMENTS

(Total concentrations in micrograms per liter, except as indicated)

$\begin{array}{lr}\text { Arsenic (As) } & 0 \\ \text { Barium (Ba) } & 0 \\ \text { Cadmium (Cd) } & 0 \\ \text { Chromium (Cr) } & 10 \\ \text { Copper (Cu) } & 2 \\ \text { Iron (Fe) } & 20 \\ \text { Lead ( } \mathrm{Pb} \text { ) } & 6\end{array}$

$\begin{array}{lc}\text { Manganese (Mn) } & 0 \\ \text { Mercury ( } \mathrm{Hg}) & <\cdot 5 \\ \text { Selenium ( } \mathrm{Se}) & 0 \\ \text { Silver ( } \mathrm{Ag}) & 1 \\ \text { Strontium, dissolved ( } \mathrm{Sr}) & 140 \\ \text { Zinc ( } \mathrm{Zn}) & 10\end{array}$

ANALYSIS OF SELECTED PESTICIDE AND INDUSTRIAL COMPOUNDS

(Total concentrations in micrograms per 1iter)

$\begin{array}{llll}\text { Aldrin } & .00 & \text { Lindane } & .00 \\ \text { Chlordane } & .0 & \text { Methoxychlor } & .00 \\ \text { DDD } & .00 & \text { Mirex } & .00 \\ \text { DDE } & .00 & \text { PCB } & .0 \\ \text { DDT } & .00 & \text { PCN } & .00 \\ \text { Dieldrin } & .00 & \text { Silvex } & .00 \\ \text { Endrin } & .00 & \text { Toxaphene } & 0 \\ \text { Heptachlor } & .00 & 2,4-D & .00 \\ \text { Heptachlor epoxide } & .00 & 2,4,5-\mathrm{T} & .00\end{array}$

ANALYSIS OF SELECTED GROSS RADIOACTIVITY

(Total activity concentrations in picocuries per liter)

Gross Alpha, as uranium natural

Gross Beta, as

cesium-137
Gross Beta, as

strontium -90 
PUBLIC WATER SUPPLY: Jacksonville

COUNTY: Duval

COLLECTION DATE: 12-13-77

SAMPLING POINT (1) Treated water-301922081380301, fire hydrant north of Hart Bridge at Commodore Point.

(2) Raw water-

ANALYSIS OF MAJOR CHEMICAL CONSTITUENTS AND PHYSICAL PROPERTIES (Dissolved concentrations in milligrams per liter, except as indicated)

(1) (2)

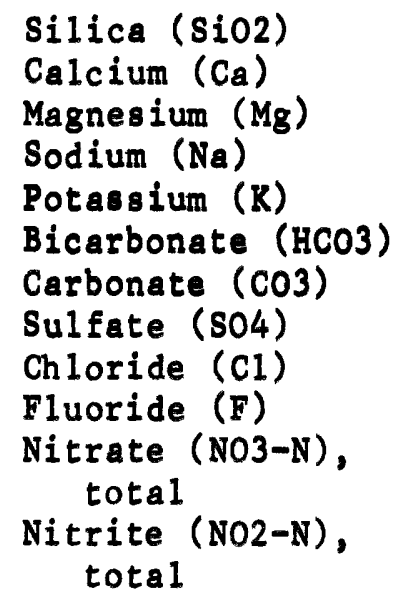

Dissolved solids (residue at $180^{\circ} \mathrm{C}$ )

Hardness as $\mathrm{CaCO} 3$ $(\mathrm{Ca}, \mathrm{Mg})$

Noncarbonate hardness as $\mathrm{CaCO} 3$

Percent sodium

Alkalinity as $\mathrm{CaCO} 3$

Specific conductance (umhos $/ \mathrm{cm}$ at $25^{\circ} \mathrm{C}$ )

$\mathrm{pH}$ (units)

Temperature $\left({ }^{\circ} \mathrm{C}\right)$

Color (Pt-Co units)

Turbidity (NTU)

(1)

ANALYSIS OF SELECTED TRACE ELEMENTS

(Total concentrations in micrograms per liter, except as indicated)

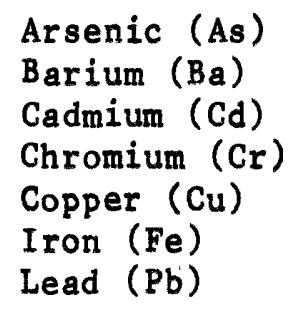

Manganese $(\mathrm{Mn})$

Mercury ( $\mathrm{Hg}$ )

Selenium ( $\mathrm{Se}$ )

Silver $(\mathrm{Ag})$

Strontium, dissolved ( $S r)$

Zinc $(\mathrm{Zn})$

ANALYSIS OF SELECTED PESTICIDE AND INDUSTRIAL COMPOUNDS

(Total concentrations in micrograms per liter)
Aldrin
Chlordane
DDD
DDE
DDT
Dieldrin
Endrin
Heptachlor
Heptachlor epoxide

580

7.1

19.5 
PUBLIC WATER SUPPLY: Jacksonville Beach

COUNTY: Duva1

COLLECTION DATE: 12-12-77

SAMPLING POINT (1) Treated water-301713081233401, tap at side of fire station opposite water treatment plant on 1st Avenue.

(2) Raw water-

ANALYSIS OF MAJOR CHEMICAL CONSTITUENTS AND PHYSICAL PROPERTIES

(Dissolved concentrations in milligrams : per 1 iter, except as indicated)

Silica (SiO2)

Calcium ( $\mathrm{Ca}$ )

Magnesium ( $\mathrm{Mg}$ )

Sodium ( $\mathrm{Na})$

Potassium (K)

Bicarbonate ( $\mathrm{HCO} 3$ )

Carbonate ( $\mathrm{CO} 3)$

Sulfate (S04)

Chloride (C1)

Fluoride (F)

Nitrate (NO3-N),

total

Nitrite (NO2-N),

total
(2)

(1)
(1)
750

$\mathrm{pH}$ (units)

Temperature $\left({ }^{\circ} \mathrm{C}\right)$

Color (Pt-Co units)

Turbidity (NTU)

ANALYSIS OF SELECTED TRACE ELEMENTS

(Total concentrations in micrograms per liter, except as indicated)

Arsenic (As)

Barium ( $\mathrm{Ba}$ )

Cadmium ( $C d)$

Chromium ( $\mathrm{Cr}$ )

Copper $(\mathrm{Cu})$

Iron ( $\mathrm{Fe}$ )

Lead $(\mathrm{Pb})$
Manganese (Mn)

Mercury ( $\mathrm{Hg})$

Selenium ( $\mathrm{Se}$ )

Silver ( $\mathrm{Ag})$

Strontium, dissolved ( $S r$ )

Zinc $(\mathrm{Zn})$

ANALYSIS OF SELECTED PESTICIDE AND INDUSTRIAL COMPOUNDS (Total concentrations in micrograms per 1iter)

$\begin{array}{ll}\text { Aldrin } & \text { Lindane } \\ \text { Chlordane } & \text { Methoxychlor } \\ \text { DDD } & \text { Mirex } \\ \text { DDE } & \text { PCB } \\ \text { DDT } & \text { PCN } \\ \text { Dieldrin } & \text { Silvex } \\ \text { Endrin } & \text { Toxaphene } \\ \text { Heptachlor } & 2,4-D \\ \text { Heptachlor epoxide } & 2,4,5-\mathrm{T}\end{array}$

ANALYSIS OF SELECTED GROSS RADIOACTIVITY

(Total activity concentrations in picocuries per liter)

Gross Alpha, as uranium natural $<5.5$

Gross Beta, as

cesium-137
Gross Beta, as

strontium -90
7.2

22.0 
PUBLIC WATER SUPPLY: Jacksonville Suburban Utility COUNTY: Duval COLLECTION DATE: $12-13-77$

SAMPLING POINT (1) Treated water-302054081350902, tap on line coming from pumphouse at corner of Pine Sumit Drive East and Columbine Drive.

(2) Raw water-

ANALYSIS OF MAJOR CHEMICAL CONSTITUENTS AND PHYSICAL PROPERTIES (Dissolved concentrations in milligrams per liter, except as indicated)

(1)

$\begin{array}{lc}\text { Silica (SiO2) } & 26 \\ \text { Calcium (Ca) } & 66 \\ \text { Magnesium (Mg) } & 25 \\ \text { Sodium (Na) } & 15 \\ \text { Potassium (K) } & 2.0 \\ \text { Bicarbonate (HCO3) } & 160 \\ \text { Carbonate (CO3) } & 0 \\ \text { Sulfate (SO4) } & 100 \\ \text { Chloride (C1) } & 38 \\ \text { Fluoride (F) } & .6 \\ \text { Nitrate (NO3-N), } & \\ \quad \text { total (NO2-N), } & .01 \\ \text { Nitrite (NO2) } \\ \quad \text { total }\end{array}$

(2)

Dissolved solids

$$
\text { (residue at } 180^{\circ} \mathrm{C} \text { ) } 361
$$

Hardness as $\mathrm{CaCO} 3$

$$
(\mathrm{Ca}, \mathrm{Mg} \text { ) }
$$

270

Noncarbonate hardness as $\mathrm{CaCO} 3$

140

Percent sodium

Alkalinity as $\mathrm{CaCO} 3$

Specific conductance

(umhos $/ \mathrm{cm}$ at $25^{\circ} \mathrm{C}$ )

(1)
$\mathrm{pH}$ (units)

Temperature $\left({ }^{\circ} \mathrm{C}\right)$

Color (Pt-Co units)

Turbidity (NTU)
11

130

605

27.5

0

2

ANALYSIS OF SELECTED TRACE ELEMENTS

(Total concentrations in micrograms per liter, except as indicated)

$\begin{array}{lr}\text { Arsenic (As) } & 2 \\ \text { Barium (Ba) } & - \\ \text { Cadmium (Cd) } & 0 \\ \text { Chromium (Cr) } & 20 \\ \text { Copper (Cu) } & 20 \\ \text { Iron (Fe) } & 30 \\ \text { Lead (Pb) } & 9\end{array}$

$\begin{array}{lc}\text { Manganese (Mn) } & 10 \\ \text { Mercury (Hg) } & <.5 \\ \text { Selenium ( Se) } & 0 \\ \text { Silver (Ag) } & 1 \\ \text { Strontium, dissolved (Sr) } & 1600 \\ \text { Zinc (Zn) } & 10\end{array}$

ANALYSIS OF SELECTED PESTICIDE AND INDUSTRIAL COMPOUNDS

(Total concentrations in micrograms per liter)

$\begin{array}{llll}\text { Aldrin } & .00 & \text { Lindane } & .00 \\ \text { Chlordane } & .0 & \text { Methoxychlor } & .00 \\ \text { DDD } & .00 & \text { Mirex } & .00 \\ \text { DDE } & .00 & \text { PCB } & .0 \\ \text { DDT } & .00 & \text { PCN } & .00 \\ \text { Dieldrin } & .00 & \text { Silvex } & .00 \\ \text { Endrin } & .00 & \text { Toxaphene } & 0 \\ \text { Heptachlor } & .00 & 2,4-D & .00 \\ \text { Heptachlor epoxide } & .00 & 2,4,5-\mathrm{T} & .00\end{array}$

ANALYSIS OF SELECTED GROSS RADIOACTIVITY

(Total activity concentrations in picocuries per liter)

Gross Alpha, as uranium natural $<3.1$ Gross Beta, as cesium-137
Gross Beta, as

strontium-90

1.9 
PUBLIC WATER SUPPLY: Jan Phy1 Village

COUNTY: Polk

COLLECTION DATE: 12-15-77

SAMPLING POINT (1) Treated water-280041081463209, tap on holding tank outside pumphouse.

(2) Raw water-

ANALYSIS OF MAJOR CHEMICAL CONSTITUENTS AND PHYSICAL PROPERTIES (Dissolved concentrations in milligrams per liter, except as indicated)

Silica ( $\mathrm{SiO2}$ ) 19

Calcium ( $\mathrm{Ca}$ )

Magnesium ( $\mathrm{Mg})$

Sodium ( $\mathrm{Na})$

Potassium ( $K$ )

Bicarbonate ( $\mathrm{HCO} 3$ )

Carbonate ( $\mathrm{CO} 3$ )

Sulfate (S04)

Chloride (C1)

Fluoride (F)

Nitrate (NO3-N),

total

Nitrite (NO2-N),

total
(1)

19
43

8.0

7.6

1.6

160

0

1.8

17

.2

.02

.00
(2)

Dissolved solids

(residue at $180^{\circ} \mathrm{C}$ ) $\quad 186$

Hardness as $\mathrm{CaCO} 3$

$(\mathrm{Ca}, \mathrm{Mg})$

140

Noncarbonate hardness as $\mathrm{CaCO} 3$

(1)

(2)

9

Percent sodium

Alkalinity as $\mathrm{CaCO} 3$

130

Specific conductance

(umhos $/ \mathrm{cm}$ at $25^{\circ} \mathrm{C}$ )

$\mathrm{pH}$ (units)

Temperature $\left({ }^{\circ} \mathrm{C}\right)$

Color (Pt-Co units)

Turbidity (NTU)

ANALYSIS OF SELECTED TRACE ELEMENTS

(Total concentrations in micrograms per liter, except as indicated)

$\begin{array}{lrll}\text { Arsenic (As) } & 1 & \text { Manganese (Mn) } & 0 \\ \text { Barium (Ba) } & -- & \text { Mercury (Hg) } & <.5 \\ \text { Cadmium (Cd) } & 0 & \text { Selenium ( } \mathrm{Se}) & 0 \\ \text { Chromium (Cr) } & 10 & \text { Silver (Ag) } & 0 \\ \text { Copper (Cu) } & 17 & \text { Strontium, dissolved (Sr) } & 80 \\ \text { Iron (Fe) } & 90 & \text { Zinc ( } \mathrm{n} \text { ) } & 0 \\ \text { Lead (Pb) } & 8 & & \end{array}$

ANALYSIS OF SELECTED PESTICIDE AND INDUSTRIAL COMPOUNDS (Total concentrations in micrograms per liter)

$\begin{array}{llll}\text { Aldrin } & .00 & \text { Lindane } & .00 \\ \text { Chlordane } & .0 & \text { Methoxych1or } & .00 \\ \text { DDD } & .00 & \text { Mirex } & .00 \\ \text { DDE } & .00 & \text { PCB } & .0 \\ \text { DDT } & .00 & \text { PCN } & .00 \\ \text { Dieldrin } & .00 & \text { Silvex } & .00 \\ \text { Endrin } & .00 & \text { Toxaphene } & 0 \\ \text { Heptachlor } & .00 & 2,4-D & .00 \\ \text { Heptachlor epoxide } & .00 & 2,4,5-T & .00\end{array}$

ANALYSIS OF SELECTED GROSS RADIOACTIVITY

(Total activity concentrations in picocuries per liter)

Gross Alpha, as uranium natural 4.3

Gross Beta, as

Gross Beta, as

cesium-137

strontium-90

2.3 
PUBLIC WATER SUPPLY: Jennings

COUNTY: Hamilton COLLECTION DATE: 12-08-77

SAMPLING POINT (1) Treated water-303611083054890, tap on southeast side of U.S. Post Office building.

(2) Raw water-

ANALYSIS OF MAJOR CHEMICAL CONSTITUENTS AND PHYSICAL PROPERTIES (Dissolved concentrations in milligrams per 1iter, except as indicated)

(1) (2)

$\begin{array}{lc}\text { Silica (SiO2) } & 31 \\ \text { Calcium (Ca) } & 36 \\ \text { Magnesium (Mg) } & 10 \\ \text { Sodium (Na) } & 3.4 \\ \text { Potassium (K) } & .9 \\ \text { Bicarbonate (HCO3) } & 150 \\ \text { Carbonate (CO3) } & 0 \\ \text { Sulfate (SO4) } & 8.2 \\ \text { Chloride (C1) } & 6.3 \\ \text { Fluoride (F) } & .3 \\ \text { Nitrate (NO3-N), } & \\ \quad \text { total (NO2-N), } & .01 \\ \text { Nitrite (NO2-N) } & \\ \quad \text { total } & .00\end{array}$

(1)

Dissolved solids

(residue at $180^{\circ} \mathrm{C}$ ) $\quad 180$

Hardness as $\mathrm{CaCO} 3$

$$
(\mathrm{Ca}, \mathrm{Mg})
$$

Noncarbonate hardness as $\mathrm{CaCO} 3$
Percent sodium

Alkalinity as $\mathrm{CaCO} 3$

Specific conductance

(umhos $/ \mathrm{cm}$ at $25^{\circ} \mathrm{C}$ )

$\mathrm{pH}$ (units)

Temperature $\left({ }^{\circ} \mathrm{C}\right)$

Color (Pt-Co units)

Turbidity (NTU)
130

\section{8}

5

120

225

7.8

15.0

0

1

ANALYSIS OF SELECTED TRACE ELEMENTS

(Total concentrations in micrograms per liter, except as indicated)

$\begin{array}{lrlc}\text { Arsenic (As) } & 0 & \text { Manganese (Mn) } & 30 \\ \text { Barium (Ba) } & 0 & \text { Mercury (Hg) } & <.5 \\ \text { Cadmium (Cd) } & 0 & \text { Selenium (Se) } & 0 \\ \text { Chromium (Cr) } & <10 & \text { Silver (Ag) } & 0 \\ \text { Copper (Cu) } & 10 & \text { Strontium, dissolved (Sr) } & 60 \\ \text { Iron (Fe) } & 20 & \text { Zinc (Zn) } & 390 \\ \text { Lead (Pb) } & 17 & & \end{array}$

ANALYSIS OF SELECTED PESTICIDE AND INDUSTRIAL COMPOUNDS (Total concentrations in micrograms per liter)

$\begin{array}{llll}\text { Aldrin } & .00 & \text { Lindane } & .00 \\ \text { Chlordane } & .0 & \text { Methoxychlor } & .00 \\ \text { DDD } & .00 & \text { Mirex } & .00 \\ \text { DDE } & .00 & \text { PCB } & .0 \\ \text { DDT } & .00 & \text { PCN } & .00 \\ \text { Dieldrin } & .00 & \text { Silvex } & .00 \\ \text { Endrin } & .00 & \text { Toxaphene } & .00 \\ \text { Heptachlor } & .00 & 2,4-D & .00\end{array}$

ANALYSIS OF SELECTED GROSS RADIOACTIVITY

(Total activity concentrations in picocuries per liter)

Gross Alpha, as uranium natural $\quad 5.6$

Gross Beta, as

cesium-137

1.0
Gross Beta, as

strontium-90 
PUBLIC WATER SUPPLY: Keaton Beach

COUNTY: Taylor

COLLECTION DATE: $11-28-77$

SAMPLING POINT (1) Treated water-295020083350090, tap outside on south side of gasoline station (combined with grocery store and Keaton Beach Marina).

(2) Raw water-

ANALYSIS OF MAJOR CHEMICAL CONSTITUENTS AND PHYSICAL PROPERTIES (Dissolved concentrations in milligrams per 1iter, except as indicated)

$\begin{array}{lc}\text { Silica (SiO2) } & (1) \\ \text { Calcium (Ca) } & 4.4 \\ \text { Magnesium (Mg) } & 33 \\ \text { Sodium (Na) } & 12 \\ \text { Potassium (K) } & 2.8 \\ \text { Bicarbonate (HCO3) } & .4 \\ \text { Carbonate (CO3) } & 0 \\ \text { Sulfate (SO4) } & .7 \\ \text { Chloride (C1) } & 3.4 \\ \text { Fluoride (F) } & .1 \\ \text { Nitrate (NO3-N), } \\ \quad \text { total (NO2-N), } \\ \text { Nitrite } \\ \quad \text { tota1 }\end{array}$

(1)

Dissolved solids (residue at $180^{\circ} \mathrm{C}$ ) $\quad 125$

Hardness as $\mathrm{CaCO} 3$ ( $\mathrm{Ca}, \mathrm{Mg}$ )

Noncarbonate hardness as $\mathrm{CaCO} 3$

Percent sodium

Alkalinity as $\mathrm{CaCO} 3 \quad 120$

3.7

Specific conductance (umhos $/ \mathrm{cm}$ at $25^{\circ} \mathrm{C}$ ) 262

pH (units)

Temperature $\left({ }^{\circ} \mathrm{C}\right)$

19.0

Color (Pt-Co units)

6

Turbidity (NTU)

3

ANALYSIS OF SELECTED TRACE ELEMENTS

(Total concentrations in micrograms per liter, except as indicated)

$\begin{array}{lr}\text { Arsenic (As) } & 1 \\ \text { Barium (Ba) } & 0 \\ \text { Cadmium ( } \mathrm{Cd} \text { ) } & 1 \\ \text { Chromium ( } \mathrm{Cr} \text { ) } & 10 \\ \text { Copper (Cu) } & 18 \\ \text { Iron (Fe) } & 260 \\ \text { Lead ( } \mathrm{Pb} \text { ) } & 14\end{array}$

Manganese $(\mathrm{Mn})$

10

$0 \quad$ Mercury ( $\mathrm{Hg}$ )

Selenium (Se)

Silver ( $\mathrm{Ag})$

Strontium, dissolved ( $\mathrm{Sr}$ ) 40

Zinc $(\mathrm{Zn})$

10

ANALYSIS OF SELECTED PESTICIDE AND INDUSTRIAL COMPOUNDS

(Total concentrations in micrograms per 1iter)

$\begin{array}{llll}\text { Aldrin } & .00 & \text { Lindane } & .00 \\ \text { Chlordane } & .0 & \text { Methoxychlor } & .00 \\ \text { DDD } & .00 & \text { Mirex } & .00 \\ \text { DDE } & .00 & \text { PCB } & .0 \\ \text { DDT } & .00 & \text { PCN } & .00 \\ \text { Dieldrin } & .00 & \text { Silvex } & .00 \\ \text { Endrin } & .00 & \text { Toxaphene } & 0 \\ \text { Heptachlor } & .00 & 2,4-D & .00 \\ \text { Heptachlor epoxide } & .00 & 2,4,5-\mathrm{T} & .00\end{array}$

ANALYSIS OF SELECTED GROSS RADIOACTIVITY

(Total activity concentrations in picocuries per liter)

Gross Alpha, as

uranium natural $<1.6$

Gross Beta, as

cesium-137
Gross Beta, as

strontium -90
$<.6$ 
PUBLIC WATER SUPPLY: Lake Alfred

COUNTY: Polk

COLLECTION DATE: $12-14-77$

SAMPLING POINT (1) Treated water-280537081434609, tap inside water treatment plant (we11 number 2) on south side.

(2) Raw water-

ANALYSIS OF MAJOR CHEMICAL CONSTITUENTS AND PHYSICAL PROPERTIES

(Dissolved concentrations in milligrams per liter, except as indicated)

$\begin{array}{lc}\text { Silica (SiO2) } & 14 \\ \text { Calcium (Ca) } & 40 \\ \text { Magnesium (Mg) } & 11 \\ \text { Sodium (Na) } & 8.6 \\ \text { Potassium (K) } & 2.2 \\ \text { Bicarbonate (HCO3) } & 140 \\ \text { Carbonate (CO3) } & 0 \\ \text { Sulfate (SO4) } & 16 \\ \text { Chloride (C1) } & 18 \\ \text { Fluoride (F) } & .1 \\ \text { Nitrate (NO3-N), } & \\ \quad \text { tota1 } & 2.4 \\ \text { Nitrite (NO2-N), } & \\ \quad \text { total }\end{array}$

(1) (2)
(1)

Dissolved solids

(residue at $180^{\circ} \mathrm{C}$ )

188

Hardness as $\mathrm{CaCO} 3$

$(\mathrm{Ca}, \mathrm{Mg})$

150

Noncarbonate hardness

as $\mathrm{CaCO} 3$

30

Percent sodium

Alkalinity as $\mathrm{CaCO} 3$

Specific conductance

(umhos $/ \mathrm{cm}$ at $25^{\circ} \mathrm{C}$ )

$\mathrm{pH}$ (units)

Temperature $\left({ }^{\circ} \mathrm{C}\right)$

Color (Pt-Co units)

Turbidity (NTU)

ANALYSIS OF SELECTED TRACE ELEMENTS

(Tota1 concentrations in micrograms per liter, except as indicated)

$\begin{array}{lrlc}\text { Arsenic (As) } & 2 & \text { Manganese (Mn) } & 0 \\ \text { Barium (Ba) } & - & \text { Mercury (Hg) } & <.5 \\ \text { Cadmium (Cd) } & 0 & \text { Selenium ( Se) } & 2 \\ \text { Chromium ( Cr) } & 20 & \text { Silver (Ag) } & 0 \\ \text { Copper (Cu) } & 5 & \text { Strontium, dissolved (Sr) } & 70 \\ \text { Iron (Fe) } & 30 & \text { Zinc (Zn) } & 20 \\ \text { Lead (Pb) } & 10 & & \end{array}$

ANALYSIS OF SELECTED PESTICIDE AND INDUSTRIAL COMPOUNDS ( Total concentrations in micrograms per liter)

$\begin{array}{llll}\text { A1drin } & .00 & \text { Lindane } & .00 \\ \text { Ch1ordane } & .0 & \text { Methoxych 1or } & .00 \\ \text { DDD } & .00 & \text { Mirex } & .00 \\ \text { DDE } & .00 & \text { PCB } & .0 \\ \text { DDT } & .00 & \text { PCN } & .00 \\ \text { Dieldrin } & .00 & \text { Silvex } & .00 \\ \text { Endrin } & .00 & \text { Toxaphene } & 0 \\ \text { Heptachlor } & .00 & 2,4-D & .00 \\ \text { Heptach1or epoxide } & .00 & 2,4,5-T & .00\end{array}$

ANALYSIS OF SELECTED GROSS RADIOACTIVITY

( Total activity concentrations in picocuries per liter)

Gross Alpha, as uranium natural

Gross Beta, as

cesium-137
Gross Beta, as

5.2

strontium-90

3.6 
PUBLIC WATER SUPPLY: Lake City

COUNTY: Columbia

COLLECTION DATE: $12-09-77$

SAMPLING POINT (1) Treated water-301108082372490, tap on front corner of gasoline station at corner of Seventh and Duval Streets.

(2) Raw water-

ANALYSIS OF MAJOR CHEMICAL CONSTITUENTS AND PHYSICAL PROPERTIES

(Dissolved concentrations in milligrams per liter, except as indicated)

Silica (SiO2)

Calcium ( $\mathrm{Ca}$ )

Magnesium $(\mathrm{Mg})$

Sodium $(\mathrm{Na})$

Potassium (K)

Bicarbonate ( $\mathrm{HCO} 3$ )

Carbonate ( $\mathrm{CO} 3$ )

Sulfate ( $\mathrm{SO} 4$ )

Chloride (C1)

Fluoride (F)

Nitrate $(\mathrm{NO}-\mathrm{N})$, total

Nitrite (NO2-N),

total
(2)

Dissolved solids

(residue at $180^{\circ} \mathrm{C}$ )

Hardness as $\mathrm{CaCO} 3$

$(\mathrm{Ca}, \mathrm{Mg})$

Noncarbonate hardness

as $\mathrm{CaCO}_{3}$

Percent sodium

Alkalinity as $\mathrm{CaCO} 3$

Specific conductance

(umhos $/ \mathrm{cm}$ at $25^{\circ} \mathrm{C}$ )

pH (units)

Temperature $\left({ }^{\circ} \mathrm{C}\right)$

Color (Pt-Co units)

Turbidity (NTU)

(1)

ANALYSIS OF SELECTED TRACE ELEMENTS

(Total concentrations in micrograms per liter, except as indicated)

Arsenic (As)

Barium ( $\mathrm{Ba}$ )

Cadmium ( $C d)$

Chromium ( $\mathrm{C} r$ )

Copper $(\mathrm{Cu})$

Iron ( $\mathrm{Fe}$ )

Lead $(\mathrm{Pb})$
Manganese (Mn)

Mercury ( $\mathrm{Hg}$ )

Selenium ( $\mathrm{Se}$ )

Silver $(\mathrm{Ag})$

Strontium, dissolved ( $S r$ )

Zine $(\mathrm{Zn})$
240

7.9

16.0

ANALYSIS OF SELECTED PESTICIDE AND INDUSTRIAL COMPOUNDS

(Total concentrations in micrograms per liter)

Aldrin

Chlordane

DDD

DDE

DDT

Dieldrin

Endrin

Heptach 1 or

Heptachlor epoxide
Lindane

Methoxych lor

Mirex

PCB

PCN

Silvex

Toxaphene

$2,4-D$

$2,4,5-T$

ANALYSIS OF SELECTED GROSS RADIOACTIVITY

(Total activity concentrations in picocuries per liter)

Gross Alpha, as

uranium natural $\quad 3.4$

Gross Beta, as

cesium- 137

1.2
Gross Beta, as

strontium-90 1.0 
PUBLIC WATER SUPPLY: Lee County Water System

COUNTY: Lee COLLECTION DATE: 02-03-78

SAMPLING POINT (1) Treated water-263824081513704, tap at Jamaica Bay West mobile home community (Ft. Meyers, surface water-ground water mixed).

(2) Raw water-

ANALYSIS OF MAJOR CHEMICAL CONSTITUENTS AND PHYSICAL PROPERTIES

(Dissolved concentrations in milligrams per liter, except as indicated)

ANALYSIS OF SELECTED TRACE ELEMENTS

(Total concentrations in micrograms per liter, except as indicated)

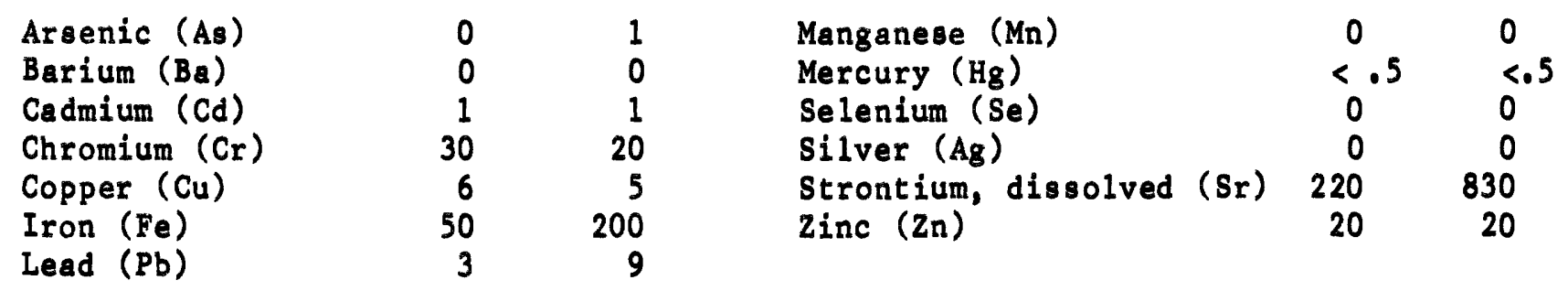

ANALYSIS OF SELECTED PESTICIDE AND INDUSTRIAL COMPOUNDS (Total concentrations in micrograms per liter)

$\begin{array}{llllll}\text { Aldrin } & .00 & .00 & \text { Lindane } & .00 & .00 \\ \text { Chlordane } & .0 & .0 & \text { Methoxychlor } & .00 & .00 \\ \text { DDD } & .00 & .00 & \text { Mirex } & .00 & .00 \\ \text { DDE } & .00 & .00 & \text { PCB } & .0 & .0 \\ \text { DDT } & .00 & .00 & \text { PCN } & .00 & .00 \\ \text { Dieldrin } & .00 & .00 & \text { Silvex } & .00 & .05 \\ \text { Endrin } & .00 & .00 & \text { Toxaphene } & 0 & 0 \\ \text { Heptachlor } & .00 & .00 & 2,4-D & .00 & .00 \\ \text { Heptachlor epoxide } & .00 & .00 & 2,4,5-T & .00 & .00\end{array}$

ANALYSIS OF SELECTED GROSS RADIOACTIVITY

(Total activity concentrations in picocuries per liter)

Gross Alpha, as uranium natural

Gross Beta, as

cesium -137
$5.6<4.2$

8.59 .2
Gross Beta, 28

$$
\text { strontium-90 }
$$

7.4

8.2 
PUBLIC WATER SUPPLY: Longwood

COUNTY: Seminole

COLLECTION DATE: 11-02-77

SAMPLING POINT (1) Treated water-284202081204401, tap inside power shed for water treatment plant near city buildings.

(2) Raw water-

ANALYSIS OF MAJOR CHEMICAL CONSTITUENTS AND PHYSICAL PROPERTIES

(Dissolved concentrations in milligrams per liter, except as indicated)

(1) (2)

11

33

Calcium (Ca)

Magnesium ( $\mathrm{Mg})$

Sodium ( $\mathrm{Na}$ )

Potassium ( $K$ )

Bicarbonate ( $\mathrm{HCO} 3)$

Carbonate ( $\mathrm{CO} 3)$

Sulfate (S04)

Chloride (C1)

Fluoride (F)

Nitrate (NO3-N), total

Nitrite (NO2-N), total

9.5
7.4
1.1
140
0
.8
9.8
.1
.00
.00

Dissolved solids

(residue at $180^{\circ} \mathrm{C}$ )

Hardness as $\mathrm{CaCO} 3$

$(\mathrm{Ca}, \mathrm{Mg}) \quad 120$

Noncarbonate hardness as $\mathrm{CaCO} 3$

(1)

139

Percent sodium

Alkalinity as $\mathrm{CaCO} 3$

Specific conductance

(umhos $/ \mathrm{cm}$ at $25^{\circ} \mathrm{C}$ )

$\mathrm{pH}$ (units)

Temperature $\left({ }^{\circ} \mathrm{C}\right)$

Color (Pt-Co units)

Turbidity (NTU)

ANALYSIS OF SELECTED TRACE ELEMENTS

(Total concentrations in micrograms per liter, except as indicated)

$\begin{array}{lr}\text { Arsenic (As) } & 0 \\ \text { Barium (Ba) } & 0 \\ \text { Cadmium (Cd) } & 0 \\ \text { Chromium (Cr) } & 10 \\ \text { Copper (Cu) } & 4 \\ \text { Iron (Fe) } & 40 \\ \text { Lead (Pb) } & 1\end{array}$

Manganese (Mn)

10

Mercury ( $\mathrm{Hg})$

$<.5$

Selenium ( $\mathrm{Se}$ )

0

Silver (Ag)

0

Strontium, dissolved ( $S r) \quad 150$

Zinc $(\mathrm{Zn})$

40

ANALYSIS OF SELECTED PESTICIDE AND INDUSTRIAL COMPOUNDS

(Total concentrations in micrograms per liter)

$\begin{array}{llll}\text { Aldrin } & .00 & \text { Lindane } & .00 \\ \text { Chlordane } & .0 & \text { Methoxychlor } & .00 \\ \text { DDD } & .00 & \text { Mirex } & .00 \\ \text { DDE } & .00 & \text { PCB } & .0 \\ \text { DDT } & .00 & \text { PCN } & .00 \\ \text { Dieldrin } & .00 & \text { Silvex } & .00 \\ \text { Endrin } & .00 & \text { Toxaphene } & 0 \\ \text { Heptachlor } & .00 & 2,4-D & .00 \\ \text { Heptachlor epoxide } & .00 & 2,4,5-T & .00\end{array}$

ANALYSIS OF SELECTED GROSS RADIOACTIVITY

(Total activity concentrations in picocuries per liter)

Gross Alpha, as uranium natural

Gross Beta, as

cesium-137
Gross Beta, as

strontium -90 
PUBLIC WATER SUPPLY: Lynn Haven

COUNTY: Bay

COLLECTION DATE: 12-22-77

SAMPLING POINT (1) Treated water-301429085385590, tap near City Commission Meeting Room door on east side of fire department building.

(2) Raw water-

ANALYSIS OF MAJOR CHEMICAL CONSTITUENTS AND PHYSICAL PROPERTIES

(Dissolved concentrations in milligrams per liter, except as indicated)

$\begin{array}{lc}\text { Silica ( } & 12 \\ \text { Calcium (Ca) } & 28 \\ \text { Magnesium (Mg) } & 18 \\ \text { Sodium (Na) } & 30 \\ \text { Potassium (K) } & 2.4 \\ \text { Bicarbonate (HCO3) } & 150 \\ \text { Carbonate (CO3) } & 0 \\ \text { Sulfate (SO4) } & 18 \\ \text { Chloride (C1) } & 52 \\ \text { Fluoride (F) } & .4 \\ \text { Nitrate (NO3-N), } & \\ \quad \text { total NO2-N), } \\ \text { Nitrite (NO2-N) } \\ \quad \text { total }\end{array}$

(2)

(1)

Dissolved solids

$$
\text { (residue at } 180^{\circ} \mathrm{C} \text { ) }
$$

225

Hardness as $\mathrm{CaCO} 3$ $(\mathrm{Ca}, \mathrm{Mg}$ )

Noncarbonate hardness as $\mathrm{CaCO} 3$

Percent sodium

31

Alkalinity as $\mathrm{CaCO} 3 \quad 120$

Specific conductance

(umhos $/ \mathrm{cm}$ at $25^{\circ} \mathrm{C}$ )

360

$\mathrm{pH}$ (units)

7.4

Temperature $\left({ }^{\circ} \mathrm{C}\right)$

Color (Pt-Co units)

17.0

Turbidity (NTU)

ANALYSIS OF SELECTED TRACE ELEMENTS

(Total concentrations in micrograms per liter, except as indicated)

$\begin{array}{lrlr}\text { Arsenic (As) } & 2 & \text { Manganese (Mn) } & 0 \\ \text { Barium (Ba) } & -- & \text { Mercury (Hg) } & <.5 \\ \text { Cadmium (Cd) } & 1 & \text { Selenium (Se) } & 0 \\ \text { Chromium (Cr) } & 10 & \text { Silver (Ag) } & 0 \\ \text { Copper (Cu) } & 5 & \text { Strontium, dissolved (Sr) } 6700 \\ \text { Iron (Fe) } & 70 & \text { Zinc (Zn) } & 50 \\ \text { Lead (Pb) } & 9 & & \end{array}$

ANALYSIS OF SELECTED PESTICIDE AND INDUSTRIAL COMPOUNDS (Total concentrations in micrograms per liter)

$\begin{array}{llll}\text { Aldrin } & .00 & \text { Lindane } & .00 \\ \text { Chlordane } & .0 & \text { Methoxych1or } & .00 \\ \text { DDD } & .00 & \text { Mirex } & .00 \\ \text { DDE } & .00 & \text { PCB } & .0 \\ \text { DDT } & .00 & \text { PCN } & .00 \\ \text { Dieldrin } & .00 & \text { Silvex } & .00 \\ \text { Endrin } & .00 & \text { Toxaphene } & 0 \\ \text { Heptachlor } & .00 & 2,4-D & .00 \\ \text { Heptachlor epoxide } & .00 & 2,4,5-T & .00\end{array}$

ANALYSIS OF SELECTED GROSS RADIOACTIVITY

(Total activity concentrations in picocuries per liter)

Gross Alpha, as
uranium natural
Gross Beta, as
cesium-137

Gross Beta, as

strontium -90 
PUBLIC WATER SUPPLY: Manalapan

COUNTY: Palm Beach

COLLECTION DATE: 12-06-77

SAMPLING POINT (1) Treated water-263422080031102, tap at south end of is land $1 / 4 \mathrm{mile}$ north of Bayton Inlet at west edge of road near fire hydrant.

(2) Rạw water-

ANALYSIS OF MAJOR CHEMICAL CONSTITUENTS AND PHYSICAL PROPERTIES

(Dissolved concentrations in milligrams per liter, except as indicated)

$\begin{array}{lc}\text { Silica (SiO2) } & 9.0 \\ \text { Calcium (Ca) } & 87 \\ \text { Magnesium (Mg) } & 2.0 \\ \text { Sodium (Na) } & 20 \\ \text { Potassium (K) } & 1.0 \\ \text { Bicarbonate (HCO3) } & 256 \\ \text { Carbonate (CO3) } & 0 \\ \text { Sulfate (SO4) } & 7.1 \\ \text { Chloride (C1) } & 41 \\ \text { Fluoride (F) } & .2 \\ \text { Nitrate (NO3-N), } & \\ \quad \text { total (NO2-N), } & .01 \\ \text { Nitrite } \quad & \\ \quad \text { total }\end{array}$

(2)

(1)

Dissolved solids

$$
\text { (residue at } 180^{\circ} \mathrm{C} \text { ) } 298
$$

Hardness as $\mathrm{CaCO} 3$

$(\mathrm{Ca}, \mathrm{Mg})$

Noncarbonate hardness

as $\mathrm{CaCO} 3 \quad 20$

Percent sodium 16

Alkalinity as $\mathrm{CaCO} 3 \quad 210$

Specific conductance

(umhos $/ \mathrm{cm}$ at $25^{\circ} \mathrm{C}$ ) $\quad 480$

$\mathrm{pH}$ (units)

7.4

Temperature $\left({ }^{\circ} \mathrm{C}\right)$

Color (Pt-Co units)

25.0

Turbidity (NTU)

ANALYSIS OF SELECTED TRACE ELEMENTS

( Total concentrations in micrograms per liter, except as indicated)

$\begin{array}{lr}\text { Arsenic (As) } & 0 \\ \text { Barium (Ba) } & 0 \\ \text { Cadmium ( } \mathrm{Cd} \text { ) } & 1 \\ \text { Chromium ( Cr) } & 10 \\ \text { Copper (Cu) } & 142 \\ \text { Iron (Fe) } & 470 \\ \text { Lead (Pb) } & 22\end{array}$

$\begin{array}{lc}\text { Manganese (Mn) } & 10 \\ \text { Mercury (Hg) } & <.5 \\ \text { Selenium ( Se) } & 0 \\ \text { Silver (Ag) } & 1 \\ \text { Strontium, dissolved ( } \mathrm{Sr}) & 700 \\ \text { Zinc ( } \mathrm{Zn}) & 10\end{array}$

ANALYSIS OF SELECTED PESTICIDE AND INDUSTRIAL COMPOUNDS

( Total concentrations in micrograms per 1 iter)

$\begin{array}{llll}\text { Aldrin } & .00 & \text { Lindane } & .00 \\ \text { Chlordane } & .0 & \text { Methoxych lor } & .00 \\ \text { DDD } & .00 & \text { Mirex } & .00 \\ \text { DDE } & .00 & \text { PCB } & .0 \\ \text { DDT } & .00 & \text { PCN } & .00 \\ \text { Dieldrin } & .00 & \text { Silvex } & .00 \\ \text { Endrin } & .00 & \text { Toxaphene } & 0 \\ \text { Heptachlor } & .00 & 2,4-D & .00 \\ \text { Heptachlor epoxide } & .00 & 2,4,5-\mathrm{T} & .00\end{array}$

ANALYSIS OF SELECTED GROSS RADIOACTIVITY

(Total activity concentrations in picocuries per liter)

\begin{tabular}{|c|c|}
\hline $\begin{array}{l}\text { Gross Alpha, as } \\
\text { uranium natural }\end{array}$ & 2 \\
\hline $\begin{array}{c}\text { Gross Beta, as } \\
\text { cesium- } 137\end{array}$ & 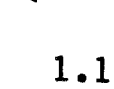 \\
\hline
\end{tabular}

Gross Beta, as

strontium -90 
PUBLIC WATER SUPPLY: Manatee County Water System COUNTY: Manatee COLLECTION DATE: 12-21-77

SAMPLING POINT (1) Treated water-272935082212009, water treatment plant, laboratory tap.

(2) Raw water-272935082212000, Lake Manatee intake southeast near Bradenton.

ANALYSIS OF MAJOR CHEMICAL CONSTITUENTS AND PHYSICAL PROPERTIES (Dissolved concentrations in milligrams per liter, except as indicated)

(1)

Silica $\left(\mathrm{SiO}_{2}\right)$
Calcium $(\mathrm{Ca})$
Magnesium $(\mathrm{Mg})$
Sodium $(\mathrm{Na})$
Potassium $(\mathrm{K})$
Bicarbonate $\left(\mathrm{HCO}_{3}\right)$
Carbonate $\left(\mathrm{CO}_{3}\right)$
Sulfate $\left(\mathrm{SO}_{4}\right)$
Chloride $(\mathrm{C1})$
Fluoride $(\mathrm{F})$
Nitrate $\left(\mathrm{NO}_{3}-\mathrm{N}\right)$,
total
Nitrite $\left(\mathrm{NO}_{2}-\mathrm{N}\right)$,
total

$5.4 \quad 5.8$

$29 \quad 7.7$

$4.8 \quad 4.6$

$6.1 \quad 5.7$

$2.9 \quad 2.9$

31

0

57

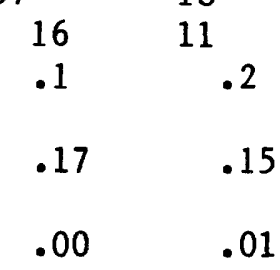

(1)

Dissolved solids

$$
\text { (residue at } 180^{\circ} \mathrm{C} \text { ) }
$$

141

Hardness as $\mathrm{CaCO}_{3}$

$$
\text { ( } \mathrm{Ca}, \mathrm{Mg} \text { ) }
$$

$$
93
$$

Noncarbonate hardness as $\mathrm{CaCO}_{3}$

\section{8}

17

Percent sodium

Alkalinity as $\mathrm{CaCO}_{3}$

Specific conductance

12

23

ANALYSIS OF SELECTED TRACE ELEMENTS (umhos $/ \mathrm{cm}$ at $25^{\circ} \mathrm{C}$ )

$\mathrm{pH}$ (units)

Temperature $\left({ }^{\circ} \mathrm{C}\right)$

Color (Pt-Co units)

Turbidity (NTU)
$25 \quad 22$

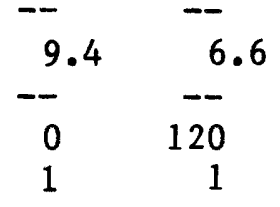

(Total concentrations in micrograms per liter, except as indicated)

$\begin{array}{lrrlrr}\text { Arsenic (As) } & 1 & 0 & \text { Manganese (Mn) } & 10 & 10 \\ \text { Barium (Ba) } & - & -- & \text { Mercury (Hg) } & <.5 & <.5 \\ \text { Cadmium (Cd) } & 0 & 0 & \text { Selenium (Se) } & 0 & 0 \\ \text { Chromium ( } \mathrm{Cr} \text { ) } & 40 & 10 & \text { Silver (Ag) } & 0 \\ \text { Copper (Cu) } & 3 & 8 & \text { Strontium, dissolved (Sr) } & 700 & 600 \\ \text { Iron (Fe) } & 20 & 330 & \text { Zinc (Zn) } & 100 & 30 \\ \text { Lead (Pb) } & 0 & 0 & & \end{array}$

ANALYSIS OF SELECTED PESTICIDE AND INDUSTRIAL COMPOUNDS (Total concentrations in micrograms per 1iter)

$\begin{array}{llllll}\text { Aldrin } & .00 & .00 & \text { Lindane } & .00 & .00 \\ \text { Chlordane } & .0 & .0 & \text { Methoxych 1or } & .00 & .00 \\ \text { DDD } & .00 & .00 & \text { Mirex } & .00 & .00 \\ \text { DDE } & .00 & .00 & \text { PCB } & .0 & .0 \\ \text { DDT } & .00 & .00 & \text { PCN } & .00 & .00 \\ \text { Dieldrin } & .00 & .00 & \text { Silvex } & .00 & .00 \\ \text { Endrin } & .00 & .00 & \text { Toxaphene } & 0 & 0 \\ \text { Heptachlor } & .00 & .00 & 2,4-D & .00 & .00 \\ \text { Heptachlor epoxide } & .00 & .00 & 2,4,5-T & .00 & .00\end{array}$

ANALYSIS OF SELECTED GROSS RADIOACTIVITY

(Total activity concentrations in picocuries per liter)

Gross Alpha, as uranium natural

Gross Beta, as

cesium-137
$1.9<1.2$

5.46 .0
Gross Beta, as

$$
\text { strontium }-90
$$

4.8

5.2 
PUBLIC WATER SUPPLY: Marco Island COLLECTION DATE: $11-29-77$ (treated) COUNTY: Collier

SAMPLING POINT (1) Treated water -255442081424401 , tap in 1aboratory of water treatment plant.

(2) Raw water-260351081414700, tap in mainland pipeline in yard of water treatment plant.

ANALYSIS OF MAJOR CHEMICAL CONSTITUENTS AND PHYSICAL PROPERTIES

(Dissolved concentrations in milligrams per liter, except as indicated)

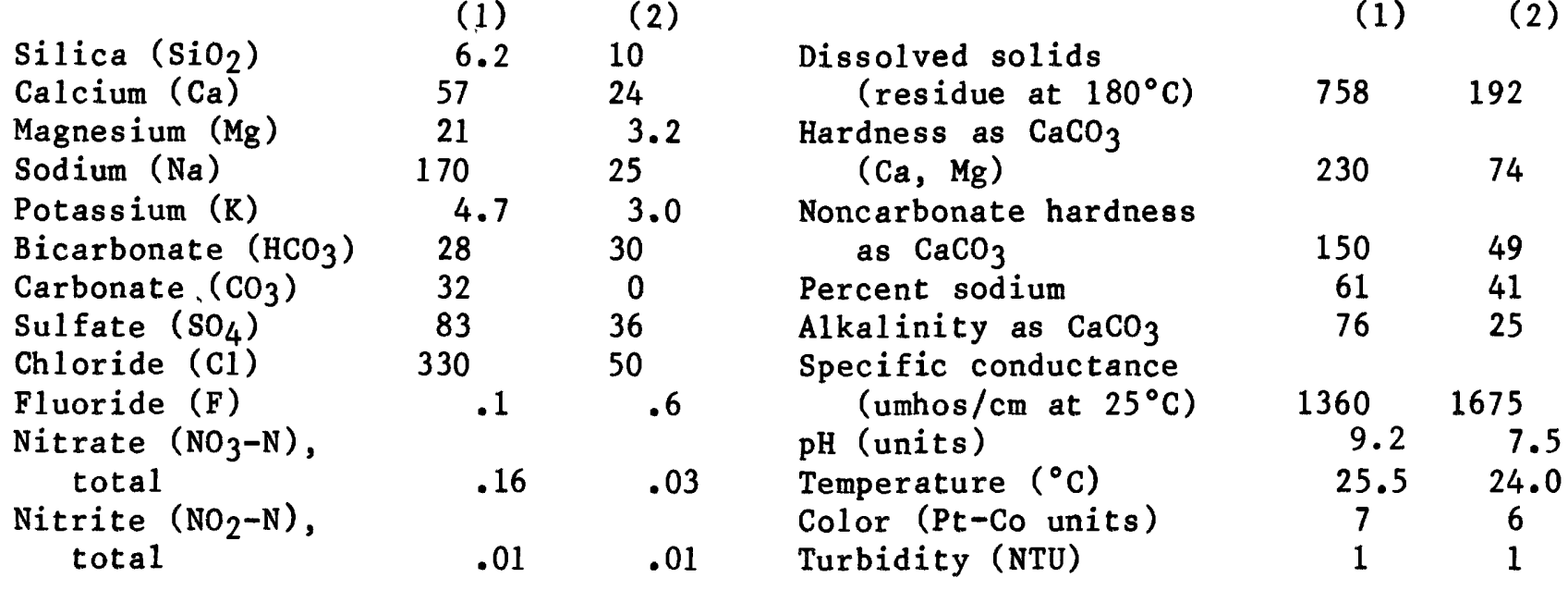

ANALYSIS OF SELECTED TRACE ELEMENTS

( Total concentrations in micrograms per liter, except as indicated)

$\begin{array}{lrrlrr}\text { Arsenic (As) } & 1 & 1 & \text { Manganese (Mn) } & 10 & 0 \\ \text { Barium (Ba) } & - & -- & \text { Mercury (Hg) } & <.5 & 0 \\ \text { Cadmium (Cd) } & 1 & 0 & \text { Selenium (Se) } & 0 \\ \text { Chromium ( Cr) } & 30 & 10 & \text { Silver (Ag) } & 0 & 0 \\ \text { Copper (Cu) } & 6 & 8 & \text { Strontium, dissolved (Sr) } & 380 & 340 \\ \text { Iron (Fe) } & 700 & 40 & \text { Zinc (Zn) } & 70 & 10 \\ \text { Lead (Pb) } & 30 & 13 & & & \end{array}$

ANALYSIS OF SELECTED PESTICIDE AND INDUSTRIAL COMPOUNDS

(Total concentrations in micrograms per liter)

\begin{tabular}{|c|c|c|c|c|}
\hline Aldrin & .00 & -- & Lindane & .00 \\
\hline Chlordane & .0 & -- & Methoxych lor & .00 \\
\hline DDD & .00 & -- & Mirex & .00 \\
\hline $\mathrm{DDE}$ & .00 & -- & PCB & .0 \\
\hline DDT & .00 & -- & PCN & .00 \\
\hline Dieldrin & .00 & -- & Silvex & .00 \\
\hline Endrin & .00 & - & Toxaphene & 0 \\
\hline Heptachlor & .00 & -- & $2,4-D$ & .00 \\
\hline Heptachlor epoxide & .00 & -- & $2,4,5-T$ & .00 \\
\hline
\end{tabular}

ANALYSIS OF SELECTED GROSS RADIOACTIVITY

(Total activity concentrations in picocuries per liter)

Gross Alpha, as

uranium natura1

Gross Beta, as

cesium-137
$19<2.0$

$6.6 \quad 2.5$
Gross Beta, as

strontium-90

5.9

2.0 
PUBLIC WATER SUPPLY: Melbourne

COUNTY: Brevard

COLLECTION DATE: 11-12-77

SAMPLING POINT (1) Treated water-02232100, tap at city of Melbourne water treatment plant.

(2) Raw water-02232100, Lake Washington.

ANALYSIS OF MAJOR CHEMICAL CONSTITUENTS AND PHYSICAL PROPERTIES

(Dissolved concentrations in milligrams per liter, except as indicated)

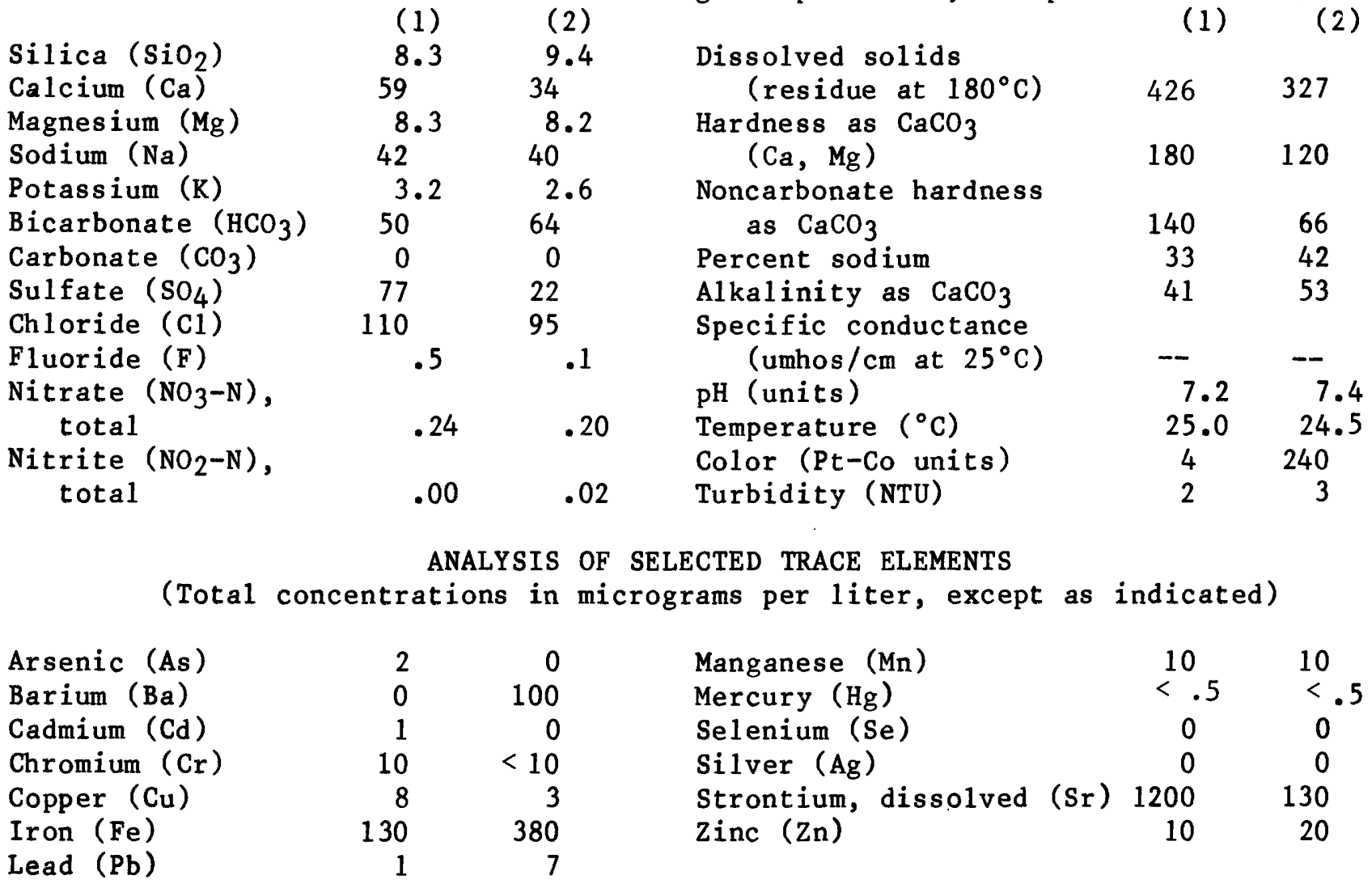

ANALYSIS OF SELECTED PESTICIDE AND INDUSTRIAL COMPOUNDS (Total concentrations in micrograms per liter)

$\begin{array}{llllll}\text { Aldrin } & .00 & .00 & \text { Lindane } & .00 & .00 \\ \text { Chlordane } & .0 & .0 & \text { Methoxychlor } & .00 & .00 \\ \text { DDD } & .00 & .00 & \text { Mirex } & .00 & .00 \\ \text { DDE } & .00 & .00 & \text { PCB } & .0 & .0 \\ \text { DDT } & .00 & .00 & \text { PCN } & .00 & .00 \\ \text { Dieldrin } & .00 & .00 & \text { Silvex } & .00 & .00 \\ \text { Endrin } & .00 & .00 & \text { Toxaphene } & 0 & 0 \\ \text { Heptachlor } & .00 & .00 & 2,4-D & .09 & .04 \\ \text { Heptachlor epoxide } & .00 & .00 & 2,4,5-T & .00 & .00\end{array}$

ANALYSIS OF SELECTED GROSS RADIOACTIVITY

(Total activity concentrations in picocuries per liter)

Gross Alpha, as uranium natural

Gross Beta, as

cesium-137
Gross Beta, as

$4.4<3.2$

strontium-90

$6.6 \quad 6.5$ 
PUBLIC. WATER SUPPLY; WEA:" kas?

COUNTY: BaY

COLLECTION DATE: $12-23-77$

SAMPLING POINT (1) Treefer uster-995645085243990, tap on front of pumphouse nent watse towat.

(2) naw vater.

ANALYSIS OF MA.MR CHEMICAL CONSTITUENTS AND PHYSICAL PROPERTIES

(Dissolved concentrations in milligrams per liter, except as indicated)

$\begin{array}{lr}\text { Silica (SiO2) } & (1) \\ \text { Calcium (Ca) } & 12 \\ \text { Magnesium (Mg) } & 24 \\ \text { Sodium (Na) } & 38 \\ \text { Potassium (K) } & 140 \\ \text { Bicarbonate (HCO3) } & 11 \\ \text { Carbonate (CO3) } & 270 \\ \text { Sulfate (SO4) } & 0 \\ \text { Chloride (C1) } & 160 \\ \text { Fluoride (F) } & 98 \\ \text { Nitrate (NO3-N), } & 3.3 \\ \quad \text { tota1 (NO2-N), } & .01 \\ \text { Nitrite (Notal } & \\ \quad \text { total } & .00\end{array}$

(2)

Dissolved solids

(residue at $180^{\circ} \mathrm{C}$ ) $\quad 610$

Hardness as $\mathrm{CaCO} 3$

$(\mathrm{Ca}, \mathrm{Mg}$ )

(1)

220

Noncarbonate hardness as $\mathrm{CaCO} 3$

Percent sodium

Alkalinity as $\mathrm{CaCO} 3$

Specific conductance

(umhos $/ \mathrm{cm}$ at $25^{\circ} \mathrm{C}$ )

pH (units)

Temperature $\left({ }^{\circ} \mathrm{C}\right)$

Color (Pt-Co units)

Turbidity (NTU)

ANALYSIS OF SELECTED TRACE ELEMENTS

(Total concentrations in micrograms per liter, except as indicated)

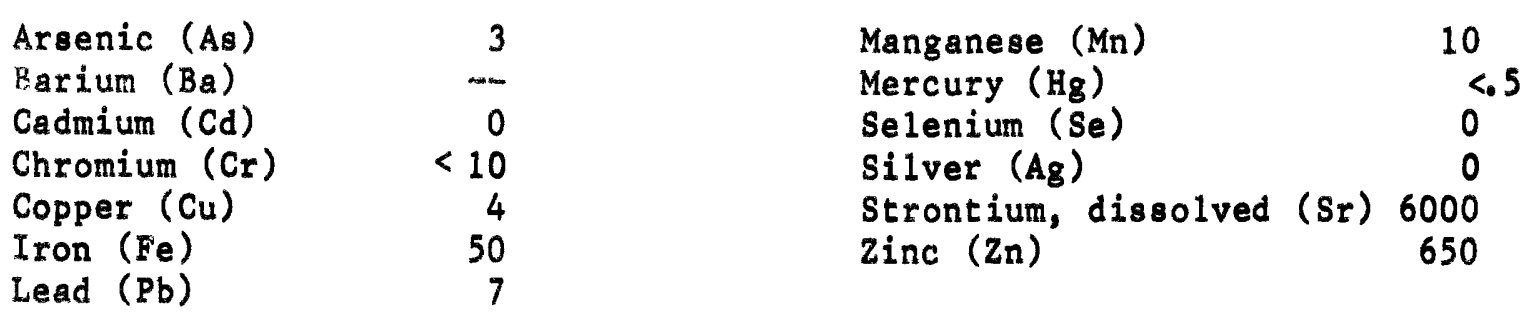

ANALYSIS OF SELECTED PESTICIDE AND INDUSTRIAL COMPOUNDS (Total concentrations in micrograms per 1 iter)

$\begin{array}{llll}\text { Aldrin } & .00 & \text { Lindane } & .00 \\ \text { Chlordane } & .0 & \text { Methoxychlor } & .00 \\ \text { DDD } & .00 & \text { Mirex } & .00 \\ \text { DDE } & .00 & \text { PCB } & .0 \\ \text { DDT } & .00 & \text { PCN } & .00 \\ \text { Dieldrin } & .00 & \text { Silvex } & .00 \\ \text { Endrin } & .00 & \text { Toxaphene } & 0 \\ \text { Heptachlor } & .00 & 2,4-D & .00 \\ \text { Heptachlor epoxide } & .00 & 2,4,5-T & .00\end{array}$

ANALYSIS OF SELECTED GROSS RADIOACTIVITY

(Total activity concentrations in picocuries per liter)

Gross Alpha, as

uranium natural

Gross Beta, as

cesium-137
Gross Beta, as

strontium-90 
PUBLIC WATER SUPPLY: Mims

COUNTY: Brevard

COLLECTION DATE: 11-02-77

SAMPLING POINT (1) Treated water-283931080514501, tap inside office of water treatment plant.

(2) Raw water-

ANALYSIS OF MAJOR CHEMICAL CONSTITUENTS AND PHYSICAL PROPERTIES (Dissolved concentrations in milligrams per liter, except 28 indicated)
(1)
(2)
(1)

7.3

Silica (SiO2)

Calcium ( $\mathrm{Ca}$ )

Magnesium ( $\mathrm{Mg}$ )

Sodium ( $\mathrm{Na}$ )

Potassium ( $K$ )

Bicarbonate ( $\left.\mathrm{HCO}_{3}\right)$

Carbonate ( $\mathrm{CO} 3$ )

Sulfate ( $\mathrm{SO4}$ )

Chloride (C1)

Fluoride (F)

Nitrate (NO3-N),

total

Nitrite (NO2-N),

total
93

6.2

29

1.4

250

\section{0}

51

54

.0

.23

.00
Dissolved solids

(residue at $180^{\circ} \mathrm{C}$ ) 395

Hardness as $\mathrm{CaCO} 3$

$(\mathrm{Ca}, \mathrm{Mg}) \quad 260$

Noncarbonate hardness

as $\mathrm{CaCO} 3$

Percent sodium 20

Alkalinity as $\mathrm{CaCO} 3 \quad 210$

Specific conductance

(umhos $/ \mathrm{cm}$ at $25^{\circ} \mathrm{C}$ )

$\mathrm{pH}$ (units)

Temperature $\left({ }^{\circ} \mathrm{C}\right)$

Color (Pt-Co units)

Turb idi y (NTU)

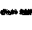

7.2

25.0

0

1

ANALYSIS OF SELECTED TRACE ELEMENTS

(Total concentrations in micrograms per liter, except as indicated)

$\begin{array}{lr}\text { Arsenic (As) } & 2 \\ \text { Barium (Ba) } & 0 \\ \text { Cadmium (Cd) r } & 0 \\ \text { Chromium (Cr) } & 10 \\ \text { Copper (Cu) } & 5 \\ \text { Iron (Fe) } & 30 \\ \text { Lead (Pb) } & 1\end{array}$

Manganese (Mn)

10

Mercury ( $\mathrm{Hg}$ )

Selenium ( $\mathrm{Se}$ )

$<.5$

0

Silver ( $\mathrm{Ag}$ )

Strontium, dissolved ( $\mathrm{Sr}$ ) 530

Zinc $(2 \pi)$

10

ANALYSIS OF SELECTED PESTICIDE AND INDUSTRIAL COMPOUNDS

(Total concentrations in micrograms per liter)

$\begin{array}{llll}\text { Aldrin } & .00 & \text { Lindane } & .00 \\ \text { Chlordane } & .0 & \text { Methoxychlor } & .00 \\ \text { DDD } & .00 & \text { Mirex } & .00 \\ \text { DDE } & .00 & \text { PCB } & .0 \\ \text { DDT } & .00 & \text { PCN } & .00 \\ \text { Dieldrin } & .00 & \text { Silvex } & .00 \\ \text { Endrin } & .00 & \text { Toxaphene } & 0 \\ \text { Heptachlor } & .00 & 2,4-D & .00 \\ \text { Heptachlor epoxide } & .00 & 2,4,5-\mathrm{T} & .00\end{array}$

ANALYSIS OF SELECTED GROSS RADIOACTIVITY

(Total activity concentrations in picocuries per liter)

Gross Alpha, as

uranium natural --

Gross Beta, as

cesium-137
Gross Beta, as

strontium-90 
PUBLIC WATER SUPPLY: ME. Dora

COUNTY: Lake

COLLECTION DATE: 11-02-77

SAMPLING POINT (1) Treated water-284856081383002, tap outside water treatment plant.

(2) Raw water-

ANALYSIS OF MAJOR CHEMICAL CONSTITUENTS AND PHYSICAL PROPERTIES (Dissolved concentrations in milligrams per liter, except as indicated)

$\begin{array}{lc}\text { Silica (SiO2) } & 9.6 \\ \text { Calcium (Ca) } & 34 \\ \text { Magnesium (Mg) } & 9.0 \\ \text { Sodium (Na) } & 7.0 \\ \text { Potassium (K) } & 2.6 \\ \text { Bicarbonate (HCO3) } & 130 \\ \text { Carbonate (CO3) } & 0 \\ \text { Sulfate (SO4) } & 13 \\ \text { Chloride (C1) } & 13 \\ \text { Fluoride (F) } & .1 \\ \text { Nitrate (NO3-N), } & \\ \quad \text { total (NO2-N), } & .06 \\ \text { Nitrite } \quad \\ \quad \text { total }\end{array}$

(2)

(1)

Dissolved solids

$$
\text { (residue at } 180^{\circ} \mathrm{C} \text { ) } \quad 137
$$

Hardness as $\mathrm{CaCO} 3$

$(\mathrm{Ca}, \mathrm{Mg}) \quad 120$

Noncarbonate hardness as $\mathrm{CaCO} 3 \quad 15$

Percent sodium 11

Alkalinity as $\mathrm{CaCO} 3 \quad 110$

Specific conductance

(umhos $/ \mathrm{cm}$ at $25^{\circ} \mathrm{C}$ ) 271

$\mathrm{pH}$ (units) 7.5

Temperature $\left({ }^{\circ} \mathrm{C}\right)$

Color (Pt-Co units) 12

Turbidity (NTU) 1

ANALYSIS OF SELECTED TRACE ELEMENTS

(Total concentrations in micrograms per liter, except as indicated)

$\begin{array}{lrlr}\text { Arsenic (As) } & 0 & \text { Manganese (Mn) } & 10 \\ \text { Barium (Ba) } & 0 & \text { Mercury (Hg) } & <.5 \\ \text { Cadmium (Cd) } & 0 & \text { Selenium ( Se) } & 0 \\ \text { Chromium (Cr) } & <10 & \text { Silver (Ag) } & 0 \\ \text { Copper (Cu) } & 3 & \text { Strontium, dissolved (Sr) } & 50 \\ \text { Iron (Fe) } & 40 & \text { Zinc (Zn) } & 130 \\ \text { Lead (Pb) } & 8 & & \end{array}$

ANALYSIS OF SELECTED PESTICIDE AND INDUSTRIAL COMPOUNDS

( Total concentrations in micrograms per liter)

$\begin{array}{llll}\text { Aldrin } & .00 & \text { Lindane } & .00 \\ \text { Chlordane } & .0 & \text { Methoxychlor } & .00 \\ \text { DDD } & .00 & \text { Mirex } & .0 \\ \text { DDE } & .00 & \text { PCB } & .00 \\ \text { DDT } & .00 & \text { PCN } & .00 \\ \text { Dieldrin } & .00 & \text { Silvex } & 0 \\ \text { Endrin } & .00 & \text { Toxaphene } & .00 \\ \text { Heptachlor } & .00 & 2,4-D & .00\end{array}$

ANALYSIS OF SELECTED GROSS RADIOACTIVITY

(Total activity concentrations in picocuries per liter)

Gross Alpha, as uranium natural --

Gross Beta, as

cesium-137
Gross Beta, as

strontium -90 
PUBLIC WATER SUPPLY: Mulberry

COUNTY: Polk

COLLECTION DATE: 12-20-77

SAMPLING POINT (1) Treated water-275338081581909, tap outside water treatment plant on north side.

(2) Raw water-

ANALYSIS OF MAJOR CHEMICAL CONSTITUENTS AND PHYSICAL PROPERTIES (Dissolved concentrations in milligrams per liter, except as indicated)

(1) (2)

$\begin{array}{lc}\text { Silica ( } & 20 \\ \text { Calcium (Ca) } & 44 \\ \text { Magnesium (Mg) } & 13 \\ \text { Sodium (Na) } & 6.6 \\ \text { Potassium (K) } & .9 \\ \text { Bicarbonate (HCO3) } & 180 \\ \text { Carbonate (CO3) } & 0 \\ \text { Sulfate (SO4) } & 7.8 \\ \text { Chloride (C1) } & 8.8 \\ \text { Fluoride (F) } & .3 \\ \text { Nitrate (NO3-N), } & \\ \quad \text { total (NO2-N), } & .00 \\ \text { Nitrite (NO2-N) } & .00 \\ \quad \text { total }\end{array}$

$\begin{array}{lr}\begin{array}{l}\text { Dissolved solids } \\ \quad \text { (residue at } 180^{\circ} \mathrm{C} \text { ) }\end{array} & 187 \\ \text { Hardness as CaCO3 } & \\ \quad \text { (Ca, Mg) } & 160 \\ \text { Noncarbonate hardness } & \\ \quad \text { as CaCO3 } & 16 \\ \text { Percent sodium } & 8 \\ \text { Alkalinity as CaC03 } & 150 \\ \begin{array}{l}\text { Specific conductance } \\ \quad \text { (umhos/cm at } 25^{\circ} \mathrm{C} \text { ) }\end{array} & -- \\ \text { pH (units) } & 7.4 \\ \left.\text { Temperature ( }{ }^{\circ} \mathrm{C}\right) & -- \\ \text { Color (Pt-Co units) } & 2 \\ \text { Turbidity (NTU) } & 1\end{array}$

(1)

ANALYSIS OF SELECTED TRACE ELEMENTS

(Total concentrations in micrograms per liter, except as indicated)

$\begin{array}{lrlr}\text { Arsenic (As) } & 2 & \text { Manganese (Mn) } & 10 \\ \text { Barium (Ba) } & -- & \text { Mercury (Hg) } & <.5 \\ \text { Cadmium (Cd) } & 1 & \text { Selenium (Se) } & 0 \\ \text { Chromium (Cr) } & <10 & \text { Silver (Ag) } & 2 \\ \text { Copper (Cu) } & 4 & \text { Strontium, dissolved (Sr) } & 510 \\ \text { Iron (Fe) } & 30 & \text { Zinc (Zn) } & 20 \\ \text { Lead (Pb) } & 15 & & \end{array}$

ANALYSIS OF SELECTED PESTICIDE AND INDUSTRIAL COMPOUNDS

(Total concentrations in micrograms per liter)

$\begin{array}{llll}\text { Aldrin } & .00 & \text { Lindane } & .00 \\ \text { Chlordane } & .0 & \text { Methoxychlor } & .00 \\ \text { DDD } & .00 & \text { Mirex } & .00 \\ \text { DDE } & .00 & \text { PCB } & .0 \\ \text { DDT } & .00 & \text { PCN } & .00 \\ \text { Dieldrin } & .00 & \text { Silvex } & .00 \\ \text { Endrin } & .00 & \text { Toxaphene } & 0 \\ \text { Heptach1or } & .00 & 2,4-D & .00 \\ \text { Heptachlor epoxide } & .00 & 2,4,5-T & .00\end{array}$

ANALYSIS OF SELECTED GROSS RADIOACTIVITY

(Total activity concentrations in picocuries per liter)

Gross Alpha, as uranium natural

Gross Beta, as

cesium-137
1.6

Gross Beta, as

strontium-90
.00

.00

.0

.00

.00

.00

.00 
PUBLIC WATER SUPPLY: Navarre Beach

COUNTY: Santa Rosa

COLLECTION DATE: 01-05-78

SAMPLING POINT (1) Treated water-302244086525290, tap on pier on west side of restaurant and tackle shop.

(2) Raw water-

ANALYSIS OF MAJOR CHEMICAL CONSTITUENTS AND PHYSICAL PROPERTIES (Dissolved concentrations in milligrams per liter, except as indicated)

(1)

$\begin{array}{lc}\text { Silica (SiO2) } & 12 \\ \text { Calcium (Ca) } & 3.1 \\ \text { Magnesium (Mg) } & 2.1 \\ \text { Sodium (Na) } & 190 \\ \text { Potassium (K) } & 6.0 \\ \text { Bicarbonate (HCO3) } & 260 \\ \text { Carbonate (CO3) } & 0 \\ \text { Sulfate (SO4) } & 13 \\ \text { Chloride (C1) } & 150 \\ \text { Fluoride (F) } & 1.0 \\ \text { Nitrate (NO3-N), } & \\ \quad \text { total (N2-N), } & .01 \\ \text { Nitrite (NO2-N), } & \\ \quad \text { total } & .02\end{array}$

(2)

Dissolved solids

(1) (residue at $180^{\circ} \mathrm{C}$ ) $\quad 491$

Hardness as $\mathrm{CaCO} 3$

$(\mathrm{Ca}, \mathrm{Mg}$ )

18

Noncarbonate hardness as $\mathrm{CaCO} 3$

Percent sodium

Alkalinity as $\mathrm{CaCO} 3$

Specific conductance

(umhos $/ \mathrm{cm}$ at $25^{\circ} \mathrm{C}$ )

$\mathrm{pH}$ (units)

Temperature $\left({ }^{\circ} \mathrm{C}\right)$

Color (Pt-Co units)

Turbidity (NTU)
0

95

210

745

7.5

11.0

6

4

ANALYSIS OF SELECTED TRACE ELEMENTS

(Total concentrations in micrograms per liter, except as indicated)

$\begin{array}{lrlr}\text { Arsenic (As) } & 1 & \text { Manganese (Mn) } & 0 \\ \text { Barium (Ba) } & -- & \text { Mercury (Hg) } & <.5 \\ \text { Cadmium (Cd) } & 1 & \text { Selenium (Se) } & 0 \\ \text { Chromium (Cr) } & <10 & \text { Silver (Ag) } & 0 \\ \text { Copper (Cu) } & 15 & \text { Strontium, dissolved (Sr) } 1200 \\ \text { Iron (Fe) } & 220 & \text { Zinc (Zn) } & 390 \\ \text { Lead (Pb) } & 52 & & \end{array}$

ANALYSIS OF SELECTED PESTICIDE AND INDUSTRIAL COMPOUNDS

(Total concentrations in micrograms per liter)

$\begin{array}{llll}\text { Aldrin } & .00 & \text { Lindane } & .00 \\ \text { Chlordane } & .0 & \text { Methoxychlor } & .00 \\ \text { DDD } & .00 & \text { Mirex } & .00 \\ \text { DDE } & .00 & \text { PCB } & .0 \\ \text { DDT } & .00 & \text { PCN } & .00 \\ \text { Dieldrin } & .00 & \text { Silvex } & .00 \\ \text { Endrin } & .00 & \text { Toxaphene } & 0 \\ \text { Heptachlor } & .00 & 2,4-D & .00 \\ \text { Heptachlor epoxide } & .00 & 2,4,5-T & .00\end{array}$

ANALYSIS OF SELECTED GROSS RADIOACTIVITY

(Total activity concentrations in picocuries per liter)

Gross Alpha, as

uranium natural

Gross Beta, as

cesium-137
Gross Beta, as

strontium-90 
PUBLIC WATER SUPPLY: Neptune Beach

COUNTY: Duval

COLLECTION DATE: 12-13-77

SAMPLING POINT (1) Treated water-301858081250401, fire hydrant on Kings Road, 0.1 miles north of Forest Road.

(2) Raw water-

ANALYSIS OF MAJOR CHEMICAL CONSTITUENTS AND PHYSICAL PROPERTIES

(Dissolved concentrations in milligrams per liter, except as indicated)

Silica (SiO2)

Calcium ( $\mathrm{Ca}$ )

Magnesium ( $\mathrm{Mg}$ )

Sodium ( $\mathrm{Na})$

Potassium (K)

Bicarbonate ( $\mathrm{HCO} 3$ )

Carbonate ( $\mathrm{CO} 3$ )

Sulfate (S04)

Chloride (C1)

Fluoride (F)

Nitrate (NO3-N), total

Nitrite ( NO2-N), total
(2)

Dissolved solids

(residue at $180^{\circ} \mathrm{C}$ )

Hardness as $\mathrm{CaCO} 3$

( $\mathrm{Ca}, \mathrm{Mg})$

Noncarbonate hardness

as $\mathrm{CaCO} 3$

Percent sodium

Alkalinity as $\mathrm{CaCO} 3$

Specific conductance

(umhos $/ \mathrm{cm}$ at $25^{\circ} \mathrm{C}$ )

$\mathrm{pH}$ (units)

630

Temperature $\left({ }^{\circ} \mathrm{C}\right)$

Color (Pt-Co units)

Turbidity (NTU)

(1)

ANALYSIS OF SELECTED TRACE ELEMENTS

(Total concentrations in micrograms per liter, except as indicated)

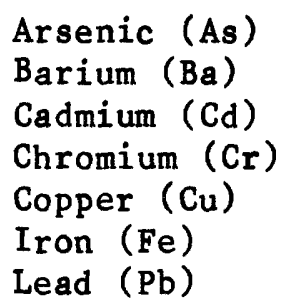

Lead $(\mathrm{Pb})$

Manganese $(\mathrm{Mn})$

Mercury ( $\mathrm{Hg}$ )

Selenium ( $\mathrm{Se}$ )

Silver ( $\mathrm{Ag}$ )

Strontium, dissolved ( $\mathrm{Sr}$ )

Zinc ( $\mathrm{Zn}$ )

ANALYSIS OF SELECTED PESTICIDE AND INDUSTRIAL COMPOUNDS

(Total concentrations in micrograms per 1iter)
Aldrin
Lind ane
Chlordane
Methoxych lor
DDD
Mirex
DDE
PCB
DDT
PCN
Dieldrin
Silvex
Endrin
Toxaphene
Heptach lor
2,4-D
Heptachlor epoxide
$2,4,5-T$

ANALYSIS OF SELECTED GROSS RADIOACTIVITY

(Total activity concentrations in picocuries per liter)

Gross Alpha, as

uranium natural $<4.0$

Gross Beta, as

cesium-137
Gross Beta, as

strontium-90

1.6 
PUBLIC WATER SUPPLY: New Smyrna Beach

COUNTY: Volusia

COLLECTION DATE: 12-05-77

SAMPLING POINT (1) Treated water-285953080575902, tap in back outside of water treatment plant.

(2) Raw water-

ANALYSIS OF MAJOR CHEMICAL CONSTITUENTS AND PHYSICAL PROPERTIES (Dissolved concentrations in milligrams per liter, except as indicated)

(1) (2)

$\begin{array}{lc}\text { Silica ( } & 19 \\ \text { Calcium ( } \mathrm{Ca}) & 25 \\ \text { Magnesium (Mg) } & 7.6 \\ \text { Sodium (Na) } & 35 \\ \text { Potassium (K) } & 1.7 \\ \text { Bicarbonate (HCO3) } & 68 \\ \text { Carbonate (CO3) } & 0 \\ \text { Sulfate (SO4) } & 1.5 \\ \text { Chloride (C1) } & 76 \\ \text { Fluoride (F) } & .1 \\ \text { Nitrate (NO3-N), } & \\ \quad \text { total (NO2-N), } \\ \text { Nitrite } \quad \\ \quad \text { total }\end{array}$

(1)

Dissolved solids

$$
\text { (residue at } 180^{\circ} \mathrm{C} \text { ) } 217
$$

Hardness as $\mathrm{CaCO} 3$

$$
\text { ( } \mathrm{Ca}, \mathrm{Mg} \text { ) } 94
$$

Noncarbonate hardness as $\mathrm{CaCO} 3$

Percent sodium 44

Alkalinity as $\mathrm{CaCO} 3 \quad 56$

Specific conductance

(umhos $/ \mathrm{cm}$ at $25^{\circ} \mathrm{C}$ )

$\mathrm{pH}$ (units)

Temperature $\left({ }^{\circ} \mathrm{C}\right)$

Color (Pt-Co units)

Turbidity (NTU)

ANALYSIS OF SELECTED TRACE ELEMENTS

(Total concentrations in micrograms per liter, except as indicated)

$\begin{array}{lrlr}\text { Arsenic (As) } & 0 & \text { Manganese (Mn) } & 0 \\ \text { Barium (Ba) } & 0 & \text { Mercury (Hg) } & 0 \\ \text { Cadmium (Cd) } & 0 & \text { Selenium ( Se) } & 0 \\ \text { Chromium (Cr) } & 20 & \text { Silver (Ag) } & 0 \\ \text { Copper (Cu) } & 2 & \text { Strontium, dissolved (Sr) } & 190 \\ \text { Iron (Fe) } & 20 & \text { Zinc (Zn) } & 0 \\ \text { Lead (Pb) } & 7 & & \end{array}$

ANALYSIS OF SELECTED PESTICIDE AND INDUSTRIAL COMPOUNDS

(Total concentrations in micrograms per 1iter)

$\begin{array}{llll}\text { Aldrin } & .00 & \text { Lindane } & .00 \\ \text { Chlordane } & .0 & \text { Methoxych1or } & .00 \\ \text { DDD } & .00 & \text { Mirex } & .00 \\ \text { DDE } & .00 & \text { PCB } & .0 \\ \text { DDT } & .00 & \text { PCN } & .00 \\ \text { Dieldrin } & .00 & \text { Silvex } & .00 \\ \text { Endrin } & .00 & \text { Toxaphene } & 0 \\ \text { Heptach1or } & .00 & 2,4-D & .00 \\ \text { Heptachlor epoxide } & .00 & 2,4,5-\mathrm{T} & .00\end{array}$

ANALYSIS OF SELECTED GROSS RADIOACTIVITY

(Total activity concentrations in picocuries per liter)

Gross Alpha, as uranium natural $<2.2$

Gross Beta, as

cesium-137
Gross Beta, as

strontium -90 
PUBLIC WATER SUPPLY: North Miami

COUNTY: Dade

COLLECTION DATE: 11-30-77

SAMPLING POINT (1) Treated water-255352080084401, tap at house, 13195 Biscayne Drive.

(2) Raw water-

ANALYSIS OF MAJOR CHEMICAL CONSTITUENTS AND PHYSICAL PROPERTIES

(Dissolved concentrations in milligrams per liter, except as indicated)

(1) (2)

$\begin{array}{lc}\text { Silica ( } & 11 \\ \text { Calcium (Ca) } & 120 \\ \text { Magnesium (Mg) } & 24 \\ \text { Sodium (Na) } & 170 \\ \text { Potassium (K) } & 4.7 \\ \text { Bicarbonate (HCO3) } & 300 \\ \text { Carbonate (CO3) } & 0 \\ \text { Sulfate (SO4) } & 84 \\ \text { Chloride (C1) } & 310 \\ \text { F1uoride (F) } & .1 \\ \text { Nitrate (NO3-N), } & \\ \quad \text { total (NO2-N), } & .03 \\ \text { Nitrite tratal } & \\ \quad \text { total }\end{array}$

(1)

\section{ANALYSIS OF SELECTED TRACE ELEMENTS}

( Total concentrations in micrograms per liter, except as indicated)

$\begin{array}{lr}\text { Arsenic ( } \mathrm{As} \text { ) } & 0 \\ \text { Barium (Ba) } & -- \\ \text { Cadmium (Cd) } & 0 \\ \text { Chromium (Cr) } & 10 \\ \text { Copper (Cu) } & 3 \\ \text { Iron (Fe) } & 190 \\ \text { Lead (Pb) } & 21\end{array}$

Manganese (Mn)

Mercury ( $\mathrm{Hg}$ )

Selenium ( $\mathrm{Se}$ )

Silver (Ag)

Strontium, dissolved ( $\mathrm{Sr}$ ) 720

Zinc $(\mathrm{Zn})$
924

400

150

48

250

9.2

26.0

27

2

ANALYSIS OF SELECTED PESTICIDE AND INDUSTRIAL COMPOUNDS

( Total concentrations in micrograms per 1iter)

$\begin{array}{llll}\text { Aldrin } & .00 & \text { Lindane } & .00 \\ \text { Chlordane } & .0 & \text { Methoxychlor } & .00 \\ \text { DDD } & .00 & \text { Mirex } & .00 \\ \text { DDE } & .00 & \text { PCB } & .0 \\ \text { DDT } & .00 & \text { PCN } & .00 \\ \text { Dieldrin } & .00 & \text { Silvex } & .00 \\ \text { Endrin } & .00 & \text { Toxaphene } & 0 \\ \text { Heptachlor } & .00 & 2,4-D & .00 \\ \text { Heptachlor epoxide } & .00 & 2,4,5-T & .00\end{array}$

ANALYSIS OF SELECTED GROSS RADIOACTIVITY

(Total activity concentrations in picocuries per liter)

Gross Alpha, as uranium natura 1

Gross Beta, as

cesium-137
Gross Beta, as

strontium -90 
PUBLIC WATER SUPPLY: North Port Charlotte COUNTY: Sarasota COLLECTION DATE: 12-22-77

SAMPLING POINT (1) Treated water-270242082142409, water treatment plant laboratory tap.

(2) Raw water-270242082142401, Myakka-Hatchee River (Big Slough).

ANALYSIS OF MAJOR CHEMICAL CONSTITUENTS AND PHYSICAL PROPERTIES

(Dissolved concentrations in milligrams per liter, except as indicated)
(1)
(2)
(1)

(2)

Silica $\left(\mathrm{SiO}_{2}\right)$

Calcium $(\mathrm{Ca})$

Magnesium $(\mathrm{Mg})$

Sodium (Na)

Potassium (K)

Bicarbonate $\left(\mathrm{HCO}_{3}\right)$

Carbonate $\left(\mathrm{CO}_{3}\right)$

Sulfate $\left(\mathrm{SO}_{4}\right)$

Chloride (CL)

Fluoride (F)

Nitrate $\left(\mathrm{NO}_{3}^{-N}\right)$,

total

Nitrite $\left(\mathrm{NO}_{2}-\mathrm{N}\right)$,

total

\section{0}

43

12

$58 \quad 20$

$3.1 \quad 2.9$

$82 \quad 76$

$0 \quad 0$

$120 \quad 80$

$62 \quad 38$

$.2 \quad .3$

$.09 \quad .07$

$.00 \quad .02$
Dissolved solids

(residue at $180^{\circ} \mathrm{C}$ )

Hardness as $\mathrm{CaCO}_{3}$

$(\mathrm{Ca}, \mathrm{Mg}) \quad 160$

361

301

Noncarbonate hardness

as $\mathrm{CaCO}_{3}$

Percent sodium

Alkalinity as $\mathrm{CaCO}_{3}$

Specific conductance

(umhos $/ \mathrm{cm}$ at $25^{\circ} \mathrm{C}$ )

$\mathrm{pH}$ (units)

Temperature $\left({ }^{\circ} \mathrm{C}\right)$

Color (Pt-Co units)

Turbidity (NTU)
$160 \quad 160$

$91 \quad 100$

$44 \quad 21$

$67 \quad 62$

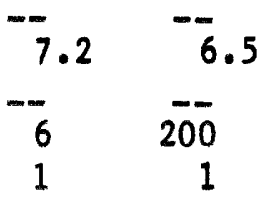

ANALYSIS OF SELECTED TRACE ELEMENTS

(Total concentrations in micrograms per liter, except as indicated)

Arsenic (As)

Barium ( $B a)$

Cadmium (Cd)

Chromium $(\mathrm{Cr})$

Copper ( $\mathrm{Cu})$

Iron ( $\mathrm{Fe})$

Lead $(\mathrm{Pb})$

$\begin{array}{rr}1 & 2 \\ -2 & - \\ 1 & 0 \\ 10 & <10 \\ 4 & 3 \\ 50 & 1000 \\ 3 & 0\end{array}$

Manganese (Mn)

Mercury ( $\mathrm{Hg}$ )

Selenium ( $\mathrm{Se}$ )

Silver ( $\mathrm{Ag}$ )

10

$<.5<.5$

Strontium, dissolved ( $\mathrm{Sr}$ ) 850

0

Zinc $(z n)$

ANALYSIS OF SELECTED PESTICIDE AND INDUSTRIAL COMPOUNDS

(Total concentrations in micrograms per liter)

$\begin{array}{llllll}\text { Aldrin } & .00 & .00 & \text { Lindane } & .00 & .00 \\ \text { Chlordane } & .0 & .0 & \text { Methoxychlor } & .00 & .00 \\ \text { DDD } & .00 & .00 & \text { Mirex } & .00 & .00 \\ \text { DDE } & .00 & .00 & \text { PCB } & .0 & .0 \\ \text { DDT } & .00 & .00 & \text { PCN } & .00 & .00 \\ \text { Dieldrin } & .00 & .00 & \text { Silvex } & .16 & .00 \\ \text { Endrin } & .00 & .00 & \text { Toxaphene } & 0 & 0 \\ \text { Heptachlor } & .00 & .00 & 2,4-D & .00 & .00 \\ \text { Heptachlor epoxide } & .00 & .00 & 2,4,5-T & .00 & .00\end{array}$

ANALYSIS OF SELECTED GRO \& RADIOACTIVITY

(Total activity concentrations in picocuries per liter)

Gross Alpha, as

uranium natural

Gross Beta, as

cesium-137
$5.7<3.2$

Gross Beta, as

strontium-90

$6.7 \quad 7.8$ 
PUBLIC WATER SUPPLY: North Port St. Lucie COUNTY: St. Lucie COLLECIION DATE: 11-30-77

SAMPLING POINT (1) Treated water-271828080203702, water treatment plant number 2, tap at City Hall.

(2) Raw water-

ANALYSIS OF MAJOR CHEMICAL CONSTITUENTS AND PHYSICAL PROPERTIES (Dissolved concentrations in milligrams per liter, except as indicated)

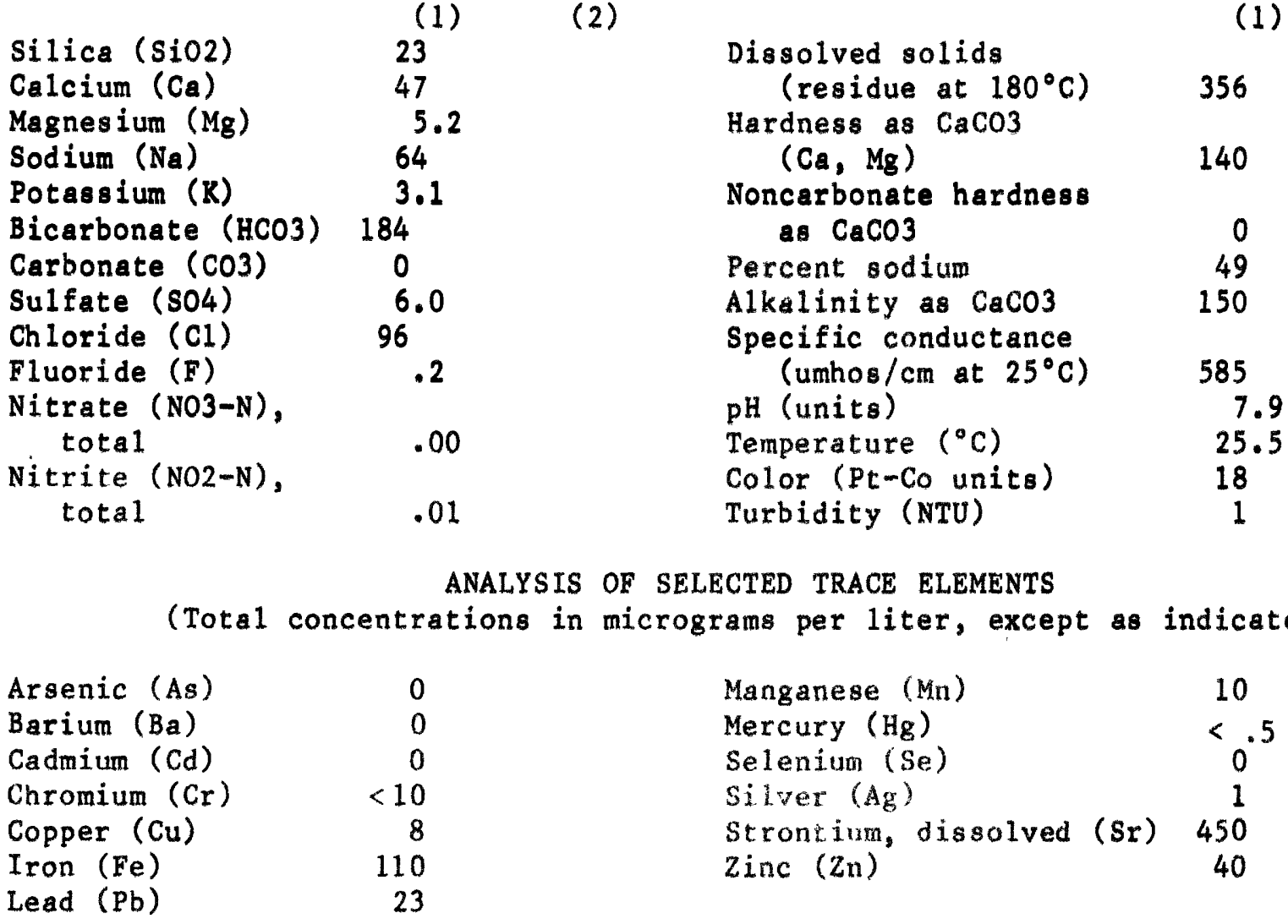

ANALYSIS OF SELECTED PESTICIDE AND INDUSTRIAL COMPOUNDS (Total concentrations in micrograms per liter)

$\begin{array}{llll}\text { Aldrin } & .00 & \text { Lindane } & .00 \\ \text { Chlordane } & .0 & \text { Methoxych1or } & .00 \\ \text { DDD } & .00 & \text { Mirex } & .00 \\ \text { DDE } & .00 & \text { PCB } & .0 \\ \text { DDT } & .00 & \text { PCN } & .00 \\ \text { Dieldrin } & .00 & \text { Silvex } & .02 \\ \text { Endrin } & .00 & \text { Toxaphene } & 0 \\ \text { Heptachlor } & .00 & 2,4-D & .02 \\ \text { Heptachlor epoxide } & .00 & 2,4,5-\mathrm{T} & .00\end{array}$

ANALYSIS OF SELECTED GROSS RADIOACTIVITY

(Total activity concentrations in picocuries per liter)

Gross Alpha, as uranium natural $<3.1$

Gross Beta, as

cesium-137
Gross Beta, as

strontium-90

2.3 
PUBLIC WATER SUPPLY: Okeechobee

COUNTY: Okeechobee

COLLECTION DATE: 11-10-77

SAMPLING POINT (1) Treated water-02276400, tap inside fence on high schoo1 grounds 1 mile north of Okeechobee.

(2) Raw water -02276400 , Lake Okeechobee intake at old water treatment plant.

ANALYSIS OF MAJOR CHEMICAL CONSTITUENTS AND PHYSICAL PROPERTIES

(Dissolved concentrations in milligrams per liter, except as indicated)

(1) (2)

Silica $\left(\mathrm{SiO}_{2}\right)$

Calcium ( $\mathrm{Ca})$

Magnesium $(\mathrm{Mg})$

Sodium ( $\mathrm{Na})$

Potassium (K)

Bicarbonate $\left(\mathrm{HCO}_{3}\right)$

Carbonate $\left(\mathrm{CO}_{3}\right)$

Sulfate $\left(\mathrm{SO}_{4}\right)$

Chloride (C1)

Fluoride (F)

Nitrate $\left(\mathrm{NO}_{3}-\mathrm{N}\right)$, total

Nitrite $\left(\mathrm{NO}_{2}-\mathrm{N}\right)$, total
11

\section{8}

$42 \quad 35$

$18 \quad 9.4$

$64 \quad 66$

$5.0 \quad 5.1$

$150 \quad 29$

$0 \quad 0$

$63 \quad 90$

$100 \quad 120$

$.3 \quad .2$

$.02 \quad .05$

$.01 \quad .00$
(1)

Dissolved solids

(residue at $180^{\circ} \mathrm{C}$ ) $\quad 417 \quad 405$

Hardness as $\mathrm{CaCO}_{3}$

$(\mathrm{Ca}, \mathrm{Mg}) \quad 180$

130

Noncarbonate hardness

as $\mathrm{CaCO}_{3}$

$57 \quad 100$

Percent sodium

Alkalinity as $\mathrm{CaCO}_{3}$

Specific conductance

(umhos $/ \mathrm{cm}$ at $25^{\circ} \mathrm{C}$ )

$\mathrm{pH}$ (units)

Temperature $\left({ }^{\circ} \mathrm{C}\right)$

Color (Pt-Co units)

Turbidity (NTU)

$43 \quad 52$

$120 \quad 24$

$\begin{array}{rr}- & - \\ 8.0 & 9.5 \\ 25.0 & 24.5 \\ 22 & 6 \\ 4 & 1\end{array}$

ANALYSIS OF SELECTED TRACE ELEMENTS

(Total concentrations in micrograms per liter, except as indicated)

Arsenic (As)

Barium ( $\mathrm{Ba}$ )

Cadmium ( $\mathrm{Cd}$ )

Chromium ( $\mathrm{Cr}$ )

Copper ( $\mathrm{Cu})$

Iron ( $\mathrm{Fe}$ )

Lead $(\mathrm{Pb})$

$\begin{array}{rr}1 & 2 \\ 0 & 0 \\ 0 & 1 \\ <10 & <10 \\ 1 & 6 \\ 100 & 160 \\ 0 & 2\end{array}$

Manganese $(\mathrm{Mn})$

Mercury ( $\mathrm{Hg}$ )

Selenium (Se)

Silver (Ag)

Strontium, dissolved (Sr) $1200 \quad 800$

Zinc $(\mathrm{Zn})$

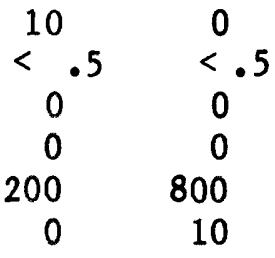

ANALYSIS OF SELECTED PESTICIDE AND INDUSTRIAL COMPOUNDS

(Total concentrations in micrograms per liter)

$\begin{array}{llllll}\text { Aldrin } & .00 & .00 & \text { Lindane } & .00 & .00 \\ \text { Chlordane } & .0 & .0 & \text { Methoxychlor } & .00 & .00 \\ \text { DDD } & .00 & .00 & \text { Mirex } & .00 & .00 \\ \text { DDE } & .00 & .00 & \text { PCB } & .0 & .0 \\ \text { DDT } & .00 & .00 & \text { PCN } & .00 & .00 \\ \text { Dieldrin } & .00 & .00 & \text { Silvex } & .00 & .00 \\ \text { Endrin } & .00 & .00 & \text { Toxaphene } & 0 & 0 \\ \text { Heptach1or } & .00 & .00 & 2,4-D & .00 & .00 \\ \text { Heptachlor epoxide } & .00 & .00 & 2,4,5-T & .00 & .00\end{array}$

ANALYSIS OF SELECTED GROSS RADIOACTIVITY

(Total activity concentrations in picocuries per 1iter)

Gross Alpha, as

uranium natural $<3.1<3.6$

Gross Beta, as

Cesium-137
8.19 .6
Gross Beta, as

strontium -90

7.2

8.6 
PUBLIC WATER SUPPLY: Orange Park

COUNTY: Clay

COLLECTION DATE: $12-14-77$

SAMPLING POINT (1) Treated water-301059081423901, tap 20 feet away from cylindrical holding rank at Ash Street water treatment plant.

(2) Raw water-

ANALYSIS OF MAJOR CHEMICAL CONSTITUENTS AND PHYSICAL PROPERTIES

(Dissolved concentrations in milligrams per liter, except as indicated)

$\begin{array}{lc}\text { Silica (SiO2) } & 16 \\ \text { Calcium (Ca) } & 34 \\ \text { Magnesium (Mg) } & 20 \\ \text { Sodium (Na) } & 6.8 \\ \text { Potassium (K) } & 2.5 \\ \text { Bicarbonate (HCO3) } & 120 \\ \text { Carbonate (CO3) } & 1 \\ \text { Sulfate (SO4) } & 71 \\ \text { Chloride (C1) } & 9.1 \\ \text { Fluoride (F) } & .6 \\ \text { Nitrate (NO3-N), } & \\ \quad \text { total (NO2-N), } & .01 \\ \text { Nitrite (NO2-N), } \\ \quad \text { total }\end{array}$

(2)

(1)

Dissolved solids

(residue at $180^{\circ} \mathrm{C}$ ) 213

Hardness as $\mathrm{CaCO} 3$

$(\mathrm{Ca}, \mathrm{Mg}$ )

170

Noncarbonate hardness

as $\mathrm{CaCO} 3$

Percent sodium

70

Alkalinity as $\mathrm{CaCO} 3 \quad 100$

Specific conductance

(umhos $/ \mathrm{cm}$ at $25^{\circ} \mathrm{C}$ )

$\mathrm{pH}$ (units)

Temperature $\left({ }^{\circ} \mathrm{C}\right)$

Color (Pt-Co units)

Turbidity (NTU)

100

380

$--$

23.5

0

1

ANALYSIS OF SELECTED TRACE ELEMENTS

(Total concentrations in micrograms per liter, except as indicated)

$\begin{array}{lrlr}\text { Arsenic (As) } & 1 & \text { Manganese (Mn) } & 0 \\ \text { Barium (Ba) } & -- & \text { Mercury (Hg) } & <.5 \\ \text { Cadmium (Cd) } & 0 & \text { Selenium (Se) } & 0 \\ \text { Chromium (Cr) } & <10 & \text { Silver (Ag) } & 1 \\ \text { Copper (Cu) } & 13 & \text { Strontium, dissolved (Sr) } 2900 \\ \text { Iron (Fe) } & 30 & \text { Zinc (Zn) } & 10 \\ \text { Lead (Pb) } & 5 & & \end{array}$

ANALYSIS OF SELECTED PESTICIDE AND INDUSTRIAL COMPOUNDS

(Total concentrations in micrograms per liter)

$\begin{array}{llll}\text { Aldrin } & .00 & \text { Lindane } & .00 \\ \text { Chlordane } & .0 & \text { Methoxychlor } & .00 \\ \text { DDD } & .00 & \text { Mirex } & .00 \\ \text { DDE } & .00 & \text { PCB } & .0 \\ \text { DDT } & .00 & \text { PCN } & .00 \\ \text { Dieldrin } & .00 & \text { Silvex } & .00 \\ \text { Endrin } & .00 & \text { Toxaphene } & 0 \\ \text { Heptachlor } & .00 & 2,4-D & .00 \\ \text { Heptachlor epoxide } & .00 & 2,4,5-T & .00\end{array}$

ANALYSIS OF SELECTED GROSS RADIOACTIVITY

(Total activity concentrations in picocuries per liter)

$\begin{array}{ll}\text { Gross Alpha, as } & \\ \text { uranium natural } & <2.1 \\ \begin{array}{c}\text { Gross Beta, as } \\ \text { cesium-137 }\end{array} & 2.7\end{array}$

Gross Beta, as

strontium-90 2.4 
PUBLIC WATER SUPPLY: Pace

COUNTY: Santa Rosa

COLLECTION DATE: 01-05-78

SAMPLING POINT (1) Treated water-303630087084090, tap at west end of Spruce

Street baseball diamond near shopping center.

(2) Raw water-

ANALYSIS OF MAJOR CHEMICAL CONSTITUENTS AND PHYSICAL PROPERTIES

(Dissolved concentrations in milligrams per liter, except as indicated)

(1) (2)

Silica (SiO2)

Calcium ( $\mathrm{Ca}$ )

Magnesium ( $\mathrm{Mg}$ )

Sodium ( $\mathrm{Na})$

Potassium (K)

Bicarbonate (HCO3)

Carbonate ( $\mathrm{CO} 3$ )

Sulfate (S04)

Chloride (C1)

Fluoride (F)

Nitrate (NO3-N), total

Nitrite (NO2-N), total
7.0

7.6

.5

2.1

.2

23

0

.7

4.2

.0

.34

.00
Dissolved solids

(residue at $180^{\circ} \mathrm{C}$ )

(1)

42

Hardness as $\mathrm{CaCO} 3$

( $\mathrm{C} a, \mathrm{Mg}$ )

21

Noncarbonate hardness as $\mathrm{CaCO} 3$

Percent sodium

Alkalinity as $\mathrm{CaCO} 3$

Specific conductance

(umhos $/ \mathrm{cm}$ at $25^{\circ} \mathrm{C}$ )

$\mathrm{pH}$ (units)

Temperature $\left({ }^{\circ} \mathrm{C}\right)$

Color (Pt-Co units)

Turbidity (NTU)

ANALYSIS OF SELECTED TRACE ELEMENTS

(Total concentrations in micrograms per liter, except as indicated)

$\begin{array}{lr}\text { Arsenic ( } \mathrm{As} \text { ) } & 0 \\ \text { Barium (Ba) } & -- \\ \text { Cadmium ( } \mathrm{Cd} \text { ) } & 1 \\ \text { Chromium ( } \mathrm{Cr} \text { ) } & <10 \\ \text { Copper (Cu) } & 9 \\ \text { Iron (Fe) } & 60 \\ \text { Lead (Pb) } & 80\end{array}$

Manganese (Mn)

Mercury ( $\mathrm{Hg}$ )

Selenium ( $\mathrm{Se}$ )

Silver ( $\mathrm{Ag}$ )

Strontium, dissolved ( $\mathrm{Sr}$ ) 40

Zinc $(2 n)$
0

$<.5$

0

0

ANALYSIS OF SELECTED PESTICIDE AND INDUSTRIAL COMPOUNDS

(Total concentrations in micrograms per liter)

$\begin{array}{llll}\text { Aldrin } & .00 & \text { Lindane } & .00 \\ \text { Chlordane } & .0 & \text { Methoxychlor } & .00 \\ \text { DDD } & .00 & \text { Mirex } & .00 \\ \text { DDE } & .00 & \text { PCB } & .0 \\ \text { DDT } & .00 & \text { PCN } & .00 \\ \text { Dieldrin } & .00 & \text { Silvex } & .00 \\ \text { Endrin } & .00 & \text { Toxaphene } & 0 \\ \text { Heptachlor } & .00 & 2,4-D & .00 \\ \text { Heptachlor epoxide } & .00 & 2,4,5-T & .00\end{array}$

ANALYSIS OF SELECTED GROSS RADIOACTIVITY

(Total activity concentrations in picocuries per liter)

Gross Alpha, as uranium natural $\quad .68$

Gross Beta, as

cesium-137
Gross Beta, as

strontium -90 
PUBLIC WATER SUPPLY: Pahokee

COUNTY: Palm Beach

COLLECTION DATE: 12-06-77

SAMPLING POINT (1) Treated water-264930080400502, water treatment plant laboratory tap.

(2) Raw water-264930080400501, water treatment plant laboratory tap.

ANALYSIS OF MAJOR CHEMICAL CONSTITUENTS AND PHYSICAL PROPERTIES (Dissolved concentrations in milligrams per liter, except as indicated)

(1) (2)

Silica $\left(\mathrm{SiO}_{2}\right)$
Calcium $(\mathrm{Ca})$
Magnesium $(\mathrm{Mg})$
Sodium ( $\mathrm{Na})$
Potassium (K)
Bicarbonate $\left(\mathrm{HCO}_{3}\right.$
Carbonate $\left(\mathrm{CO}_{3}\right)$
Sulfate $\left(\mathrm{SO}_{4}\right)$
Chloride $(\mathrm{Cl})$
Fluoride $(\mathrm{F})$
Nitrate $\left(\mathrm{NO}_{3}-\mathrm{N}\right)$,
total
Nitrite $\left(\mathrm{NO}_{2}-\mathrm{N}\right)$,
total

12

$44-51$

$13 \quad 22$

$66 \quad 66$

$5.1 \quad 5.2$

$60 \quad 176$

$8 \quad 0$

$85 \quad 70$

$120 \quad 100$

$.4 \quad .4$

$.29 \quad .25$

$.00 \quad .01$
Dissolved solids

(1)

402

Hardness as $\mathrm{CaCO}_{3}$

$$
(\mathrm{Ca}, \mathrm{Mg})
$$

160

220

Noncarbonate hardness as $\mathrm{CaCO}_{3}$

100

76

Percent sodium

Alkalinity as $\mathrm{CaCO}_{3}$

Specific conductance

(umhos $/ \mathrm{cm}$ at $25^{\circ} \mathrm{C}$ )

$\mathrm{pH}$ (units)

Temperature $\left({ }^{\circ} \mathrm{C}\right)$

Color (Pt-Co units)

Turbidity (NTU)

$46 \quad 39$

$63 \quad 144$

$640 \quad 690$

$9.3 \quad 8.1$

$25.5 \quad 24.0$

$11 \quad 70$

11

ANALYSIS OF SELECTED TRACE ELEMENTS

(Total concentrations in micrograms per liter, except as indicated)

$\begin{array}{lrrlrr}\text { Arsenic (As) } & 0 & 2 & \text { Manganese (Mn) } & 10 & 10 \\ \text { Barium (Ba) } & 0 & 0 & \text { Mercury (Hg) } & <.5 & <.5 \\ \text { Cadmium (Cd) } & 1 & 1 & \text { Selenium (Se) } & 0 & 0 \\ \text { Chromium (Cr) } & 10 & <10 & \text { Silver (Ag) } & 0 \\ \text { Copper (Cu) } & 3 & 8 & \text { Strontium, dissolved (Sr) } & 810 & 1200 \\ \text { Iron (Fe) } & 50 & 680 & \text { Zinc (Zn) } & 10 & 100 \\ \text { Lead (Pb) } & 20 & 5 & & \end{array}$

ANALYSIS OF SELECTED PESTICIDE AND INDUSTRIAL COMPOUNDS

(Total concentrations in micrograms per liter)

$\begin{array}{llllll}\text { Aldrin } & .00 & .00 & \text { Lindane } & .00 & .00 \\ \text { Chlordane } & .0 & .0 & \text { Methoxych1or } & .00 & .00 \\ \text { DDD } & .00 & .00 & \text { Mirex } & .00 & .00 \\ \text { DDE } & .00 & .00 & \text { PCB } & .0 & .0 \\ \text { DDT } & .00 & .00 & \text { PCN } & .00 & .00 \\ \text { Dieldrin } & .00 & .00 & \text { Silvex } & .00 & .00 \\ \text { Endrin } & .00 & .00 & \text { Toxaphene } & 0 & 0 \\ \text { Heptachlor } & .00 & .00 & 2,4-D & .00 & .00 \\ \text { Heptachlor epoxide } & .00 & .00 & 2,4,5-T & .00 & .00\end{array}$

ANALYSIS OF SELECTED GROSS RADIOACTIVITY

(Total activity concentrations in picocuries per liter)

Gross Alpha, as

uranium natural $\quad<4.9$

Gross Beta, as

cesium-137
Gross Beta, as

$5.8 \quad$ strontium-90

9.610 
PUBLIC WATER SUPPLY: Palm Beach-Villa Del Ray COUNTY: Palm Beach COLLECTION DATE: 11-25-77

SAMPLING POINT (1) Treated water-262857080084202, Palm Beach County Utilities at Clubhouse Administration building near maingate Kings Point, tap at SE corner.

(2) Raw water-

ANALYSIS OF MAJOR CHEMICAL CONSTITUENTS AND PHYSICAL PROPERTIES (Dissolved concentrations in milligrams per liter, except as indicated)

(1) (2)

18

52

2.0

20

1.8

144

0

2.0

45

.2

.03

.01
(1)

Dissolved solids

(residue at $180^{\circ} \mathrm{C}$ ) 249

Hardness as $\mathrm{CaCO} 3$

$(\mathrm{Ca}, \mathrm{Mg})$

140

Noncarbonate hardness as $\mathrm{CaCO} 3$

20

Percent sodium 24

Alkalinity as $\mathrm{CaCO} 3 \quad 120$

Specific conductance

(umhos $/ \mathrm{cm}$ at $25^{\circ} \mathrm{C}$ )

pH (units)

Temperature $\left({ }^{\circ} \mathrm{C}\right)$

Color (Pt-Co units)

Turbidity (NTU)

ANALYSIS OF SELECTED TRACE ELEMENTS

(Total concentrations in micrograms per liter, except as indicated)

$\begin{array}{lr}\text { Arsenic (As) } & 0 \\ \text { Barium (Ba) r } & 0 \\ \text { Cadmium (Cd) } & 0 \\ \text { Chromium (Cr) } & <10 \\ \text { Copper ( Cu) } & 130 \\ \text { Iron (Fe) } & 30 \\ \text { Lead (Pb) } & 28\end{array}$

$\begin{array}{lc}\text { Manganese (Mn) } & 0 \\ \text { Mercury (Hg) } & <\cdot 5 \\ \text { Selenium (Se) } & 0 \\ \text { Silver (Ag) } & 0 \\ \text { Strontium, dissolved ( } \mathrm{Sr}) & 370 \\ \text { Zinc (Zn) } & 0\end{array}$

ANALYSIS OF SELECTED PESTICIDE AND INDUSTRIAL COMPOUNDS

(Total concentrations in micrograms per liter)

Aldrin

Chlordane

DDD

DDE

DDT

Dieldrin

Endrin

Heptach 1 or

Heptachlor epoxide

$\begin{array}{ll}.00 & \text { Lindane } \\ .0 & \text { Methoxychlor } \\ .00 & \text { Mirex } \\ .00 & \text { PCB } \\ .00 & \text { PCN } \\ .00 & \text { Silvex } \\ .00 & \text { Toxaphene } \\ .00 & 2,4-D \\ .00 & 2,4,5-\mathrm{T}\end{array}$

.00

.00

.00

.0

.00

.00

0

.02

.00

ANALYSIS OF SELECTED GROSS RADIOACTIVITY

(Total activity concentrations in picocuries per liter)

Gross Alpha, as uranium natural Gross Beta, as cesium-137
Gross Beta, as

strontium-90 
PUBLIC WATER SUPPLY: Palm Coast

COUNTY: Flagler

COLLECTION DATE: 11-09-77

SAMPLING POINT (1) Treated water-293324081130701, tap in back of water treatment plant.

(2) Raw water-

ANALYSIS OF MAJOR CHEMICAL CONSTITUENTS AND PHYSICAL PROPERTIES

(Dissolved concentrations in milligrams per liter, except as indicated)

$\begin{array}{lc}\text { Silica (SiO2) } & 27 \\ \text { Calcium (Ca) } & 28 \\ \text { Magnesium (Mg) } & 3.4 \\ \text { Sodium (Na) } & 26 \\ \text { Potassium (K) } & 1.1 \\ \text { Bicarbonate (HCO3) } & 89 \\ \text { Carbonate (CO3) } & 0 \\ \text { Sulfate (SO4) } & 1.0 \\ \text { Chloride (C1) } & 50 \\ \text { Fluoride (F) } & .1 \\ \text { Nitrate (NO3-N), } & \\ \quad \text { total (NO2-N), } & .02 \\ \text { Nitrite } & \\ \quad \text { total }\end{array}$

(2)

(1)

Dissolved solids

(residue at $180^{\circ} \mathrm{C}$ ) $\quad 214$

Hardness as $\mathrm{CaCO} 3$

( $\mathrm{Ca}, \mathrm{Mg}) \quad 84$

Noncarbonate hardness

as $\mathrm{CaCO} 3$

11

Percent sodium $\quad 40$

Alkalinity as $\mathrm{CaCO} 3 \quad 73$

Specific conductance

(umhos $/ \mathrm{cm}$ at $25^{\circ} \mathrm{C}$ )

$\mathrm{pH}$ (units)

313

Temperature $\left({ }^{\circ} \mathrm{C}\right)$

Color (Pt-Co units)

7.8

Turbidity (NTU)

ANALYSIS OF SELECTED TRACE ELEMENTS

( Total concentrations in micrograms per liter, except as indicated)

$\begin{array}{lrlr}\text { Arsenic (As) } & 0 & \text { Manganese (Mn) } & 0 \\ \text { Barium (Ba) } & 0 & \text { Mercury (Hg) } & <.5 \\ \text { Cadmium (Cd) } & 0 & \text { Selenium (Se) } & 0 \\ \text { Chromium (Cr) } & 10 & \text { Silver (Ag) } & 1 \\ \text { Copper (Cu) } & 20 & \text { Strontium, dissolved (Sr) } & 300 \\ \text { Iron (Fe) } & 40 & \text { Zinc (Zn) } & 0 \\ \text { Lead (Pb) } & 1 & & \end{array}$

ANALYSIS OF SELECTED PESTICIDE AND INDUSTRIAL COMPOUNDS

(Total concentrations in micrograms per 1iter)

$\begin{array}{llll}\text { Aldrin } & .00 & \text { Lindane } & .00 \\ \text { Chlordane } & .0 & \text { Methoxychlor } & .00 \\ \text { DDD } & .00 & \text { Mirex } & .0 \\ \text { DDE } & .00 & \text { PCB } & .00 \\ \text { DDT } & .00 & \text { PCN } & .00 \\ \text { Dieldrin } & .00 & \text { Silvex } & 0 \\ \text { Endrin } & .00 & \text { Toxaphene } & .00 \\ \text { Heptachlor } & .00 & 2,4-D & .00 \\ \text { Heptachlor epoxide } & .00 & 2,4,5-\mathrm{T} & \end{array}$

ANALYSIS OF SELECTED GROSS RADIOACTIVITY

(Total activity concentrations in picocuries per liter)

Gross Alpha, as

uranium natural $<2.0$

Gross Beta, as

cesium-137
Gross Beta, as

strontium-90

1.4 
PUBLIC WATER SUPPLY: Panacea

COUNTY: Waku1la

COLLECTION DATE: 11-30-77

SAMPLING POINT (1) Treated water-300151084235890, tap on southwest corner of gasoline station at Highway 98 and A-372.

(2) Raw water-

ANALYSIS OF MAJOR CHEMICAL CONSTITUENTS AND PHYSICAL PROPERTIES

(Dissolved concentrations in milligrams per liter, except as indicated)

$\begin{array}{lc}\text { Silica ( } & \text { SiO2) } \\ \text { Calcium (Ca) } & 30 \\ \text { Magnesium (Mg) } & 1.6 \\ \text { Sodium (Na) } & 7.8 \\ \text { Potassium (K) } & 1.0 \\ \text { Bicarbonate (HCO3) } & 94 \\ \text { Carbonate (CO3) } & 0 \\ \text { Sulfate (SO4) } & 2.2 \\ \text { Chloride (C1) } & 10 \\ \text { Fluoride (F) } & .0 \\ \text { Nitrate (NO3-N), } & \\ \quad \text { tota1 (NO2-N), } & .38 \\ \text { Nitrite (Notal } & \\ \quad \text { total }\end{array}$

(2)

(1)

Dissolved solids

(residue at $180^{\circ} \mathrm{C}$ ) $\quad 100$

Hardness as $\mathrm{CaCO} 3$

( $\mathrm{Ca}, \mathrm{Mg}) \quad 82$

Noncarbonate hardness

as $\mathrm{CaCO} 3$

Percent sodium $\quad 17$

Alkalinity as $\mathrm{CaCO} 3 \quad 77$

Specific conductance

(umhos $/ \mathrm{cm}$ at $25^{\circ} \mathrm{C}$ )

197

pH (units)

Temperature $\left({ }^{\circ} \mathrm{C}\right)$

7.6

Color (Pt-Co units)

21.5

.00

Turbidity (NTU)

2

0

ANALYSIS OF SELECTED TRACE ELEMENTS

(Total concentrations in micrograms per liter, except as indicated)

\begin{tabular}{|c|c|c|c|}
\hline Arsenic (As) & 0 & Manganese $(\mathrm{Mn})$ & 0 \\
\hline Barium ( $\mathrm{Ba})$ & 0 & Mercury (Hg) & $<.5$ \\
\hline Cadmium (Cd) & 2 & Selenium ( $\mathrm{Se}$ ) & 0 \\
\hline Chromium (Cr) & 40 & Silver $(\mathrm{Ag})$ & 0 \\
\hline Copper $(\mathrm{Cu})$ & 6 & Strontium, dissolved ( $\mathrm{Sr}$ ) & 60 \\
\hline Iron (Fe) & 30 & Zinc $(\mathrm{Zn})$ & 80 \\
\hline Lead (Pb) & 3 & & \\
\hline
\end{tabular}

ANALYSIS OF SELECTED PESTICIDE AND INDUSTRIAL COMPOUNDS

(Total concentrations in micrograms per liter)

$\begin{array}{llll}\text { Aldrin } & .00 & \text { Lindane } & .00 \\ \text { Chlordane } & .0 & \text { Methoxychlor } & .00 \\ \text { DDD } & .00 & \text { Mirex } & .00 \\ \text { DDE } & .00 & \text { PCB } & .0 \\ \text { DDT } & .00 & \text { PCN } & .00 \\ \text { Dieldrin } & .00 & \text { Silvex } & .00 \\ \text { Endrin } & .00 & \text { Toxaphene } & 0 \\ \text { Heptachlor } & .00 & 2,4-\mathrm{D} & .00 \\ \text { Heptachlor epoxide } & .00 & 2,4,5-\mathrm{T} & .00\end{array}$

ANALYSIS OF SELECTED GROSS RADIOACTIVITY

(Total activity concentrations in picocuries per liter)

Gross Alpha, as

uranium natural --

Gross Beta, as

cesium-137
Gross Beta, as

strontium-90 
PUBLIC WATER SUPPLY: Paxton

COUNTY: Walton

COLLECTION DATE: 01-04-78

SAMPLING POINT (1) Treated water-305828086180990, tap on northwest corner of City Hall/fire station on Highway 331 .

(2) Raw water-

ANALYSIS OF MAJOR CHEMICAL CONSTITUENTS AND PHYSICAL PROPERTIES (Dissolved concentrations in milligrams per liter, except as indicated)

(1) (2)

$\begin{array}{lc}\text { Silica (SiO2) } & 11 \\ \text { Calcium (Ca) } & 23 \\ \text { Magnesium (Mg) } & 13 \\ \text { Sodium (Na) } & 2.4 \\ \text { Potassium (K) } & .9 \\ \text { Bicarbonate (HCO3) } & 120 \\ \text { Carbonate (CO3) } & 0 \\ \text { Sulfate (SO4) } & 7.8 \\ \text { Chloride (C1) } & 2.5 \\ \text { Fluoride (F) } & .1 \\ \text { Nitrate (NO3-N), } & \\ \quad \text { total (NO2-N), } & .01 \\ \text { Nitrite (NO2-N) } & .00 \\ \quad \text { total } & \end{array}$

Dissolved solids

(1)

$$
\text { (residue at } 180^{\circ} \mathrm{C} \text { ) } \quad 114
$$

Hardness as $\mathrm{CaCO} 3$ $(\mathrm{Ca}, \mathrm{Mg}) \quad 110$

Noncarbonate hardness as $\mathrm{CaCO} 3$

Percent sodium

Alkalinity as $\mathrm{CaCO} 3$

Specific conductance

(umhos $/ \mathrm{cm}$ at $25^{\circ} \mathrm{C}$ )

$\mathrm{pH}$ (units)

Temperature $\left({ }^{\circ} \mathrm{C}\right)$

Color (Pt-Co units)

Turbidity (NTU)

13
4
98
190
7.8
11.0
2
0

ANALYSIS OF SELECTED TRACE ELEMENTS

(Total concentrations in micrograms per liter, except as indicated)

$\begin{array}{lr}\text { Arsenic (As) } & 1 \\ \text { Barium (Ba) } & - \\ \text { Cadmium (Cd) } & 0 \\ \text { Chromium (Cr) } & <10 \\ \text { Copper (Cu) } & 6 \\ \text { Iron (Fe) } & 0 \\ \text { Lead (Pb) } & 16\end{array}$

$\begin{array}{lc}\text { Manganese (Mn) } & 0 \\ \text { Mercury (Hg) } & <.5 \\ \text { Selenium (Se) } & 0 \\ \text { Silver (Ag) } & 0 \\ \text { Strontium, dissolved (Sr) } & 150 \\ \text { Zinc ( } \mathrm{Zn} \text { ) } & 50\end{array}$

ANALYSIS OF SELECTED PESTICIDE AND INDUSTRIAL COMPOUNDS

(Total concentrations in micrograms per liter)

$\begin{array}{llll}\text { Aldrin } & .00 & \text { Lindane } & .00 \\ \text { Chlordane } & .0 & \text { Methoxychlor } & .00 \\ \text { DDD } & .00 & \text { Mirex } & .00 \\ \text { DDE } & .00 & \text { PCB } & .0 \\ \text { DDT } & .00 & \text { PCN } & .00 \\ \text { Dieldrin } & .00 & \text { Silvex } & .00 \\ \text { Endrin } & .00 & \text { Toxaphene } & 0 \\ \text { Heptachlor } & .00 & 2,4-D & .00 \\ \text { Heptachlor epoxide } & .00 & 2,4,5-\mathrm{T} & .00\end{array}$

ANALYSIS OF SELECTED GROSS RADIOACTIVITY

(Total activity concentrations in picocuries per liter)

Gross Alpha, as

uranium natural $<1.4$

Gross Beta, as

cesium-137
Gross Beta, as

strontium -90 
PUBLIC WATER SUPPLY: Pembroke Pines

COUNTY: Broward COLLECTION DATE: 11-22-77

SAMPLING POINT (1) Treated water-260114080183001, tap at house, 110th Avenue and Taft Street.

(2) Raw water-

ANALYSIS OF MAJOR CHEMICAL CONSTITUENTS AND PHYSICAL PROPERTIES

(Dissolved concentrations in milligrams per liter, except as indicated)

Silica (SiO2)

Calcium ( $\mathrm{Ca}$ )

Magnesium ( $\mathrm{Mg})$

Sodium ( $\mathrm{Na})$

Potassium ( $\mathrm{K}$ )

Bicarbonate ( $\mathrm{HCO} 3)$

Carbonate ( $\mathrm{CO} 3$ )

Sulfate (S04)

Chloride (C1)

Fluoride (F)

Nitrate $(\mathrm{NO3}-\mathrm{N})$,

total

Nitrite (NO2-N), total
(1)

9.6

(2)

\section{2}

4.2

13

1.4

19

0

16

60

.1

.06

.00
(1)

Dissolved solids

(residue at $180^{\circ} \mathrm{C}$ )

153

Hardness as $\mathrm{CaCp} 3$

$(\mathrm{Ca}, \mathrm{Mg}$ )

73

Noncarbonate hardness as $\mathrm{CaCO} 3$

\section{7}

Percent sodium 28

Alkalinity as $\mathrm{CaCO} 3 \quad 16$

Specific conductance

(umhos $/ \mathrm{cm}$ at $25^{\circ} \mathrm{C}$ )

230

$\mathrm{pH}$ (units)

7.5

Temperature $\left({ }^{\circ} \mathrm{C}\right) \quad 25.0$

Color (Pt-Co units) 11

Turbidity (NTU)

ANALYSIS OF SELECTED TRACE ELEMENTS

(Total concentrations in micrograms per liter, except as indicated)

Arsenic (As)

Barium ( $\mathrm{Ba}$ )

Cadmium ( $\mathrm{Cd}$ )

Chromium ( $\mathrm{Cr}$ )

Copper ( $\mathrm{Cu})$

Iron ( $\mathrm{Fe}$ )

Lead $(\mathrm{Pb})$

\begin{tabular}{r|}
1 \\
-1 \\
$<10$ \\
62 \\
150 \\
32
\end{tabular}

Manganese (Mn)

Mercury ( $\mathrm{Hg})$

Selenium ( $\mathrm{Se}$ )

Silver (Ag)

Strontium, dissolved ( $\mathrm{Sr}$ ) 250

Zinc $(\mathrm{Zn})$
10

.6

0

360

ANALYSIS OF SELECTED PESTICIDE AND INDUSTRIAL COMPOUNDS

(Total concentrations in micrograms per liter)

$\begin{array}{llll}\text { Aldrin } & .00 & \text { Lindane } & .00 \\ \text { Chlordane } & .0 & \text { Methoxychlor } & .00 \\ \text { DDD } & .00 & \text { Mirex } & .00 \\ \text { DDE } & .00 & \text { PCB } & .0 \\ \text { DDT } & .00 & \text { PCN } & .00 \\ \text { Dieldrin } & .00 & \text { Silvex } & .00 \\ \text { Endrin } & .00 & \text { Toxaphene } & 0 \\ \text { Heptachlor } & .00 & 2,4-D & .00 \\ \text { Heptachlor epoxide } & .00 & 2,4,5-T & .00\end{array}$

ANALYSIS OF SELECTED GROSS RADIOACTIVITY

(Total activity concentrations in picocuries per liter)

Gross Alpha, as

uranium natural

Gross Beta, as

cesium-137
2.3

Gross Beta, as

strontium-90

1.2 
PUBLIC WATER SUPPLY: Port Charlotte

COUNTY: Charlotte

COLLECTION DATE: 12-22-77

SAMPLING POINT (1) Treated water-265924082044709, Port Charlotte water water treatment plant No. 2, tap at sink in laboratory (from Fordham Waterway). (2) Raw water-265924082044701, taken from canal along side the water treatment plant.

ANALYSIS OF MAJOR CHEMICAL CONSTITUENTS AND PHYSICAL PROPERTIES (Dissolved concentrations in milligrams per liter, except as indicated)
(1)
(2)
(1)

$6.8 \quad 8.2$

Silica (SiO2)

$31 \quad 91$

Calcium ( $\mathrm{Ca}$ )

Magnesium ( $\mathrm{Mg}$ )

Sodium (Na)

$\begin{array}{ll}6.7 & 7.3\end{array}$

Potassium (K)

$74 \quad 33$

Bicarbonate ( $\mathrm{HCO3}$ )

2.3

Carbonate ( $\mathrm{CO} 3)$

Sulfate (S04)

100

33

0

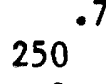

.7

0

$83 \quad 45$

Chloride (C1)

77

45

Fluoride (F)

\begin{tabular}{ll}
$77 \quad 58$ \\
\hline
\end{tabular}

$.2 \quad .3$

Nitrate (NO3-N), total

$.02 \quad .01$

Nitrite (NO2-N), total

$.00 \quad .00$

Dissolved solids

.00 Turbidity (NTU)

Hardness as $\mathrm{CaCO} 3$

$(\mathrm{Ca}, \mathrm{Mg})$

346

110

398

Noncarbonate hardness as $\mathrm{CaCO} 3$

23

60

Percent sodium

Alkalinity as $\mathrm{CaCO} 3$

Specific conductance

(umhos $/ \mathrm{cm}$ at $25^{\circ} \mathrm{C}$ )

pH (units)

Temperature $\left({ }^{\circ} \mathrm{C}\right)$

Color (Pt-Co units)

$82 \quad 210$

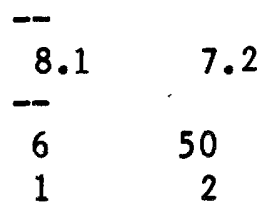

ANALYSIS OF SELECTED TRACE ELEMENTS

(Total concentrations in micrograms per liter, except as indicated)

$\begin{array}{lrr}\text { Arsenic (As) } & -- & 1 \\ \text { Barium (Ba) } & -- & - \\ \text { Cadmium (Cd) } & 0 & 0 \\ \text { Chromium (Cr) } & 10 & 10 \\ \text { Copper (Cu) } & 4 & 20 \\ \text { Iron (Fe) } & 70 & 300 \\ \text { Lead (Pb) } & 0 & 2\end{array}$

$\begin{array}{lrc}\text { Manganese (Mn) } & 10 & 10 \\ \text { Mercury (Hg) } & -- & <.5 \\ \text { Selenium (Se) } & -- & 0 \\ \text { Silver (Ag) } & 0 & 0 \\ \text { Strontium, dissolved (Sr) } & 390 & 780 \\ \text { Zinc ( } \mathrm{n} \text { ) } & 10 & 10\end{array}$

ANALYSIS OF SELECTED PESTICIDE AND INDUSTRIAL COMPOUNDS

(Total concentrations in micrograms per liter)

$\begin{array}{llllll}\text { Aldrin } & .00 & .00 & \text { Lindane } & .00 & .00 \\ \text { Chlordane } & .0 & .0 & \text { Methoxychlor } & .00 & .00 \\ \text { DDD } & .00 & .00 & \text { Mirex } & .00 & .00 \\ \text { DDE } & .00 & .00 & \text { PCB } & .0 & .0 \\ \text { DDT } & .00 & .00 & \text { PCN } & .00 & .00 \\ \text { Dieldrin } & .00 & .00 & \text { Silvex } & .00 & - \\ \text { Endrin } & .00 & .00 & \text { Toxaphene } & 0 & 0 \\ \text { Heptachlor } & .00 & .00 & 2,4-D & .00 & -- \\ \text { Heptachlor epoxide } & .00 & .00 & 2,4,5-T & .00 & -\end{array}$

ANALYSIS OF SELECTED GROSS RADIOACTIVITY

(Total activity concentrations in picocuries per liter)

Gross Alpha, as uranium natural

Gross Beta, as

cesium-137
$5.2--$

1.9
Gross Beta, as

strontium -90
$1.7 \quad-$ 
PUBLIC WATER SUPPLY: Port Richey COUNTY: Pasco COLLECTION DATE: $12-19-77$

SAMPLING POINT (1) Treated water-281648082424809, tap outside water treatment plant on south side.

(2) Raw water-

ANALYSIS OF MAJOR CHEMICAL CONSTITUENTS AND PHYSICAL PROPERTIES (Dissolved concentrations in milligrams per liter, except as indicated)

(1) (2)

$\begin{array}{lc}\text { Silica (Si02) } & 11 \\ \text { Calcium (Ca) } & 69 \\ \text { Magnesium (Mg) } & 7.0 \\ \text { Sodium (Na) } & 58 \\ \text { Potassium (K) } & 2.2 \\ \text { Bicarbonate (HCO3) } & 170 \\ \text { Carbonate (CO3) } & 0 \\ \text { Sulfate (SO4) } & 11 \\ \text { Chloride (C1) } & 120 \\ \text { Fluoride (F) } & .1 \\ \text { Nitrate (NO3-N), } & \\ \quad \text { total (NO2-N), } & .01 \\ \text { Nitrite (NO2-N) } & \\ \quad \text { total } & .00\end{array}$

Dissolved solids

(residue at $180^{\circ} \mathrm{C}$ ) $\quad 401$ Hardness as $\mathrm{CaCO} 3$ $(\mathrm{Ca}, \mathrm{Mg}$ )

(1)

200

Noncarbonate hardness as $\mathrm{CaCO} 3$

62

Percent sodium 38

Alkalinity as $\mathrm{CaCO} 3 \quad 140$

Specific conductance

(umhos $/ \mathrm{cm}$ at $25^{\circ} \mathrm{C}$ )

$\mathrm{pH}$ (units)

Temperature $\left({ }^{\circ} \mathrm{C}\right)$

Color (Pt-Co units)

Turbidity (NTU)

ANALYSIS OF SELECTED TRACE ELEMENTS

(Total concentrations in micrograms per liter, except as indicated)

$\begin{array}{lrlc}\text { Arsenic (As) } & 1 & \text { Manganese (Mn) } & 10 \\ \text { Barium (Ba) } & -- & \text { Mercury (Hg) } & <.5 \\ \text { Cadmium (Cd) } & 0 & \text { Selenium (Se) } & 0 \\ \text { Chromium (Cr) } & <10 & \text { Silver (Ag) } & 0 \\ \text { Copper (Cu) } & 3 & \text { Strontium, dissolved (Sr) } & 230 \\ \text { Iron (Fe) } & 20 & \text { Zinc ( } \mathrm{Cn}) & 10 \\ \text { Lead (Pb) } & 9 & & \end{array}$

ANALYSIS OF SELECTED PESTICIDE AND INDUSTRIAL COMPOUNDS

(Total concentrations in micrograms per liter)

$\begin{array}{llll}\text { Aldrin } & .00 & \text { Lindane } & .00 \\ \text { Chlordane } & .0 & \text { Methoxychlor } & .00 \\ \text { DDD } & .00 & \text { Mirex } & .00 \\ \text { DDE } & .00 & \text { PCB } & .0 \\ \text { DDT } & .00 & \text { PCN } & .00 \\ \text { Dieldrin } & .00 & \text { Silvex } & .00 \\ \text { Endrin } & .00 & \text { Toxaphene } & 0 \\ \text { Heptachlor } & .00 & 2,4-D & .00 \\ \text { Heptachlor epoxide } & .00 & 2,4,5-T & .00\end{array}$

ANALYSIS OF SELECTED GROSS RADIOACTIVITY

(Total activity concentrations in picocuries per liter)

Gross Alpha, as

uranium natural --

Gross Beta, as

cesium-137
Gross Beta, as

strontium -90 
PUBLIC WATER SUPPLY: Port St. Joe

COUNTY: Gulf

COLLECTION DATE: 12-23-77

SAMPLING POINT (1) Treated water-294911085180490, tap northwest corner of

City Hall.

(2) Raw water-294911085180400, intake canal from Chipola River, straining barrier bridge, mid point.

ANALYSIS OF MAJOR CHEMICAL CONSTITUENTS AND PHYSICAL PROPERTIES

(Dissolved concentrations in milligrams per liter, except as indicated)

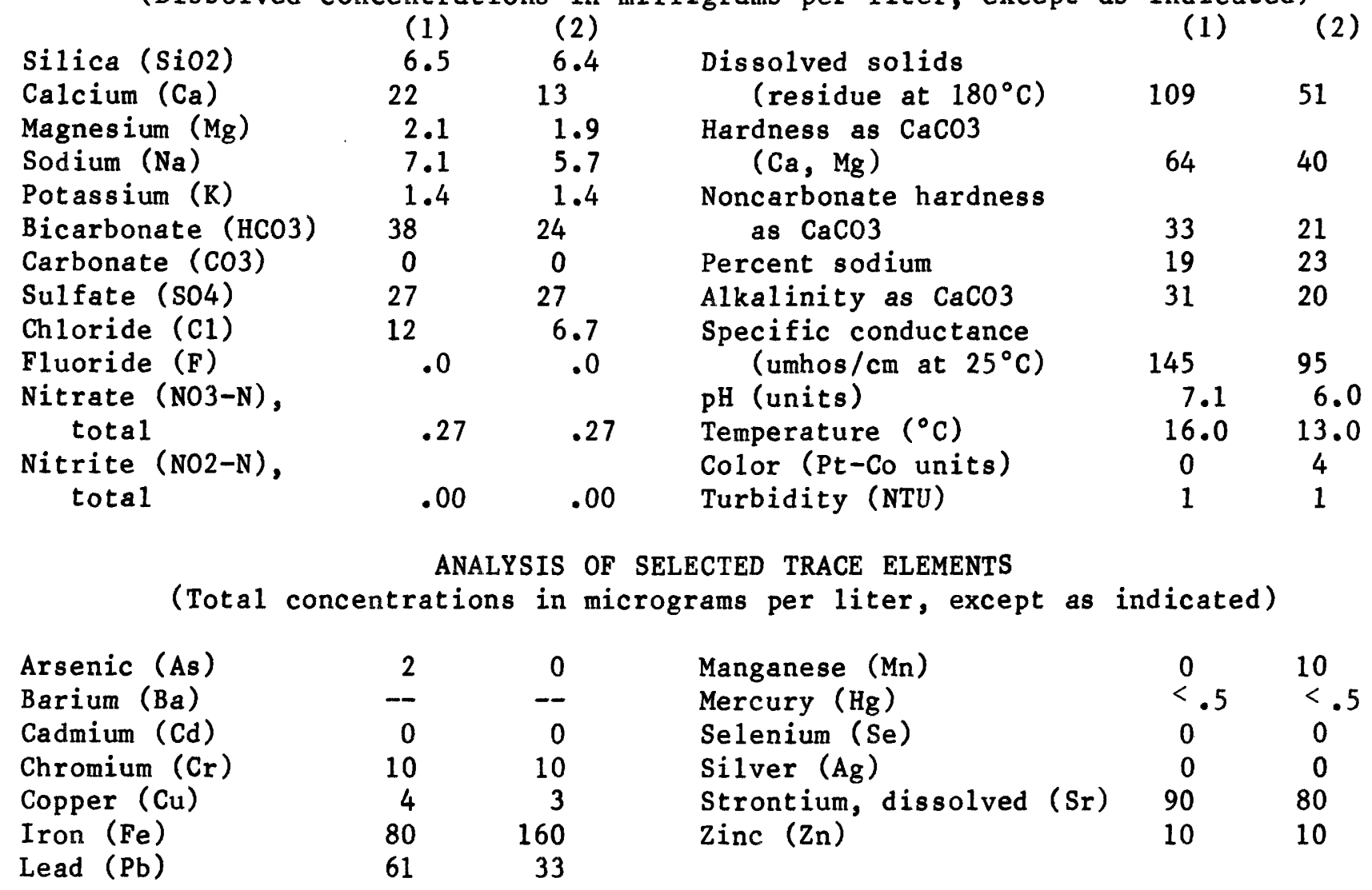

ANALYSIS OF SELECTED PESTICIDE AND INDUSTRIAL COMPOUNDS (Total concentrations in micrograms per liter)

$\begin{array}{llllll}\text { Aldrin } & .00 & .00 & \text { Lindane } & .00 & .00 \\ \text { Chlordane } & .0 & .0 & \text { Methoxychlor } & .00 & .00 \\ \text { DDD } & .00 & .00 & \text { Mirex } & .00 & .00 \\ \text { DDE } & .00 & .00 & \text { PCB } & .0 & .0 \\ \text { DDT } & .00 & .00 & \text { PCN } & .00 & .00 \\ \text { Dieldrin } & .00 & .00 & \text { Silvex } & .00 & .00 \\ \text { Endrin } & .00 & .00 & \text { Toxaphene } & 0 & 0 \\ \text { Heptachlor } & .00 & .00 & 2,4-D & .00 & .00 \\ \text { Heptachlor epoxide } & .00 & .00 & 2,4,5-T & .00 & .00\end{array}$

ANALYSIS OF SELECTED GROSS RADIOACTIVITY

(Total activity concentrations in picocuries per liter)

Gross Alpha, as

uranium natural

Gross Beta, as

cesium-137
$<1.2<1.1$

$2.0 \quad 1.7$
Gross Beta, as

strontium -90
$1.7 \quad 1.5$ 
PUBLIC WATER SUPPLY: Punta Gorda COUNTY: Charlotte

COLLECTION DATE: $12-22-77$

SAMPLING POINT (1) Treated water-265832081580709, tap inside water treatment plant.

(2) Raw water-265904081560901, Shel1 Creek near Punta Gorda.

ANALYSIS OF MAJOR CHEMICAL CONSTITUENTS AND PHYSICAL PROPERTIES

(Dissolved concentrations in milligrams per liter, except as indicated)
(1)
(2)
(1)

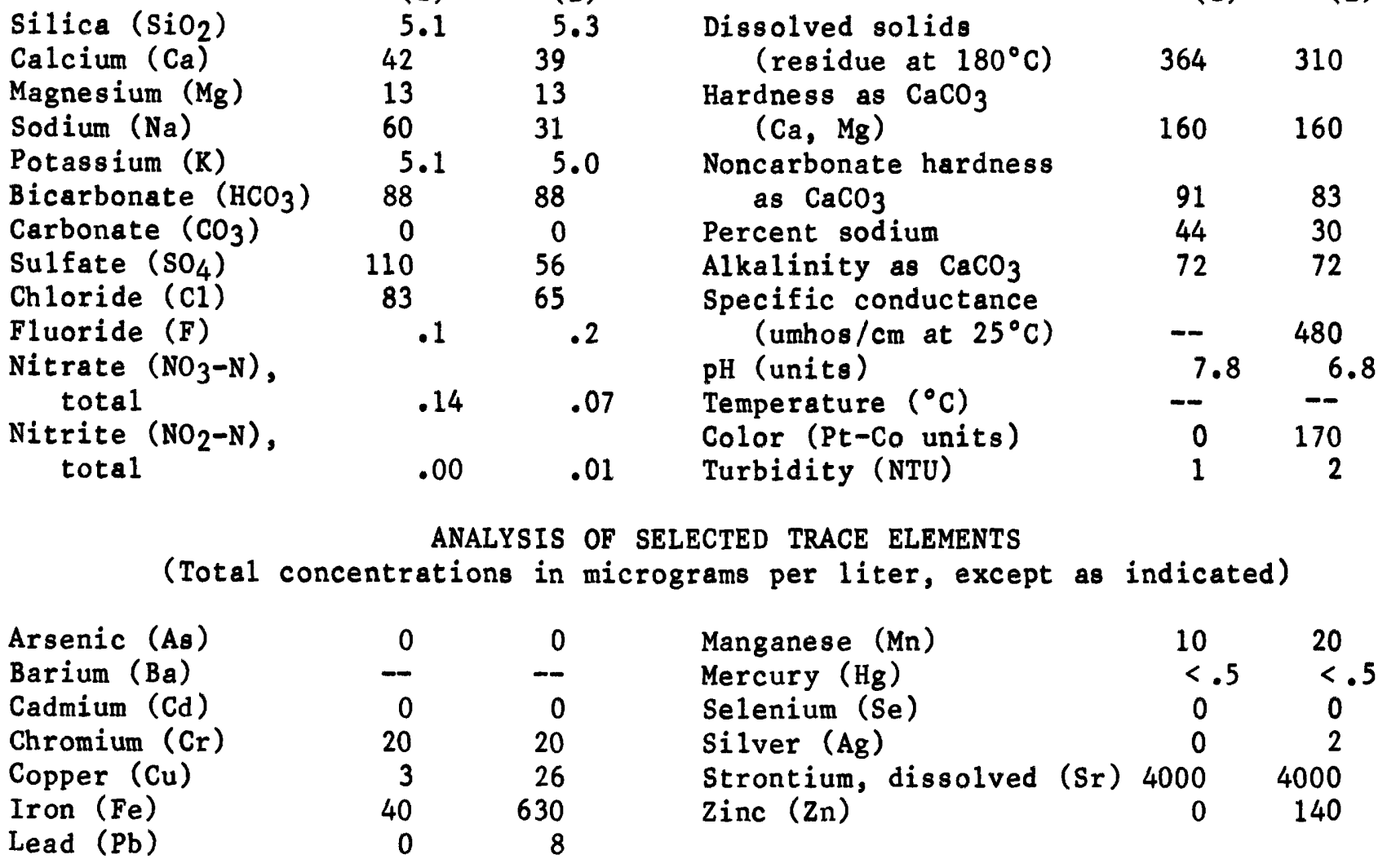

ANALYSIS OF SELECTED PESTICIDE AND INDUSTRIAL COMPOUNDS

(Total concentrations in micrograms per 1 iter)

$\begin{array}{llllll}\text { Aldrin } & .00 & .00 & \text { Lindane } & .00 & .00 \\ \text { Chlordane } & .0 & .0 & \text { Methoxych1or } & .00 & .00 \\ \text { DDD } & .00 & .00 & \text { Mirex } & .00 & .00 \\ \text { DDE } & .00 & .00 & \text { PCB } & .0 & .0 \\ \text { DDT } & .00 & .00 & \text { PCN } & .00 & .00 \\ \text { Dieldrin } & .00 & .00 & \text { Silvex } & .00 & .00 \\ \text { Endrin } & .00 & .00 & \text { Toxaphene } & 0 & 0 \\ \text { Heptachlor } & .00 & .00 & 2,4-D & .00 & .00 \\ \text { Heptachlor epoxide } & .00 & .00 & 2,4,5-T & .00 & .00\end{array}$

ANALYSIS OF SELECTED GROSS RADIOACTIVITY

(Total activity concentrations in picocuries per 1iter)

Grose Alpha, as uranium natura Gross Beta, as cesium-137
$<3.3 \quad 4.7$

10
Gross Beta, as

strontium-90

9.510 
PUBLIC WATER SUPPLY: Quincy

COUNTY: Gadsden

COLLECTION DATE: 11-22-77

SAMPLING POINT (1) Treated water-303554084344890, tap from water treatment plant settling tank.

(2) Raw water-02329534, Quincy Creek at State Road 267.

ANALYSIS OF MAJOR CHEMICAL CONSTITUENTS AND PHYSICAL PROPERTIES

(Dissolved concentrations in milligrams per liter, except as indicated)

(1) (2)

$5.8 \quad 6.0 \quad$ Dissolved solids

Silica (SiO2)

11

Calcium ( $\mathrm{Ca}$ )

Magnesium $(\mathrm{Mg})$

Sodium ( $\mathrm{Na}$ )

$1.4 \quad 1.4$

$3.4 \quad 4.7$

Potassium (K)

- 7

Bicarbonate ( $\mathrm{HCO} 3$ )

30

.7

Carbonate ( $\mathrm{CO} 3$ )

Sulfate ( 504 )

Chloride (Cl)

Fluoride (F)

0

19

$6.4 \quad .6$

$10 \quad 5.1$

Nitrate (NO3-N),

.1

5.1

total

Nitrite (NO2-N), total

$\begin{array}{ll}.38 & .38 \\ .00 & .00\end{array}$

(residue at $180^{\circ} \mathrm{C}$ )

(1)

Hardness as $\mathrm{CaCO} 3$

( $\mathrm{Ca}, \mathrm{Mg}$ )

Noncarbonate hardness as $\mathrm{CaCO} 3$

Percent sodium

\section{9}

18

0

Alkalinity as $\mathrm{CaCO} 3$

25

38

Specific conductance

(umhos $/ \mathrm{cm}$ at $25^{\circ} \mathrm{C}$ )

pH (units)

92

Temperature $\left({ }^{\circ} \mathrm{C}\right)$

Color (Pt-Co units)

Turbidity (NTU)

ANALYSIS OF SELECTED TRACE ELEMENTS

(Total concentrations in micrograms per liter, except as indicated)

$\begin{array}{lrr}\text { Arsenic (As) } & 0 & 1 \\ \text { Barium (Ba) } & 0 & 0 \\ \text { Cadmium (Cd) } & 2 & 2 \\ \text { Chromium (Cr) } & 10 & <10 \\ \text { Copper (Cu) } & 69 & 2 \\ \text { Iron (Fe) } & 30 & 1000 \\ \text { Lead (Pb) } & 49 & 20\end{array}$

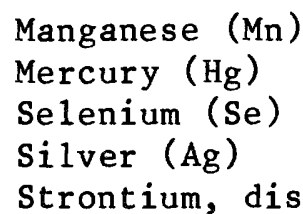

Strontium, dissolved (Sr)

Zinc $(\mathrm{Zn})$

$\begin{array}{cc}20 & 40 \\ <.5 & <.5 \\ 0 & 0 \\ 1 & 0 \\ 40 & 30 \\ 20 & 0\end{array}$

ANALYSIS OF SELECTED PESTICIDE AND INDUSTRIAL COMPOUNDS

(Total concentrations in micrograms per liter)

$\begin{array}{llll}\text { Aldrin } & .00 & .00 & \text { Lindane } \\ \text { Chlordane } & .0 & .0 & \text { Methoxych1or } \\ \text { DDD } & .00 & .00 & \text { Mirex } \\ \text { DDE } & .00 & .00 & \text { PCB } \\ \text { DDT } & .00 & .00 & \text { PCN } \\ \text { Dieldrin } & .00 & .00 & \text { Silvex } \\ \text { Endrin } & .00 & .00 & \text { Toxaphene } \\ \text { Heptachlor } & .00 & .00 & 2,4-D \\ \text { Heptachlor epoxide } & .00 & .00 & 2,4,5-T\end{array}$

$\begin{array}{ll}.00 & .00 \\ .00 & .00 \\ .00 & .00 \\ .0 & .0 \\ .00 & .00 \\ .00 & .00 \\ 0 & 0 \\ .00 & .00 \\ .00 & .00\end{array}$

ANALYSIS OF SELECTED GROSS RADIOACTIVITY

(Total activity concentrations in picocuries per liter)

Gross Alpha, as

uranium natural $<.5 \quad-$

Gross Beta, as

cesium-137
Gross Beta, as

strontium -90 
PUBLIC WATER SUPPLY: Rex Utilities (Florida City)

COUNTY: Dade COLLECTION DATE: 11-30-77

SAMPLING POINT (1) Treated water-252502080300401, tap outside administration building at Florida City Prison Camp.

(2) Raw water-

ANALYSIS OF MAJOR CHEMICAL CONSTITUENTS AND PHYSICAL PROPERTIES

(Dissolved concentrations in milligrams per liter, except as indicated)

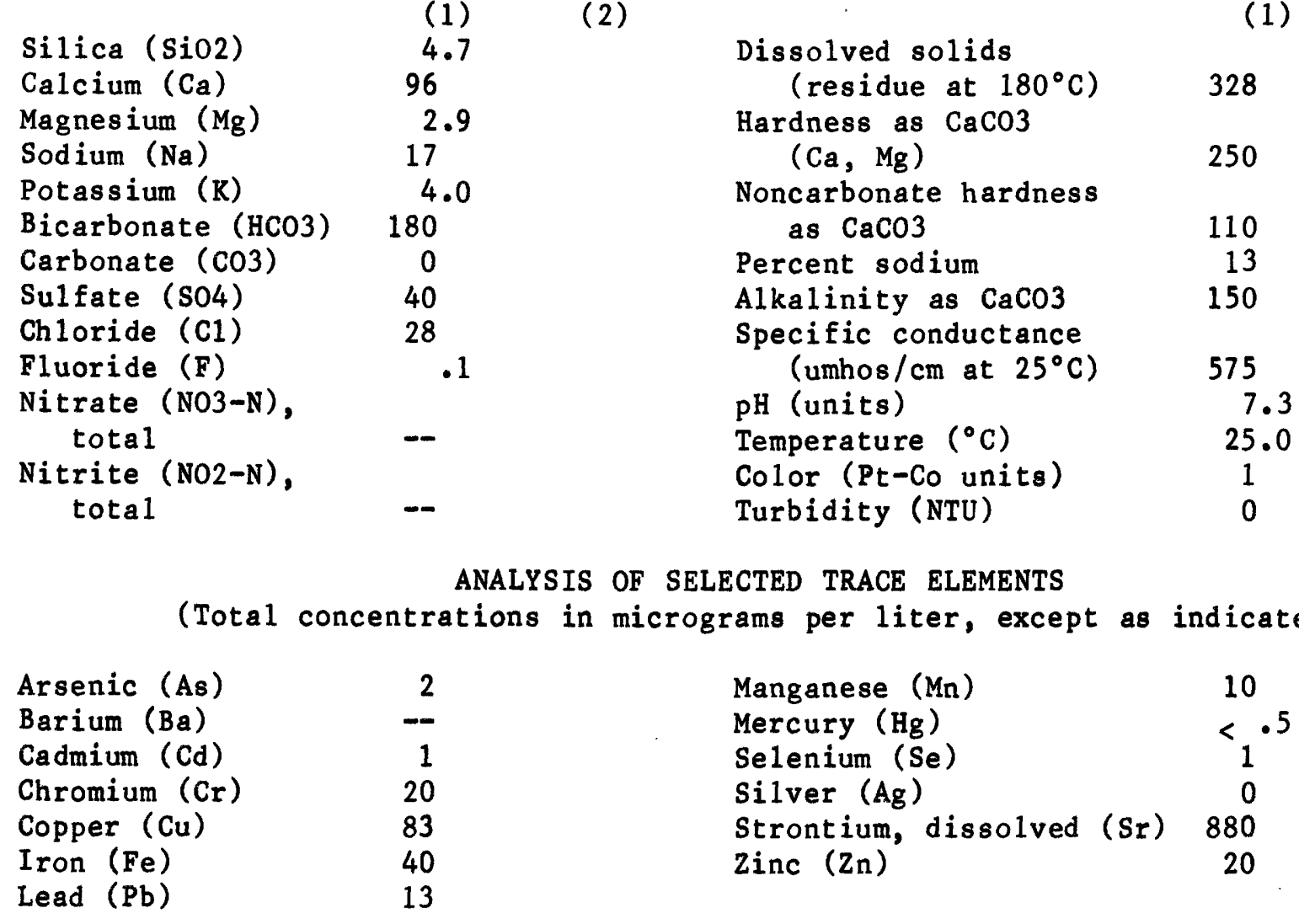

ANALYSIS OF SELECTED PESTICIDE AND INDUSTRIAL COMPOUNDS

(Total concentrations in micrograms per liter)

$\begin{array}{llll}\text { Aldrin } & .00 & \text { Lindane } & .00 \\ \text { Chlordane } & .0 & \text { Methoxychlor } & .00 \\ \text { DDD } & .00 & \text { Mirex } & .00 \\ \text { DDE } & .00 & \text { PCB } & .0 \\ \text { DDT } & .00 & \text { PCN } & .00 \\ \text { Dieldrin } & .00 & \text { Silvex } & .00 \\ \text { Endrin } & .00 & \text { Toxaphene } & 0 \\ \text { Heptachlor } & .00 & 2,4-D & .00 \\ \text { Heptachlor epoxide } & .00 & 2,4,5-T & .00\end{array}$

ANALYSIS OF SELECTED GROSS RADIOACTIVITY

(Total activity concentrations in picocuries per liter)

Gross Alpha, as uranium natural

Gross Beta, as

cesium-137
Gross Beta, as

strontium-90 
PUBLIC WA TER UPPLY: Ridge Manor

COUNTY: Hernando

COLLECTION DATE: 11-02-77

SAMPLING POINT (1) Treated water-283036082104809, tap on north side of pumphouse building.

(2) Raw water-

ANALYSIS OF MAJOR CHEMICAL CONSTITUENTS AND PHYSICAL PROPERTIES

(Dissolved concentrations in milligrams per liter, except as indicated)

(1) (2)

$\begin{array}{lc}\text { Silica (SiO2) } & 10 \\ \text { Calcium (Ca) } & 54 \\ \text { Magnesium (Mg) } & 1.0 \\ \text { Sodium (Na) } & 2.8 \\ \text { Potassium (K) } & .2 \\ \text { Bicarbonate (HCO3) } & 160 \\ \text { Carbonate (CO3) } & 0 \\ \text { Sulfate (SO4) } & .3 \\ \text { Chloride (C1) } & 5.4 \\ \text { Fluoride (F) } & .1 \\ \text { Nitrate (NO3-N), } & .28 \\ \quad \text { total (NO2-N), } \\ \text { Nitrite (Notal }\end{array}$

Dissolved solids (residue at $180^{\circ} \mathrm{C}$ ) $\quad 157$

Hardness as $\mathrm{CaCO} 3$ ( $\mathrm{Ca}, \mathrm{Mg}$ ) 140

Noncarbonate hardness as $\mathrm{CaCO} 3$

Percent sodium

Alkalinity as $\mathrm{CaCO} 3 \quad 130$

Specific conductance

(1) (umhos $/ \mathrm{cm}$ at $25^{\circ} \mathrm{C}$ )

$\mathrm{pH}$ (units)

$-\overline{7.5}$

Temperature $\left({ }^{\circ} \mathrm{C}\right)$

Color (Pt-Co units)

Turbidity (NTU)

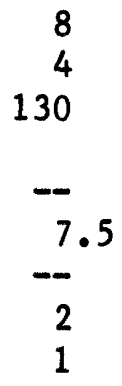

ANALYSIS OF SELECTED TRACE ELEMENTS

(Total concentrations in micrograms per liter, except as indicated)

$\begin{array}{lr}\text { Arsenic (As) } & 1 \\ \text { Barium ( } \mathrm{Ba} \text { ) } & - \\ \text { Cadmium ( } \mathrm{Cd}) & 0 \\ \text { Chromium (Cr) } & 20 \\ \text { Copper (Cu) } & 14 \\ \text { Iron (Fe) } & 160 \\ \text { Lead ( } \mathrm{Pb}) & 13\end{array}$

$\begin{array}{lc}\text { Manganese (Mn) } & 0 \\ \text { Mercury ( } \mathrm{Hg}) & <.5 \\ \text { Selenium ( } \mathrm{Se}) & 2 \\ \text { Silver ( } \mathrm{Ag}) & 0 \\ \text { Strontium, dissolved ( } \mathrm{Sr}) & 130 \\ \text { Zinc ( } \mathrm{Zn}) & 10\end{array}$

ANALYSIS OF SELECTED PESTICIDE AND INDUSTRIAL COMPOUNDS

( Total concentrations in micrograms per 1iter)

$\begin{array}{llll}\text { Aldrin } & .00 & \text { Lindane } & .00 \\ \text { Chlordane } & .0 & \text { Methoxych1or } & .00 \\ \text { DDD } & .00 & \text { Mirex } & .00 \\ \text { DDE } & .00 & \text { PCB } & .0 \\ \text { DDT } & .00 & \text { PCN } & .00 \\ \text { Dieldrin } & .00 & \text { Silvex } & .00 \\ \text { Endrin } & .00 & \text { Toxaphene } & 0 \\ \text { Heptachlor } & .00 & 2,4-D & .00 \\ \text { Heptachlor epoxide } & .00 & 2,4,5-T & .00\end{array}$

ANALYSIS OF SELECTED GROSS RADIOACTIVITY

(Total activity concentrations in picocuries per liter)

Gross Alpha, as

uranium natural --

Gross Beta, as

cesium-137
Gross Beta, as

strontium-90 
PUBLIC WATER SUPPLY: Ridge Utilities

COUNTY: Polk

COLLECTION DATE: 12-14-77

SAMPLING POINT (1) Treated water-281202081380809, tap outside pumphouse on east side (near Haines City).

(2) Raw water-

ANALYSIS OF MAJOR CHEMICAL CONSTITUENTS AND PHYSICAL PROPERTIES

(Dissolved concentrations in milligrams per liter, except as indicated)

$\begin{array}{lc}\text { Silica (SiO2) } & (1) \\ \text { Calcium ( } \mathrm{Ca}) & 38 \\ \text { Magnesium (Mg) } & 6.2 \\ \text { Sodium (Na) } & 6.7 \\ \text { Potassium (K) } & 1.1 \\ \text { Bicarbonate (HCO3) } & 140 \\ \text { Carbonate (CO3) } & 0 \\ \text { Sulfate (SO4) } & 4.9 \\ \text { Chloride (C1) } & 12 \\ \text { Fluoride (F) } & .1 \\ \text { Nitrate (NO3-N), } & .69 \\ \quad \text { tota1 Nitrite (NO2-N), } \\ \quad \text { total }\end{array}$

(2)

(1)

Dissolved solids

(residue at $180^{\circ} \mathrm{C}$ ) $\quad 159$

Hardness as $\mathrm{CaCO} 3$

$(\mathrm{Ca}, \mathrm{Mg})$

Noncarbonate hardness as $\mathrm{CaCO} 3$

Percent sodium

Alkalinity as $\mathrm{CaCO} 3$

Specific conductance

(umhos $/ \mathrm{cm}$ at $25^{\circ} \mathrm{C}$ )

$\mathrm{pH}$ (units)

Temperature $\left({ }^{\circ} \mathrm{C}\right)$

Color (Pt-Co units)

Turbidity (NTU)

\section{6}

11

110

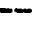

7.2

0

0

ANALYSIS OF SELECTED TRACE ELEMENTS

(Total concentrations in micrograms per liter, except as indicated)

$\begin{array}{lr}\text { Arsenic (As) } & 0 \\ \text { Barium (Ba) } & - \\ \text { Cadmium (Cd) } & 0 \\ \text { Chromium (Cr) } & 10 \\ \text { Copper (Cu) } & 8 \\ \text { Iron (Fe) } & 90 \\ \text { Lead (Pb) } & 7\end{array}$

Manganese (Mn)

0

Mercury ( $\mathrm{Hg}$ )

Selenium ( $\mathrm{Se}$ )

$<.5$

Silver (Ag)

Strontium, dissolved ( $\mathrm{Sr}$ ) 60

8

Zinc $(\mathrm{Zn})$

ANALYSIS OF SELECTED PESTICIDE AND INDUSTRIAL COMPOUNDS

( $T o t a l$ concentrations in micrograms per liter)

$\begin{array}{llll}\text { Aldrin } & .00 & \text { Lindane } & .00 \\ \text { Chlordane } & .0 & \text { Methoxych1or } & .00 \\ \text { DDD } & .00 & \text { Mirex } & .00 \\ \text { DDE } & .00 & \text { PCB } & .0 \\ \text { DDT } & .00 & \text { PCN } & .00 \\ \text { Dieldrin } & .00 & \text { Silvex } & .00 \\ \text { Endrin } & .00 & \text { Toxaphene } & 0 \\ \text { Heptachlor } & .00 & 2,4-D & .00 \\ \text { Heptachlor epoxide } & .00 & 2,4,5-\mathrm{T} & .00\end{array}$

ANALYSIS OF SELECTED GROSS RADIOACTIVITY

(Total activity concentrations in picocuries per liter)

Gross Alpha, as uranium natural

Gross Beta, as

cesium-137
Gross Beta, as

strontium-90

2.8 
PUBLIC WATER SUPPLY: Rotonda West COUNTY: Charlotte COLLECTION DATE: 12-22-77

SAMPLING POINT (1) Treated water -265105082164809 , tap at water treatment p1ant.

(2) Raw water-

ANALYSIS OF MAJOR CHEMICAL CONSTITUENTS AND PHYSICAL PROPERTIES (Dissolved concentrations in milligrams per liter, except as indicated)

$\begin{array}{lc}\text { Silica (SiO2) } & (1) \\ \text { Calcium (Ca) } & .9 \\ \text { Magnesium (Mg) } & 6.2 \\ \text { Sodium (Na) } & 5.3 \\ \text { Potassium (K) } & 140 \\ \text { Bicarbonate (HCO3) } & 3.1 \\ \text { Carbonate (CO3) } & 36 \\ \text { Sulfate (SO4) } & 0 \\ \text { Chloride (C1) } & 13 \\ \text { Fluoride (F) } & 210 \\ \text { Nitrate (NO3-N), } & .0 \\ \quad \text { total (NO2-N), } & .02 \\ \text { Nitrite (NO2-N) } & \\ \quad \text { total } & \end{array}$

(2)

(1)

Dissolved solids (residue at $180^{\circ} \mathrm{C}$ ) 378

Hardness as $\mathrm{CaCO} 3$ $(\mathrm{Ca}, \mathrm{Mg}) \quad 38$

Noncarbonate hardness as $\mathrm{CaCO} 3$

Percent sodium 88

Alkalinity as $\mathrm{CaCO} 3 \quad 30$

.0

Specific conductance (umhos $/ \mathrm{cm}$ at $25^{\circ} \mathrm{C}$ )

pH (units)

Temperature $\left({ }^{\circ} \mathrm{C}\right)$

Color (Pt-Co units)

Turbidity (NTU)

ANALYSIS OF SELECTED TRACE ELEMENTS

(Total concentrations in micrograms per liter, except as indicated)

$\begin{array}{lrlc}\text { Arsenic (As) } & 4 & \text { Manganese (Mn) } & 0 \\ \text { Barium (Ba) } & - & \text { Mercury (Hg) } & <.5 \\ \text { Cadmium (Cd) } & 0 & \text { Selenium ( } \mathrm{Se}) & 0 \\ \text { Chromium (Cr) } & <10 & \text { Silver (Ag) } & 0 \\ \text { Copper (Cu) } & 6 & \text { Strontium, dissolved ( } \mathrm{Sr} \text { ) } & 720 \\ \text { Iron (Fe) } & 50 & \text { Zinc ( } \mathrm{n} \text { ) } & 20 \\ \text { Lead (Pb) } & 0 & & \end{array}$

ANALYSIS OF SELECTED PESTICIDE AND INDUSTRIAL COMPOUNDS

(Total concentrations in micrograms per liter)

$\begin{array}{llll}\text { Aldrin } & .00 & \text { Lindane } & .00 \\ \text { Chlordane } & .0 & \text { Methoxychlor } & .00 \\ \text { DDD } & .00 & \text { Mirex } & .00 \\ \text { DDE } & .00 & \text { PCB } & .0 \\ \text { DDT } & .00 & \text { PCN } & .00 \\ \text { Dieldrin } & .00 & \text { Silvex } & .00 \\ \text { Endrin } & .00 & \text { Toxaphene } & 0 \\ \text { Heptachlor } & .00 & 2,4-D & .00 \\ \text { Heptachlor epoxide } & .00 & 2,4,5-T & .00\end{array}$

ANALYSIS OF SELECTED GROSS RADIOACTIVITY

(Total activity concentrations in picocuries per liter)

Gross Alpha, as uranium natural --

Gross Beta, as

cesium-137
Gross Beta, as

strontium -90 
PUBLIC WATER SUPPLY: Ruskin

COUNTY: Hillsborough

COLLECTION DATE: 12-20-77

SAMPLING POINT (1) Treated water-274239082260009, tap outside water treatment plant on east side of building.

(2) Raw water-

ANALYSIS OF MAJOR CHEMICAL CONSTITUENTS AND PHYSICAL PROPERTIES

(Dissolved concentrations in milligrams per liter, except as indicated)

(1)

Silica (SiO2)

Calcium ( $\mathrm{Ca})$

Magnesium ( $\mathrm{Mg}$ )

Sodium ( $\mathrm{Na}$ )

Potassium ( $K$ )

Bicarbonate ( $\mathrm{HCO} 3$ )

Carbonate ( $\mathrm{CO} 3)$

Sulfate (s04)

Chloride (C1)

Fluoride (F)

Nitrate (NO3-N), total

Nitrite (NO2-N),

total
23

110

45

12

1.8

180

0

300

24

.5

.03

.00
(2)

Dissolved solids

(residue at $180^{\circ} \mathrm{C}$ )

Hardness as $\mathrm{CaCO} 3$

( $\mathrm{Ca}, \mathrm{Mg}$ )

629

470

Noncarbonate hardness as $\mathrm{CaCO} 3$

320

Percent sodium

Alkalinity as $\mathrm{CaCO} 3$

Specific conductance

(umhos $/ \mathrm{cm}$ at $25^{\circ} \mathrm{C}$ )

$\mathrm{pH}$ (units)

Temperature $\left({ }^{\circ} \mathrm{C}\right)$

Color (Pt-Co units)

Turbidity (NTU)
(1)

ANALYSIS OF SELECTED TRACE ELEMENTS

(Total concentrations in micrograms per liter, except as indicated)

$\begin{array}{lrlr}\text { Arsenic (As) } & 0 & \text { Manganese (Mn) } & 10 \\ \text { Barium (Ba) } & -- & \text { Mercury (Hg) } & <.5 \\ \text { Cadmium (Cd) } & 0 & \text { Selenium ( Se) } & 0 \\ \text { Chromium (Cr) } & 10 & \text { Silver (Ag) } & 2 \\ \text { Copper (Cu) } & 6 & \text { Strontium, dissolved ( } \mathrm{Sr}) & 6300 \\ \text { Iron (Fe) } & 130 & \text { Zinc ( } \mathrm{Cn}) & 230 \\ \text { Lead (Pb) } & 14 & & \end{array}$

ANALYSIS OF SELECTED PESTICIDE AND INDUSTRIAL COMPOUNDS

(Total concentrations in micrograms per liter)

$\begin{array}{llll}\text { Aldrin } & .00 & \text { Lindane } & .00 \\ \text { Chlordane } & .0 & \text { Methoxychlor } & .00 \\ \text { DDD } & .00 & \text { Mirex } & .00 \\ \text { DDE } & .00 & \text { PCB } & .0 \\ \text { DDT } & .00 & \text { PCN } & .00 \\ \text { Dieldrin } & .00 & \text { Silvex } & .00 \\ \text { Endrin } & .00 & \text { Toxaphene } & 0 \\ \text { Heptachlor } & .00 & 2,4-D & .00 \\ \text { Heptachlor epoxide } & .00 & 2,4,5-T & .00\end{array}$

ANALYSIS OF SELECTED GROSS RADIOACTIVITY

(Total activity concentrations in picocuries per liter)

Gross Alpha, as uranium natural $\quad 6.8$

Gross Beta, as

cesium-137
Gross Beta, as

strontium-90

4.3 
PUBLIC WATER SUPPLY: St. Augustine COUNTY: St. Johns

COLLECTION DATE: $12-12-77$

SAMPLING POINT (1) Treated water-295326081194902, tap in front of water

treatment plant office, about 10 feet from raw sampling point.

(2) Raw water-295326081194901, tap on transmission line before mixing basin at water treatment plant.

ANALYSIS OF MAJOR CHEMICAL CONSTITUENTS AND PHYSICAL PROPERTIES

(Dissolved concentrations in milligrams per liter, except as indicated)

(1)

Silica ( $\mathrm{SiO2})$
Calcium ( $\mathrm{Ca})$
Magnesium ( $\mathrm{Mg}$ )
Sodium ( $\mathrm{Na})$
Potassium (K)
Bicarbonate (HCO3)
Carbonate (CO3)
Sulfate ( $\mathrm{SO} 4)$
Chloride (C1)
Fluoride (F)
Nitrate (NO3-N)
total
Nitrite (NO2-N)
total

18

(2)

$60 \quad 170$

$23 \quad 3.9$

100

$3.7 \quad 1.4$

24

0

160

200

$\begin{array}{ll}.4 & .1 \\ .10 & .19 \\ .02 & .00\end{array}$

Dissolved solids

(residue at $180^{\circ} \mathrm{C}$ )

Hardness as $\mathrm{CaCO} 3$

$(\mathrm{Ca}, \mathrm{Mg})$

Noncarbonate hardness as $\mathrm{CaCO} 3$

Percent sodium

Alkalinity as $\mathrm{CaCO} 3$

Specific conductance (umhos $/ \mathrm{cm}$ at $25^{\circ} \mathrm{C}$ )

$\mathrm{pH}$ (units)

Temperature $\left({ }^{\circ} \mathrm{C}\right)$

Color (Pt-Co units)

Turbidity (NTU)
(1)

(2)

ANALYSIS OF SELECTED TRACE ELEMENTS

(Total concentrations in micrograms per liter, except as indicated)

\begin{tabular}{lrr} 
Arsenic (As) & \multicolumn{1}{c}{} & 2 \\
Barium (Ba) & -- & -- \\
Cadmium (Cd) & 0 & 0 \\
Chromium (Cr) & 10 & 10 \\
Copper (Cu) & 11 & 23 \\
Iron (Fe) & 20 & 2700 \\
Lead (Pb) & 4 & 62
\end{tabular}

Manganese (Mn)

Mercury ( $\mathrm{Hg}$ )

Selenium ( $\mathrm{Se}$ )

Silver $(\mathrm{Ag})$

Strontium, dissolved (Sr) 1700

Zinc $(\mathrm{Zn})$
564

517

250

440

230

47

20

120

9

320

$1020 \quad 860$

8.7

19.5

0

1
6.7

20.0

0

5

ANALYSIS OF SELECTED PESTICIDE AND INDUSTRIAL COMPOUNDS

(Total concentrations in micrograms per liter)

$\begin{array}{llllll}\text { Aldrin } & .00 & .00 & \text { Lindane } & .00 & .00 \\ \text { Chlordane } & .0 & .0 & \text { Methoxychlor } & .00 & .00 \\ \text { DDD } & .00 & .00 & \text { Mirex } & .00 & .00 \\ \text { DDE } & .00 & .00 & \text { PCB } & .0 & .0 \\ \text { DDT } & .00 & .00 & \text { PCN } & .00 & .00 \\ \text { Dieldrin } & .00 & .00 & \text { Silvex } & .00 & .00 \\ \text { Endrin } & .00 & .00 & \text { Toxaphene } & 0 & 0 \\ \text { Heptachlor } & .00 & .00 & 2,4-D & .00 & .00 \\ \text { Heptachlor epoxide } & .00 & .00 & 2,4,5-T & .00 & .00\end{array}$

ANALYSIS OF SELECTED GROSS RADIOACTIVITY

(Total activity concentrations in picocuries per liter)

Gross Alpha, as uranium natural Gross Beta, as

cesium-137
$8.2<4.3$

$3.4<2.2$
Gross Beta, as

strontium -90

$3.0<2.0$ 
PUBLIC WATER SUPPLY: St. Cloud

COUNTY: Osceola

SAMPLING POINT (1) Treated water-281456081171901, (composite wells 2 \& 3), tap inside pumphouse near elevated water tank.

(2) Raw water-

ANALYSIS OF MAJOR CHEMICAL CONSTITUENTS AND PHYSICAL PROPERTIES (Dissolved concentrations in milligrams per liter, except as indicated)

(1) (2)

$\begin{array}{lc}\text { Silica (SiO2) } & 14 \\ \text { Calcium (Ca) } & 55 \\ \text { Magnesium (Mg) } & 7.6 \\ \text { Sodium (Na) } & 11 \\ \text { Potassium (K) } & 1.4 \\ \text { Bicarbonate (HCO3) } & 160 \\ \text { Carbonate (CO3) } & 0 \\ \text { Sulfate (SO4) } & 41 \\ \text { Chloride (C1) } & 16 \\ \text { Fluoride (F) } & .7 \\ \text { Nitrate (NO3-N), } & \\ \quad \text { total (NO2-N), } & .03 \\ \text { Nitrite (NO2-Nal } & \\ \quad \text { total } & .00\end{array}$

(1)

Dissolved solids (residue at $180^{\circ} \mathrm{C}$ ) 240 Hardness as $\mathrm{CaCO} 3$ $(\mathrm{Ca}, \mathrm{Mg}) \quad 170$

Noncarbonate hardness as $\mathrm{CaCO} 3$ 39 Percent sodium 12 Alkalinity as $\mathrm{CaCO} 3 \quad 130$ Specific conductance (umhos $/ \mathrm{cm}$ at $25^{\circ} \mathrm{C}$ ) $\mathrm{pH}$ (units) Temperature $\left({ }^{\circ} \mathrm{C}\right)$ Color (Pt-Co units) Turbidity (NTU)

ANALYSIS OF SELECTED TRACE ELEMENTS

(Total concentrations in micrograms per liter, except as indicated)

$\begin{array}{lrlr}\text { Arsenic (As) } & 0 & \text { Manganese (Mn) } & 0 \\ \text { Barium (Ba) } & 100 & \text { Mercury (Hg) } & <.5 \\ \text { Cadmium (Cd) } & 0 & \text { Selenium (Se) } & 0 \\ \text { Chromium (Cr) } & <10 & \text { Silver (Ag) } & 0 \\ \text { Copper (Cu) } & 5 & \text { Strontium, dissolved (Sr) } & 1200 \\ \text { Iron (Fe) } & 70 & \text { Zinc ( } \mathrm{Cn}) & 20 \\ \text { Lead (Pb) } & 5 & & \end{array}$

ANALYSIS OF SELECTED PESTICIDE AND INDUSTRIAL COMPOUNDS

(Total concentrations in micrograms per liter)

$\begin{array}{llll}\text { Aldrin } & .00 & \text { Lindane } & .00 \\ \text { Chlordane } & .0 & \text { Methoxychlor } & .00 \\ \text { DDD } & .00 & \text { Mirex } & .00 \\ \text { DDE } & .00 & \text { PCB } & .0 \\ \text { DDT } & .00 & \text { PCN } & .00 \\ \text { Dieldrin } & .00 & \text { Silvex } & .00 \\ \text { Endrin } & .00 & \text { Toxaphene } & 0 \\ \text { Heptachlor } & .00 & 2,4-D & .00 \\ \text { Heptachlor epoxide } & .00 & 2,4,5-\mathrm{T} & .00\end{array}$

ANALYSIS OF SELECTED GROSS RADIOACTIVITY

(Total activity concentrations in picocuries per liter)

Gross Alpha, as

uranium natural $\quad 3.8$

Gross Beta, as

cesium-137
Gross Beta, as

strontium-90 
PUBLIC WATER SUPPLY: St. Marks

COUNTY: Waku1la

COLLECTION DATE: 11-23-77

SAMPLING POINT (1) Treated water-301148084130390, tap on front of City Hall (east side) near front door.

(2) Raw water-

ANALYSIS OF MAJOR CHEMICAL CONSTITUENTS AND PHYSICAL PROPERTIES (Dissolved concentrations in milligrams per liter, except as indicated)

$\begin{array}{lc}\text { Silica ( } & (1) \\ \text { Calcium ( } \mathrm{Ca}) & 6.8 \\ \text { Magnesium (Mg) } & 95 \\ \text { Sodium (Na) } & 4.8 \\ \text { Potassium (K) } & 3.3 \\ \text { Bicarbonate (HCO3) } & 310 \\ \text { Carbonate (CO3) } & 0 \\ \text { Sulfate ( SO4) } & 6.8 \\ \text { Chloride (C1) } & 6.8 \\ \text { Fluoride (F) } & -- \\ \text { Nitrate (NO3-N), } & \\ \quad \text { total (NO2-N), } \\ \text { Nitrite } \\ \quad \text { total }\end{array}$
(2)

(1)

Dissolved solids

(residue at $180^{\circ} \mathrm{C}$ ) 275

Hardness as $\mathrm{CaCO} 3$

$(\mathrm{Ca}, \mathrm{Mg})$

Noncarbonate hardness as $\mathrm{CaCO} 3$

Percent sodium

Alkalinity as $\mathrm{CaCO} 3$

Specific conductance

(umhos $/ \mathrm{cm}$ at $25^{\circ} \mathrm{C}$ )

$\mathrm{pH}$ (units)

7.5

Temperature $\left({ }^{\circ} \mathrm{C}\right)$

20.5

Color (Pt-Co units)

0

Turbidity (NTU)

2

ANALYSIS OF SELECTED TRACE ELEMENTS

(Total concentrations in micrograms per liter, except as indicated)

$\begin{array}{lrlr}\text { Arsenic (As) } & 1 & \text { Manganese (Mn) } & 20 \\ \text { Barium (Ba) } & 100 & \text { Mercury (Hg) } & .5 \\ \text { Cadmium (Cd) } & 1 & \text { Selenium (Se) } & 0 \\ \text { Chromium (Cr) } & 10 & \text { Silver (Ag) } & 0 \\ \text { Copper (Cu) } & 5 & \text { Strontium, dissolved (Sr) } 100 \\ \text { Iron (Fe) } & 30 & \text { Zinc (Zn) } & 70 \\ \text { Lead ( } \mathrm{Pb}) & 21 & & \end{array}$

ANALYSIS OF SELECTED PESTICIDE AND INDUSTRIAL COMPOUNDS

( Total concentrations in micrograms per liter)

$\begin{array}{llll}\text { Aldrin } & .00 & \text { Lindane } & .00 \\ \text { Chlordane } & .0 & \text { Methoxychlor } & .00 \\ \text { DDD } & .00 & \text { Mirex } & .00 \\ \text { DDE } & .00 & \text { PCB } & .0 \\ \text { DDT } & .00 & \text { PCN } & .00 \\ \text { Dieldrin } & .00 & \text { Silvex } & .00 \\ \text { Endrin } & .00 & \text { Toxaphene } & 0 \\ \text { Heptachlor } & .00 & 2,4-D & .00 \\ \text { Heptachlor epoxide } & .00 & 2,4,5-\mathrm{T} & .00\end{array}$

ANALYSIS OF SELECTED GROSS RADIOACTIVITY

(Total activity concentrations in picocuries per liter)

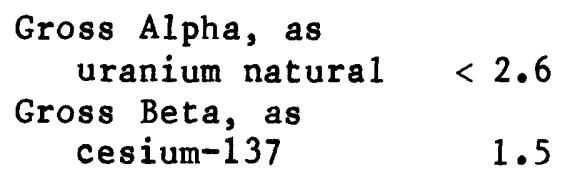


PUBLIC WATER SUPPLY: San intonio

COUNTY: Pasco

COLLECTION DATE: $12-20-77$

SAMPLING POINT (1) Treated watex-28201.1082162301, tap inside City Hal1.

(2) Raw watex

ANALYSIS OF MAJOR CHEMICAL CONSTITUENTS AND PHYSICAL PROPERTIES

(Dissolved concentrations in milligrams per liter, except as indicated)

$\begin{array}{lc}\text { Silica ( } & 9.82) \\ \text { Calcium (Ca) } & 50 \\ \text { Magnesium (Mg) } & 2.1 \\ \text { Sodium (Na) } & 14 \\ \text { Potassium (K) } & .5 \\ \text { Bicarbonate (HCO3) } & 150 \\ \text { Carbonate (CO3) } & 0 \\ \text { Sulfate (SO4) } & 4.2 \\ \text { Chloride (C1) } & 21 \\ \text { Fluoride (F) } & .0 \\ \text { Nitrate (NO3-N), } & \\ \quad \text { total (NO2-N), } & .72 \\ \text { Nitrite } \\ \quad \text { total }\end{array}$

(2)

(1)

Dissolved solids

(residue at $180^{\circ} \mathrm{C}$ ) $\quad 173$

Hardness as $\mathrm{CaCO} 3$

( $\mathrm{Ca}, \mathrm{Mg}$ )

130

Noncarbonate hardness

as $\mathrm{CaCO} 3$

11

Percent sodium 19

Alkalinity as $\mathrm{CaCO} 3 \quad 120$

Specific conductance

(umhos $/ \mathrm{cm}$ at $25^{\circ} \mathrm{C}$ )

$\mathrm{pH}$ (units)

Temperature $\left({ }^{\circ} \mathrm{C}\right)$

Color (Pt-Co units)

Turbidity (NTU)

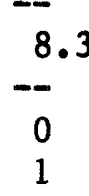

ANALYSIS OF SELECTED TRACE ELEMENTS

(Total concentrations in micrograms per liter, except as indicated)

$\begin{array}{lrlr}\text { Arsenic (As) } & 3 & \text { Manganese (Mn) } & 20 \\ \text { Barium (Ba) } & -- & \text { Mercury (Hg) } & <.5 \\ \text { Cadmium (Cd) } & 1 & \text { Selenium ( } \mathrm{Se}) & 0 \\ \text { Chromium (Cr) } & 30 & \text { Silver (Ag) } & 0 \\ \text { Copper (Cu) } & 13 & \text { Strontium, dissolved (Sr) } 100 \\ \text { Iron (Fe) } & 550 & \text { Zinc (Zn) } & 40 \\ \text { Lead ( } \mathrm{Pb}) & 15 & & \end{array}$

ANALYSIS OF SELECTED PESTICIDE AND INDUSTRIAL COMPOUNDS

(Total concentrations in micrograms per 1iter)

$\begin{array}{llll}\text { Aldrin } & .00 & \text { Lindane } & .00 \\ \text { Chlordane } & .0 & \text { Methoxych lor } & .00 \\ \text { DDD } & .00 & \text { Mirex } & .00 \\ \text { DDE } & .00 & \text { PCB } & .0 \\ \text { DDT } & .00 & \text { PCN } & .00 \\ \text { Dieldrin } & .00 & \text { Silvex } & .00 \\ \text { Endrin } & .00 & \text { Toxaphene } & 0 \\ \text { Heptachlor } & .00 & 2,4-D & .00 \\ \text { Heptachlor epoxide } & .00 & 2,4,5-T & .00\end{array}$

ANALYSIS OF SELECTED GROSS RADIOACTIVITY

(Total activity concentrations in picocuries per liter)

\footnotetext{
Gross Alpha, as

uranium natural

Gross Beta, as

cesium-137
}

Gross Beta, as

strontium -90 
PUBLIC WATER SUPPLY: Sarasota

COUNTY: Sarasota

COLLECTION DATE: 12-21-77

SAMPLING POINT (1) Treated water-272252082175409, tap outside water treatment plant.

(2) Raw water-

ANALYSIS OF MAJOR CHEMICAL CONSTITUENTS AND PHYSICAL PROPERTIES (Dissolved concentrations in milligrams per liter, except as indicated)

Silica (SiO2)

(1)

(2)

(1)

Calcium ( $\mathrm{Ca}$ )

Magnesium ( $\mathrm{Mg})$

Sodium ( $\mathrm{Na}$ )

Potassium (K)

Bicarbonate ( $\mathrm{HCO} 3$ )

Carbonate ( $\mathrm{CO} 3$ )

Sulfate ( 504 )

Chloride (C1)

Fluoride (F)

Nitrate $(\mathrm{NO3}-\mathrm{N})$,

total

Nitrite ( NO2-N),

total

Dissolved solids

(residue at $180^{\circ} \mathrm{C}$ )

Hardness as $\mathrm{CaCO} 3$

$(\mathrm{Ca}, \mathrm{Mg})$

Noncarbonate hardness

as $\mathrm{CaCO} 3$

Percent sodium

Alkalinity as $\mathrm{CaCO} 3$

Specific conductance

(umhos $/ \mathrm{cm}$ at $25^{\circ} \mathrm{C}$ )

$\mathrm{pH}$ (units)

Temperature $\left({ }^{\circ} \mathrm{C}\right)$

Color (Pt-Co units)

Turbidity (NTU)

ANALYSIS OF SELECTED TRACE ELEMENTS

(Total concentrations in micrograms per liter, except as indicated)

Arsenic (As)

Barium ( $\mathrm{Ba}$ )

Cadmium ( $C d$ )

Chromium ( $\mathrm{Cr}$ )

Copper $(\mathrm{Cu})$

Iron ( $\mathrm{Fe}$ )

Lead $(\mathrm{Pb})$
Manganese (Mn)

Mercury ( $\mathrm{Hg}$ )

Selenium ( $\mathrm{Se}$ )

Silver ( $\mathrm{Ag}$ )

Strontium, dissolved ( $S r)$

Zinc ( $\mathrm{Zn})$

ANALYSIS OF SELECTED PESTICIDE AND INDUSTRIAL COMPOUNDS

(Total concentrations in micrograms per 1iter)

Aldrin

Ch lordane

DDD

DDE

DDT

Dieldrin

Endrin

Heptach 1 or

Heptachlor epoxide
Lind ane

Methoxych 1 or

Mirex

PCB

PCN

Silvex

Toxaphene

2, 4-D

$2,4,5-T$

ANALYSIS OF SELECTED GROSS RADIOACTIVITY

(Total activity concentrations in picocuries per liter)

Gross Alpha, as

uranium natural $\quad 6.8$

Gross Beta, as

cesium-137
Gross Beta, as

strontium -90

7.4 
PUBLIC WATER SUPPLY: Sawgrass

COUNTY: St. Johns

COLLECTION DATE: 12-12-77

SAMPLING POINT (1) Treated water-301118081224401, tap on north side of holding tank about 30 feet from water treatment plant (building 10047) at Sawgrass development. (2) Raw water-

ANALYSIS OF MAJOR CHEMICAL CONSTITUENTS AND PHYSICAL PROPERTIES (Dissolved concentrations in milligrams per liter, except as indicated)

(1) (2)

$\begin{array}{lc}\text { Silica ( SiO2) } & 23 \\ \text { Calcium (Ca) } & 63 \\ \text { Magnesium (Mg) } & 34 \\ \text { Sodium (Na) } & 17 \\ \text { Potassium (K) } & 3.0 \\ \text { Bicarbonate (HCO3) } & 140 \\ \text { Carbonate (CO3) } & 0 \\ \text { Sulfate (SO4) } & 170 \\ \text { Chloride (C1) } & 24 \\ \text { F1uoride (F) } & .8 \\ \text { Nitrate (NO3-N), } & \\ \quad \text { total (NO2-N), } & .03 \\ \text { Nitrite } & \\ \quad \text { total }\end{array}$

Dissolved solids
$\quad$ (residue at $180^{\circ} \mathrm{C}$ )
Hardness as $\mathrm{CaCO} 3$
$\quad$ (Ca, Mg)
Noncarbonate hardnes
as CaCO3
Percent sodium
Alkalinity as CaCO3
Specific conductance
$\quad$ (umhos/cm at $25^{\circ} \mathrm{C}$ )
pH (units)
Temperature ( ${ }^{\circ} \mathrm{C}$ )
Color (Pt-Co units)
Turbidity (NTi)

(1)

ANALYSIS OF SELECTED TRACE ELEMENTS

(Total concentrations in micrograms per liter, except as indicated)

$\begin{array}{lrlr}\text { Arsenic (As) } & 2 & \text { Manganese (Mn) } & 10 \\ \text { Barium (Ba) } & -- & \text { Mercury (Hg) } & <.5 \\ \text { Cadmium (Cd) } & 0 & \text { Selenium (Se) } & 0 \\ \text { Chromium (Cr) } & 20 & \text { Silver (Ag) } & 1 \\ \text { Copper (Cu) } & 13 & \text { Strontium, dissolved (Sr) } 2100 \\ \text { Iron (Fe) } & 90 & \text { Zinc (Zn) } & 10 \\ \text { Lead ( } \mathrm{Pb}) & 6 & & \end{array}$

ANALYSIS OF SELECTED PESTICIDE AND INDUSTRIAL COMPOUNDS

(Total concentrations in micrograms per liter)

$\begin{array}{llll}\text { Aldrin } & .00 & \text { Lindane } & .00 \\ \text { Chlordane } & .0 & \text { Methoxychlor } & .00 \\ \text { DDD } & .00 & \text { Mirex } & .00 \\ \text { DDE } & .00 & \text { PCB } & .0 \\ \text { DDT } & .00 & \text { PCN } & .00 \\ \text { Dieldrin } & .00 & \text { Silvex } & .00 \\ \text { Endrin } & .00 & \text { Toxaphene } & 0 \\ \text { Heptachlor } & .00 & 2,4-D & .00 \\ \text { Heptachlor epoxide } & .00 & 2,4,5-T & .00\end{array}$

ANALYSIS OF SELECTED GROSS RADIOACTIVITY

(Total activity concentrations in picocuries per liter)

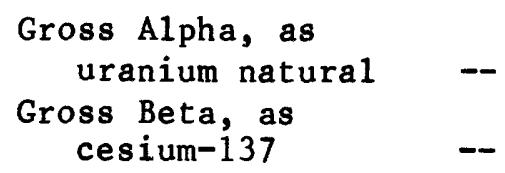

Gross Beta, as strontium -90 
PUBLIC WATER SUPPLY: Sneads

COUNTY: Jackson

COLLECTION DATE: $12-02-77$

SAMPLING POINT (1) Treated water-304315084555290, tap on west side of U.S. Post office building.

(2) Raw water-

ANALYSIS OF MAJOR CHEMICAL CONSTITUENTS AND PHYSICAL PROPERTIES (Dissolved concentrations in milligrams per liter, except as indicated)

(1) (2)

$\begin{array}{lc}\text { Silica (SiO2) } & 11 \\ \text { Calcium ( } \mathrm{Ca}) & 24 \\ \text { Magnesium (Mg) } & 12 \\ \text { Sodium (Na) } & 2.1 \\ \text { Potassium (K) } & .3 \\ \text { Bicarbonate (HCO3) } & 120 \\ \text { Carbonate (CO3) } & 0 \\ \text { Sulfate (SO4) } & 1.2 \\ \text { Chloride (C1) } & 3.7 \\ \text { Fluoride (F) } & .1 \\ \text { Nitrate (NO3-N), } & \\ \quad \text { total } & .82 \\ \text { Nitrite (NO2-N), } \\ \quad \text { total }\end{array}$

Dissolved solids (residue at $180^{\circ} \mathrm{C}$ )

(1)

115

Hardness as $\mathrm{CaCO} 3$

$(\mathrm{Ca}, \mathrm{Mg})$

Noncarbonate hardness

as $\mathrm{CaCO} 3$

Percent sodium

Alkalinity as $\mathrm{CaCO} 3$

Specific conductance

(umhos $/ \mathrm{cm}$ at $25^{\circ} \mathrm{C}$ )

$\mathrm{pH}$ (units)

Temperature $\left({ }^{\circ} \mathrm{C}\right)$

Color (Pt-Co units)

Turbidity (NTU)
110

11
4
98
180
7.8
20.0
1
0

ANALYSIS OF SELECTED TRACE ELEMENTS

(Total concentrations in micrograms per liter, except as indicated)

$\begin{array}{lr}\text { Arsenic (As) } & 0 \\ \text { Barium ( } \mathrm{Ba} \text { ) } & 0 \\ \text { Cadmium ( } \mathrm{Cd} \text { ) } & 0 \\ \text { Chromium (Cr) } & 10 \\ \text { Copper (Cu) } & 4 \\ \text { Iron (Fe) } & 30 \\ \text { Lead ( } \mathrm{Pb} \text { ) } & 9\end{array}$

$\begin{array}{lc}\text { Manganese (Mn) } & 0 \\ \text { Mercury (Hg) } & 1.2 \\ \text { Selenium ( Se) } & 0 \\ \text { Silver (Ag) } & 0 \\ \text { Strontium, dissolved ( } \mathrm{Sr}) & 60 \\ \text { Zinc (Zn) } & 20\end{array}$

ANALYSIS OF SELECTED PESTICIDE AND INDUSTRIAL COMPOUNDS ( Total concentrations in micrograms per liter)

$\begin{array}{llll}\text { Aldrin } & .00 & \text { Lindane } & .00 \\ \text { Chlordane } & .0 & \text { Methoxych1or } & .00 \\ \text { DDD } & .00 & \text { Mirex } & .00 \\ \text { DDE } & .00 & \text { PCB } & .0 \\ \text { DDT } & .00 & \text { PCN } & .00 \\ \text { Dieldrin } & .00 & \text { Silvex } & .00 \\ \text { Endrin } & .00 & \text { Toxaphene } & 0 \\ \text { Heptachlor } & .00 & 2,4-D & .00 \\ \text { Heptachlor epoxide } & .00 & 2,4,5-T & .00\end{array}$

ANALYSIS OF SELECTED GROSS RADIOACTIVITY

(Total activity concentrations in picocuries per liter)

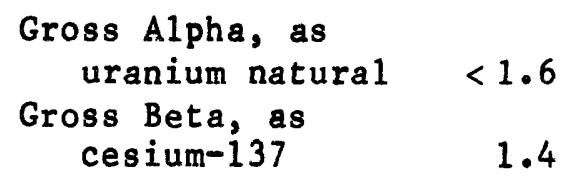


PUBLIC WATER SUPPLY: South Miami Heights

COLLECTION DATE: 12-15-77

SAMPLING POINT (1) Treated water-253427080193901, Tap at store, 20740 SW 84 th Avenue, Saga Bay.

(2) Raw water-

ANALYSIS OF MAJOR CHEMICAL CONSTITUENTS AND PHYSICAL PROPERTIES (Dissolved concentrations in milligrams per liter, except as indicated)

$\begin{array}{lc}\text { Silica (SiO2) } & 4.8 \\ \text { Calcium (Ca) } & 76 \\ \text { Magnesium (Mg) } & 2.0 \\ \text { Sodium (Na) } & 12 \\ \text { Potassium (K) } & 1.6 \\ \text { Bicarbonate (HCO3) } & 200 \\ \text { Carbonate (CO3) } & 0 \\ \text { Sulfate (SO4) } & 20 \\ \text { Chloride (C1) } & 22 \\ \text { Fluoride (F) } & .1 \\ \text { Nitrate (NO3-N), } & \\ \quad \text { total (N2-N), } & .44 \\ \text { Nitrite (NO2-N) } \\ \quad \text { total }\end{array}$

(2)

Dissolved solids

(residue at $180^{\circ} \mathrm{C}$ )

(1)

251

Hardness as $\mathrm{CaCO} 3$

( $\mathrm{Ca}, \mathrm{Mg}$ )

200

Noncarbonate hardness

as $\mathrm{CaCO} 3$

35

Percent sodium 12

Alkalinity as $\mathrm{CaCO} 3 \quad 160$

Specific conductance

(umhos $/ \mathrm{cm}$ at $25^{\circ} \mathrm{C}$ )

$\mathrm{pH}$ (units)

435

Temperature $\left({ }^{\circ} \mathrm{C}\right)$

Color (Pt-Co units)

Turbidity (NTU)

ANALYSIS OF SELECTED TRACE ELEMENTS

(Total concentrations in micrograms per liter, except as indicated)

$\begin{array}{lrlr}\text { Arsenic (As) } & 2 & \text { Manganese (Mn) } & 0 \\ \text { Barium (Ba) } & -- & \text { Mercury (Hg) } & <\cdot 5 \\ \text { Cadmium (Cd) } & 8 & \text { Selenium (Se) } & 0 \\ \text { Chromium (Cr) } & 10 & \text { Silver (Ag) } & 1 \\ \text { Copper (Cu) } & 110 & \text { Strontium, dissolved (Sr) } & 770 \\ \text { Iron (Fe) } & 90 & \text { Zinc ( } \mathrm{Cn}) & 10 \\ \text { Lead (Pb) } & 68 & & \end{array}$

ANALYSIS OF SELECTED PESTICIDE AND INDUSTRIAL COMPOUNDS (Total concentrations in micrograms per 1iter)

$\begin{array}{llll}\text { Aldrin } & .00 & \text { Lindane } & .00 \\ \text { Chlordane } & .0 & \text { Methoxychlor } & .00 \\ \text { DDD } & .00 & \text { Mirex } & .00 \\ \text { DDE } & .00 & \text { PCB } & .0 \\ \text { DDT } & .00 & \text { PCN } & .00 \\ \text { Die1drin } & .00 & \text { Silvex } & .00 \\ \text { Endrin } & .00 & \text { Toxaphene } & 0 \\ \text { Heptach1or } & .00 & 2,4-D & .00 \\ \text { Heptachlor epoxide } & .00 & 2,4,5-T & .00\end{array}$

ANALYSIS OF SELECTED GROSS RADIOACTIVITY

(Total activity concentrations in picocuries per liter)

Gross A1pha, as uranium natura1 --

Gross Beta, as

cesium-137
Gross Beta, as

strontium-90 
PUBLIC WATER SUPPLY: South Walton County Utilities

COUNTY: Walton

COLLECTION DATE: 01-05-78

SAMPLING POINT (1) Treated water-302241086223690, tap near left side of entrance to the Museum of the Sea and Indian.

(2) Raw water-

ANALYSIS OF MAJOR CHEMICAL CONSTITUENTS AND PHYSICAL PROPERTIES

(Dissolved concentrations in milligrams per liter, except as indicated)

(1) (2)

(1)

$\begin{array}{lc}\text { Silica (SiO2) } & 3.1 \\ \text { Calcium (Ca) } & 1.9 \\ \text { Magnesium (Mg) } & 2.1 \\ \text { Sodium (Na) } & 11 \\ \text { Potassium (K) } & .7 \\ \text { Bicarbonate (HCO3) } & 0 \\ \text { Carbonate (CO3) } & 0 \\ \text { Sulfate (SO4) } & 9.5 \\ \text { Chloride (C1) } & 18 \\ \text { Fluoride (F) } & .0 \\ \text { Nitrate (NO3-N), } & \\ \quad \text { total } & .00 \\ \text { Nitrite (NO2-N), } & \\ \quad \text { total }\end{array}$

Dissolved solids

(residue at $180^{\circ} \mathrm{C}$ ) $\quad 48$

Hardness as $\mathrm{CaCO} 3$

( $\mathrm{Ca}, \mathrm{Mg}$ )

13

Noncarbonate hardness as $\mathrm{CaCO} 3$

Percent sodium 63

Alkalinity as $\mathrm{CaCO} 3 \quad 0$

Specific conductance

(umhos $/ \mathrm{cm}$ at $25^{\circ} \mathrm{C}$ )

80

pH (units)

Temperature $\left({ }^{\circ} \mathrm{C}\right)$

Color (Pt-Co units)

Turbidity (NTU)

ANALYSIS OF SELECTED TRACE ELEMENTS

(Total concentrations in micrograms per liter, except as indicated)

$\begin{array}{lr}\text { Arsenic (As) } & 1 \\ \text { Barium (Ba) } & - \\ \text { Cadmium (Cd) } & 0 \\ \text { Chromium (Cr) } & 10 \\ \text { Copper (Cu) } & 8 \\ \text { Iron (Fe) } & 2400 \\ \text { Lead ( } \mathrm{Pb}) & 26\end{array}$

Manganese $(\mathrm{Mn})$

Mercury $(\mathrm{Hg})$

Selenium ( $\mathrm{Se}$ )

10

Silver ( $\mathrm{Ag}$ )

$<.5$

$\overline{0}$

Strontium, dissolved ( $\mathrm{Sr}$ ) 40

Zinc $(\mathrm{Zn})$

240

ANALYSIS OF SELECTED PESTICIDE AND INDUSTRIAL COMPOUNDS

(Total concentrations in micrograms per liter)

$\begin{array}{llll}\text { Aldrin } & .00 & \text { Lindane } & .00 \\ \text { Chlordane } & .0 & \text { Methoxychlor } & .00 \\ \text { DDD } & .00 & \text { Mirex } & .00 \\ \text { DDE } & .00 & \text { PCB } & .0 \\ \text { DDT } & .00 & \text { PCN } & .00 \\ \text { Dieldrin } & .00 & \text { Silvex } & .00 \\ \text { Endrin } & .00 & \text { Toxaphene } & 0 \\ \text { Heptachlor } & .00 & 2,4-D & .00 \\ \text { Heptachlor epoxide } & .00 & 2,4,5-T & .00\end{array}$

ANALYSIS OF SELECTED GROSS RADIOACTIVITY

( Total activity concentrations in picocuries per liter)

Gross Alpha, as

uranium natural

Gross Beta, as

cesium-137
Gross Beta, as

strontium-90 
PUBLIC WATER SUPPLY: Sunrise

COUNTY: Broward

COLLECTION DATE: $11-23-77$

SAMPLING POINT (1) Treated water-260948080180601, tap at house, 123rd Terrace and 29th Avenue.

(2) Raw water-

ANALYSIS OF MAJOR CHEMICAL CONSTITUENTS AND PHYSICAL PROPERTIES

(Dissolved concentrations in milligrams per liter, except as indicated)

$\begin{array}{lc} & (1) \\ \text { Silica (SiO2) } & 8.8 \\ \text { Calcium (Ca) } & 37 \\ \text { Magnesium (Mg) } & 1.9 \\ \text { Sodium (Na) } & 30 \\ \text { Potassium (K) } & 2.4 \\ \text { Bicarbonate (HCO3) } & 35 \\ \text { Carbonate (CO3) } & 0 \\ \text { Sulfate (SO4) } & 62 \\ \text { Chloride (C1) } & 55 \\ \text { Fluoride (F) } & .1 \\ \text { Nitrate (NO3-N), } & \\ \quad \text { tota1 (NO2-N), } \\ \text { Nitrite (Nota1 }\end{array}$

(2)

(1)

Dissolved solids

(residue at $180^{\circ} \mathrm{C}$ ) 210

Hardness as $\mathrm{CaCO} 3$

$(\mathrm{Ca}, \mathrm{Mg})$

Noncarbonate hardness as $\mathrm{CaCO}_{3} \quad 72$

Percent sodium 39

Alkalinity as $\mathrm{CaCO} 3 \quad 29$

Specific conductance

(umhos $/ \mathrm{cm}$ at $25^{\circ} \mathrm{C}$ ) $\quad 380$

pH (units)

Temperature $\left({ }^{\circ} \mathrm{C}\right)$

Color (Pt-Co units)

25.0

Turbidity (NTU)

4

1

ANALYSIS OF SELECTED TRACE ELEMENTS

(Total concentrations in micrograms per 1iter, except as indicated)

$\begin{array}{lr}\text { Arsenic ( } \mathrm{As} \text { ) } & 1 \\ \text { Barium (Ba) } & -- \\ \text { Cadmium ( } \mathrm{Cd}) & 1 \\ \text { Chromium (Cr) } & 20 \\ \text { Copper (Cu) } & 14 \\ \text { Iron (Fe) } & 50 \\ \text { Lead (Pb) } & 20\end{array}$

Manganese (Mn) 10

Mercury ( $\mathrm{Hg}$ ) $<.5$

Selenium ( $\mathrm{Se}$ ) 0

Silver ( $\mathrm{Ag})$

Strontium, dissolved ( $\mathrm{Sr}$ ) 550

Zinc $(\mathrm{Zn})$

ANALYSIS OF SELECTED PESTICIDE AND INDUSTRIAL COMPOUNDS

(Total concentrations in micrograms per 1iter)

$\begin{array}{llll}\text { Aldrin } & .00 & \text { Lindane } & .00 \\ \text { Chlordane } & .0 & \text { Methoxych1or } & .00 \\ \text { DDD } & .00 & \text { Mirex } & .00 \\ \text { DDE } & .00 & \text { PCB } & .0 \\ \text { DDT } & .00 & \text { PCN } & .00 \\ \text { Dieldrin } & .00 & \text { Silvex } & 1.4 \\ \text { Endrin } & .00 & \text { Toxaphene } & 0 \\ \text { Heptachlor } & .00 & 2,4-D & .00 \\ \text { Heptachlor epoxide } & .00 & 2,4,5-T & .00\end{array}$

ANALYSIS OF SELECTED GROSS RADIOACTIVITY

(Total activity concentrations in picocuries per liter)

Gross Alpha, as

uranium natural $<2.0$

Gross Beta, as

cesium-137
Gross Beta, as

strontium -90

2.5 
PUBLIC WATER SUPPLY: Tamarac

COUNTY: Broward

COLLECTION DATE: $11-23-77$

SAMPLING POINT (1) Treated water-261127080172201, tap at house, 107th Terrace and 80 th Court.

(2) Raw water-

ANALYSIS OF MAJOR CHEMICAL CONSTITUENTS AND PHYSICAL PROPERTIES

(Dissolved concentrations in milligrams per liter, except as indicated)

(1) (2)

(1)

$\begin{array}{lc}\text { Silica ( } & 12 \\ \text { Calcium (Ca) } & 29 \\ \text { Magnesium (Mg) } & 3.4 \\ \text { Sodium (Na) } & 33 \\ \text { Potassium (K) } & 2.2 \\ \text { Bicarbonate (HCO3) } & 40 \\ \text { Carbonate (CO3) } & 0 \\ \text { Sulfate (SO4) } & 39 \\ \text { Chloride (C1) } & 68 \\ \text { Fluoride (F) } & .2 \\ \text { Nitrate (NO3-N), } & \\ \quad \text { tota1 (NO2-N), } & .05 \\ \text { Nitrite (NO2-N), } \\ \quad \text { tota1 }\end{array}$

Dissolved solids

(residue at $180^{\circ} \mathrm{C}$ ) 234

Hardness as $\mathrm{CaCO} 3$

$(\mathrm{Ca}, \mathrm{Mg}$ )

87

Noncarbonate hardness as $\mathrm{CaCO}_{3}$

54

Percent sodium 45

Alkalinity as $\mathrm{CaCO} 3 \quad 33$

Specific conductance

(umhos $/ \mathrm{cm}$ at $25^{\circ} \mathrm{C}$ )

380

$\mathrm{pH}$ (units)

7.6

Temperature $\left({ }^{\circ} \mathrm{C}\right) \quad 25.0$

Color (Pt-Co units) 15

Turbidity (NTU)

1

ANALYSIS OF SELECTED TRACE ELEMENTS

(Total concentrations in micrograms per liter, except as indicated)

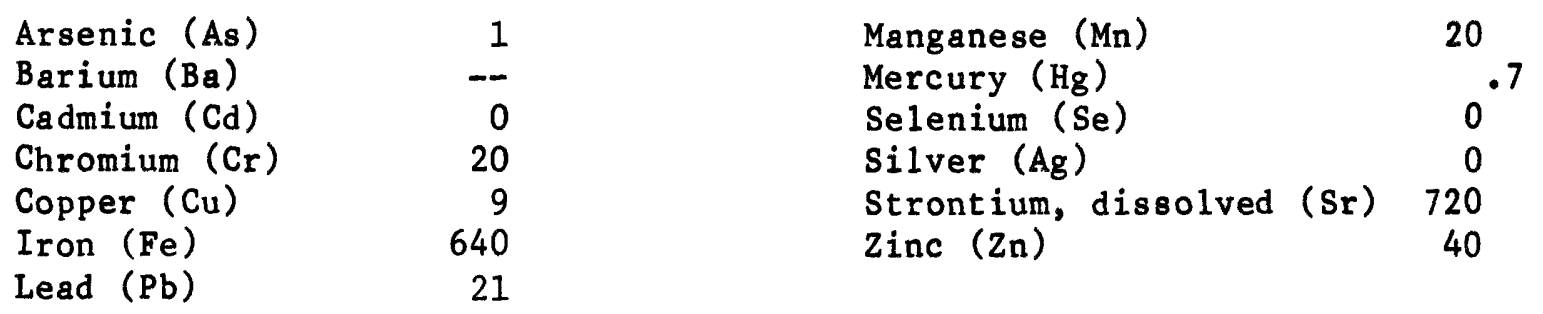

ANALYSIS OF SELECTED PESTICIDE AND INDUSTRIAL COMPOUNDS

(Total concentrations in micrograms per liter)

$\begin{array}{llll}\text { Aldrin } & .00 & \text { Lindane } & .00 \\ \text { Chlordane } & .0 & \text { Methoxych1or } & .00 \\ \text { DDD } & .00 & \text { Mirex } & .00 \\ \text { DDE } & .00 & \text { PCB } & .0 \\ \text { DDT } & .00 & \text { PCN } & .00 \\ \text { Dieldrin } & .00 & \text { Silvex } & .06 \\ \text { Endrin } & .00 & \text { Toxaphene } & .00 \\ \text { Heptach1or } & .00 & 2,4-D & .00\end{array}$

ANALYSIS OF SELECTED GROSS RADIOACTIVITY

(Total activity concentrations in picocuries per 1iter)

Gross Alpha, as uranium natural $<2.0$

Gross Beta, as

cesium-137
Gross Beta, as

strontium-90

2.5 
PUBLIC WATER SUPPLY: Tampa

COUNTY: Hillsborough

COLLECTION DATE: $12-16-77$

SAMPLING POINT (1) Treated water-280058082252809, tap in laboratory of water treatment plant.

(2) Raw water-280057082252000, tap inside old water treatment laboratory.

ANALYSIS OF MAJOR CHEMICAL CONSTITUENTS AND PHYSICAL PROPERTIES

(Dissolved concentrations in milligrams per liter, except as indicated)

(1) (2)

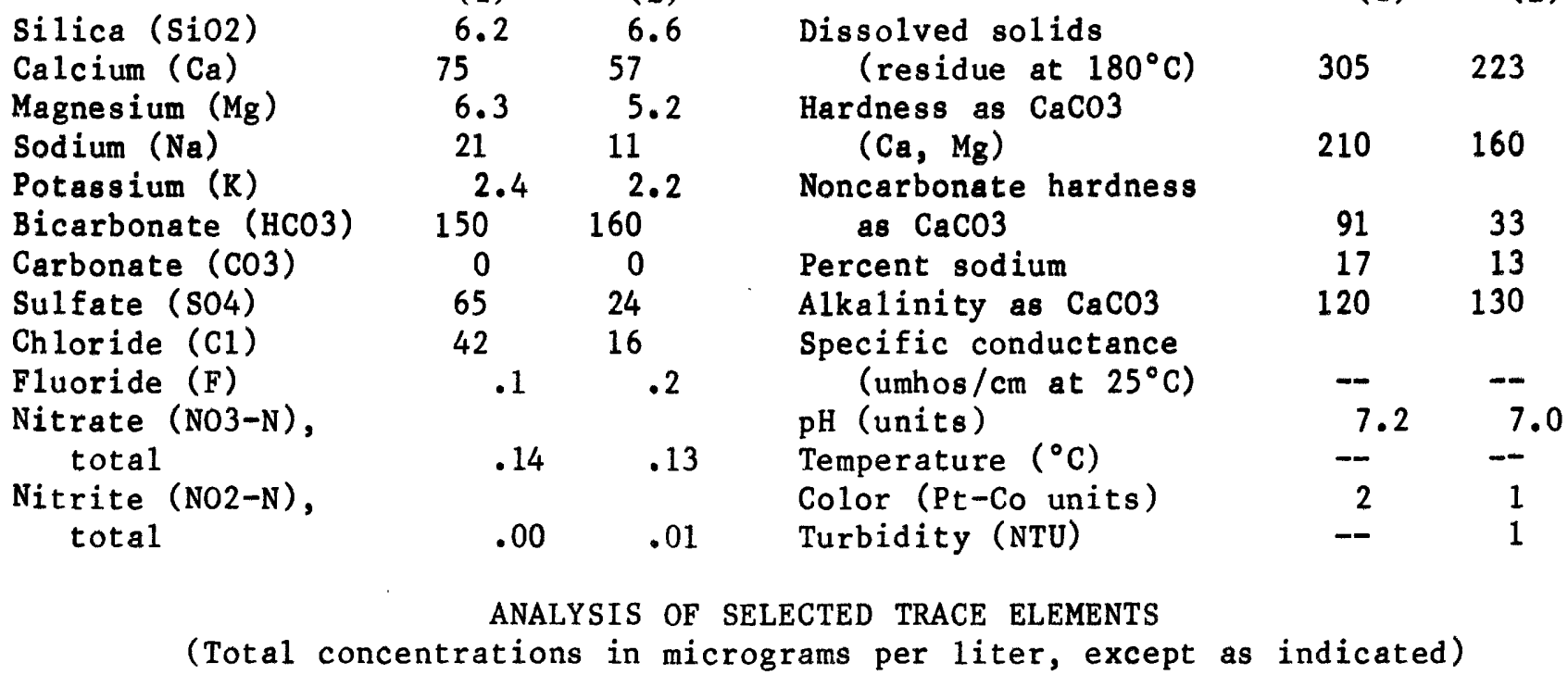

$\begin{array}{lrrlrr}\text { Arsenic (As) } & 3 & 3 & \text { Manganese (Mn) } & 0 & 10 \\ \text { Barium (Ba) } & - & -- & \text { Mercury (Hg) } & <.5 & <.5 \\ \text { Cadmium (Cd) } & 0 & 0 & \text { Selenium (Se) } & 0 & 0 \\ \text { Chromium (Cr) } & 10 & 10 & \text { Silver (Ag) } & 1 & 1 \\ \text { Copper (Cu) } & 3 & 3 & \text { Strontium, dissolved (Sr) } & 460 & 430 \\ \text { Iron (Fe) } & 20 & 60 & \text { Zinc ( } \mathrm{Cn}) & 20 & 10 \\ \text { Lead (Pb) } & 4 & 6 & & & \end{array}$

ANALYSIS OF SELECTED PESTICIDE AND INDUSTRIAL COMPOUNDS

(Total concentrations in micrograms per liter)

$\begin{array}{llllll}\text { Aldrin } & .00 & .00 & \text { Lindane } & .00 & .00 \\ \text { Chlordane } & .0 & .0 & \text { Methoxychlor } & .00 & .00 \\ \text { DDD } & .00 & .00 & \text { Mirex } & .00 & .00 \\ \text { DDE } & .00 & .00 & \text { PCB } & .0 & .0 \\ \text { DDT } & .00 & .00 & \text { PCN } & .00 & .00 \\ \text { Dieldrin } & .00 & .00 & \text { Silvex } & .00 & .00 \\ \text { Endrin } & .00 & .00 & \text { Toxaphene } & 0 & 0 \\ \text { Heptachlor } & .00 & .00 & 2,4-D & .00 & .02 \\ \text { Heptachlor epoxide } & .00 & .00 & 2,4,5-T & .00 & .00\end{array}$

ANALYSIS OF SELECTED GROSS RADIOACTIVITY

(Total activity concentrations in picocuries per liter)

Gross Alpha, as uranium natural

Grass Beta, as

cesium-137
Gross Beta, as

strontium-90 
PUBLIC WATER SUPPLY: Temple Terrace COUNTY: Hillsborough COLLECTION DATE: 12-21-77

SAMPLING POINT (1) Treated water-280243082231909, tap outside water department office on west side.

(2) Raw water-

ANALYSIS OF MAJOR CHEMICAL CONSTITUENTS AND PHYSICAL PROPERTIES (Dissolved concentrations in milligrams per liter, except as indicated)

$\begin{array}{lc}\text { Silica (SiO2) } & (1) \\ \text { Calcium (Ca) } & 12 \\ \text { Magnesium (Mg) } & 63 \\ \text { Sodium (Na) } & 4.7 \\ \text { Potassium (K) } & 5.3 \\ \text { Bicarbonate (HCO3) } & 130 \\ \text { Carbonate (CO3) } & .7 \\ \text { Sulfate (SO4) } & 58 \\ \text { Chloride (C1) } & 9.5 \\ \text { Fluoride (F) } & .1 \\ \text { Nitrate (NO3-N), } & \\ \quad \text { total (NO2-N), } & .85 \\ \text { Nitrite } & \\ \quad \text { total } & .00\end{array}$

(2)
(1)

Dissolved solids (residue at $180^{\circ} \mathrm{C}$ ) 222

Hardness as $\mathrm{CaCO} 3$ $(\mathrm{Ca}, \mathrm{Mg}$ ) 180

Noncarbonate hardness as $\mathrm{CaCO} 3$

Percent sodium

Alkalinity as $\mathrm{CaCO} 3$

Specific conductance

(umhos $/ \mathrm{cm}$ at $25^{\circ} \mathrm{C}$ )

$\mathrm{pH}$ (units)

Temperature $\left({ }^{\circ} \mathrm{C}\right)$

Color (Pt-Co units)

Turbidity (NTU)

ANALYSIS OF SELECTED TRACE ELEMENTS

(Total concentrations in micrograms per liter, except as indicated)

$\begin{array}{lrlc}\text { Arsenic (As) } & 2 & \text { Manganese (Mn) } & 0 \\ \text { Barium (Ba) } & -- & \text { Mercury (Hg) } & <.5 \\ \text { Cadmium (Cd) } & 0 & \text { Selenium (Se) } & 1 \\ \text { Chromium (Cr) } & 30 & \text { Silver (Ag) } & 1 \\ \text { Copper (Cu) } & 6 & \text { Strontium, dissolved ( } \mathrm{Sr}) & 590 \\ \text { Iron (Fe) } & 170 & \text { Zinc ( } \mathrm{Gn} \text { ) } & 40 \\ \text { Lead (Pb) } & 4 & & \end{array}$

ANALYSIS OF SELECTED PESTICIDE AND INDUSTRIAL COMPOUNDS

(Total concentrations in micrograms per liter)

$\begin{array}{llll}\text { Aldrin } & .00 & \text { Lindane } & .00 \\ \text { Chlordane } & .0 & \text { Methoxychlor } & .00 \\ \text { DDD } & .00 & \text { Mirex } & .00 \\ \text { DDE } & .00 & \text { PCB } & .0 \\ \text { DDT } & .00 & \text { PCN } & .00 \\ \text { Dieldrin } & .00 & \text { Silvex } & .00 \\ \text { Endrin } & .00 & \text { Toxaphene } & 0 \\ \text { Heptachlor } & .00 & 2,4-D & .00 \\ \text { Heptachlor epoxide } & .00 & 2,4,5-T & .00\end{array}$

ANALYSIS OF SELECTED GROSS RADIOACTIVITY

(Total activity concentrations in picocuries per liter)

Gross Alpha, as
$\begin{aligned} & \text { uranium natural } \\ & \text { Gross Beta, as } \\ & \text { cesium-137 }\end{aligned}$

Gross Beta, as

strontium-90 
PUBLIC WATER SUPPLY: Umatilla

COUNTY: Lake

COLLECTION DATE: 11-03-77

SAMPLING POINT (1) Treated water-285627081400001, tap inside water treatment plant.

(2) Raw water-

ANALYSIS OF MAJOR CHEMICAL CONSTITUENTS AND PHYSICAL PROPERTIES (Dissolved concentrations in milligrams per liter, except as indicated)

(1) (2)

$\begin{array}{lc}\text { Silica (SiO2) } & 14 \\ \text { Calcium (Ca) } & 31 \\ \text { Magnesium (Mg) } & 6.5 \\ \text { Sodium (Na) } & 6.7 \\ \text { Potassium (K) } & 1.2 \\ \text { Bicarbonate (HCO3) } & 120 \\ \text { Carbonate (CO3) } & 0 \\ \text { Sulfate (SO4) } & 2.6 \\ \text { Chloride (C1) } & 17 \\ \text { Fluoride (F) } & .1 \\ \text { Nitrate (NO3-N), } & \\ \quad \text { total (NO2-N), } & .01 \\ \text { Nitrite (NO2-N) } & \\ \quad \text { total } & .00\end{array}$

Dissolved solids

$$
\text { (residue at } 180^{\circ} \mathrm{C} \text { ) } \quad 127
$$

Hardness as $\mathrm{CaCO} 3$

$$
(\mathrm{Ca}, \mathrm{Mg}) \quad 100
$$

Noncarbonate hardness as $\mathrm{CaCO} 3$

Percent sodium

Alkalinity as $\mathrm{CaCO} 3$

Specific conductance

(umhos $/ \mathrm{cm}$ at $25^{\circ} \mathrm{C}$ )

$\mathrm{pH}$ (units)

Temperature $\left({ }^{\circ} \mathrm{C}\right)$

Color (Pt-Co units)

Turbidity (NTU)
(1)

ANALYSIS OF SELECTED TRACE ELEMENTS

(Total concentrations in micrograms per liter, except as indicated)

$\begin{array}{lrlc}\text { Arsenic (As) } & 0 & \text { Manganese (Mn) } & 10 \\ \text { Barium (Ba) } & 0 & \text { Mercury (Hg) } & <.5 \\ \text { Cadmium (Cd) } & 1 & \text { Selenium (Se) } & 0 \\ \text { Chromium (Cr) } & 10 & \text { Silver (Ag) } & 0 \\ \text { Copper (Cu) } & 130 & \text { Strontium, dissolved (Sr) } & 70 \\ \text { Iron (Fe) } & 100 & \text { Zinc (Zn) } & 10 \\ \text { Lead (Pb) } & 10 & & \end{array}$

ANALYSIS OF SELECTED PESTICIDE AND INDUSTRIAL COMPOUNDS

(Total concentrations in micrograms per liter)

$\begin{array}{lllc}\text { Aldrin } & .00 & \text { Lindane } & .00 \\ \text { Chlordane } & .0 & \text { Methoxychlor } & .00 \\ \text { DDD } & .00 & \text { Mirex } & .00 \\ \text { DDE } & .00 & \text { PCB } & .0 \\ \text { DDT } & .00 & \text { PCN } & .00 \\ \text { Dieldrin } & .00 & \text { Silvex } & 0 \\ \text { Endrin } & .00 & \text { Toxaphene } & - \\ \text { Heptachlor } & .00 & 2,4-D & -- \\ \text { Heptachlor epoxide } & .00 & 2,4,5-\mathrm{T} & \end{array}$

ANALYSIS OF SELECTED GROSS RADIOACTIVITY

(Total activity concentrations in picocuries per liter)

Gross Alpha, as

uranium natura1 --

Gross Beta, as

cesium-137
Gross Beta, as

strontium-90 
PUBLIC WATER SUPPLY: Union Correctional Institute

COUNTY: Union

COLLECTION DATE: 12-09-77

SAMPLING POINT (1) Treated water-30034208111390, tap in sink inside water treatment plant northwest of correctional facility.

(2) Raw water-

ANALYSIS OF MAJOR CHEMICAL CONSTITUENTS AND PHYSICAL PROPERTIES (Dissolved concentrations in milligrams per liter, except as indicated)

(1) (2)

$\begin{array}{lc}\text { Silica (SiO2) } & 18 \\ \text { Calcium (Ca) } & 35 \\ \text { Magnesium (Mg) } & 13 \\ \text { Sodium (Na) } & 7.4 \\ \text { Potassium (K) } & 1.1 \\ \text { Bicarbonate (HCO3) } & 150 \\ \text { Carbonate (CO3) } & 0 \\ \text { Sulfate (SO4) } & 18 \\ \text { Chloride (C1) } & 8.2 \\ \text { Fluoride (F) } & .3 \\ \text { Nitrate (NO3-N), } & \\ \quad \text { total (NO2-N), } & .01 \\ \text { Nitrite (NO2) } \\ \quad \text { total }\end{array}$

(1)

Dissolved solids (residue at $180^{\circ} \mathrm{C}$ )

187

Hardness as $\mathrm{CaCO} 3$ $(\mathrm{Ca}, \mathrm{Mg}$ )

140

Noncarbonate hardness as $\mathrm{CaCO} 3$

Percent sodium

Alkalinity as $\mathrm{CaCO} 3$

Specific conductance

120

.2

(umhos $/ \mathrm{cm}$ at $25^{\circ} \mathrm{C}$ )

280

$\mathrm{pH}$ (units)

Temperature $\left({ }^{\circ} \mathrm{C}\right)$

24.0

Color (Pt-Co units)

0

Turbidity (NTU)

1

ANALYSIS OF SELECTED TRACE ELEMENTS

(Total concentrations in micrograms per liter, except as indicated)

$\begin{array}{lrlr}\text { Arsenic (As) } & 0 & \text { Manganese (Mn) } & 0 \\ \text { Barium (Ba) } & 0 & \text { Mercury (Hg) } & <.5 \\ \text { Cadmium (Cd) } & 0 & \text { Selenium ( } \mathrm{Se}) & 0 \\ \text { Chromium (Cr) } & <10 & \text { Silver (Ag) } & 0 \\ \text { Copper (Cu) } & 39 & \text { Strontium, dissolved (Sr) } & 730 \\ \text { Iron (Fe) } & 0 & \text { Zinc ( } n \text { ) } & 0 \\ \text { Lead (Pb) } & 6 & & \end{array}$

ANALYSIS OF SELECTED PESTICIDE AND INDUSTRIAL COMPOUNDS (Total concentrations in micrograms per liter)

$\begin{array}{llll}\text { Aldrin } & .00 & \text { Lindane } & .00 \\ \text { Ch1ordane } & .0 & \text { Methoxych1or } & .00 \\ \text { DDD } & .00 & \text { Mirex } & .00 \\ \text { DDE } & .00 & \text { PCB } & .0 \\ \text { DDT } & .00 & \text { PCN } & .00 \\ \text { Dieldrin } & .00 & \text { Silvex } & .00 \\ \text { Endrin } & .00 & \text { Toxaphene } & 0 \\ \text { Heptach1or } & .00 & 2,4-D & .00 \\ \text { Heptach1or epoxide } & .00 & 2,4,5-T & .00\end{array}$

ANALYSIS OF SELECTED GROSS RADIOACTIVITY

(Total activity concentrations in picocuries per liter)

Gross Alpha, as uranium natural $<2.9$

Gross Beta, as

cesium-137
Gross Beta, as

strontium-90 
PUBLIC WATER SUPPLY: Venice

COUNTY: Sarasota COLLECTION DATE: 12-21-77

SAMPLING POINT (1) Treated water-270601082261409, composite, tap outside old water treatment plant.

(2) Raw water-

ANALYSIS OF MAJOR CHEMICAL CONSTITUENTS AND PHYSICAL PROPERTIES (Dissolved concentrations in milligrams per liter, except as indicated)

Silica (SiO2)

(1)

Calcium $(\mathrm{Ca})$

Magnesium (Mg)

Sodium ( $\mathrm{Na})$

Potassium (K)

Bicarbonate ( $\mathrm{HCO} 3$ )

Carbonate ( $\mathrm{CO} 3)$

Sulfate (S04)

Chloride (C1)

Fluoride (F)

Nitrate (NO3-N), total

Nitrite (NO2-N), total
(2)

Dipsolved solids

(residue at $180^{\circ} \mathrm{C}$ )

Hardness as $\mathrm{CaCO} 3$

( $\mathrm{Ca}, \mathrm{Mg}$ )

Noncarbonate hardness

as $\mathrm{CaCO} 3$

Percent sodium

Alkalinity as $\mathrm{CaCO} 3$

Specific conductance

(umhos $/ \mathrm{cm}$ at $25^{\circ} \mathrm{C}$ )

pH (units)

Temperature $\left({ }^{\circ} \mathrm{C}\right)$

Color (Pt-Co units)

Turbidity (NTU)

(1)

ANALYSIS OF SELECTED TRACE ELEMENTS

(Total concentrations in micrograms per liter, except as indicated)

Arsenic (As)

Barium ( $\mathrm{Ba})$

Cadmium (Cd)

Chromium ( $\mathrm{Cr}$ )

Copper $(\mathrm{Cu})$

Iron ( $\mathrm{Fe}$ )

Lead $(\mathrm{Pb})$
Mangane se $(\mathrm{Mn})$

Mercury ( $\mathrm{Hg}$ )

Selenium ( $\mathrm{Se}$ )

Silver $(\mathrm{Ag})$

Strontium, dissolved $(\mathrm{Sr})$

Zinc $(\mathrm{Zn})$

ANALYSIS OF SELECTED PESTICIDE AND INDUSTRIAL COMPOUNDS

(Total concentrations in micrograms per liter)
Aldrin
Chlordane
DDD
DDE
DDT
Dieldrin
Endrin
Heptach 1 or
Heptachlor epoxide

Lindane

Methoxych lor

Mirex

PCB

PCN

Silvex

Toxaphene

$2,4-D$

$2,4,5-T$

ANALYSIS OF SELECTED GROSS RADIOACTIVITY

(Total activity concentrations in picocuries per liter)

Gross A1pha, as uranium natural 12

Gross Beta, as

cesium-137
Gross Beta, as

strontium-90

4.7 
PUBLIC WATER SUPPLY: Venice Gardens

COUNTY: Sarasota

COLLECTION DATE: 12-21-77

SAMPLING POINT (1) Treated water-270520082260409, tap inside water treatment plant number 2.

(2) Raw water-

ANALYSIS OF MAJOR CHEMICAL CONSTITUENTS AND PHYSICAL PROPERTIES (Dissolved concentrations in milligrams per liter, except as indicated)

(1)

$\begin{array}{lc}\text { Silica (SiO2) } & 27 \\ \text { Calcium (Ca) } & 120 \\ \text { Magnesium (Mg) } & 44 \\ \text { Sodium (Na) } & 60 \\ \text { Potassium (K) } & 3.6 \\ \text { Bicarbonate (HCO3) } & 240 \\ \text { Carbonate (CO3) } & 0 \\ \text { Sulfate (SO4) } & 230 \\ \text { Chloride (C1) } & 140 \\ \text { Fluoride (F) } & .8 \\ \text { Nitrate (NO3-N), } & \\ \quad \text { total (NO2-N), } & .02 \\ \text { Nitrite (NO2-N) } & \\ \quad \text { total } & .00\end{array}$

(2)

Dissolved solids

$$
\text { (residue at } 180^{\circ} \mathrm{C} \text { ) } 800
$$

Hardness as $\mathrm{CaCO} 3$

$(\mathrm{Ca}, \mathrm{Mg})$

480

Noncarbonate hardness as $\mathrm{CaCO} 3$

Percent sodium

290

Alkalinity as $\mathrm{CaCO} 3$

Specific conductance

(umhos $/ \mathrm{cm}$ at $25^{\circ} \mathrm{C}$ )

$\mathrm{pH}$ (units)

Temperature $\left({ }^{\circ} \mathrm{C}\right)$

Color (Pt-Co units)

Turbidity (NTU)
(1)

ANALYSIS OF SELECTED TRACE ELEMENTS

(Total concentrations in micrograms per liter, except as indicated)

$\begin{array}{lrlr}\text { Arsenic (As) } & 1 & \text { Manganese (Mn) } & 10 \\ \text { Barium (Ba) } & -- & \text { Mercury (Hg) } & <.5 \\ \text { Cadmium (Cd) } & 0 & \text { Selenium (Se) } & 0 \\ \text { Chromium (Cr) } & 10 & \text { Silver (Ag) } & 0 \\ \text { Copper (Cu) } & 67 & \text { Strontium, dissolved (Sr) } & 2900 \\ \text { Iron (Fe) } & 210 & \text { Zinc ( } \mathrm{Cn}) & 40 \\ \text { Lead (Pb) } & 3 & & \end{array}$

ANALYSIS OF SELECTED PESTICIDE AND INDUSTRIAL COMPOUNDS

(Total concentrations in micrograms per 1iter)

$\begin{array}{llll}\text { Aldrin } & .00 & \text { Lindane } & .00 \\ \text { Chlordane } & .0 & \text { Methoxychlor } & .00 \\ \text { DDD } & .00 & \text { Mirex } & .00 \\ \text { DDE } & .00 & \text { PCB } & .0 \\ \text { DDT } & .00 & \text { PCN } & .00 \\ \text { Dieldrin } & .00 & \text { Silvex } & .00 \\ \text { Endrin } & .00 & \text { Toxaphene } & 0 \\ \text { Heptachlor } & .00 & 2,4-D & .02 \\ \text { Heptachlor epoxide } & .00 & 2,4,5-T & .00\end{array}$

ANALYSIS OF SELECTED GROSS RADIOACTIVITY

(Total activity concentrations in picocuries per liter)

Gross Alpha, as uranium natural 33

Gross Beta, as

cesium-137
Gross Beta, as

strontium-90

12 
PUBLIC WATER SUPPLY: Vernon COUNTY: Washington

SAMPLING POINT (1) Treated water-303739085424390, tap on east side of commons in town square across from City Hall.

(2) Raw water-

ANALYSIS OF MAJOR CHEMICAL CONSTITUENTS AND PHYSICAL PROPERTIES (Dissolved concentrations in milligrams per 1 iter, except as indicated)

(1) (2)

9.2

42

7.0

2.8

.5

160

0

2.3

3.7

.1

.16

.00
Dissolved solids

(residue at $180^{\circ} \mathrm{C}$ ) $\quad 124$

Hardness as $\mathrm{CaCO} 3$

$(\mathrm{Ca}, \mathrm{Mg}$ )

Noncarbonate hardness as $\mathrm{CaCO} 3$

Percent sodium

Alkalinity as $\mathrm{CaCO} 3$

Specific conductance

(umhos $/ \mathrm{cm}$ at $25^{\circ} \mathrm{C}$ )

pH (units)

Temperature $\left({ }^{\circ} \mathrm{C}\right)$

Color (Pt-Co units)

Turbidity (NTU)

1
(1)

ANALYSIS OF SELECTED TRACE ELEMENTS

(Total concentrations in micrograms per liter, except as indicated)

Arsenic (As)

Barium ( $\mathrm{Ba}$ )

Cadmium (Cd)

Chromium ( $\mathrm{Cr}$ )

Copper ( $\mathrm{Cu})$

Iron ( $\mathrm{Fe}$ )

Lead $(\mathrm{Pb})$

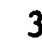

3
-0
30
4
90
28
Manganese (Mn)

Mercury ( $\mathrm{Hg}$ )

Selenium ( $\mathrm{Se}$ )

Silver ( $\mathrm{Ag})$

Strontium, dissolved ( $\mathrm{Sr}$ ) 100

Zinc $(\mathrm{z} n)$
10

$<.5$

0

0

ANALYSIS OF SELECTED PESTICIDE AND INDUSTRIAL COMPOUNDS

(Total concentrations in micrograms per liter)

Aldrin

Chlordane

DDD

$\mathrm{DDE}$

DDT

Dieldrin

Endrin

Heptachlor

Heptachlor epoxide
.00

.0

.00

.00

.00

.00

.00

.00

.00
Lindane

Methoxych 1or

Mirex

PCB

PCN

Silvex

Toxaphene

2,4-D

$2,4,5-T$
.00

.00

.00

.0

.00

.00

0

.00

.00

ANALYSIS OF SELECTED GROSS RADIOACTIVITY

(Total activity concentrations in picocuries per liter)

Gross Alpha, as

uranium natural $<1.6$

Gross Beta, as

cesium-137
Gross Beta, as

strontium-90 
PUBLIC WATER SUPPLY: Wahneta

COUNTY: Polk

COLLECTION DATE: $12-14-77$

SAMPLING POINT (1) Treated water -275726081433509 , tap outside on south side of water treatment plant.

(2) Raw water-

ANALYSIS OF MAJOR CHEMICAL CONSTITUENTS AND PHYSICAL PROPERTIES

(Dissolved concentrations in milligrams per liter, except as indicated)

(1) (2)

$\begin{array}{lc}\text { Silica (SiO2) } & 19 \\ \text { Calcium (Ca) } & 38 \\ \text { Magnesium (Mg) } & 8.0 \\ \text { Sodium (Na) } & 10 \\ \text { Potassium (K) } & 1.4 \\ \text { Bicarbonate (HCO3) } & 150 \\ \text { Carbonate (CO3) } & 0 \\ \text { Sulfate (SO4) } & 2.1 \\ \text { Chloride (C1) } & 19 \\ \text { Fluoride (F) } & .2 \\ \text { Nitrate (NO3-N), } & \\ \quad \text { total (NO2-N), } & .01 \\ \text { Nitrite (N02) } \\ \quad \text { total }\end{array}$

Dissolved solids

(1)

182

Hardness as $\mathrm{CaCO} 3$

$(\mathrm{Ca}, \mathrm{Mg})$

130

Noncarbonate hardness as $\mathrm{CaCO} 3$

Percent sodium

Alkalinity as $\mathrm{CaCO} 3$

14

Specific conductance

(umhos $/ \mathrm{cm}$ at $25^{\circ} \mathrm{C}$ )

$\mathrm{pH}$ (units)

Temperature $\left({ }^{\circ} \mathrm{C}\right)$

Color (Pt-Co units)

Turbidity (NTU)

120

ANALYSIS OF SELECTED TRACE ELEMENTS

(Total concentrations in micrograms per liter, except as indicated)

$\begin{array}{lr}\text { Arsenic (As) } & 0 \\ \text { Barium (Ba) } & -- \\ \text { Cadmium (Cd) } & 0 \\ \text { Chromium (Cr) } & <10 \\ \text { Copper (Cu) } & 17 \\ \text { Iron (Fe) } & 50 \\ \text { Lead (Pb) } & 6\end{array}$

Manganese $(\mathrm{Mn})$

Mercury ( $\mathrm{Hg}$ )

0

Selenium (Se)

$<.5$

0

Silver (Ag)

0

$\begin{array}{ll}17 & \text { Strontium, dissolved (Sr) } 150 \\ \text { Zinc (Zn) } & 90\end{array}$

50

$\begin{array}{ll}17 & \text { Strontium, dissolved (Sr) } 150 \\ \text { Zinc (Zn) } & 90\end{array}$

0

ANALYSIS OF SELECTED PESTICIDE AND INDUSTRIAL COMPOUNDS

(Total concentrations in micrograms per 1iter)

$\begin{array}{llll}\text { Aldrin } & .00 & \text { Lindane } & .00 \\ \text { Chlordane } & .0 & \text { Methoxychlor } & .00 \\ \text { DDD } & .00 & \text { Mirex } & .00 \\ \text { DDE } & .00 & \text { PCB } & .0 \\ \text { DDT } & .00 & \text { PCN } & .00 \\ \text { Dieldrin } & .00 & \text { Silvex } & .00 \\ \text { Endrin } & .00 & \text { Toxaphene } & 0 \\ \text { Heptachlor } & .00 & 2,4-D & .00 \\ \text { Heptachlor epoxide } & .00 & 2,4,5-T & .00\end{array}$

ANALYSIS OF SELECTED GROSS RADIOACTIVITY

(Total activity concentrations in picocuries per liter)

Gross Alpha, as uranium natural $\quad 4.6$

Gross Beta, as

cesium-137
Gross Beta, as

strontium-90 
PUBLIC WATER SUPPLY: Walt Disney World COUNTY: Orange COLLECTION DATE: 11-02-77

SAMPLING POINT (1) Treated water-282530081344501, (composite we11s 8, 9, \& 10), tap outside pump housing.

(2) Raw water-

ANALYSIS OF MAJOR CHEMICAL CONSTITUENTS AND PHYSICAL PROPERTIES (Dissolved concentrations in milligrams per liter, except as indicated)

(1) (2)

9.2

25

5.4

2.6

Sodium $(\mathrm{Na})$

Potassium (K)

Bicarbonate ( $\mathrm{HCO} 3$ )

Carbonate ( $\mathrm{CO} 3$ )

Sulfate ( 04 )

Chloride (C1)

Fluoride (F)

Nitrate (NO3-N), total

Nitrite (NO2-N),

total
(1)

Dissolved solids

(residue at $180^{\circ} \mathrm{C}$ )

Hardness as $\mathrm{CaCO} 3$

( $\mathrm{Ca}, \mathrm{Mg}$ )

96

Noncarbonate hardness as $\mathrm{CaCO} 3$

Percent sodium

Alkalinity as $\mathrm{CaCO} 3$

Specific conductance

(umhos $/ \mathrm{cm}$ at $25^{\circ} \mathrm{C}$ )

$\mathrm{pH}$ (units)

Temperature $\left({ }^{\circ} \mathrm{C}\right)$

Color (Pt-Co units)

Turbidity (NTU)

ANALYSIS OF SELECTED TRACE ELEMENTS

(Total concentrations in micrograms per liter, except as indicated)

$\begin{array}{lr}\text { Arsenic (As) } & 0 \\ \text { Barium (Ba) } & 0 \\ \text { Cadmium ( } \mathrm{Cd} \text { ) } & 0 \\ \text { Chromium (Cr) } & 10 \\ \text { Copper (Cu) } & 3 \\ \text { Iron (Fe) } & 20 \\ \text { Lead ( } \mathrm{Pb}) & 7\end{array}$

$\begin{array}{lc}\text { Manganese (Mn) } & 0 \\ \text { Mercury ( } \mathrm{Hg}) & <.5 \\ \text { Selenium ( Se) } & 0 \\ \text { Silver (Ag) } & 0 \\ \text { Strontium, dissolved ( } \mathrm{Sr}) & 100 \\ \text { Zinc (Zn) } & 20\end{array}$

ANALYSIS OF SELECTED PESTICIDE AND INDUSTRIAL COMPOUNDS

(Total concentrations in micrograms per liter)

$\begin{array}{llll}\text { Aldrin } & .00 & \text { Lindane } & .00 \\ \text { Chlordane } & .0 & \text { Methoxychlor } & .00 \\ \text { DDD } & .00 & \text { Mirex } & .00 \\ \text { DDE } & .00 & \text { PCB } & .0 \\ \text { DDT } & .00 & \text { PCN } & .00 \\ \text { Dieldrin } & .00 & \text { Silvex } & .00 \\ \text { Endrin } & .00 & \text { Toxaphene } & 0 \\ \text { Heptachlor } & .00 & 2,4-D & .00 \\ \text { Heptachlor epoxide } & .00 & 2,4,5-\mathrm{T} & .00\end{array}$

ANALYSIS OF SELECTED GROSS RADIOACTIVITY

(Total activity concentrations in picocuries per liter)

Gross Alpha, as

uranium natural --

Gross Beta, as

cesium-137
Gross Beta, as

strontium -90 
PUBLIC WATER SUPPLY: Weeki Wachee

COUNTY: Hernando

COLLECTION DATE: 12-19-77

SAMPLING POINT (1) Treated water-283206082365809, tap inside west end of main building.

(2) Raw water-

ANALYSIS OF MAJOR CHEMICAL CONSTITUENTS AND PHYSICAL PROPERTIES

(Dissolved concentrations in milligrams per liter, except as indicated)

(1) (2)

(1)

$\begin{array}{lc}\text { Silica ( } & 9.6 \\ \text { Calcium (Ca) } & 39 \\ \text { Magnesium (Mg) } & 1.9 \\ \text { Sodium (Na) } & 3.2 \\ \text { Potassium (K) } & .4 \\ \text { Bicarbonate (HCO3) } & 120 \\ \text { Carbonate (CO3) } & 0 \\ \text { Sulfate (SO4) } & 1.5 \\ \text { Chloride (C1) } & 7.1 \\ \text { Fluoride (F) } & .0 \\ \text { Nitrate (NO3-N), } & \\ \quad \text { total (NO2-N), } & .21 \\ \text { Nitrite } \quad & \\ \quad \text { total }\end{array}$

Dissolved solids

(residue at $180^{\circ} \mathrm{C}$ ) $\quad 143$

Hardness as $\mathrm{CaCO} 3$

( $\mathrm{Ca}, \mathrm{Mg})$

110

Noncarbonate hardness

as $\mathrm{CaCO} 3$

Percent sodium

Alkalinity as $\mathrm{CaCO} 3$

Specific conductance

(umhos $/ \mathrm{cm}$ at $25^{\circ} \mathrm{C}$ )

pH (units)

Temperature $\left({ }^{\circ} \mathrm{C}\right)$

Color (Pt-Co units)

Turbidity (NTU)

ANALYSIS OF SELECTED TRACE ELEMENTS

(Total concentrations in micrograms per liter, except as indicated)

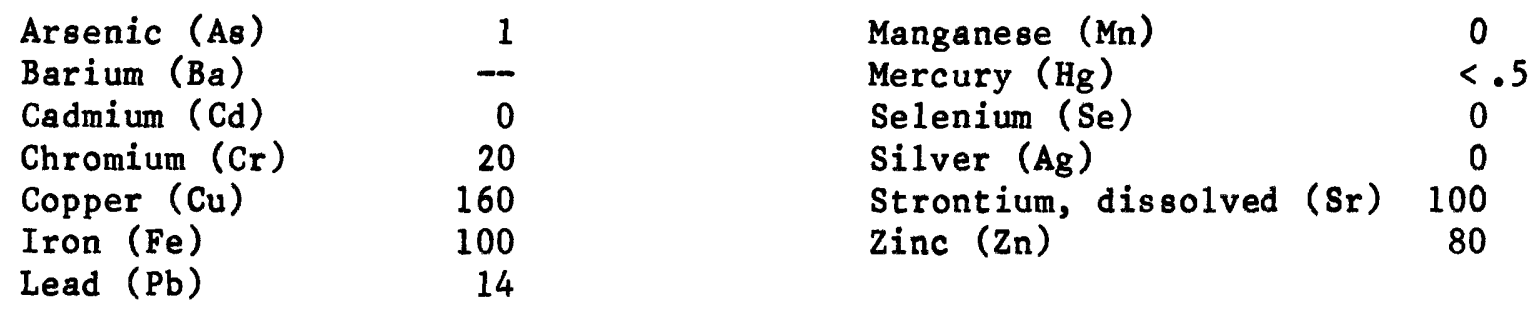

ANALYSIS OF SELECTED PESTICIDE AND INDUSTRIAL COMPOUNDS

(Total concentrations in micrograms per liter)

$\begin{array}{llll}\text { Aldrin } & .00 & \text { Lindane } & .00 \\ \text { Chlordane } & .0 & \text { Methoxych10r } & .00 \\ \text { DDD } & .00 & \text { Mirex } & .00 \\ \text { DDE } & .00 & \text { PCB } & .0 \\ \text { DDT } & .00 & \text { PCN } & .00 \\ \text { Dieldrin } & .00 & \text { Silvex } & .00 \\ \text { Endrin } & .00 & \text { Toxaphene } & 0 \\ \text { Heptachlor } & .00 & 2,4-D & .00 \\ \text { Heptachlor epoxide } & .00 & 2,4,5-T & .00\end{array}$

ANALYSIS OF SELECTED GROSS RADIOACTIVITY

(Total activity concentrations in picocuries per liter)

Gross Alpha, as

uranium natural --

Gross Beta, as

cesium-137
Gross Beta, as

strontium -90 
PUBLIC WATER SUPPLY: West Palm Beach

COUNTY: Palm Beach

COLLECTION DATE: 11-28-77

SAMPLING POINT (1) Treated water-264255080035002, tap inside water treatment plant.

(2) Raw water-264255080035001, water treatment plant Intake from Clear Lake.

ANALYSIS OF MAJOR CHEMICAL CONSTITUENTS AND PHYSICAL PROPERTIES

(Dissolved concentrations in milligrams per liter, except as indicated)

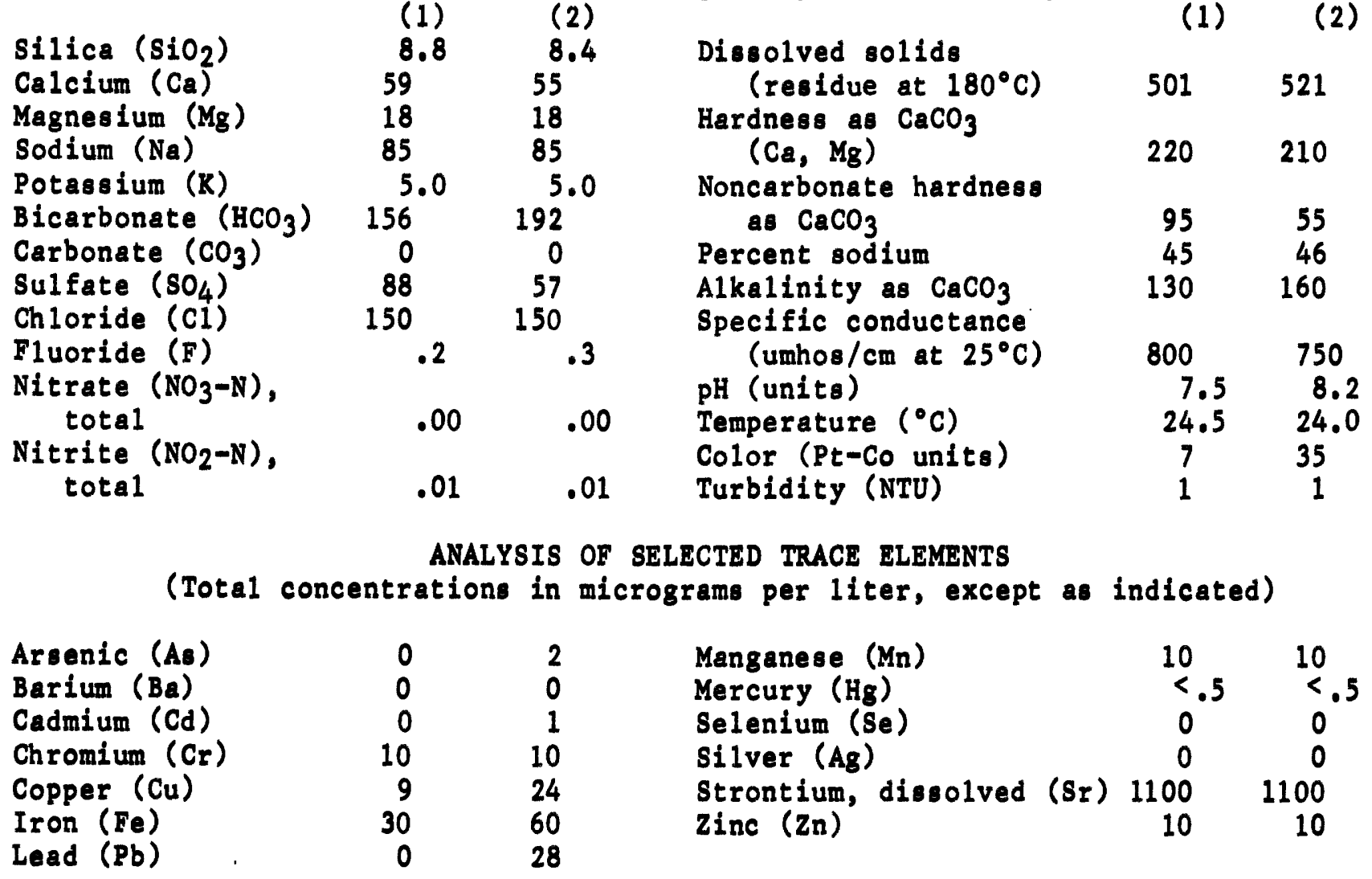

ANALYSIS OF SELECTED PESTICIDE AND INDUSTRIAL COMPOUNDS

(Total concentrations in micrograms per liter)

$\begin{array}{llllll}\text { Aldrin } & .00 & .00 & \text { Lindane } & .00 & .00 \\ \text { Chlordane } & .0 & .0 & \text { Methoxychlor } & .00 & .00 \\ \text { DDD } & .00 & .00 & \text { Mirex } & .00 & .00 \\ \text { DDE } & .00 & .00 & \text { PCB } & .0 & .0 \\ \text { DDT } & .00 & .00 & \text { PCN } & .00 & .00 \\ \text { Dieldrin } & .00 & .00 & \text { Silvex } & .03 & .01 \\ \text { Endrin } & .00 & .00 & \text { Toxaphene } & 0 & 0 \\ \text { Heptachlor } & .00 & .00 & 2,4-D & .15 & .16 \\ \text { Heptachlor epoxide } & .00 & .00 & 2,4,5-T & .00 & .00\end{array}$

ANALYSIS OF SELECTED GROSS RADIOACTIVITY

(Total activity concentrations in picocuries per liter)

Gross Alpha, as uranium natural

Gross Beta, as

cesium-137
$<1.6<5.0$

$2.6 \quad 9.1$
Gross Beta, as

strontium -90

2.3

8.1 
PUBLIC WATER SUPPLY: Wewahitchka

COUNTY: GUIf

COLLECTION DATE: 12-23-77

SAMPLING POINT (1) Treated water-300642085115090, tap outside on east end of City Hall building.

(2) Raw water-

ANALYSIS OF MAJOR CHEMICAL CONSTITUENTS AND PHYSICAL PROPERTIES (Dissolved concentrations in m $11 i$ grams per liter, except as indicated)

Silica (SiO2)

Calcium ( $\mathrm{Ca}$ )

Magnesium ( $\mathrm{Mg}$ )

Sodium (Na)

Potassium ( $\mathrm{K}$ )

Bicarbonate ( $\mathrm{HCO} 3$ )

Carbonate ( $\mathrm{CO} 3$ )

Sulfate ( $\mathrm{SO} 4$ )

Chloride (C1)

Fluoride (F)

Nitrate (NO3-N), total

Nitrite $(\mathrm{NO2}-\mathrm{N})$, total

(2)

(1)

$$
\begin{aligned}
& \text { Dissolved solids } \\
& \text { (residue at } 180^{\circ} \mathrm{C} \text { ) } \\
& \text { Hardness as CaCO3 } \\
& \text { (Ca, Mg) } \\
& \text { Noncarbonate hardness } \\
& \text { as CaCO3 } \\
& \text { Percent sodium } \\
& \text { Alkalinity as CaCO3 } \\
& \text { Specific conductance } \\
& \text { (umhos/cm at } 25^{\circ} \mathrm{C} \text { ) } \\
& \text { pH (units) } \\
& \text { Temperature ( }{ }^{\circ} \mathrm{C} \text { ) } \\
& \text { Color (Pt-Co units) } \\
& \text { Turbidity (NTU) }
\end{aligned}
$$

(1)

ANALYSIS OF SELECTED TRACE ELEMENTS

(Total concentrations in micrograms per liter, except as indicated)

Arsenic (As)

Barium ( $\mathrm{Ba}$ )

Cadmium (Cd)

Chromium (Cr)

Copper $(\mathrm{Cu})$

Iron ( $\mathrm{Fe}$ )

Lead $(\mathrm{Pb})$
Manganese ( $M n)$

Mercury ( $\mathrm{Hg}$ )

Selenium ( $\mathrm{Se}$ )

Silver ( $\mathrm{Ag}$ )

Strontium, dissolved ( $\mathrm{Sr}$ )

Zinc $(Z n)$

ANALYSIS OF SELECTED PESTICIDE AND INDUSTRIAL COMPOUNDS

(Total concentrations in micrograms per liter)

Aldrin
Chlordane
DDD
DDE
DDT
Dieldrin
Endrin
Heptachlor
Heptachlor epoxide

Lindane

Methoxych lor

Mirex

PCB

PCN

Silvex

Toxaphene

2,4-D

$2,4,5-T$

ANALYSIS OF SELECTED GROSS RADIOACTIVITY

(Total activity concentrations in picocuries per liter)

Gross Alpha, as

uranium natural $<6.0$

Gross Beta, as

cesium-137
Gross Beta, as

strontium -90
660

8.0

14.0 
PUBLIC WATER SUPPLY: White Springs

COUNTY: Hamilton

COLLECTION DATE: 12-08-77

SAMPLING POINT (1) Treated water-301945082443790, tap in rear of U.S. Post Office building (south side).

(2) Raw water-

ANALYSIS OF MAJOR CHEMICAL CONSTITUENTS AND PHYSICAL PROPERTIES (Dissolved concentrations in milligrams per liter, except as indicated)
(1)
(2)
(1)

$\begin{array}{lc}\text { Silica (SiO2) } & 27 \\ \text { Calcium (Ca) } & 45 \\ \text { Magnesium (Mg) } & 19 \\ \text { Sodium (Na) } & 5.1 \\ \text { Potassium (K) } & .7 \\ \text { Bicarbonate (HCO3) } & 210 \\ \text { Carbonate (CO3) } & 0 \\ \text { Sulfate (SO4) } & 7.3 \\ \text { Chloride (C1) } & 11 \\ \text { Fluoride (F) } & .3 \\ \text { Nitrate (NO3-N), } & \\ \text { total (NO2-N), } & .05 \\ \text { Nitrite (NO2-N) } & \\ \quad \text { total } & .00\end{array}$

Dissolved solids

(residue at $180^{\circ} \mathrm{C}$ ) 224

Hardness as $\mathrm{CaCO} 3$

$(\mathrm{Ca}, \mathrm{Mg}) \quad 190$

Noncarbonate hardness as $\mathrm{CaCO} 3$

18

Percent sodium

Alkalinity as $\mathrm{CaCO} 3$

Specific conductance

(umhos $/ \mathrm{cm}$ at $25^{\circ} \mathrm{C}$ )

$\mathrm{pH}$ (units)

Temperature $\left({ }^{\circ} \mathrm{C}\right)$

Color (Pt-Co units)

Turbidity (NTU)

.00

ANALYSIS OF SELECTED TRACE ELEMENTS

(Total concentrations in micrograms per liter, except as indicated)

$\begin{array}{lr}\text { Arsenic (As) } & 1 \\ \text { Barium (Ba) } & 0 \\ \text { Cadmium (Cd) } & 0 \\ \text { Chromium (Cr) } & <10 \\ \text { Copper (Cu) } & 16 \\ \text { Iron (Fe) } & 70 \\ \text { Lead (Pb) } & 16\end{array}$

$\begin{array}{lc}\text { Manganese (Mn) } & 0 \\ \text { Mercury (Hg) } & <.5 \\ \text { Selenium (Se) } & 0 \\ \text { Silver ( } \mathrm{Ag}) & 0 \\ \text { Strontium, dissolved ( } \mathrm{Sr}) & 70 \\ \text { Zinc ( } \mathrm{n} \text { ) } & 180\end{array}$

ANALYSIS OF SELECTED PESTICIDE AND INDUSTRIAL COMP OUN S (Total concentrations in micrograms per liter)

$\begin{array}{llll}\text { Aldrin } & .00 & \text { Lindane } & .00 \\ \text { Chlordane } & .0 & \text { Methoxychlor } & .00 \\ \text { DDD } & .00 & \text { Mirex } & .00 \\ \text { DDE } & .00 & \text { PCB } & .0 \\ \text { DDT } & .00 & \text { PCN } & .00 \\ \text { Dieldrin } & .00 & \text { Silvex } & .00 \\ \text { Endrin } & .00 & \text { Toxaphene } & 0 \\ \text { Heptachlor } & .00 & 2,4-D & .00 \\ \text { Heptachlor epoxide } & .00 & 2,4,5-T & .00\end{array}$

ANALYSIS OF SELECTED GROSS RADIOACTIVITY

(Total activity concentrations in picocuries per liter)

Gross Alpha, as

uranium natural 2.9

Gross Beta, as

cesium-137
Gross Beta, as

strontium-90

2.4 
PUBLIC WATER SUPPLY: Wildwood

COUNTY: Sumter

COLLECTION DATE: 11-08-77

SAMPLING POINT (1) Treated water-285141082015501, tap outside pumphouse.

(2) Raw water-

ANALYSIS OF MAJOR CHEMICAL CONSTITUENTS AND PHYSICAL PROPERTIES (Dissolved concentrations in milligrams per liter, except as indicated)

10

Calcium ( $\mathrm{Ca}$ )

Magnesium ( $\mathrm{Mg})$

Sodium (Na)

Potassium ( $K$ )

Bicarbonate ( $\mathrm{HCO} 3) \quad 180$

Carbonate ( $\mathrm{CO} 3$ )

Sulfate (S04)

Chloride (C1)

Fluoride (F)

Nitrate (NO3-N), total

Nitrite (NO2-N),

total
(1)

60

(2)

2.6
5.4
.6
80
0
4.5
8.6
.1
1.1
.00

Dissolved solids

(residue at $180^{\circ} \mathrm{C}$ )

Hardness as $\mathrm{CaCO} 3$

$(\mathrm{Ca}, \mathrm{Mg})$

Noncarbonate hardness

as $\mathrm{CaCO} 3$

Percent sodium

Alkalinity as $\mathrm{CaCO} 3$

Specific conductance

(umhos $/ \mathrm{cm}$ at $25^{\circ} \mathrm{C}$ )

pH (units)

Temperature $\left({ }^{\circ} \mathrm{C}\right)$

Color (Pt-Co units)

Turbidity (NTU)
(1)

183

160

13

7

150

7.3

23.0

5

1

ANALYSIS OF SELECTED TRACE ELEMENTS

(Total concentrations in micrograms per liter, except as indicated)

$\begin{array}{lrlr}\text { Arsenic (As) } & 1 & \text { Manganese (Mn) } & 0 \\ \text { Barium (Ba) } & 0 & \text { Mercury (Hg) } & 15 \\ \text { Cadmium (Cd) } & 0 & \text { Selenium ( Se) } & 1 \\ \text { Chromium (Cr) } & <10 & \text { Silver (Ag) } & 1 \\ \text { Copper (Cu) } & 9 & \text { Strontium, dissolved ( } \mathrm{Sr} \text { ) } & 290 \\ \text { Iron (Fe) } & 50 & \text { Zinc (Zn) } & 60 \\ \text { Lead (Pb) } & 0 & & \end{array}$

ANALYSIS OF SELECTED PESTICIDE AND INDUSTRIAL COMPOUNDS

(Total concentrations in micrograms per liter)

$\begin{array}{llll}\text { Aldrin } & .00 & \text { Lindane } & .00 \\ \text { Chlordane } & .0 & \text { Methoxychlor } & .00 \\ \text { DDD } & .00 & \text { Mirex } & .00 \\ \text { DDE } & .00 & \text { PCB } & .0 \\ \text { DDT } & .00 & \text { PCN } & .00 \\ \text { Dieldrin } & .00 & \text { Silvex } & .00 \\ \text { Endrin } & .00 & \text { Toxaphene } & 0 \\ \text { Heptachlor } & .00 & 2,4-D & .00 \\ \text { Heptachlor epoxide } & .00 & 2,4,5-T & .00\end{array}$

ANALYSIS OF SELECTED GROSS RADIOACTIVITY

(Total activity concentrations in picocuries per liter)

Gross Alpha, as uranium natural $<2.2$

Gross Beta, as

cesium-137
Gross Beta, as

strontium -90

1.6 
PUBLIC WATER SUPPLY: Williston

COUNTY: LevY

COLLECTION DATE: . 11-03-77

SAMPLING POINT (1) Treated water-292309082272601, tap at water treatment plant.

(2) Raw water-

ANALYSIS OF MAJOR CHEMICAL CONSTITUENTS AND PHYSICAL PROPERTIES (Dissolved concentrations in milligrams per liter, except as indicated)

(1) (2)

$\begin{array}{lc}\text { Silica (SiO2) } & 6.3 \\ \text { Calcium (Ca) } & 88 \\ \text { Magnesium (Mg) } & 2.5 \\ \text { Sodium (Na) } & 3.7 \\ \text { Potassium (K) } & .4 \\ \text { Bicarbonate (HCO3) } & 280^{\circ} \\ \text { Carbonate (CO3) } & 0 \\ \text { Sulfate (SO4) } & 1.9 \\ \text { Chloride (C1) } & 6.5 \\ \text { Fluoride (F) } & .1 \\ \text { Nitrate (NO3-N), } & \\ \quad \text { total (NO2-N), } & 1.4 \\ \text { Nitrite (NO2-N) } \\ \quad \text { total }\end{array}$

(1)
Dissolved solids

$$
\text { (residue at } 180^{\circ} \mathrm{C} \text { ) } 254
$$

Hardness as $\mathrm{CaCO} 3$ ( $\mathrm{Ca}, \mathrm{Mg}$ )

Noncarbonate hardness as $\mathrm{CaCO}_{3}$

Percent sodium

Alkalinity as $\mathrm{CaCO} 3$

Specific conductance

(umhos $/ \mathrm{cm}$ at $25^{\circ} \mathrm{C}$ )

$\mathrm{pH}$ (units)

Temperature $\left({ }^{\circ} \mathrm{C}\right)$

Color (Pt-Co units)

Turbidity (NTU)
230

1

3

230

478

6.9

22.5

0

2

ANALYSIS OF SELECTED TRACE ELEMENTS

(Total concentrations in micrograms per liter, except as indicated)

$\begin{array}{lr}\text { Arsenic ( } \mathrm{As} \text { ) } & 0 \\ \text { Barium (Ba) } & 100 \\ \text { Cadmium (Cd) } & 0 \\ \text { Chromium ( } \mathrm{Cr} \text { ) } & 10 \\ \text { Copper (Cu) } & 4 \\ \text { Iron ( } \mathrm{Fe} \text { ) } & 30 \\ \text { Lead ( } \mathrm{Pb} \text { ) } & 6\end{array}$

Manganese $\left(\mathrm{Mn}_{\mathrm{n}}\right)$

Mercury ( $\mathrm{Hg}$ )

Selenium (Se)

Silver $\left(\mathrm{Ag}_{\mathrm{g}}\right)$

Strontium, dissolved (Sr) 110

zinc $(2 n)$
0

$<.5$

0

0

10

ANALYSIS OF SELECTED PESTICIDE AND INDUSTRIAL COMPOUNDS

(Total concentrations in micrograms per 1iter)

$\begin{array}{llll}\text { Aldrin } & .00 & \text { Lindane } & .00 \\ \text { Chlordane } & .0 & \text { Methoxychlor } & .00 \\ \text { DDD } & .00 & \text { Mirex } & - \\ \text { DDE } & .00 & \text { PCB } & .0 \\ \text { DDT } & .00 & \text { PCN } & .00 \\ \text { Dieldrin } & .00 & \text { Silvex } & .00 \\ \text { Endrin } & .00 & \text { Toxaphene } & 0 \\ \text { Heptachlor } & .00 & 2,4-D & .00 \\ \text { Heptachlor epoxide } & .00 & 2,4,5-T & .00\end{array}$

ANALYSIS OF SELECTED GROSS RADIOACTIVITY

(Total activity concentrations in picocuries per liter)

Gross Alpha, as

uranium natural

Gross Beta, as

cesium-137
Gross Beta, as

strontium -90 
PUBLIC WATER SUPPLY: Woodville

COUNTY: Leon COLLECTION DATE: $11-23-77$

SAMPLING POINT (1) Treated water-301853084145090, tap on west side of U.S. Post office building.

(2) Raw water-

ANALYSIS OF MAJOR CHEMICAL CONSTITUENTS AND PHYSICAL PROPERTIES (Dissolved concentrations in milligrams per liter, except as indicated)

\begin{tabular}{|c|c|}
\hline \multicolumn{2}{|l|}{$\begin{array}{l}\text { Silica ( } \mathrm{SiO2} \text { ) } \\
\text { Calcium ( } \mathrm{Cg}) \\
\left.\text { Magnesium ( } \mathrm{Mg}_{\mathrm{g}}\right) \\
\text { (agdium }(\mathrm{Na})\end{array}$} \\
\hline Potassium (K) & .4 \\
\hline Bicarbonate ( $\mathrm{HCO} 3$ ) & $270^{\circ}$ \\
\hline Carbonate ( $\mathrm{CO3})$ & \\
\hline Sulfate ( 504$)$ & $\begin{array}{l}7.4 \\
5.6\end{array}$ \\
\hline Fluoride (F) & .2 \\
\hline Nitrate $(\mathrm{NO} 3-\mathrm{N})$, & \\
\hline $\begin{array}{l}\text { total } \\
\text { Nitrite (NO2-N), }\end{array}$ & 1.0 \\
\hline - & \\
\hline
\end{tabular}
(2)

(1)

Dissolved solids

(reaidue at $180^{\circ} \mathrm{C}$ )

Hardness as $\mathrm{CaCO} 3$

$(\mathrm{C} 2, \mathrm{Mg})$

Noncarbonate hardness as $\mathrm{CaCO} 3$

Percent sodium

Alkalinity as $\mathrm{CaCO} 3$

Specific conductance

(umhos $/ \mathrm{cm}$ at $25^{\circ} \mathrm{C}$ )

$\mathrm{pH}$ (units)

Temperature $\left({ }^{\circ} \mathrm{C}\right)$

Color (Pt-Co units)

Turbidity (NTU)
233

240

18

220

450

7.4

19.5

4

1

ANALYSIS OF SELECTED TRACE ELEMENTS

(Total concentrations in micrograms per liter, except as indicated)

$\begin{array}{lrlc}\text { Arsenic (Ag) } & 2 & \text { Manganese (Mn) } & 10 \\ \text { Barium (Ba) } & 0 & \text { Mercury (Hg) } & 1.4 \\ \text { Cadmi (Cd) } & 5 & \text { Selenium (Se) } & 0 \\ \text { Chromium (Cr) } & <10 & \text { Silver (Ag) } & 0 \\ \text { Copper (Cu) } & 16 & \text { Strontium, dissolved (Sr) } & 90 \\ \text { Iron (Fe) } & 0 & \text { Zinc ( } \mathrm{n} \text { ) } & 390 \\ \text { Lead (Pb) } & 77 & & \end{array}$

ANALYSIS OF SELECTED PESTICIDE AND INDUSTRIAL COMPOUNDS

(Total concentrations in micrograms per liter)

$\begin{array}{llll}\text { Aldrin } & .00 & \text { Lindane } & .00 \\ \text { Chlordane } & .0 & \text { Methoxych10r } & .00 \\ \text { DDD } & .00 & \text { Mirex } & .00 \\ \text { DDE } & .00 & \text { PCB } & .0 \\ \text { DDT } & .00 & \text { PCN } & .00 \\ \text { Dieldrin } & .00 & \text { Silvex } & .00 \\ \text { Endrin } & .00 & \text { Toxaphene } & 0 \\ \text { Heptachlor } & .00 & 2,4-D & .00 \\ \text { Heptachlor epoxide } & .00 & 2,4,5-T & .00\end{array}$

ANALYSIS OF SELECTED GROSS RADIOACTIVITY

(Total activity concentrations in picocuries per liter)

Gross Alpha, as uranium natural $\quad 7.5$

Gross Beta, as

cesium-137
Gross Beta, as

strontium -90

3.8 
PUBLIC WATER SUPPLY: Zephyrhills

COUNTY: Pasco

COLLECTION DATE: 12-20-77

SAMPLING POINT (1) Treated water-281403082105309, tap outside fire station from elevated tank.

(2) Raw water-

ANALYSIS OF MAJOR CHEMICAL CONSTITUENTS AND PHYSICAL PROPERTIES

(Dissolved concentrations in milligrams per liter, except as indicated)

(1) (2)

$\begin{array}{lc}\text { Silica (SiO2) } & 9.4 \\ \text { Calcium (Ca) } & 48 \\ \text { Magnesium (Mg) } & 1.5 \\ \text { Sodium (Na) } & 3.8 \\ \text { Potassium (K) } & .3 \\ \text { Bicarbonate (HCO3) } & 110 \\ \text { Carbonate (CO3) } & 0 \\ \text { Sulfate (SO4) } & 3.0 \\ \text { Chloride (C1) } & 28 \\ \text { Fluoride (F) } & .1 \\ \text { Nitrate (NO3-N), } & \\ \quad \text { total } & 1.0 \\ \text { Nitrite (NO2-N), } & \\ \quad \text { total }\end{array}$

Dissolved solids

(1)

(residue at $180^{\circ} \mathrm{C}$ )

162

Hardness as $\mathrm{CaCO} 3$

( $\mathrm{Ca}, \mathrm{Mg}$ )

130

Noncarbonate hardness

as $\mathrm{CaCO} 3$

36

Percent sodium

Alkalinity as $\mathrm{CaCO} 3$

6

Specific conductance

(umhos $/ \mathrm{cm}$ at $25^{\circ} \mathrm{C}$ )

pH (units)

Temperature $\left({ }^{\circ} \mathrm{C}\right)$

Color (Pt-Co units)

Turbidity (NTU)

90

8.4
0
1

ANALYSIS OF SELECTED TRACE ELEMENTS

( Total concentrations in micrograms per liter, except as indicated)

$\begin{array}{lrlr}\text { Arsenic (As) } & 0 & \text { Manganese (Mn) } & 10 \\ \text { Barium (Ba) } & -- & \text { Mercury (Hg) } & 1.2 \\ \text { Cadmium (Cd) } & 0 & \text { Selenium (Se) } & 0 \\ \text { Chromium (Cr) } & 10 & \text { Silver (Ag) } & 2 \\ \text { Copper (Cu) } & 9 & \text { Strontium, dissolved (Sr) } & 90 \\ \text { Iron (Fe) } & 30 & \text { Zinc (Zn) } & 20 \\ \text { Lead (Pb) } & 10 & & \end{array}$

ANALYSIS OF SELECTED PESTICIDE AND INDUSTRIAL COMPOUNDS

(Total concentrations in micrograms per 1iter)

$\begin{array}{llll}\text { Aldrin } & .00 & \text { Lindane } & .00 \\ \text { Chlordane } & .0 & \text { Methoxych lor } & .00 \\ \text { DDD } & .00 & \text { Mirex } & .00 \\ \text { DDE } & .00 & \text { PCB } & .0 \\ \text { DDT } & .00 & \text { PCN } & .00 \\ \text { Dieldrin } & .00 & \text { Silvex } & .00 \\ \text { Endrin } & .00 & \text { Toxaphene } & 0 \\ \text { Heptachlor } & .00 & 2,4-D & .00 \\ \text { Heptachlor epoxide } & .00 & 2,4,5-\mathrm{T} & .00\end{array}$

ANALYSIS OF SELECTED GROSS RADIOACTIVITY

(Total activity concentrations in picocuries per liter)

Gross Alpha, as

uranium natural $<.9$

Gross Beta, as

cesium-137
Gross Beta, as

strontium $-90 \quad<.4$ 
PUBLIC WATER SUPPLY: Zolfo Springs COUNTY: Hardee COLLECTION DATE: 12-21-77

SAMPLING POINT (1) Treated water-272945081474409, tap on south side of pumphouse building.

(2) Raw water-

ANALYSIS OF MAJOR CHEMICAL CONSTITUENTS AND PHYSICAL PROPERTIES (Dissolved concentrations in milligrams per liter, except as indicated)

(1) (2) (1)

$\begin{array}{lc}\text { Silica (SiO2) } & 20 \\ \text { Calcium (Ca) } & 73 \\ \text { Magnesium (Mg) } & 34 \\ \text { Sodium (Na) } & 13 \\ \text { Potassium (K) } & 2.2 \\ \text { Bicarbonate (HCO3) } & 190 \\ \text { Carbonate (CO3) } & 0 \\ \text { Sulfate (SO4) } & \\ \text { Chloride (C1) } & 22 \\ \text { Fluoride (F) } & 1.0 \\ \text { Nitrate (NO3-N), } & \\ \quad \text { total (NO2-N), } & .03 \\ \text { Nitrite (NO2-N), } & \\ \quad \text { total } & .00\end{array}$

Dissolved solids

(residue at $180^{\circ} \mathrm{C}$ ) $\quad 477$

Hardness as $\mathrm{CaCO} 3$

$(\mathrm{Ca}, \mathrm{Mg}) \quad 360$

Noncarbonate hardness as $\mathrm{CaCO} 3$

Percent sodium

Alkalinity as $\mathrm{CaCO} 3$

Specific conductance

(umhos $/ \mathrm{cm}$ at $25^{\circ} \mathrm{C}$ )

$\mathrm{pH}$ (units)

Temperature $\left({ }^{\circ} \mathrm{C}\right)$

Color (Pt-Co units)

Turbidity (NTU)

200

8

160

ANALYSIS OF SELECTED TRACE ELEMENTS

(Total concentrations in micrograms per liter, except as indicated)

$\begin{array}{lc}\text { Manganese (Mn) } & 0 \\ \text { Mercury (Hg) } & <\cdot 5 \\ \text { Selenium ( Se) } & 0 \\ \text { Silver (Ag) } & 0 \\ \text { Strontium, dissolved ( } \mathrm{Sr}) 30000 \\ \text { Zinc ( } \mathrm{n} \text { ) } & 30\end{array}$

ANALYSIS OF SELECTED PESTICIDE AND INDUSTRIAL COMPOUNDS (Total concentrations in micrograms per liter)

$\begin{array}{llll}\text { Aldrin } & .00 & \text { Lindane } & .00 \\ \text { Chlordane } & .0 & \text { Methoxychlor } & .00 \\ \text { DDD } & .00 & \text { Mirex } & .00 \\ \text { DDE } & .00 & \text { PCB } & .0 \\ \text { DDT } & .00 & \text { PCN } & .00 \\ \text { Dieldrin } & .00 & \text { Silvex } & .00 \\ \text { Endrin } & .00 & \text { Toxaphene } & 0 \\ \text { Heptachlor } & .00 & 2,4-D & .00 \\ \text { Heptachlor epoxide } & .00 & 2,4,5-\mathrm{T} & .00\end{array}$

ANALYSIS OF SELECTED GROSS RADIOACTIVITY

(Total activity concentrations in picocuries per liter)

Gross Alpha, as uranium natural

Gross Beta, as

cesium-137
20

6.6
Gross Beta, as

strontium-90

5.8 



